University of Louisville

ThinkIR: The University of Louisville's Institutional Repository

Electronic Theses and Dissertations

$12-2007$

\title{
Perturbations in protein dynamics associated with ligand binding to the blood coagulation enzymes thrombin and Factor XIII.
}

Thomas Michael Sabo

University of Louisville

Follow this and additional works at: https://ir.library.louisville.edu/etd

\section{Recommended Citation}

Sabo, Thomas Michael, "Perturbations in protein dynamics associated with ligand binding to the blood coagulation enzymes thrombin and Factor XIII." (2007). Electronic Theses and Dissertations. Paper 1244. https://doi.org/10.18297/etd/1244

This Doctoral Dissertation is brought to you for free and open access by ThinkIR: The University of Louisville's Institutional Repository. It has been accepted for inclusion in Electronic Theses and Dissertations by an authorized administrator of ThinkIR: The University of Louisville's Institutional Repository. This title appears here courtesy of the author, who has retained all other copyrights. For more information, please contact thinkir@louisville.edu. 


\title{
PERTURBATIONS IN PROTEIN DYNAMICS ASSOCIATED WITH LIGAND BINDING TO THE BLOOD COAGULATION ENZYMES THROMBIN AND FACTOR XIII
}

\author{
By \\ Thomas Michael Sabo, II \\ B.S., University of Louisville, 2002 \\ M.S., University of Louisville, 2006

\begin{abstract}
A Dissertation
Submitted to the Faculty of the

Graduate School of the University of Louisville in Partial Fulfillment of the Requirements for the Degree of
\end{abstract} \\ Doctor of Philosophy \\ Department of Chemistry \\ University of Louisville \\ Louisville, Kentucky
}

December 2007 

Copyright 2007 by Thomas M. Sabo, II

All rights reserved 
PERTURBATIONS IN PROTEIN DYNAMICS ASSOCIATED WITH LIGAND BINDING TO THE BLOOD COAGULATION ENZYMES THROMBIN AND

FACTOR XIII

\author{
By \\ Thomas Michael Sabo, II \\ B.S., University of Louisville, 2002 \\ M.S., University of Louisville, 2006 \\ A Dissertation Approved on
}

September 4, 2007

by the following Dissertation Committee:

Muriel Maurer

Dissertation Director

John Arnez

George Pack

Michael Perlin

Cecilia Yappert 


\section{DEDICATION}

This dissertation is dedicated to my parents

Mr. Thomas M Sabo and Mrs. B.J. Reed

My dad lived with my brother and me in Bachelor's Paradise where he raised us when we were kids. I am forever grateful to you Dad for all the sacrifices you made for us and our well-being.

And to B.J. for putting up with the ungratefulness of my adolescence with only love and understanding, you are truly a saint.

\section{I love you both}

and

Mrs. Patricia M. Bunner and Mr. Hency Bunner

My mother brought me into this world on January 11, 1980. I am extremely thankful that we are a part of each other's lives and that I have been fortunate enough to live in Louisville with you for the last ten years.

And to Hency, who knows that I am truly the king of Chinese checkers

$$
\begin{gathered}
\text { I love you both } \\
\text { and }
\end{gathered}
$$

My partner in life, my pupskweeks, Karen Weeks

You are the most amazing woman on this planet

$$
\begin{gathered}
\text { I adore you } \\
\text { and }
\end{gathered}
$$

Our child, I have never been so anxious to meet a human being in my life

I love you both 


\section{AKNOWLEDGEMENTS}

The most influential figure in my academic career at the University of Louisville has been Professor Muriel Maurer. I cannot begin to express how grateful I am for your understanding, latitude, expertise, and friendship in my journey through graduate school, even when I happened to spill some invaluable peptide on the bench-top during a NMR prep. I also am indebted to my dissertation committee members, Professors John Arnez, George Pack, Mike Perlin, and Cecilia Yappert for their invaluable guidance and support.

I want to especially mention that Professor Perlin has been truly inspirational, primarily through his attitude towards life and towards those who are less fortunate. Keep fighting the good fight Professor Perlin, I aspire to be right there with you.

There are multitudes of people who have made an impact during my tenure as a graduate student. I list them in no particular order. Professor Elizabeth Komives from the University of California, San Diego has been instrumental in passing on the secrets of hydrogen deuterium exchange coupled with MALDI-TOF MS. I greatly appreciate her knowledge and advice during the course of this research. The thrombin projects would never have been conducted if not for a wonderful collaboration between Professor Maurer and Professor David Farrell at the Oregon Health Sciences University in Portland, OR. Thanks Professor Farrell for the initial amount of $\gamma^{\prime}$ peptide that I spilled, this led Professor Maurer to have some peptide synthesized.

Professor Christine Rich is an extremely gifted teacher and communicator. Thank you for allowing me to be your TA in organic chemistry. I learned more about organic 
chemistry as your TA than as a student. I would like to thank Professor Andrew Lane and Dr. Sam Arumugam for their guidance and assistance during the course of the 800 MHz experiments. Also, Dr. Neal Stolowich for his invaluable help in working with the 500 MHz NMR. Professor J. Brad Chaires, Professor Bill Dean, and Dr. Nichola Garbett for their expertise in ITC and AUC, as well as allowing me to work in the Biophysics laboratory at the Brown Cancer Center. I would like to mention that Nichola has been extraordinarily generous with her time and advice. Thank you Nichola. I am extremely grateful to Professor Enrico Di Cera and Dr. Augustus Pineda at Washington University in St. Louis, MS for releasing the coordinates for the X-ray structure of the $\gamma^{\prime}$ peptide binding to IIa before being released.

I would like to acknowledge all my colleagues in the Maurer lab past and present: Dr. Toni Trumbo-Bell, Dr. Brian Turner, Dr. Giulia Isetti, Dr. David Cleary, P. Brad Brasher, Eric Saunders, Prakash Doiphode, Madhavi Jadhav, Marina Malovichko and Cory Lucas.

I would like to give love to all my amazingly talented and uniquely gifted friends that I have been lucky to know since moving from Pennsylvania to Louisville 10 years ago. Tony Lombardi was instrumental in putting the copies of the dissertation together. Thank you Tony for introducing me to mindless work. See you at Alex's place.

I miss my buddies Aaron Todovich and Tim Cushing. If only....

Finally, to the love of my life Karen Weeks. Though life has made a few decisions without consulting us, I have never felt so comfortable and at peace with another human being in my life. We truly balance each other and I look forward to all our future experiences together. I love you! 


\section{ABSTRACT \\ PERTURBATIONS IN PROTEIN DYNAMICS ASSOCIATED WITH LIGAND BINDING TO THE BLOOD COAGULATION ENZYMES THROMBIN AND FACTOR XIII}

Thomas M Sabo, II

December 13, 2007

In blood coagulation, the serine protease thrombin is a multifaceted enzyme that interacts with multiple proteins. Thrombin utilizes two anion binding exosites (ABE-I and II) to supplement binding to fibrinogen and to the platelet receptor GpIb $\alpha$, two proteins instrumental in clot formation. Approximately $7 \%$ to $15 \%$ of the fibrinogen $\gamma$ chain exists as the highly anionic $\gamma^{\prime}$ variant $\left({ }^{410}\right.$ PEHPAETEY $_{S} D S L Y_{S}$ PEDDL $\left.^{427}\right)$, which has been shown to bind ABE-II. GpIbo possesses a similar anionic stretch of residues $\left({ }^{269}\right.$ DEGDTDLY $_{S}$ DY $_{S} Y_{S}$ PEEDTEG $\left.^{286}\right)$; however, the exact destination of thrombin binding is more ambiguous. $1 \mathrm{D}$ and 2D solution NMR have been employed to characterize the structural features of the bound $\gamma^{\prime}$ and GpIb $\alpha$ peptides. The results indicate that the $\gamma^{\prime}$ residues $\mathrm{A}^{414}-\mathrm{L}^{427}$ make significant contact with the enzyme, a turn is present between residues $\mathrm{Y}_{\mathrm{S}}{ }^{422}-\mathrm{D}^{425}$, and there is a hydrophobic cluster involving $\mathrm{Y}_{\mathrm{S}}{ }^{418}$ $\mathrm{Y}_{\mathrm{S}}{ }^{422}$. For the GpIbo peptide, NMR results suggest the peptide exists in an extended conformation with residues $\mathrm{D}^{274}-\mathrm{E}^{285}$ contributing to binding. Hydrogen deuterium exchange (HDX) coupled with MALDI-TOF MS indicate that at a 20:1 peptide-enzyme ratio both peptides are interacting with residues within ABE-II fragments. Both peptides 
affect the dynamics of HDX for regions not associated with ABE-II including ABE-I, the autolysis loop, and the A-chain. Curiously, at 40:1 GpIb $\alpha$ peptide to thrombin, the GpIb $\alpha$ peptide appears to interact with ABE-I, with unique allosteric consequences. Thrombin also activates FXIII, and the resultant transglutaminase covalently crosslinks fibrin through the formation of isopeptide bonds. Studies were performed to examine the effects of activation and inhibition on the conformational dynamics of FXIII using MALDI-TOF MS. A peptide-based FXIIIa inhibitor has been developed with the glutamine isostere 6-diazo-5-oxo-norleucine (DON). The K9 DON peptide blocks chemical modification of $\mathrm{C}^{409}$ within the catalytic core and promotes significant HDX protection for the $\beta$ barrel 1 fragment 526-546. These results suggest that inhibition of FXIIIa leads to local and long-range effects on protein dynamics. Finally, thrombin activation of FXIII appears to influence regions near the activation peptide, and $\mathrm{Ca}^{2+}$ activated FXIII presents more evidence for the existence of additional $\mathrm{Ca}^{2+}$ binding sites. Ultimately, these studies further detail the dynamic nature of thrombin and FXIII. 
TABLE OF CONTENTS

PAGE

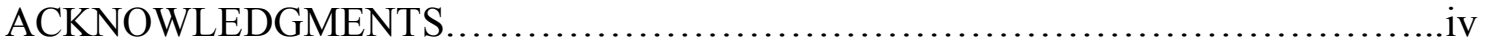

ABSTRACT ..................................................................

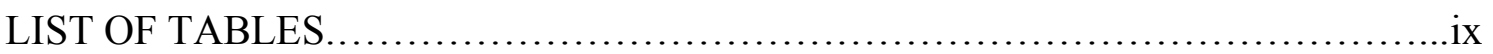

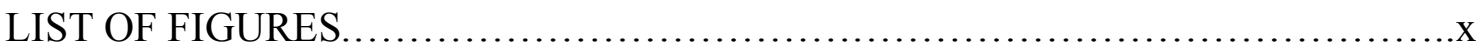

CHAPTER

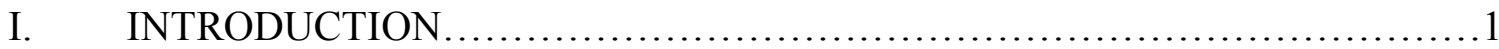

II. NUCLEAR MAGNETIC RESONANCE TECHNIQUES:

1D LINE BROADENING AND 2D TRANSFERRED NOESY .................29

III. MATRIX ASSISTED LASER DESORPTION IONIZATION

TIME-OF-FLIGHT MASS SPECTROMETRY

AND HYDROGEN DEUTERIUM EXCHANGE.

IV. CONFORMATIONAL ANALYSIS OF $\gamma^{\prime}$ PEPTIDE (410-427)

INTERACTIONS WITH THROMBIN ANION BINDING EXOSITE-II........56

V. STUDYING GPIb $\alpha$ (269-286) PEPTIDE INTERACTIONS

WITH THE THROMBIN ANION BINDING EXOSITES.

VI. PERTURBATIONS IN FACTOR XIII RESULTING FROM

ACTIVATION AND INHIBITION EXAMINED BY SOLUTION

BASED METHODS AND DETECTED BY MALDI-TOF MS

VII. RESEARCH SUMMARY AND FUTURE DIRECTIONS ..................150

REFERENCES......................................................... 169

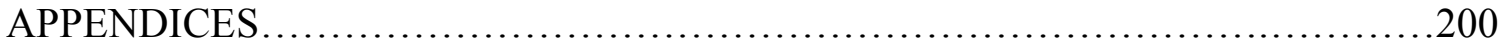

CURRICULUM VITAE..................................................216 


\section{LIST OF TABLES}

TABLE

PAGE

1. Sequences of Selected ABE-II Binding Sites.................................13

2. Common Matrices Used with MALDI-TOF MS .............................41

3. $\gamma^{\prime}$ Peptides Used in IIa Binding Studies........................................59

4. Deuterium Incorporation for IIa, PPACK-IIa, $\gamma^{\prime}$-IIa, and $\gamma^{\prime}$ PPACK-IIa

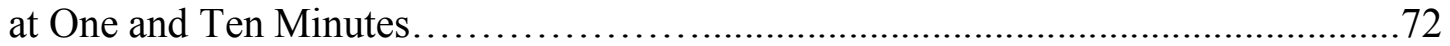

5. Changes in \% Deuteration for IIa, PPACK-IIa, $\gamma^{\prime}$-IIa, and $\gamma^{\prime}$ PPACK-IIa at One and Ten Minutes Relative to Free IIa................................ 76

6. GpIb $\alpha$ Peptides Used in IIa Binding Studies.................................90

7. Deuterium Incorporation for IIa, GpIb $\alpha$-IIa, and $\gamma^{\prime}$-IIa at One and Ten Minutes.......

8. Changes in \% Deuteration for IIa, GpIb $\alpha$-IIa, and $\gamma^{\prime}$-IIa at One and Ten Minutes Relative to Free IIa .100

9. Analytical Ultracentrifugation Data. 105

10. Changes in \% Deuteration for Factor XIII-Inhibitor Complexes at One and Ten Minutes Relative to Unactivated Factor XIII.

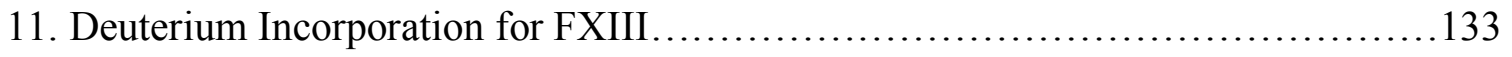

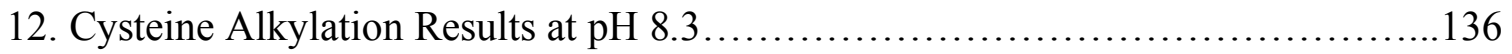

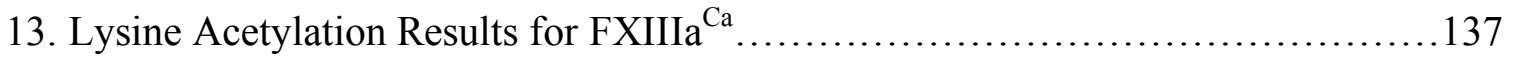

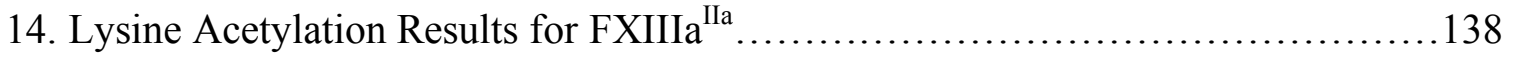




\section{LIST OF FIGURES}

FIGURE

PAGE

1. Classical view of blood coagulation........................................

2. Blood coagulation according to Monroe and Hoffman...........................

3. Schematic of the conversion of prothrombin to thrombin.......................... 8

4. Structure of the serine protease thrombin................................. 10

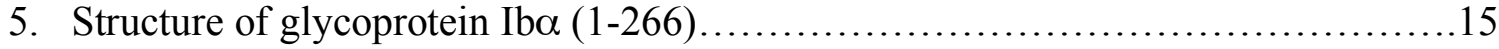

6. Schematic diagram depicting the structure of fibrinogen and clot formation..........18

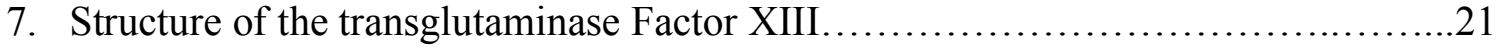

8. Proposed catalytic mechanism of transglutaminases............................22

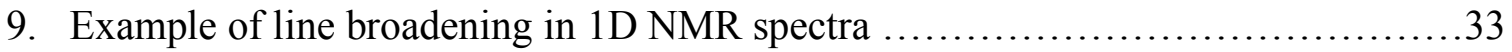

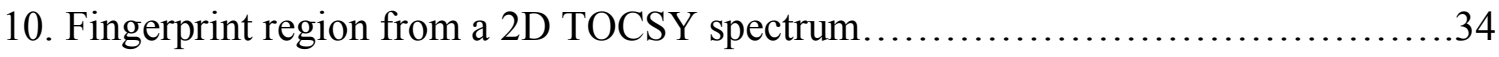

11. 2D transferred NOESY spectrum......................................... 35

12. Summary of the 1D line broadening and 2D transferred NOESY techniques........36

13. Schematic of a MALDI-TOF mass spectrometer...........................40

14. NEM modification of cysteine as detected by MALDI-TOF MS ..................45

15. Acetic Anhydride modification of lysine as detected by MALDI-TOF MS.........46

16. Pathways of protein backbone amide proton exposure $\ldots \ldots \ldots \ldots \ldots \ldots \ldots \ldots \ldots \ldots . . .48$

17. Protocol for the hydrogen deuterium exchange experiment.....................49

18. Isotopic envelope for a peptide after 0,1 , and 10 minutes of deuteration............53 


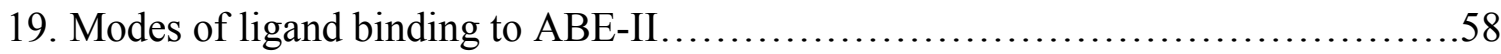

20. Line broadening for the $\gamma^{\prime}\left(Y_{P}^{418} Y_{P}^{422}\right)$ peptide in the presence of thrombin..........64

21. Line broadening for the $\gamma^{\prime}\left(Y_{P}{ }^{422}\right)$ peptide in the presence of thrombin...............65

22. Line broadening for the $\gamma^{\prime}\left(\mathrm{Y}_{\mathrm{P}}{ }^{418}\right)$ peptide in the presence of thrombin...............66

23. Representative spectra of key $\gamma^{\prime}$ peptide NOEs in the presence of thrombin..........67

24. ${ }^{31} \mathrm{P} 1 \mathrm{D}$ NMR spectra for the $\gamma^{\prime}\left(\mathrm{Y}_{\mathrm{P}}{ }^{418} \mathrm{Y}_{\mathrm{P}}{ }^{422}\right)$ peptide in the presence of thrombin.....69

25. Sequence coverage from the peptic digest of bovine thrombin.......................70

26. Graphs of deuterium incorporation for IIa, PPACK-IIa, $\gamma^{\prime}$-IIa, and $\gamma^{\prime}$ PPACK-IIa....71

27. Mass spectra of IIa residues $85-94$ after one and ten minutes of HDX................73

28. Mass spectra of IIa residues 65-84 and 212-227 after one minute of HDX............74

29. Crystallographic views of the $\gamma^{\prime}$ peptide bound to thrombin (I) .....................8 80

30. Crystallographic views of the $\gamma^{\prime}$ peptide bound to thrombin (II) ...................82

31. Correlating HDX results with X-ray structure of PPACK-IIa........................84

32. Two views of GpIb $\alpha$ binding to thrombin...................................... 88

33. Line broadening spectra for the GpIb $\alpha$ peptides in the presence of thrombin........94

34. Representative spectra of GpIb $\alpha$ peptide NOEs in the presence of thrombin.........95

35. Graphs of deuterium incorporation for IIa, GpIb $\alpha$-IIa, and $\gamma^{\prime}$-IIa at 20:1 ...........97

36. Mass spectra of residues 85-99 after one and ten minutes of HDX..................99

37. Graphs of deuterium incorporation for IIa, GpIb $\alpha$-IIa, and $\gamma^{\prime}$-IIa at 40:1 .........102

38. Mass spectra of residues 65-84 after one minute of HDX..........................104

39. Sedimentation velocity AUC of GpIb $\alpha$ peptide $(14 \mu \mathrm{M})$ and IIa $(7 \mu \mathrm{M}) \ldots \ldots \ldots \ldots . . .106$

40. Sedimentation velocity AUC of $\gamma^{\prime}$ peptide $(4.7 \mu \mathrm{M})$ and IIa $(4.7 \mu \mathrm{M}) \ldots \ldots \ldots \ldots \ldots . . .107$

41. Crystallographic views of GpIba bound to thrombin (I).........................110 


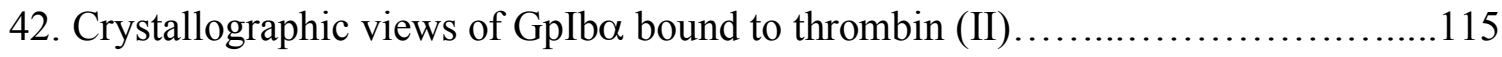

43. Summary of previous results reported on Factor XIII dynamics..................120

44. K9 DON inhibition of FXIIIa.......................................... 122

45. Mass spectrum of the K9 DON modified $C^{314}$ from a tryptic digest................124

46. Sequence coverage map of FXIII..................................... 127

47. TGase assay and FXIIIa inhibition by K9 DON............................ 130

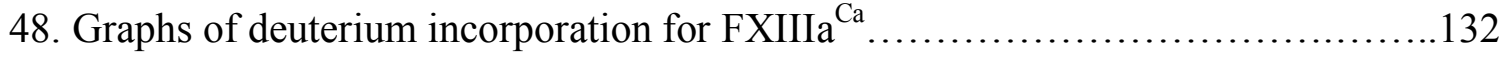

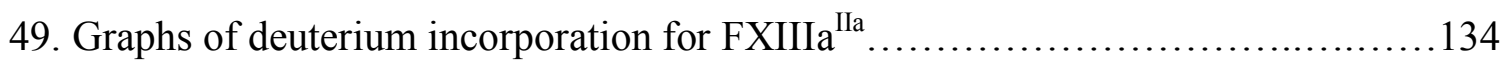

50. Mass spectra of residues 526-546 after ten minutes of HDX $\ldots \ldots \ldots \ldots \ldots \ldots \ldots \ldots \ldots \ldots$

51. Graph of limited chymotryptic proteolysis of FXIII...........................139

52. Correlating results with X-ray structure of Factor XIII (I) .....................141

53. Correlating results with X-ray structure of Factor XIII (II) ...................... 145

54. Isothermal calorimetry of the GpIb $\alpha$ peptide and thrombin.....................159 


\section{CHAPTER I}

\section{INTRODUCTION}

\section{Overview of Blood Coagulation}

One of the most precious physiological tissues exists in the fluid state. Blood travels throughout the body and is maintained in the vasculature. A multifaceted substance, blood serves as a transport system for cellular components, proteins, nutrients, hormones, and waste. Blood shuttles red blood cells to and from the lungs, where hemoglobin loads oxygen for aerobic metabolism in cells and deposits the waste product carbon dioxide. In the case of infection, blood transports white blood cells to the site of the foreign invasion. The white blood cells then attack the threat. Thus, the vitality of life clearly depends on maintaining blood in an unimpeded fluid state and in an enclosed network of highways (the blood vessels) that connect even the most distant regions of the $\operatorname{organism}(1,2)$.

When a vascular breach or damaged tissue is present, circulating platelets and proteins are ready to quickly seal the leak in a process termed coagulation. Unchecked clotting, however, could potentially lead to a life threatening obstruction of blood flow, termed thrombosis. Through evolutionary processes, life has developed a tightly regulated system of clot formation and degradation, involving cells, proteins, sugars, electrolytes and other molecules. Further elucidation into the mechanisms of blood coagulation will eventually open new avenues for treatment of hypertension, heart disease, and hemophilia $(1,2)$. 
The classical view of blood coagulation as a cascade of events separated into two pathways that converge with the activation of Factor X (FX) is presented in Figure 1. Tissue damage leads to activation of the extrinsic, or tissue factor (TF), pathway $(3,4)$. Physiologically, this pathway is the most prompt route for wound healing. The components of the intrinsic, or contact factor, pathway all reside within the circulating

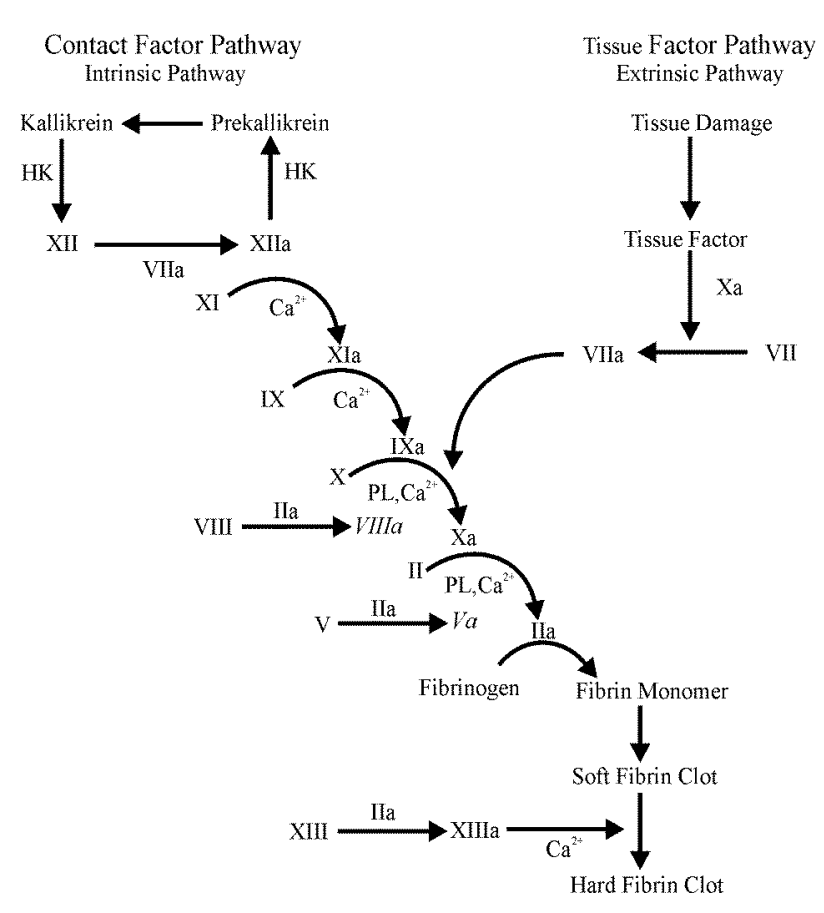

Figure 1: Classical view of blood coagulation. The cascade is separated into the intrinsic and extrinsic pathways. Adapted from a poster by Enzyme Research Laboratories, South Bend, IN.

plasma (5). Initiation of this branch of coagulation requires more time for full activation. A few perplexing issues exist regarding this model of blood clotting. The main quandary deals with the absence of any blood clotting deficiencies in patients with low levels of FXII (6). However, FVIII and FIX from the intrinsic pathway are absolutely essential for coagulation, since patients lacking sufficient levels of these proteins in the plasma suffer from hemophilia (7). Finally, a dearth in FVII from the extrinsic pathway also produces severe clotting disorders illustrating that the intrinsic pathway alone cannot support effective wound healing (8). These observations exemplify the interrelatedness of both pathways and suggest that coagulation should not be thought of as two separate conduits but a more integrated process without clear pathway demarcations. 


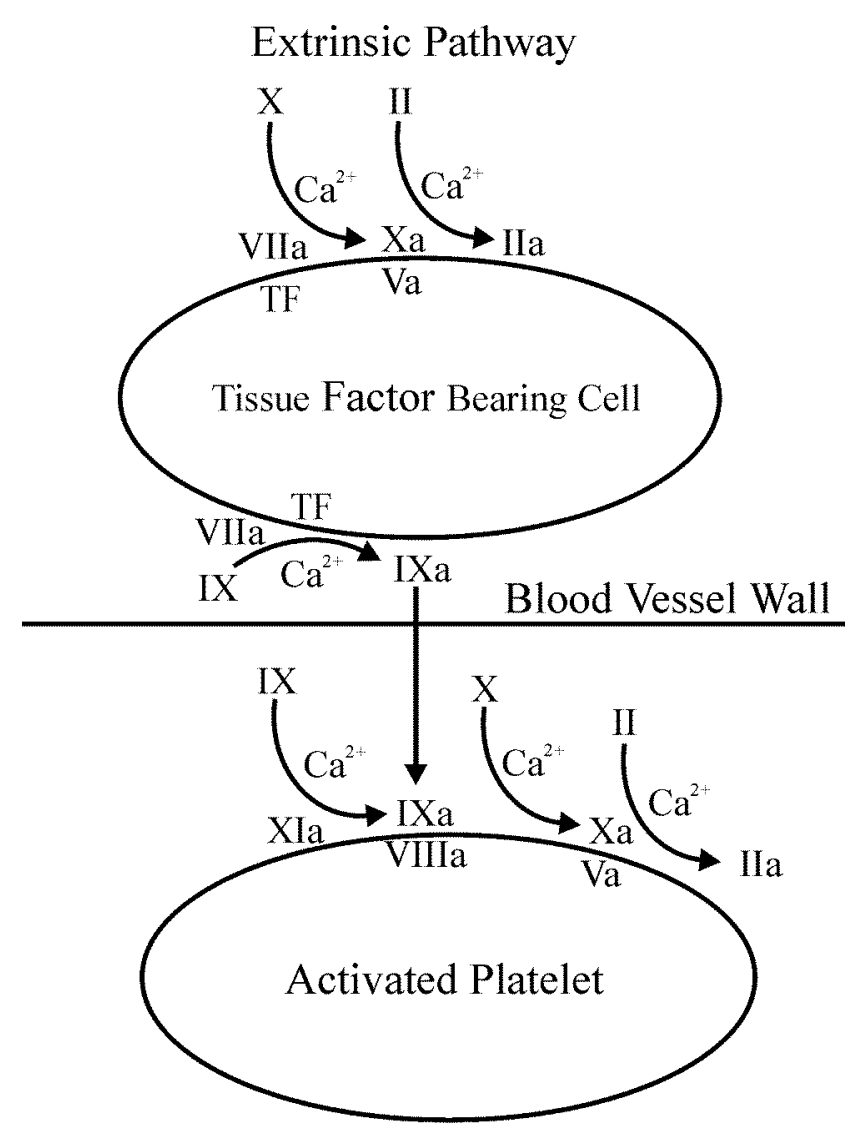

Intrinsic Pathway

Figure 2: Blood coagulation according to Monroe and Hoffman (9-11). The classical view of blood coagulation separates the process into two distinct pathways converging on the activation of FX. The cellbased model of blood coagulation emphasizes the interrelatedness of both pathways as described in the text. In this figure, procoagulation events are depicted in relation to the cell surface on which they occur.
Recent proposals from Hoffman

and Monroe attempting to rationalize the clinical observations contradicting the view of coagulation as two pathways have developed the cell-based model of haemostasis ( 9 , 10). In this scheme, coagulation is separated into three stages, initiation, amplification, and propagation (11). Figure 2 presents the extrinsic and intrinsic pathways in terms of the cell-based model of coagulation. Regardless of whether this model or the classical views of the coagulation cascade are accurate representations of blood clotting, the complexity of thrombus formation should be readily apparent with a cursory glance at

Figures 1 and 2. In an effort to present blood coagulation with a fresh perspective, the process of clot formation, regulation, and, ultimately, lysis will be discussed in terms of the cell-based model of Hoffman and Monroe.

Proper synthesis of prothrombin, FVII, FIX, and FX calls for vitamin K which is a cofactor in the conversion of glutamic acid to $\gamma$-carboxyglutamic acid (Gla) (12). These 
proteins' Gla domains reside at the N-termini and provide the site for interaction with the surface of cell membranes involved with blood coagulation. Binding to the phospholipid membranes has the additional requirement of calcium. $\mathrm{Ca}^{2+}$ serves to stabilize the Gla domains of these vitamin K-dependent proteins and to bridge the negatively charged phospholipid membrane to the negatively charged Gla domains of the proenzymes (13). Without the Gla domains, successful initiation of blood coagulation is significantly hindered. In the following discussion of coagulation, productive activation and/or function of prothrombin, FVII, FIX, and FX all require $\mathrm{Ca}^{2+}$ and a cell surface.

The initiation phase of clotting is characterized by the preparation of extravascular cells for an appropriate response to tissue damage. TF bearing cells typically exist outside the vasculature, i.e. subendothelial tissue. TF is a transmembrane protein formally known as FIII. It should be noted that TF also resides in the blood, although the physiological significance of the circulating TF has yet to be determined (3). Even in the absence of vascular injury, several clotting factors are able to diffuse into the extravascular space. Some of the TF forms a complex with the serine protease FVIIa that can successfully activate FIX and FX in small quantities (14).

The low levels of FXa are able to activate FV and FVII present on TF bearing cells (15). The resulting prothrombinase complex $(\mathrm{FVa} / \mathrm{FXa})$ can convert prothrombin to thrombin (IIa) (3). Like FXa, IIa also can activate FVII (16). These events explain the basal level of active coagulation factors that are present in normal individuals. Fortunately for proper haemostatic balance, once FXa diffuses from the TF bearing cell and returns to the blood stream, antithrombin and the TF pathway inhibitor (TFPI) inactivate the protease (17). An explanation for the lack of full blown clotting resides in 
the sizes of the FVIII/von Willebrand factor (vWF) complex and platelets which effectively minimizes the amount of diffusion that can occur, maintaining these coagulation factors in the vasculature (18).

Amplification of clotting commences when damage to the endothelium allows platelets, which are circulating in the blood, to contact the exposed tissue. Platelets adhere to collagen and other extracellular matrix components that are available at the site of tissue damage (19). Adhesion of platelets to the damaged tissue only partially activates the blood cells. IIa activated by TF bearing cells during the initiation phase can now cleave the protease activated receptors (PARs) leading to the activation of platelets (20). FVIII/vWF binds to the platelet surface and activation of FVIII by IIa releases vWF $(18,21)$. IIa also activates FV (22) and FXI (23) located on the platelet membrane. The ability of IIa to activate FXI on the activated platelet surface alleviates the requirement for FXII, high molecular weight kininogen (HK), and prekallikrein (PK) for FXI activation (10). This point is highlighted because in the classical model of coagulation, FXII, HK, and PK initiate intrinsic coagulation through activation of FXI (24). As evident in the amplification phase, the small amount of available IIa primes the system for full-blown thrombin activation and clot formation (9-11).

In the final phase of coagulation, a burst of IIa generation is evident as well as a propagation of procoagulant activities through positive feedback mechanisms. The low levels of FIXa from the initiation phase forms the tenase complex with FVIIIa on the platelet surface (25-27). FXIa bound to the platelet membrane generates additional activated FIX $(28,29)$. Since FXa produced on TF bearing cells is unable to effectively travel to the platelets, the tenase complex activates FX on the platelet surface (9-11). 
FXa associates with FVa forming the prothrombinase complex, which catalyzes the conversion of prothrombin to IIa (3). Monroe and Hoffman emphasize that the platelet surface is the only cell adequately designed to support tenase and prothrombinase complex formation concurrently (9-11). In hemophilia, deficient quantities of FVIII or FIX on the platelet surface severely limit that amount of thrombin activated at the site of injury $(7,30,31)$. Though TF bearing cells can activate thrombin, this pathway is insufficient in alleviating the loss of the tenase complex formation on platelets (9-11).

At the nexus of procoagulation and anticoagulation resides the most important coagulation protein thrombin (32-34). The procoagulant activities of thrombin are multifaceted and numerous. Thrombin converts fibrinogen to fibrin which associates non-covalently to form the soft clot (35). Stabilization of the growing fibrin clot is provided by IIa-activated FXIII, a transglutaminase that forms covalent linkages between neighboring fibrin molecules $(36,37)$, and thrombin-activatable fibrinolysis inhibitor (TAFI), a metalloprotein that protects the growing clot from degradation (38). In addition, thrombin participates in procoagulant positive feedback by activating FV (22), FVIII (18), and FXI (39). Finally as mentioned earlier, several platelet receptors are cleaved by this serine protease leading to the activation of platelets (20).

Obviously, unchecked coagulation would be fatal due to the cessation of blood circulation. The activation of the anticoagulant protein C (PC) by IIa is accelerated when IIa encounters the glycoprotein thrombomodulin on the surface of endothelial cells (40). Activated PC turns off IIa production by cleaving FVa and FVIIIa disrupting productive tenase and prothrombinase complex formation (41). Inhibition of coagulation proteases by the serpin antithrombin is another method for shutting down coagulation (42). The 
glycosaminoglycan (GAG) heparin enhances successful inhibition of these proteases with antithrombin, with the acceleration of thrombin inhibition augmented up to 1000-fold (43). Heparin cofactor II is another serpin that specifically targets IIa, with inactivation improved in the presence of GAGs (44).

Clots exist as temporary solutions to repairing wounds and eventually degradation occurs by the fibrinolytic system. Tissue-type plasminogen activator (tPA) and plasminogen are the major players in this process. Activation of plasminogen to plasmin results from ternary complex formation between tPA, plasminogen, and fibrin (45). Active plasmin proceeds to hydrolyze fibrin at specific sites within the clot, ultimately leading to the dissolution of the clot (35). To prevent premature clot lysis, several proteins inhibit the protease plasmin. $\alpha_{2}$-antiplasmin not only acts as an inhibitor of plasmin, the protein also blocks the binding of plasminogen to the fibrin clot preventing activation of the fibrinolytic protease (46). FXIIIa acts to covalently crosslink $\alpha_{2}$ antiplasmin into the growing clot another preventive measure against unwarranted fibrinolysis (36). The serine protease plasminogen activator inhibitor type 1, when bound to fibrin, inhibits tPA and thus plasminogen activation (47). As mentioned previously, TAFI activated by the thrombin/thrombomodulin complex serves as another mechanism for the prevention of clot lysis (38).

Only a brief summary has been presented of the processes that encompass blood coagulation. The majority of the projects described in this dissertation focus on the proteins located at the end of coagulation, specifically thrombin and FXIII. A more detailed analysis of the relevant features associated with these proteins will be discussed in the following sections. With regard to thrombin, the research undertaken has 
examined peptide interactions at anion binding exosite II of this serine protease. These peptides are derived from sequences that exist in fibrinogen and GpIba. Therefore, a more in depth view of these proteins will be presented in context to interactions with IIa.

\section{The Most Important Coagulation Protease: Thrombin}

Circulating in the blood stream and awaiting activation by the prothrombinase complex is the 579-residue zymogen prothrombin $(48,49)$. Prothrombin is a glycoprotein that is synthesized in the liver and circulates in the blood stream at $\sim 1.2$ $\mu \mathrm{M}$. The zymogen possesses several domains including an $\mathrm{N}$-terminal Gla domain, two kringle domains, and a proteolytic domain that is dormant until activation. Within the Gla domain, 10 glutamic acids residues are converted to $\gamma$ carboxyglutamic acid through vitamin Kdependent mechanisms. Kringle domains are so named due to their resemblance to the Danish pastry. These structures form large loops that are stabilized by three

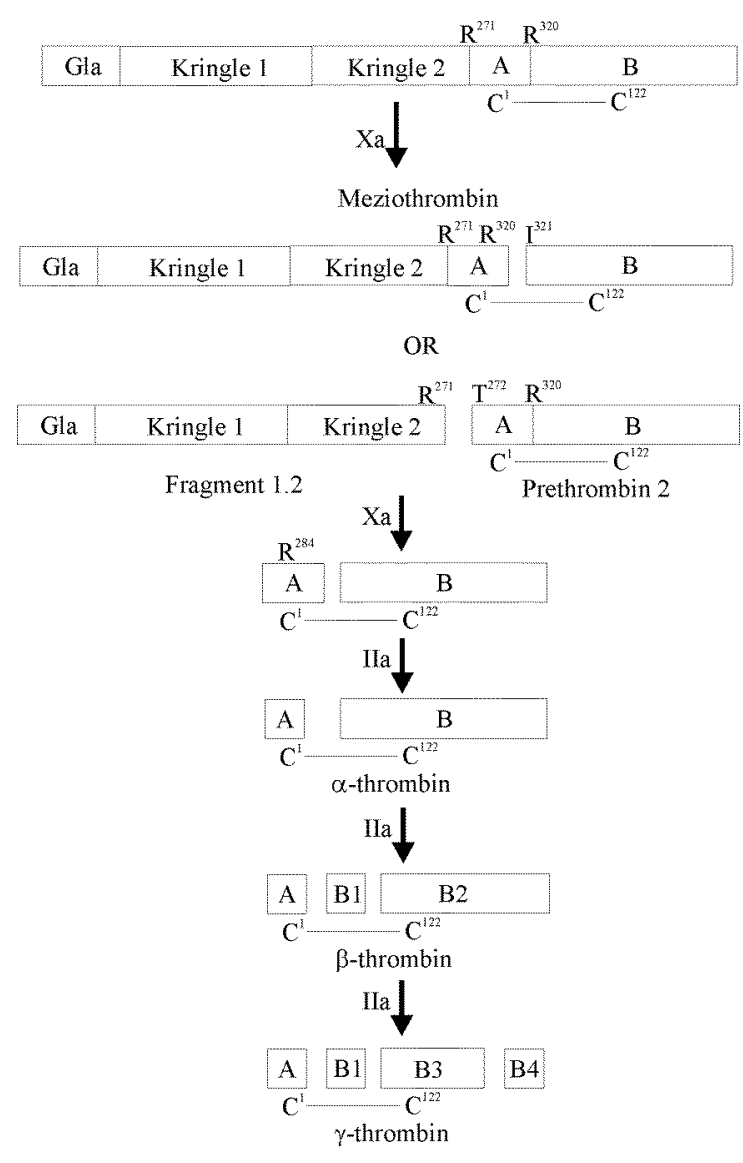

Figure 3: Schematic of the conversion of prothrombin to thrombin. Depicted are the various IIa precursors and autolytic products. disulfide bonds.

Conversion of prothrombin to $\alpha$-thrombin (IIa) proceeds through several proteolytic steps (see Figure 3) catalyzed by the prothrombinase complex (FXa/FVa) in the presence 
of $\mathrm{Ca}^{2+}$ on either the platelet surface or on a TF bearing cell (reviewed in 49). FXa hydrolyzes two peptide bonds, though the order of cleavage has yet to be established. Proteolysis at $\mathrm{R}^{320}-\mathrm{I}^{321}$ results in meziothrombin with fragment 1.2 and the A-chain associated to IIa's proteolytic domain through a disulfide bond $\left(\mathrm{C}^{1}-\mathrm{C}^{122}\right.$ in chymotrypsin numbering, see below). Prethrombin 2 is formed when FXa first hydrolyzes $\mathrm{R}^{271}-\mathrm{T}^{272}$ leading to dissociation of fragment 1.2. When FXa cleaves the second bond, $\alpha$-IIa is produced with the A-chain associated to the B-chain through a disulfide linkage. Once $\alpha$-IIa possesses catalytic activity, autolysis of the A-chain residues $\mathrm{R}^{284}-\mathrm{T}^{285}$ yields mature $\alpha$-IIa. $\alpha$-IIa is $37.5 \mathrm{kDa}$ with six $\alpha$-helices, two $\beta$-sheets, and four disulfide bonds (50). One of the disulfide linkages is between the A and B-chains, while the remaining disulfide bonds are all within the B-chain.

For ease of comparison to other members of the serine protease family, $\alpha$-IIa residue numbering is referenced relative to chymotrypsin (51). When describing residues in loops that are unique to IIa or just single amino acid insertions that are not present in chymotrypsin, a letter follows the number of the last common residue. For example, two important loops are specific to IIa and begin from the last common residue shared between the two proteases. The $\beta$ insertion loop begins at 60 and continues with $60 \mathrm{~A}$, 60B, etc. The autolysis loop starts from 149, and continues from 149A, 149B, and so on. The N-terminus of IIa's A-chain possesses eight additional residues when compared to chymotrypsin. Thus, A-chain numbering begins at $1 \mathrm{H}$ and continues $1 \mathrm{G}, 1 \mathrm{~F}$, etc. It should be noted that bovine $\alpha$-IIa lacks an autolytic site within the A-chain. Therefore, bovine $\alpha$-IIa possesses an additional 22 residues at the $\mathrm{N}$-terminus when compared to chymotrypsin and 13 residues when compared to human $\alpha$-IIa. 


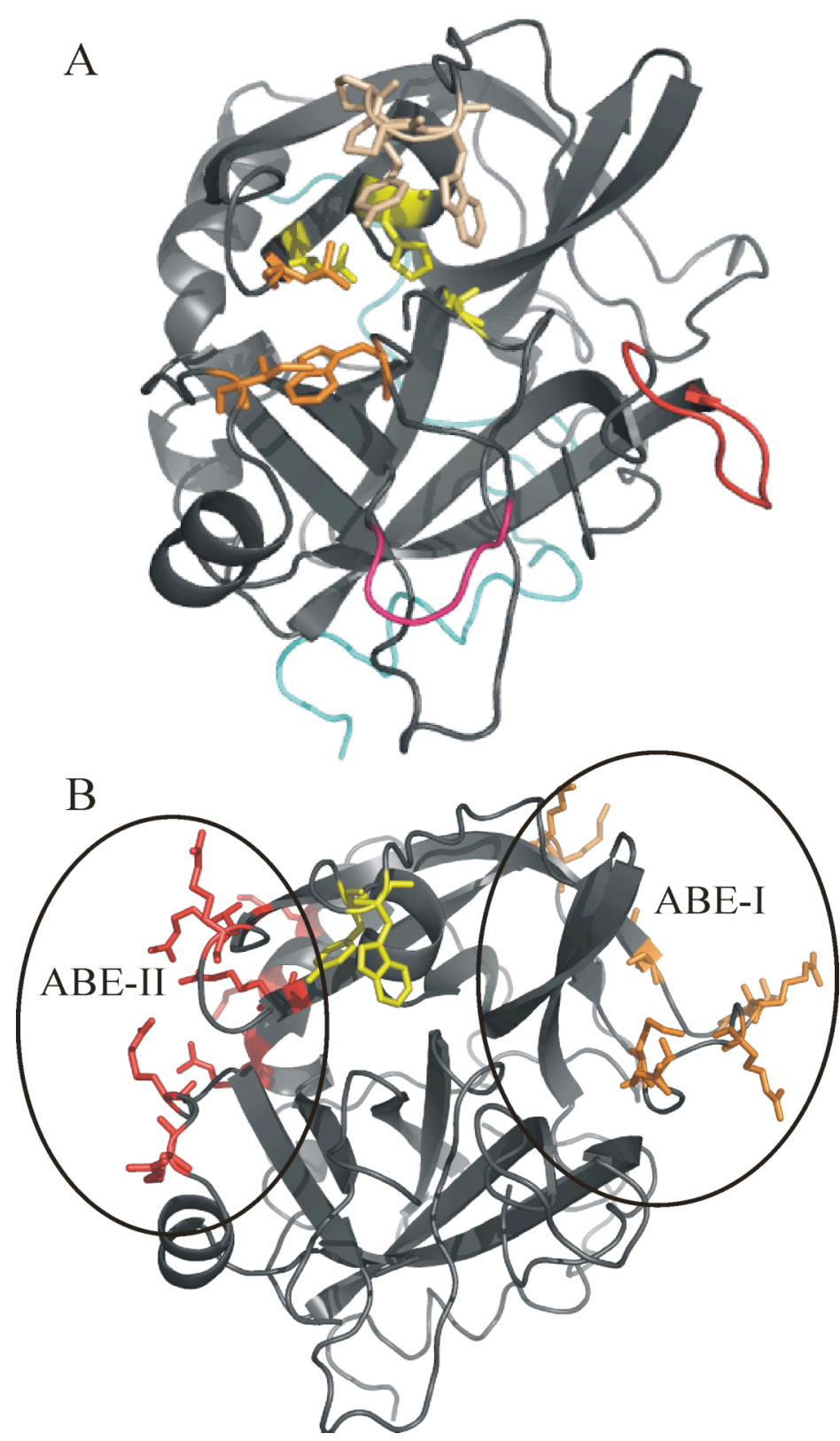

Figure 4: Structure of the serine protease thrombin (1PPB) (51). A. Location of the relevant features of thrombin described in the text. The catalytic triad is in yellow, the 60 loop is in light brown, the residues involved in the $\mathrm{S} 4$ site are orange, the autolysis loop is red, the $\mathrm{Na}^{+}$binding loop is pink and the A-chain is cyan. B. Location of ABE-I and ABE-II in relation to $\mathrm{Y}^{60 \mathrm{a}}-\mathrm{Y}^{60 \mathrm{~d}}$ (yellow). ABE-I is orange and ABE-II is red.

The primary catalytic machinery of all serine proteases is conserved in the catalytic triad of residues $\mathrm{H}^{57}, \mathrm{D}^{102}$, and $\mathrm{S}^{195}$ (Figure 4A) (52). With IIa, the promiscuity observed with trypsin hydrolysis of anything containing an $\mathrm{R}$ or $\mathrm{K}$ at $\mathrm{P}_{1}$ (as long as a proline is not present at $\mathrm{P}_{1}{ }^{\prime}$ ) is not evident. The $\mathrm{P}$ nomenclature system assigns $\mathrm{P}_{1}-\mathrm{P}_{1}{ }^{\prime}$ as the 
hydrolyzable bond. Residues $\mathrm{N}$-terminal to $\mathrm{P}_{1}$ are designated $\mathrm{P}_{2}, \mathrm{P}_{3}$, etc. and residues $\mathrm{C}$ terminal to $\mathrm{P}_{1}{ }^{\prime}$ are $\mathrm{P}_{2}{ }^{\prime}, \mathrm{P}_{3}{ }^{\prime}$, etc. Sites within the enzyme that interact with substrate are given the corresponding number, i.e. $\mathrm{P}_{2}$ binds to the $\mathrm{S}_{2}$ site in IIa.

IIa's specificity is a result of the loops and residues that surround the catalytic triad (reviewed in 48). IIa hydrolyzes the protein backbone primarily after R, although $\mathrm{K}$ and $\mathrm{F}$ at the $\mathrm{P}_{1}$ position can also act as less efficient substrates. At the $\mathrm{P}_{2}$ position, IIa prefers small and hydrophobic residues that can interact effectively with the 60-loop defined by $\mathrm{Y}^{60 \mathrm{~A}}, \mathrm{P}^{60 \mathrm{~B}}, \mathrm{P}^{60 \mathrm{C}}$, and $\mathrm{W}^{60 \mathrm{D}}\left(\mathrm{S}_{2}\right.$ site). The $\mathrm{P}_{3}$ position is not a determinant for specificity since this residue does not interact with the IIa surface. The aryl binding site $\left(\mathrm{S}_{4}\right.$ site) is created by $\mathrm{L}^{99}, \mathrm{I}^{174}$, and $\mathrm{W}^{215}$ leading to a preference for hydrophobic, aromatic residues. The $\gamma$-loop, or autolysis loop (149-149E), is a flexible, hydrophilic surface loop that is involved in determining substrate specificity C-terminally to the scissile bond.

To this point, the discussion has focused on $\alpha$-IIa, but several autolytic sites exist in IIa (Figure 3) (reviewed in 49). Since $\alpha$-IIa in vivo is rapidly inhibited by a multitude of serpins, these forms of IIa probably do not have physiologic significance. $\alpha$-IIa that is isolated in solution over time will hydrolyze several bonds. The first such proteolytic event leads to the generation of $\beta$-IIa. Cleavage of $\mathrm{R}^{77 \mathrm{~A}}-\mathrm{N}^{78}$ exposes the peptide bond $\mathrm{R}^{67}-\mathrm{I}^{68}$ to IIa hydrolysis resulting in the excision of the peptide $\mathrm{I}^{68}-\mathrm{R}^{77 \mathrm{~A}}$. Further catalysis of $\mathrm{K}^{149 \mathrm{E}}-\mathrm{G}^{150}$ in the flexible autolysis loop produces $\gamma$-IIa. It should be noted that bovine $\beta$-IIa can be produced by autolysis; however, a non-conservative substitution of $\mathrm{E}$ for $\mathrm{K}$ at position 149E prevents formation of bovine $\gamma$-IIa (51). These autolytic events do not affect catalysis of small chromogenic substrates, but significantly reduces the amount of fibrinogen that is converted to fibrin due to the disruption of fibrinogen binding (53). 
An important allosteric activator of IIa is sodium (54). The binding loop is located near the $\mathrm{S}_{1}$ pocket (see Figure $4 \mathrm{~A}$ ). $\mathrm{Na}^{+}$coordination involves the carbonyl groups of $\mathrm{R}^{221 \mathrm{~A}}$ and $\mathrm{K}^{224}$ as well as four water molecules and the bound cation is situated $15 \AA$ from the active site (55). Additional stabilization of $\mathrm{Na}^{+}$is provided by $\mathrm{D}^{221}$ and $\mathrm{D}^{222}$. When $\mathrm{Na}^{+}$is bound to IIa, the protease is referred to as being in the fast form defined by a more open and rigid structure, while IIa lacking coordinated $\mathrm{Na}^{+}$is in the slow form characterized by a more closed and flexible structure (56). Procoagulation is favored by the fast form, where cleavage of fibrinogen and PAR-1 are significantly enhanced in the presence of $\mathrm{Na}^{+}$(57). Cleavage of PC with thrombomodulin as a cofactor occurs with greater ease in the slow form (58). Since the concentration of $\mathrm{Na}^{+}$remains tightly regulated at $140 \mathrm{mM}$ in the blood circulation, the partition of fast and slow form IIa in vivo will not deviate from a ratio of $3: 2$ based on the $\mathrm{K}_{\mathrm{D}}$ of $110 \mathrm{mM}(54)$. Whether $\mathrm{Na}^{+}$ acts as an allosteric regulator physiologically is an open debate; however, bound $\mathrm{Na}^{+}$ does result in conformational and catalytic changes for IIa $(54,59)$.

Thrombin contains two exosites that are located on opposing faces of the serine protease flanking the catalytic site (51) (Figure 4B). These highly electropositive patches are termed anion binding exosites I and II (ABE-I and ABE-II). An exosite is a ligandprotein interface that is distinct from the residues involved in catalysis. Exosite functions include guiding substrate orientation for catalysis that is more efficient and/or providing a separate cofactor interaction site that either enhances or dampens enzyme reactivity. Selected residues from ABE-I include $\mathrm{K}^{36}, \mathrm{R}^{67}, \mathrm{R}^{73}, \mathrm{R}^{75}, \mathrm{R}^{77 \mathrm{~A}}, \mathrm{~K}^{81}, \mathrm{~K}^{109}, \mathrm{~K}^{110}$, and $\mathrm{K}^{149 \mathrm{E}}$. ABE-I aides in the activation of fibrinogen, PAR-1, FV, FVIII, and FXI, binds the 
inhibitors hirudin and heparin cofactor II, and interacts with thrombomodulin which switches thrombin's specificity toward the anticoagulant PC $(34,48)$.

\begin{tabular}{|c|c|}
\hline Fibrinogen $\gamma^{\prime}$ & ${ }^{408}$ VRPEHPAETEY $_{S}$ DSLY $Y_{S}$ PEDDL $^{427}$ \\
\hline GpIb $\alpha$ & ${ }^{269} \mathbf{D E G D T D L Y}_{\mathbf{S}} \mathbf{D} \mathbf{Y}_{\mathbf{S}} \mathbf{Y}_{\mathbf{S}} \mathrm{PEEDTEG}{ }^{286}$ \\
\hline FVIII & ${ }^{341}$ EEAEDY $_{\mathbf{S}}$ DDDLTDSEM ${ }^{355}$ \\
\hline
\end{tabular}

${ }^{a}$ Anionic residues are in bold.

${ }^{b}$ These human ABE-II binding sequences were taken from the following sources: fibrinogen $\gamma^{\prime}(60,61)$, GpIb $\alpha$ (62), and factor VIII (63).

ABE-II is more electropositive than ABE-I and encompasses $R^{93}, R^{97}, R^{101}, R^{126}$, $\mathrm{R}^{173}, \mathrm{R}^{175}, \mathrm{R}^{233}, \mathrm{~K}^{235}, \mathrm{~K}^{236}$, and $\mathrm{K}^{240}$. The glycosaminoglycan heparin interacts with ABE-II and plays an important role in inhibition of thrombin by the serpins antithrombin (64) and heparin cofactor II through ternary complex formation. FV and FVIII binding to both ABE-I and ABE-II may serve to further enhance activation of these two cofactors (65). A recent crystal structure of haemadin and thrombin demonstrates the necessity of both ABE-II and the active site for this inhibitory interaction (66). On the surface of platelets, the association of GpIb $\alpha$ with ABE-II accelerates thrombin activation of PAR-1 and FXI, while reducing the rate of fibrinopeptide A release from fibrin $(67,68)$. Finally, a C-terminal variant of the fibrinogen $\gamma$ chain (termed the $\gamma^{\prime}$ chain) has been demonstrated to bind to $\operatorname{ABE}-\mathrm{II}(69,70)$. Table 1 shows a selection of sequences from proteins that interact with ABE-II.

In a significant number of the previously described $\mathrm{ABE}$ interactions, sulfonation appears to be an important element in ligand binding to IIa. In 1954, Bettelheim first reported the presence of sulfonation in a blood coagulant protein at $\mathrm{Y}^{6}$ of the bovine fibrinogen $B \beta$ chain (71). This modified residue probably aids in interaction of the $B \beta$ 
chain with the extended active site region, and possibly ABE-II. Other well known sulfonated ligands are the ABE-I binding active site inhibitor hirudin (72) and the ABE-II targeting heparin sulfate (73). The ABE-II binding sequences of $\gamma^{\prime}$ chain $(60,61)$, GpIb $\alpha$ (62), and FVIII (63) all display an intense clustering of $Y_{S}, D$, and $E$ (Table 1).

The conformational features associated with the interaction of the acidic C-terminal tails of fibrinogen and GpIb $\alpha$ with ABE-II are described in later chapters. Both of these proteins utilize ABE-II for recruitment of IIa to the site of vascular injury and clot formation. In addition, the proteins may play a role as cofactors in the cleavage of IIa's target proteins. The following sections describe these two proteins in further depth.

\section{Glycoprotein Ib $\alpha$}

Residing on the surface of platelets is the transmembrane receptor complex GpIb $\alpha / \beta$-GpIX-GpV (74-77). The importance of this protein complex in blood coagulation is highlighted in Bernard-Soulier syndrome, a bleeding disorder resulting from either dysfunctional or low levels of GpIb $\alpha / \beta-G p I X-G p V$. Disulfide bonds covalently link GpIb $\alpha(140 \mathrm{kDa})$ to $\mathrm{GpIb} \beta(27 \mathrm{kDa})$ recently discovered to exist at a 1:2 ratio (78). GpIX $(22 \mathrm{kDa})$ is non-covalently associated with $\mathrm{GpIb} \alpha / \beta$. The final component of the receptor complex is $\mathrm{GpV}(82 \mathrm{kDa})$, which forms a 1:2 complex with GpIb $\alpha / \beta$-GpIX. GpIb $\alpha$ is a member of the leucine rich repeat (LRR) family (Figure 5). The receptor also has the following structural components C-terminally to the LRR domain: a couple of loop structures linked by a disulfide bond, a highly glycosylated mucin-like stem, a transmembrane domain, and a cytoplasmic region $(79,80)$. 
Within the platelet receptor complex, the N-terminal region (1-290) of GpIb $\alpha$ is the major site of ligand binding. The macrophage antigen-I (Mac-I) (81) and the cell adhesion molecule P-selectin (82) bind GpIb $\alpha$ leading to the recruitment of leukocytes to a site of infection resulting in vascular inflammation. GpIba interacts with FXI (83), FXII (84), and HK (85, 86), bringing these proteins to the platelet surface and amplifying coagulation. Adhesion of platelets to sites of damaged tissue is the primary role for GpIb $\alpha$ binding to vWF under high-shear. When vWF interacts with GpIb $\alpha$, the transmembrane complex is primed to begin intracellular signaling achieving an increase in $\mathrm{Ca}^{2+}$ in the cytosol, degranulation, rearrangement of the cytoskeleton, and activation of the platelet aggregating integrin $\alpha \operatorname{IIb} \beta 3(75,77)$. GpIb $\alpha$ assists thrombin in the activation of PAR-1 (67) and FXI (83). GpIb $\alpha$ may also aid cleavage of GpV by thrombin, though the exact function of $\mathrm{GpV}$ proteolysis has yet to be established. GpV could sterically hinder thrombin binding to and activation of platelets $(87,88)$ and/or be involved in the adhesion of platelets to collagen (89).

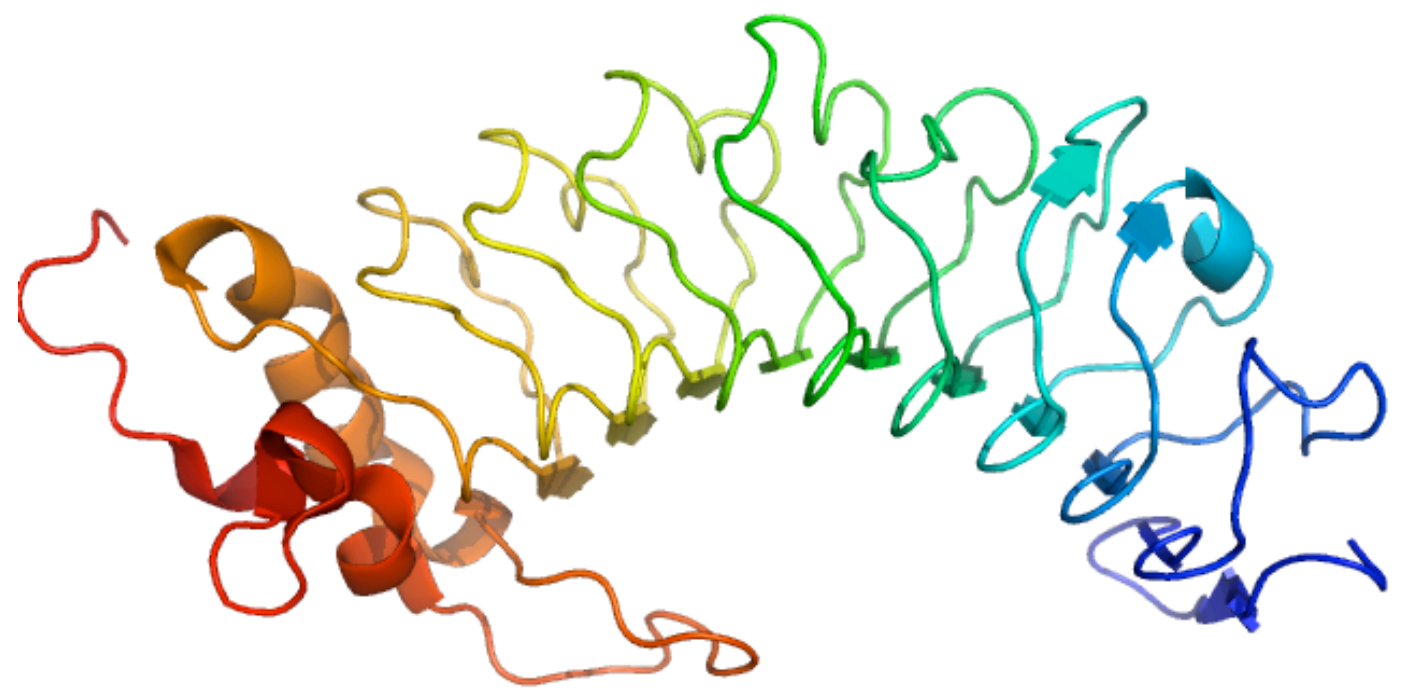

Figure 5: Structure of glycoprotein Ibo (1-266) (1P9A) (96). The highly anionic C-terminal tail (269-286) is not modeled in this structure. The most prominent feature of the structure is the leucine rich repeat domain that forms the curvature of the protein. 
Considerable interest has been directed toward understanding in more detail the nature of the GpIba-IIa interaction in solution (90). The sequence within GpIb $\alpha$ that is responsible for binding thrombin has been localized to the $\mathrm{C}$-terminus of the $\mathrm{N}$-terminal fragment (1-290) between residues $\left({ }^{269}\right.$ DEGDTDLY $_{S}$ DY $Y_{S} Y_{S}$ PEEDTEGD ${ }^{287}$ ) (Table 1) (91). Mutagenesis studies have identified the ABE-II residues $\mathrm{R}^{93}, \mathrm{R}^{97}, \mathrm{R}^{101}, \mathrm{R}^{233}, \mathrm{~K}^{236}$ and $\mathrm{K}^{240}$ as playing a role in binding, while the ABE-I residues $\mathrm{R}^{67}, \mathrm{R}^{73}, \mathrm{R}^{75}, \mathrm{R}^{76}$, and $\mathrm{R}^{77 \mathrm{~A}}$ did not appear to be involved in the interaction $(23,68,92)$. In the mutagenesis work, the indicated residues were usually mutated to alanine and the effect of the mutation on the $\mathrm{K}_{\mathrm{D}}$ was determined.

Though the evidence for ABE-II as the primary destination for GpIb $\alpha$ is strong, others have identified ABE-I as the site of interaction through competitive inhibition with hirudin but not with heparin (93-95). Yet, heparin inhibits GpIba binding to IIa's ABEII and not hirudin (68). Adding to the confusion, two independent crystal structures of IIa-GpIba (residues 1-279/284) were published in Science (96, 97). The Dumas structure displays GpIba residues 269-279 exclusively interacting with ABE-II, while the Celikel structure illustrates residues 269-284 binding to both ABE-II and ABE-I through IIa dimerization. The reasons for the discrepancies in results have yet to be resolved.

The $K_{D}$ values calculated for this interaction are highly salt dependent, which is characteristic of ABE-II binding. At $100 \mathrm{mM} \mathrm{Na}^{+}$, the $\mathrm{K}_{\mathrm{D}}$ ranges from $102 \mathrm{nM}$ to 187 $\mathrm{nM}$, while at $150 \mathrm{mM} \mathrm{Na}^{+}$this value increases to $1 \mu \mathrm{M}(68,92)$. Li et al. calculated a salt dependence of $\Gamma_{\text {salt }}=-4.2$, which indicates at least 4 salt bridges for GpIb $\alpha$-IIa (68). This value is in good agreement with an independent assessment of the salt dependence for GpIb $\alpha-I I a(98)$, as well as for the nonspecific ABE-II ligand heparin (99). 
The efficiency of thrombin catalysis is affected by GpIb $\alpha$ exosites binding. Release of fibrinopeptide A (FpA) is noncompetitively inhibited by GpIba (68), while PAR-1 hydrolysis is enhanced by GpIba binding to IIa (67). Curiously, other groups have attributed GpIba inhibition of FpA hydrolysis to competitive inhibition at ABE-I (100, 101). Based on recent mutagenesis data involving IIa, it seems more likely that inhibition is occurring through ABE-II and not ABE-I $(68,92)$. GpIb $\alpha$ leads to decreased catalysis at $\mathrm{R}^{372}$ in FVIII presumably due to competition for binding at ABE-II (65). These examples emphasize the importance of ligand binding to ABE-II on catalytic activity and the allosteric nature of the interaction.

Though GpIb $\alpha$ plays an important role in platelet aggregation, the protein fibrinogen provides an equally important function in clot formation. Like GpIba, fibrinogen has been demonstrated to bind ABE-I and ABE-II. In this instance, solid data exists illustrating that fibrinogen interacts with both exosites, perhaps simultaneously.

\section{Fibrinogen}

The large soluble glycoprotein fibrinogen $(340 \mathrm{kDa})$ is a dimer comprised of three domains $\left(\mathrm{A} \alpha \mathrm{B} \beta \gamma_{\mathrm{A}}\right)_{2}$ existing as coiled coils in the shape of a dumbbell $(35,102)$ (Figure 6). The N-termini of all six polypeptide chains are located in the central E-region of fibrinogen, supplying a low affinity binding site for thrombin via ABE-I (103). The Ctermini of the polypeptide chains reach laterally from the E-region to form the flanking D regions. During coagulation, thrombin cleaves fibrinopeptides A and B from the Nterminus of $\alpha$ and $\beta$ chains, respectively. This action converts fibrinogen to fibrin $\left(\alpha \beta \gamma_{\mathrm{A}}\right)_{2}$, initiating formation of the noncovalently associated soft clot. 
There are two variants of the $\gamma$ chain C-terminus extending outward from the $\mathrm{D}$ regions $(104,105)$. The $\gamma_{\mathrm{A}}$ chain terminates with four amino acids $\left({ }^{408} \mathrm{AGDV}^{411}\right)$ and interacts with platelets through residues 400-411 (106). The $\gamma^{\prime}$ chain results from a mRNA splice variant due to alternative processing and replaces the last four residues of the $\gamma_{\mathrm{A}}$ chain with an insertion of 20 amino acids $\left({ }^{408}{ }^{\text {VRPEHPAETEY }}\right.$ DSLY $\left.{ }_{\mathrm{S}} \mathrm{PEDDL}^{427}\right)$ $(107,108)$ (Table 1$)$. About $7 \%$ to $15 \%$ of all fibrinogen exist as the $\gamma_{\mathrm{A}} / \gamma^{\prime}$ heterodimer as opposed to the $\gamma_{\mathrm{A}} / \gamma_{\mathrm{A}}$ homodimer $(109,110)$.

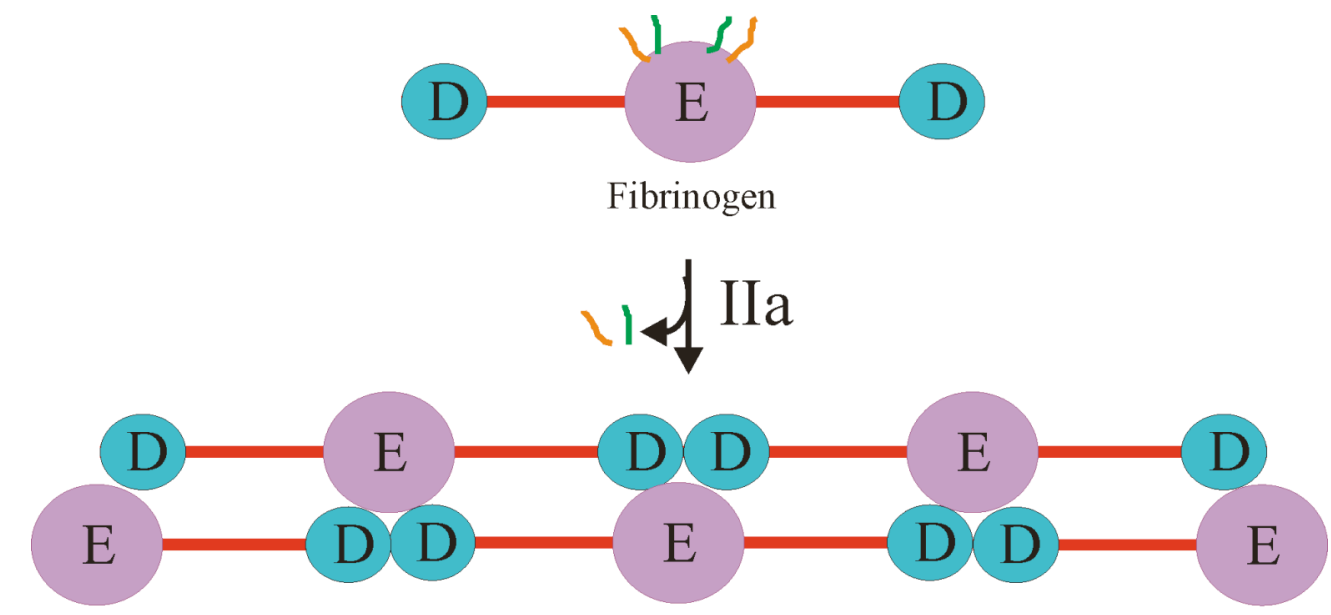

Fibrin and Soft Clot Formation

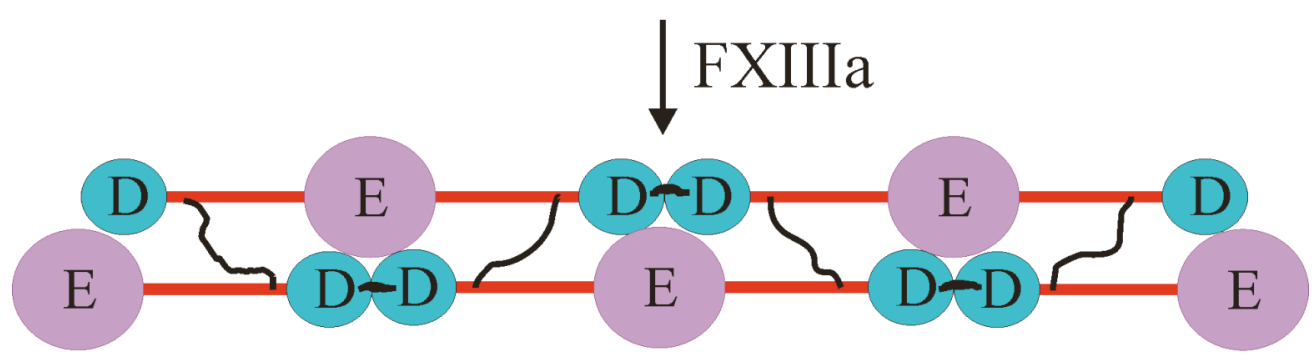

Crosslinked Hard Clot

Figure 6: Schematic diagram depicting the structure of fibrinogen and clot formation. Thrombin cleavage of fibrinopeptides A and B (orange and green) lead to soft clot formation and FXIIIa catalyzes covalent intermolecular crosslinks making the clot more resistant to fibrinolysis.

Meh et al. demonstrated that the D-region of fibrinogen $\gamma_{\mathrm{A}} / \gamma^{\prime}$ contained a high affinity binding site for thrombin with a $\mathrm{K}_{\mathrm{D}}$ of $0.26 \mu \mathrm{M}$ (111). Further work implicated the $\gamma^{\prime}$ residues $\mathrm{A}^{414}$ - $\mathrm{L}^{427}$ as essential to the high affinity interaction, although the exact 
location of the $\gamma^{\prime}$ chain interface on thrombin was unclear (60). Subsequent competitive fluorescent binding studies localized $\gamma^{\prime}$ peptide binding to ABE-II with a $\mathrm{K}_{\mathrm{D}}$ in the $0.63-$ $2.2 \mu \mathrm{M}$ range for residues $\mathrm{V}^{408}-\mathrm{L}^{427}(69,70)$. Replacement of the $\mathrm{Y}_{\mathrm{S}}$ with $\mathrm{F}$ in the $\gamma^{\prime}$ region of fibrin abolishes IIa binding (69). A combination of heparin, hirudin fragments, and DNA aptamers targeting ABE-I and II were employed to verify the site of interaction $(69,70)$. An aptamer is a short oligonucleotide sequence (20-30 bases) that has been selected to bind a target protein with high affinity (112).

The presence of elevated levels of the heterodimer $\gamma_{A} / \gamma^{\prime}$ has been linked to increased incidence of cardiovascular diseases. As with GpIb $\alpha$, the $\gamma^{\prime}$ chain decreases the rate of FpA hydrolysis (113) in this case leading to a fibrin network that possesses many more offshoots with thinner fibers $(113,114)$. Fibrin clots formed in this manner are less susceptible to fibrinolysis $(110,113)$. Fibrin enhanced FXIII activation is slightly slower when the $\gamma^{\prime}$ chain binds to ABE-II (113) suggesting that the levels of $\gamma^{\prime}$ chain variant present in fibrinogen has a significant impact on the regulation of coagulation (115).

The $\gamma^{\prime}$ chain of fibrinogen has also been demonstrated to be a carrier of plasma Factor XIII $\left(\mathrm{A}_{2} \mathrm{~B}_{2}\right)$ via the FXIII $\mathrm{B}_{2}$-subunits (116). Like fibrinogen, IIa also activates FXIII at the conclusion of procoagulation. Several issues have yet to be resolved regarding FXIII activation and substrate recognition. For instance, a high degree of similarity exists between the structures of FXIII and FXIIIa (117-119). Therefore, the conformational transition resulting from activation that enables FXIIIa to perform catalysis is unclear. In the following section, the role FXIII plays in blood coagulation is described in more detail, as well as some of the questions that need to be addressed in relation to this transglutaminase. 


\section{The Transglutaminase Factor XIII}

FXIII $(83 \mathrm{kDa})$ shares the same primarily $\beta$ sheet architecture of the other known transglutaminases (TGases) (119). The five principal structural elements of the TGase family are depicted in Figure 7A: the activation peptide (1-37), the $\beta$ sandwich (residues 38-183), the catalytic core (residues 184-515), and the $\beta$ barrel 1 (residues 516-627) and $\beta$ barrel 2 (residues 628-731) domains. The active site architecture for both cysteine proteases and TGases are quite similar, consisting of the catalytic triad $\mathrm{C}^{314}, \mathrm{H}^{373}$, and $\mathrm{D}^{396}$ (FXIII numbering) (Figure 7B); however, the proposed mechanism of TGases is the reverse of cysteine proteases $(119,120)$. The only TGase to exist as a dimer is FXIII, with key interchain interactions between $\mathrm{K}^{113}-\mathrm{D}^{367^{\prime}}, \mathrm{K}^{257}-\mathrm{E}^{401^{\prime}}$, and $\mathrm{R}^{260}-\mathrm{D}^{404^{\prime}}$ (Figure 7B).

FXIII circulating in the plasma possesses an additional dimer that serves a regulatory function. Upon activation of FXIII by thrombin, the regulatory $\mathrm{B}_{2}$ subunits dissociate from the catalytic $\mathrm{A}_{2}$ subunits in the presence of $1 \mathrm{mM} \mathrm{Ca}^{2+}(121)$. In the platelets and the placenta, FXIII lacks the $\mathrm{B}_{2}$ subunits and is termed cellular FXIII (122). The $\mathrm{A}_{2}$ dimer from plasma and cellular FXIII is equivalent in structure and function.

Catalytically competent FXIIIa is achieved when IIa cleaves the N-terminal activation peptide at $\mathrm{R}^{37}-\mathrm{G}^{38}$ and at least $1 \mathrm{mM} \mathrm{Ca}^{2+}$ is present (referred to as FXIIIa ${ }^{\mathrm{IIa}}$ ) $(123,124)$. FXIIIa catalyzes the formation of isopeptide bonds between Q and K residues and the catalytic mechanism is illustrated in Figure 8 (125). These covalent bonds act to stabilize the growing fibrin clot. Furthermore, FXIIIa aids in protecting the clot from plasmin degradation through the incorporation of substrates, like $\alpha_{2}$ antiplasmin (126), to the fibrin matrix. After proteolysis by IIa, the activation peptide remains noncovalently associated with FXIIIa and transverses both monomers (Figure 7A) (118). A 
secondary thrombin cleavage site is located near the division of the catalytic core and $\beta$ barrel 1 (127). Proteolysis of $\mathrm{K}^{513}-\mathrm{S}^{514}$ reduces the affinity of FXIIIa for Q and K containing substrates, however the catalytic machinery of the TGase is not perturbed (128). Curiously this cleavage has no effect on FXIIIa binding to fibrin (128).

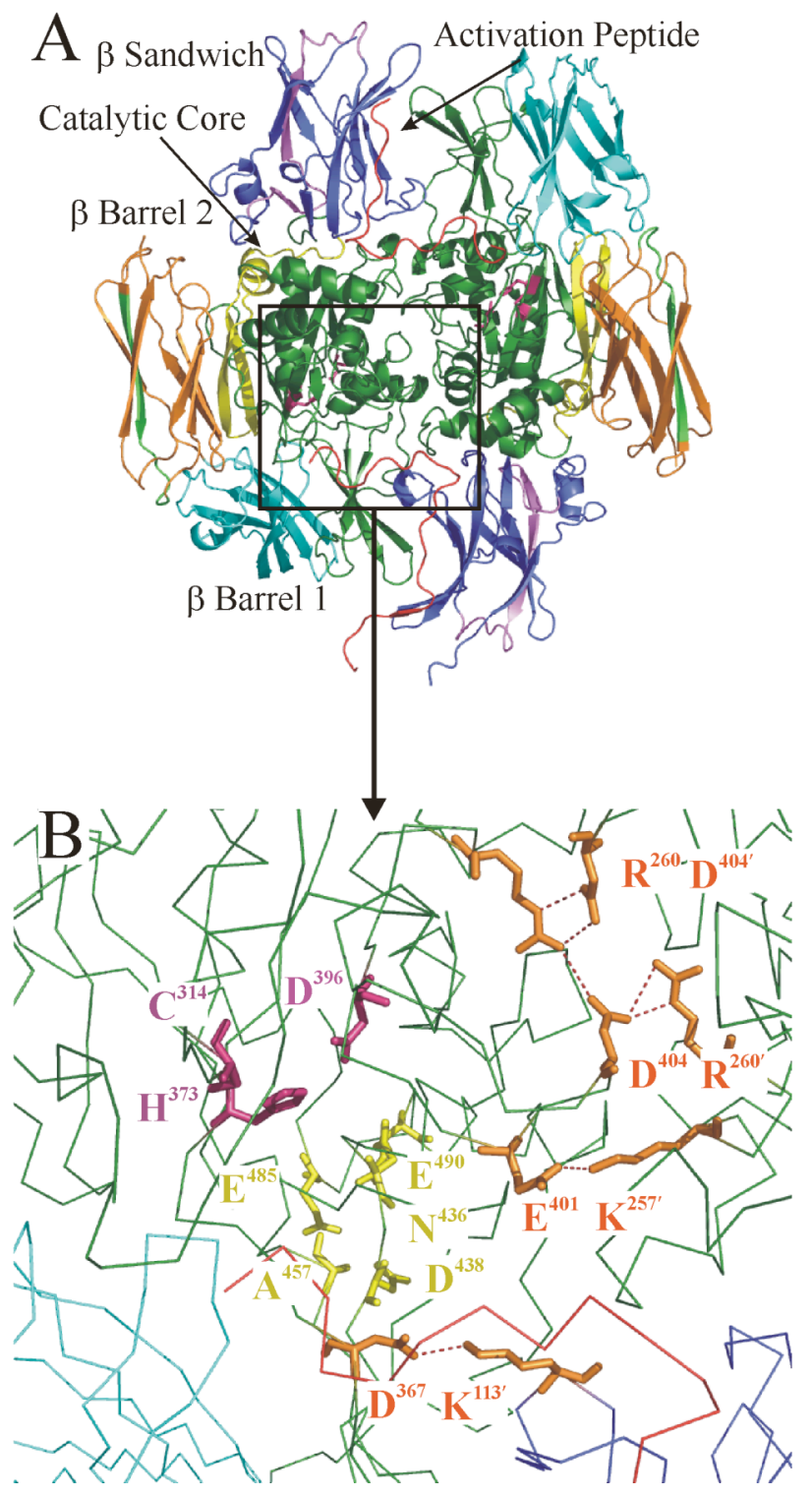

Figure 7: Structure of the transglutaminase Factor XIII (1FIE) (118). A. FXIII depicted as the $\mathrm{A}_{2}$ dimer. Regions include the activation peptide (red), $\beta$ sandwich (blue), catalytic core (green), $\beta$ barrel 1 (cyan), and $\beta$ barrel 2 (orange). The catalytic triad $\left(\mathrm{C}^{314}, \mathrm{H}^{373}\right.$, and $\left.\mathrm{D}^{396}\right)$ is in pink. The two Q substrate recognition sites peptide 4 ( $\beta$ sandwich) and peptide 7 (catalytic core) are purple and yellow respectively. The potential $\mathrm{K}$ substrate recognition site in $\beta$ barrel 2 is light green. $B$. Expanded view of the catalytic core and the dimer interface. Depicted in the figure are the active site (violet), the $\mathrm{Ca}^{2+}$ binding site (yellow), and key interactions at the dimer interface (orange). 
Alternatively, high concentrations of $\mathrm{Ca}^{2+}(>50 \mathrm{mM})$ or of monovalent cations $\left(>150 \mathrm{mM}\right.$ ) with $1 \mathrm{mM} \mathrm{Ca}^{2+}$ can non-proteolytically activate FXIII (referred to as FXIIIa $\left.{ }^{\mathrm{Ca}}\right)(129,130)$. Only one $\mathrm{Ca}^{2+}$ binding site (Figure 7B) has been observed $\left(\mathrm{N}^{436}\right.$, $\mathrm{D}^{438}, \mathrm{~A}^{457}, \mathrm{E}^{485}$, and $\mathrm{E}^{490}$ ) with a $\mathrm{K}_{\mathrm{D}}$ of $\sim 100 \mu \mathrm{M}(117)$, though the existence of several more low affinity sites has been supported by equilibrium dialysis (131) and ${ }^{43} \mathrm{Ca} \mathrm{NMR}$ (132). In addition, several other cations have been demonstrated to interact with FXIII, including magnesium, strontium, manganese, ytterbium, terbium, and zinc $(117,133$, 134). In fact, Fox et al. solved a structure of FXIII with ytterbium bound at the dimer interface chelated by $\mathrm{D}^{270}$ and $\mathrm{E}^{272}$ from both monomers (117).

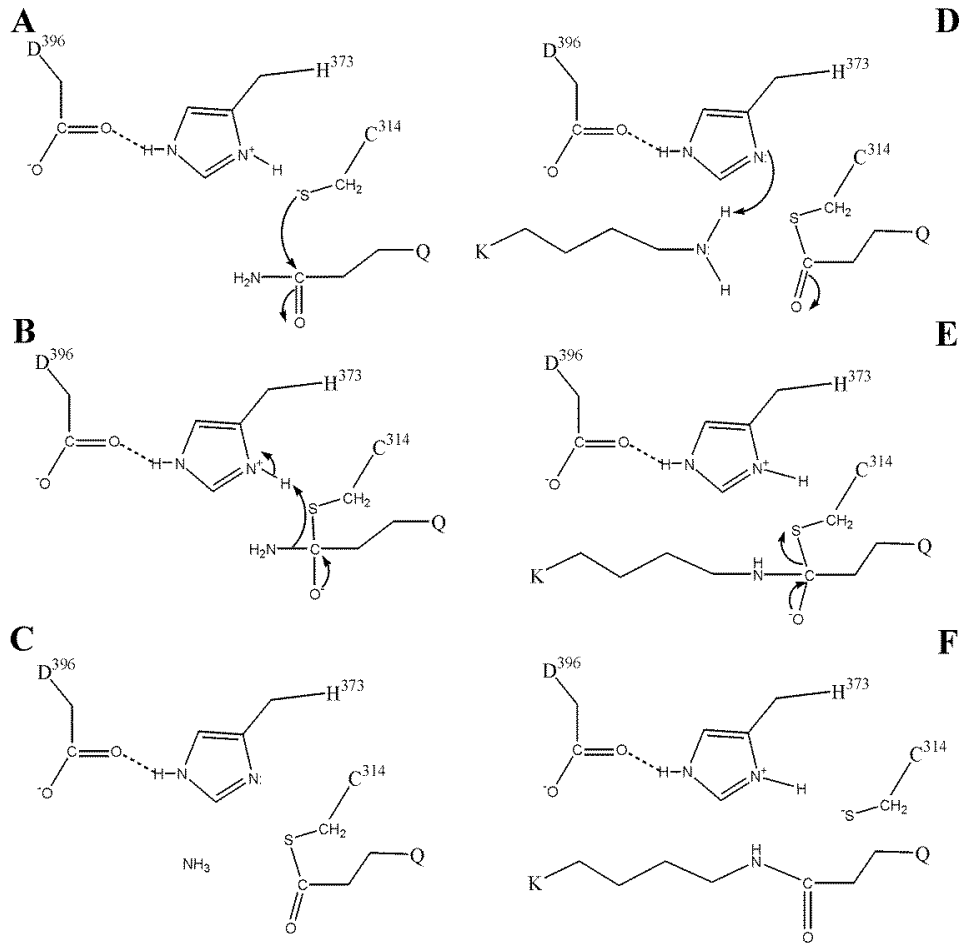

D

Figure 8: Proposed catalytic mechanism of transglutaminases (125). A. The active site thiol attacks the side chain carbonyl of the acyl donor glutamine. B. Formation of the first tetrahedral intermediate. The primary amine abstracts a proton from histidine and leaves as ammonia. C. Formation of the acyl-enzyme intermediate. D. The active site histidine pulls a proton from the donor lysine substrate, which then attacks the carbonyl of the acylF enzyme intermediate. E. Formation of the second tetrahedral intermediate. In this case, the thiol group leaves regenerating the active site for the next crosslinking reaction. $F$. The product of a transglutaminase reaction is an $\varepsilon(\gamma$ glutamyl)lysyl crosslink.

Pinpointing FXIIIa substrate specificity remains an elusive goal. Investigations in the past have focused on primary sequence specificity (135-137) and substrate access to the active site for the Q-containing substrate (138). As far as the $\mathrm{K}$ containing substrate, some work has examined sequence specificity surrounding the reactive lysine residue 
$(139,140)$. In addition to the primary amine in the side chain of $\mathrm{K}$, most other primary amines will complete the TGase reaction (141). The absence of a primary amine will lead to deamidation in the presence of water $(137,142,143)$. These studies have ultimately reached the conclusion that determinants for substrate specificity not only exist near the reactive residues, but also further away from the catalytic triad in potential exosites. An ideal substrate for FXIIIa, or any TGase, would probably be located within a pliable region of a protein, such as surface loops or sequences near the $\mathrm{N}$ - or $\mathrm{C}$ terminus that can reach into the deeply buried catalytic core $(138,144)$.

Several prospective FXIIIa exosites have been proposed. Two potential Q substrate exosites named peptides 4 (72-97) and 7 (190-230) are located within the $\beta$ sandwich and the catalytic core, respectively (Figure 7A) (145). Peptides derived from these sequences competitively inhibit Q substrate catalysis. Within the $\beta$ barrel 2, the antibody 5A2 targets residues 646-658 and acts as a competitive inhibitor of $\mathrm{K}$ substrate incorporation. Such $\beta$ barrel 2 residues may thus be part of a K substrate exosite (146).

Interestingly, alkylation of $\mathrm{C}^{314}$ by a small molecule like iodoacetamide (IAA) occurs only after activation $(124,147-149)$. Yet, the X-ray structures of the zymogen (119), FXIIIa ${ }^{\text {IIa }}$ (118), and FXIIIa ${ }^{\mathrm{Ca}}$ (117) are lacking in significant RMSD deviations (118). These observations led Yee et al. to hypothesize that the inactive and active conformers exist in equilibrium (117). Since the inactive conformer is favored during crystallization, FXIIIa interaction with a substrate or inhibitor may favor crystallization of the active conformer (117). 


\section{$\underline{\text { Research Goals }}$}

A common thread is shared by all projects described in the following chapters. Specifically, the research focuses on the implementation of solution-based methods to better characterize the conformational features associated with ligand binding to the proteins thrombin and FXIIIa. With that in mind, these investigations can be segmented into two separate projects. The first endeavor examines peptide binding to thrombin's

ABE-II, which can be further broken down into studies involving the complexes $\gamma^{\prime}$ chain (410-427)-IIa and Gplba (269-286)-IIa. The second project aims to explore the inhibition of FXIIIa with a newly developed peptide based inhibitor, perhaps yielding new insight into FXIIIa substrate recognition.

\section{$\underline{\text { Rationale for Studying Peptide Binding to Thrombin's ABE-II }}$}

One of the original treatments to promote anticoagulation is the administration of heparin, which interacts at ABE-II $(73,150-152)$ and enhances antithrombin (AT) (64) and heparin cofactor II (153) inactivation of thrombin. Heparin is used clinically to treat myocardial infarction, atrial fibrillation, and deep-vein thrombosis. Unfortunately, the presence of heparin can lead to inhibition of a multitude of other coagulation proteins including FXa (which is also a therapeutic target of heparin) (154), FIXa (155), FXIa (156), FXIIa (157) and vWF (158). In addition to anti-coagulation activities, heparin also binds to circulating plasma proteins (159) and endothelial cells (160). Furthermore, heparin has been shown to promote bone loss (161) and induce thrombocytopenia (low levels of circulating platelets) (162). These heparin side effects vary from individual to individual; therefore levels of heparin dosage must be carefully monitored during 
treatment (163). The difficulties in administrating heparin have led to the development of several heparin-derived drugs and other anticoagulants (164).

Peptides provide a potential route for specifically targeted therapeutic utility. Drugs designed from naturally occurring protein ligands of thrombin could avoid many of the side effects resulting from heparin treatment (163). The efficacy of peptide-based anticoagulants has been demonstrated with the ABE-I binding inhibitors hirudin and bivalirudin $(165,166)$. At the present time, a peptide-based anticoagulant that targets ABE-II has not been developed. By examining the conformational features associated with $\gamma^{\prime}$ peptide (410-427) and GpIb $\alpha$ (269-286) binding to ABE-II, an ABE-II targeting peptide with anticoagulant properties may be developed employing the unique structural elements characteristic of these complexes.

The work described in Chapter IV details the $\gamma^{\prime}$ peptide (410-427)-IIa interaction utilizing NMR and MALDI-TOF MS (167). At the time this research was initiated, a crystal structure demonstrating the interaction of the $\gamma^{\prime}$ peptide with IIa had not been reported, though the binding site was localized to $\operatorname{ABE}-\mathrm{II}(69,70)$. The length of the $\gamma^{\prime}$ peptide substitution for $\gamma_{\mathrm{A}}$ in fibrinogen is twenty amino acids. Therefore, once bound to thrombin, the $\gamma^{\prime}$ peptide may potentially adopt secondary structural elements, i.e. helix formation, turn structures etc., not favored when the peptide is free in solution.

From thrombin's perspective, the residues within ABE-II that provide the $\gamma^{\prime}$ peptide interface had not been elucidated. Crystal structures of heparin (168), prothrombin fragment II (169), GpIb $\alpha(96,97)$, and haemadin (66) binding to ABE-II provide an important starting point for predicting where the $\gamma^{\prime}$ peptide interacts at ABE-II. All structures highlight $\mathrm{R}^{93}, \mathrm{R}^{101}$, and $\mathrm{K}^{240}$ as essential ABE-II binding residues, suggesting 
that the $\gamma^{\prime}$ peptide also utilizes these positively charged residues when binding to thrombin. Another aspect of ABE-II binding that remains to be further explored is whether an allosteric connection exists from ABE-II to ABE-I. Some reports suggest such a pathway is present in thrombin $(70,170)$, while other work appears to dismiss such a possibility (171). Since thrombomodulin binding to ABE-I has been demonstrated to perturb the dynamics of deuterium incorporation at ABE-II $(172,173)$, perhaps the reverse is true for ligand binding to ABE-II.

With those questions in mind, research was initiated to further explore the $\gamma^{\prime}$ peptide-IIa relationship. The project implemented 1D line broadening NMR and 2D transferred NOESY NMR (detailed in Chapter II) to describe the ligand protein interaction from the $\gamma^{\prime}$ peptide's perspective. Using these techniques, the important protons that contact thrombin's surface were identified in addition to several key secondary conformational features associated with $\gamma^{\prime}$ chain binding to IIa. By using MALDI-TOF MS and hydrogen deuterium exchange (HDX) (discussed in Chapter III), the effects of the $\gamma^{\prime}$ peptide on the dynamics of HDX for IIa were examined. These experiments were performed to more accurately localize $\gamma^{\prime}$ peptide binding within ABEII and to observe potential allostery associated with ligand binding to ABE-II.

Chapter V focuses on the interaction between GpIba and IIa. Two crystal structures of GpIba bound to IIa were solved simultaneously $(96,97)$. As discussed earlier in the chapter, thrombin's interaction with ABE-II appears to be similar in both structures, while the second IIa binds at differing interfaces on GpIba. The anionic Cterminal tail in the Dumas et al. structure ended at $\mathrm{Y}_{\mathrm{S}}{ }^{279}$ due to insufficient electron density to model these residues (96). Conversely, the Celikel et al. structure terminates at 
$\mathrm{T}^{284}$, with the C-terminus spanning both thrombin molecules and interacting with ABE-II and ABE-I in an extended conformation (97). The Celikel structure suggests that the Cterminal fragment of GpIb $\alpha$ may induce dimerization of thrombin. Due to the high degree of similarity between the ABE-II binding sequences of GpIb $\alpha$ and the $\gamma^{\prime}$ peptide (see Table 1), perhaps these anionic, sulfonated regions share conformational elements that direct these residues to interact with ABE-II.

As with the $\gamma^{\prime}$ peptide, research was performed to study the GpIb $\alpha$ (269-286)-IIa interaction in further detail utilizing NMR and HDX coupled with MALDI-TOF MS. 1D and 2D NMR work demonstrate that GpIba (269-286) and the $\gamma^{\prime}$ peptide adopt differing conformations when bound to ABE-II. HDX coupled with MALDI-TOF MS was performed in order to distinguish between ABE-II versus ABE-I binding of the GpIb $\alpha$ peptide, as well as to characterize allostery associated with GpIb $\alpha$ interactions with IIa. Finally, further work was then carried out with analytical ultracentrifugation to observe whether the $\gamma^{\prime}$ peptide or the GpIbo peptide could dimerize IIa in solution.

\section{$\underline{\text { Rationale for Studying Peptide Inhibition of Factor XIIIa }}$}

Examining FXIIIa in solution is a difficult task, since the active TGase is mostly insoluble by nature (as is the soft clot that FXIIIa covalently crosslinks). This insolubility perhaps explains the difficulties in obtaining a structure of the active FXIIIa (117). As mentioned earlier in the chapter, structures have been solved for FXIIIa ${ }^{\text {IIa }}$ (118) and FXIIIa ${ }^{\mathrm{Ca}}$ (117), though without significant RMSD deviations from the zymogen (119). Many studies have demonstrated that activation of FXIII must lead to a conformational change that has been undetected in the reported X-ray structures (124, 
147-149). Fortunately, sufficient quantities of FXIIIa ${ }^{\mathrm{IIa}}$ and FXIIIa ${ }^{\mathrm{Ca}}$ remain in solution in order to probe the conformational changes associated with activation $(174,175)$.

These observations have led to the hypothesis that irreversible inhibitor binding to FXIIIa may capture the TGase in an active conformation (117). Inhibited FXIIIa could potentially be utilized to detail previously unobserved features of FXIIIa substrate recognition and, indirectly, activation. Recently, irreversible inhibitors have been developed for TGase 2 based on natural Q-containing substrate sequences for the enzyme (176). One of the reported peptide inhibitors of the TGase incorporated a glutamine isostere 6-diazo-5-oxo-norleucine $(\mathrm{DON})$ in place of the reactive glutamine. A wellcharacterized substrate for FXIIIa is the K9 peptide (177). The DON moiety has been incorporated into the K9 peptide in order to study the conformational features resulting from inhibitor binding to FXIIIa.

Perturbations in FXIIIa dynamics were further explored by inhibition with K9 DON or iodoacetamide (IAA) (Chapter VI) (178). The investigations rely primarily on MALDI-TOF MS methodology (Chapter III). HDX, limited proteolysis, and differential chemical modification were the strategies used to study the conformational changes associated with FXIIIa inhibition, as well as build upon the earlier work investigating FXIII activation $(174,175)$. The FXIIIa inhibitors K9 DON and IAA both displayed similar perturbations in protein dynamics for regions distant from the active site, perhaps indicating the location of exosites within the TGase. These experiments may also have provided evidence for a potential interaction site for $\mathrm{K} 9 \mathrm{DON}$ within the catalytic core. Though these are low-resolution studies, highly valuable glimpses are being provided on FXIIIa allostery and the presence of putative FXIIIa exosites. 


\section{CHAPTER II}

\section{NUCLEAR MAGNETIC RESONANCE TECHNIQUES: 1D LINE BROADENING AND 2D TRANSFERRED NOESY}

One of the most powerful tools to study molecular structure and dynamics, ligandreceptor interactions, and chemical kinetics is nuclear magnetic resonance spectroscopy (reviewed in 179). Through the application of an external magnetic field, a nuclear spin becomes excited, ultimately allowing the detection of perturbations in electronic environment that are quite subtle. In order to achieve an excited state, radio waves irradiate the sample indicating that excitation requires small amounts of energy. Consequently, the signal to noise in an NMR spectrum is low, thus requiring an abundance of material and multiple scans to enhance resolution. One beneficial aspect of the low energy transition is the long lifetime of nuclear spin excitation, which ranges from msec to sec. It is this feature of an NMR experiment that produces very narrow spectral signals and permits the observance of molecular motion over a large time scale. Most importantly, the long lifetime of nuclear spin excitation confers the ability to transfer resonance energy from one spin to another spin. As relaxation occurs, the energy released by the nuclear spin is measured as a free induction decay (FID), which is converted to a plot of intensity versus frequency by Fourier transform.

The magnetization that is experienced by the nucleus is primarily affected by the density of electrons surrounding the nuclear spin (reviewed in 179). Application of an external magnetic field also causes electrons to precess resulting in the creation of a 
magnetic field that opposes the applied field. This phenomenon leads to shielding of the nuclear spin and is termed chemical shift. Larger electron density equates to lower resonance frequencies of the observed nuclear spin. Therefore, the measured frequency of a nuclear spin not only depends on the type of nuclei studied, but the electronic environment in which the nuclear spin resides, whether due to bonding or proximity.

For amino acids, typical chemical shifts for the observed protons depend on the atom and position of the group within the residue. Since nitrogen is more electronegative than carbon, a backbone amide proton will possess a chemical shift at $\approx 8 \mathrm{ppm}$. The proximity of $\mathrm{H}_{\alpha}$ to the backbone nitrogen results in a resonance at $\approx 4 \mathrm{ppm}$, while $\mathrm{H}_{\delta}$ from leucine resonates further upfield at $\approx 1 \mathrm{ppm}$. This example illustrates the fundamental complexity of 1D spectra for peptides and proteins due to overlap of multiple resonances for similar protons. In order to resolve the spectra of larger polypeptides, NMR experiments are performed in multiple dimensions, where multiple chemical shifts aide in assigning the resonances for the observed peaks.

As with any transition from a ground state to an excited state, energy is eventually released as the system reestablishes equilibrium (reviewed in 179). In NMR, the nuclear spins that are excited experience two types of relaxation. The first relaxation process is termed spin-lattice relaxation, whose time constant is $T_{1}$. As energy transfers from the excited nuclear spin to the environment, or lattice, the original Boltzman population distribution is regenerated as the magnetic moment of the spins are realigned along the static magnetic field. For proteins, the time scale for $T_{1}$ relaxation is around a second, while for smaller molecules, like peptides, $\mathrm{T}_{1}$ times range from 10 to 100 seconds. 
Transverse relaxation, with a time constant of $T_{2}$, does not involve reestablishing thermal equilibrium or demoting excited spins to the ground state (reviewed in 179). $\mathrm{T}_{2}$ relaxation describes the agitation of nuclear spin phase coherence. Three processes dephase transverse magnetization. First, inhomogeneity of the magnetic field within the sample affects the precessional frequency of the excited spins depending on the spatial position of the spin within the inhomogeneous field. To minimize inhomogeneity, modern instruments possess shim coils that serve to increase the homogeneity of the magnetic field. The second way for transverse relaxation to occur is due to the existence of anisotropic electron density that envelops the nucleus (Chemical Shift Anisotropy). Thus, the alignment of the nuclear spin within the magnetic field will actually determine the actual field that is experienced by the spin due to variations in electron shielding. The final type of transverse relaxation is spin-spin relaxation, which describes dipolar coupling between two magnetic dipoles in close proximity.

For protons, dipolar coupling is the primary means for transverse relaxation (reviewed in 179). Thus, molecular motion and, subsequently, molecular weight play an important role in dipolar coupling. The rotational correlation time $\left(\tau_{c}\right)$ refers to the average time a molecule in solution rotates 1 radian, which is about $1 \mathrm{nsec} / 2.6 \mathrm{kDa}$. When the resonance frequency $(\omega)$ associated with the coupled spins is much slower than $\tau_{\mathrm{c}}\left(\omega \tau_{\mathrm{c}}<<1\right)$, isotropic tumbling is observed, the dipolar coupling contribution to the spin energy is zero, and splitting of resonance lines are not observed. This situation is observed for small molecules in solution. For larger molecules, like proteins, the time required to rotate 1 radian is much longer, resulting in shorter $T_{2}$ lifetimes $\left(\omega \tau_{c}>>1\right)$ and presenting a serious impediment for the transfer of magnetization between spins. Despite 
the longer $\tau_{c}$, proteins tend to possess rotational frequencies on the order of $10^{8} \mathrm{~Hz}$, thus, tumbling in solution is sufficiently fast to subvert the splitting of the resonance lines (dipolar coupling is averaged).

Differences in rotational correlation times $\left(\tau_{c}\right)$ between proteins and peptides can be exploited to obtain structural information regarding peptide interactions with a protein (reviewed in 180,181-184). In the 1D spectrum of a peptide interaction with a protein, line broadening of the spectral signal for the nuclei being observed is an indication of contact between that atom and the protein surface. Conformational information can be obtained by performing a $2 \mathrm{D}$ transferred NOESY experiment where 2 protons within $5 \AA$ display a cross peak which can then be employed to determine structural features of the bound peptide. A majority of the NMR work described in the subsequent chapters deals with implementing these two techniques to describe the conformational features associated with two peptides interacting with thrombin's anion binding exosite II.

Protons (or ${ }^{31} \mathrm{P}$ nuclei of phosphorylated peptides) that contact the protein surface (and thus contribute to the binding interface) can be discerned by comparing the 1D spectra of the free peptide versus the bound peptide. Equation 1 illustrates the relationship between $T_{2}, \omega \tau_{c}$, and the difference in resonance linewidth $\left(\pi \Delta \mathrm{v}_{\mathrm{i}}\right)(180)$. Within Equation 1, $g=0.1 \gamma_{\mathrm{i}}^{2} \gamma_{\mathrm{j}}^{2}(\mathrm{~h} / 2 \pi)^{2}\left(\mu_{0} / 4 \pi\right)^{2}$ and the distance between protons is $\mathrm{r}_{\mathrm{j} j}$. Peptides with less than 18 amino acids lack significant secondary structure and tumble

$$
\pi \Delta v_{i}=\frac{1}{T_{2, i}}=g \sum_{j} r_{i j}{ }^{-6}\left[2.5 \tau_{c}+\frac{4.5 \tau_{c}}{\left(1+\omega^{2} \tau_{c}^{2}\right)}+\frac{3 \tau_{c}}{\left(1+4 \omega^{2} \tau_{c}^{2}\right)}\right]
$$


rapidly when free in solution $\left(\omega \tau_{\mathrm{c}}\right.$ approaches 1$)$. In the $1 \mathrm{D}{ }^{1} \mathrm{H}$ or ${ }^{31} \mathrm{P}$ spectra of the free peptide, well-defined, narrow spectral peaks result from a longer $\mathrm{T}_{2}$ time $(180,184)$.

When examining the bound state, the peptide concentration is in molar excess to the protein and the $\mathrm{k}_{\text {off }}$ has to be sufficiently fast to allow the peptides with secondary structural information to rejoin the free peptides in solution. Upon interacting with the surface of an enzyme, the peptide now adopts a conformation with distinct secondary structural features. Nuclei that interact with the enzyme surface experience an increase in $\tau_{\mathrm{c}}$ leading to $\omega \tau_{\mathrm{c}}>>1$. The decrease in the $T_{2}$, coupled to differences in chemical shift arising from the nuclei experiencing a new magnetic environment, lead to line broadening of the peaks in the 1D spectra. Nuclei that do not interact with the surface of the protein display similar spectral linewidths as observed with the free peptide (185).

Figure 9 presents an example of a 1D ${ }^{1} \mathrm{H}$ line broadening experiment. Protons that contact the protein surface are broadened relative to the spectra of the free peptide. In this example, several residues are not involved in the binding interface, particularly the $\mathrm{G}(\sim 8.65 \mathrm{ppm})$ and $\mathrm{T}(\sim 8.2$ ppm) residues, and are illustrated by peaks that remain sharp and well resolved in the absence and presence of the protein. Conversely, the $\mathrm{Y}(\sim 7.83 \mathrm{ppm})$ residue is broadened in the presence of the protein.

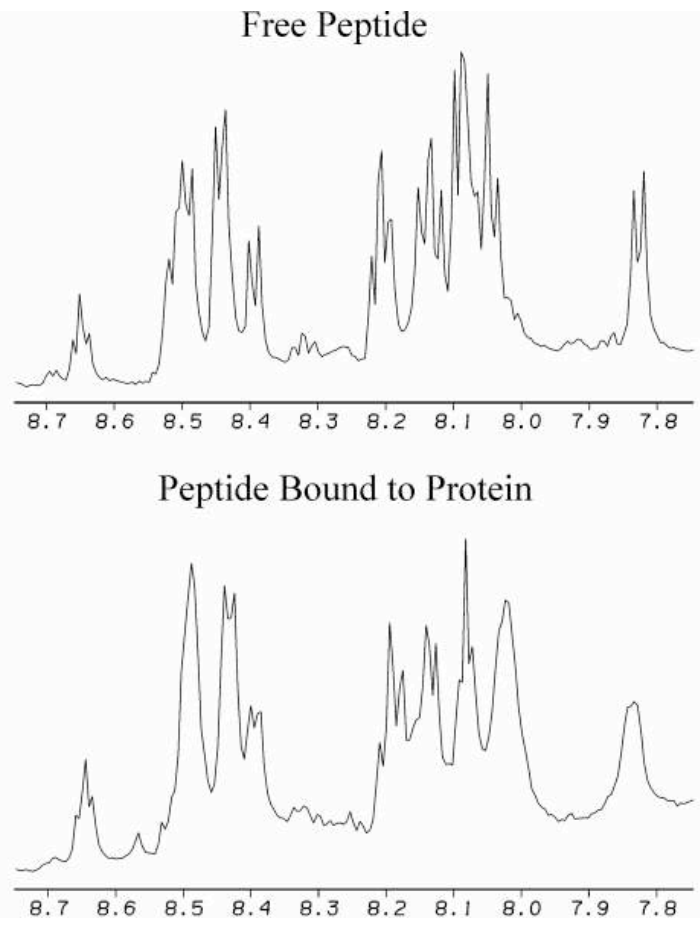

Figure 9: Example of line broadening in 1D NMR spectra. The top spectrum is free unphosphorylated GpIbo (269-286) and the bottom spectrum is of the peptide bound to IIa. 
Due to the overlapping resonances of protons in a 1D spectrum, 2D TOtal Correlation SpectroscopY (TOCSY) NMR is performed in order to assign chemical shifts of similar protons within a peptide. TOCSY NMR takes advantage of a spin lock pulse,

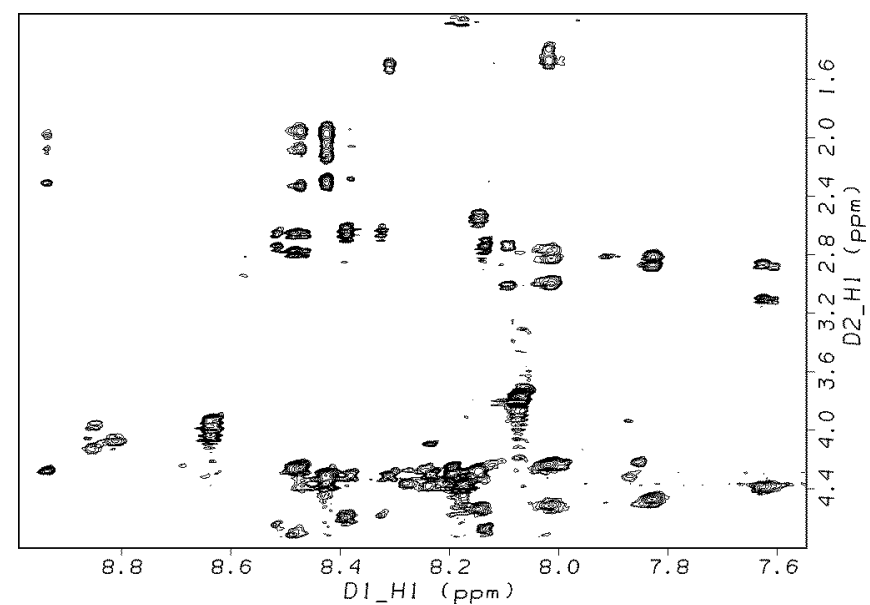

Figure 10: Fingerprint region from a 2D TOCSY spectrum. The spectrum is of unphosphorylated GpIba (269-286) interacting with bovine IIa. On the far left of the spectrum is an example of a spin system for an $\mathrm{E}$ residue $(9 \mathrm{ppm})$. The backbone amide's spin is correlated with the $\alpha$ (4.4 ppm), $\beta$ (2.0 ppm), and $\gamma(2.4$ ppm) proton resonances for this particular $\mathrm{E}$ residue. which enables the connectivity for an entire spin system to be visualized within the $2 \mathrm{D}$ spectrum. Carbonyls within amide bonds demarcate the spin systems of amino acids in a peptide. Therefore, each residue will have a unique set of proton-proton correlations flowing from the backbone amide proton to the side chain protons. Figure 10 displays

the amide to aliphatic proton region frequently referred to as the "fingerprint" region. Cross-peaks representing protons with unique chemical shifts yet are neighbors due to scaler coupling (through bond interactions) appear on opposite sides of the diagonal (for an example of an entire spectrum see Figure 11, which is similar to the layout of a TOCSY spectrum). By assigning the resonances in the 2D TOCSY, the 1D spectrum (the diagonal) becomes rather straightforward to identify.

In addition to line broadening, another NMR technique provides information concerning the conformational features associated with peptide binding: 2D transferred Nuclear Overhauser Effect SpectroscopY (trNOESY). A typical spectrum obtained 


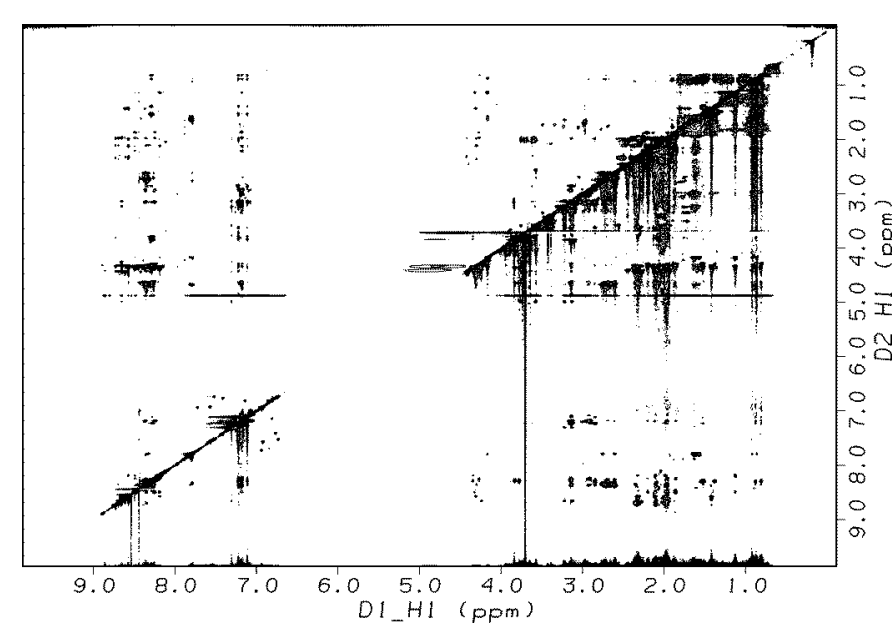

Figure 11: 2D transferred NOESY spectrum. The spectrum is of the $\gamma^{\prime}$ peptide interacting with bovine IIa. during a $2 \mathrm{D}$ experiment is illustrated in Figure 11. Along the diagonal is the $1 \mathrm{D}$ spectrum of the peptide, while crosspeaks representing NOEs between protons flank the diagonal. An NOE develops due to a transfer of magnetization between two protons through space, resulting in

cross-relaxation. Since dipolar coupling depends on the distance between the protons (relaxation rates are proportional to $1 / \mathrm{r}^{6}$ ), only protons within $5 \AA$ will result in a NOE peak. Equation 2 relates the relaxation rate between protons $(\sigma)$ to the interproton distance (r). Thus, NOE intensity can be implemented to calculate a structure for the peptide interacting with a protein (186).

$$
\frac{r_{i j}}{r_{i k}}=\left(\frac{\sigma_{i k}}{\sigma_{i j}}\right)^{\frac{1}{6}}
$$

The $2 \mathrm{D}$ trNOESY NMR experiment also profits from the variations in $\tau_{\mathrm{c}}$ resulting from molecular weight between a peptide and a protein. Once again, a free peptide (of less than 18 residues) possesses $\tau_{\mathrm{c}}$ values of approximately $10^{-10}$ sec leading to $\omega \tau_{\mathrm{c}} \leq 1$. This situation equates to NOEs that are both small and positive or approach zero. The binding of the peptide to a larger protein $\left(\tau_{\mathrm{c}}>10^{-8} \mathrm{sec}\right)$ means $\omega \tau_{\mathrm{c}}>>1$ and the NOEs are large and negative, dominating the $2 \mathrm{D}$ spectrum. Interaction of the peptide with the 
protein surface may result in a defined conformation for the peptide. For a successful experiment, the peptide must be in molar excess to the protein $(\sim 10: 1)$. The $\mathrm{k}_{\text {off }}$ should be sufficiently quick for the peptide to rejoin the free peptide population, allowing another peptide to bind to the protein. If these conditions are met, then reliable information regarding the bound state of the peptide will be obtained (Figure 12).

In instances where the peptide binds too tightly to the enzyme, line broadening and NOEs will not be observed in the spectra. This situation results in less buildup of magnetization intensity within the free peptide population since the peptide remains

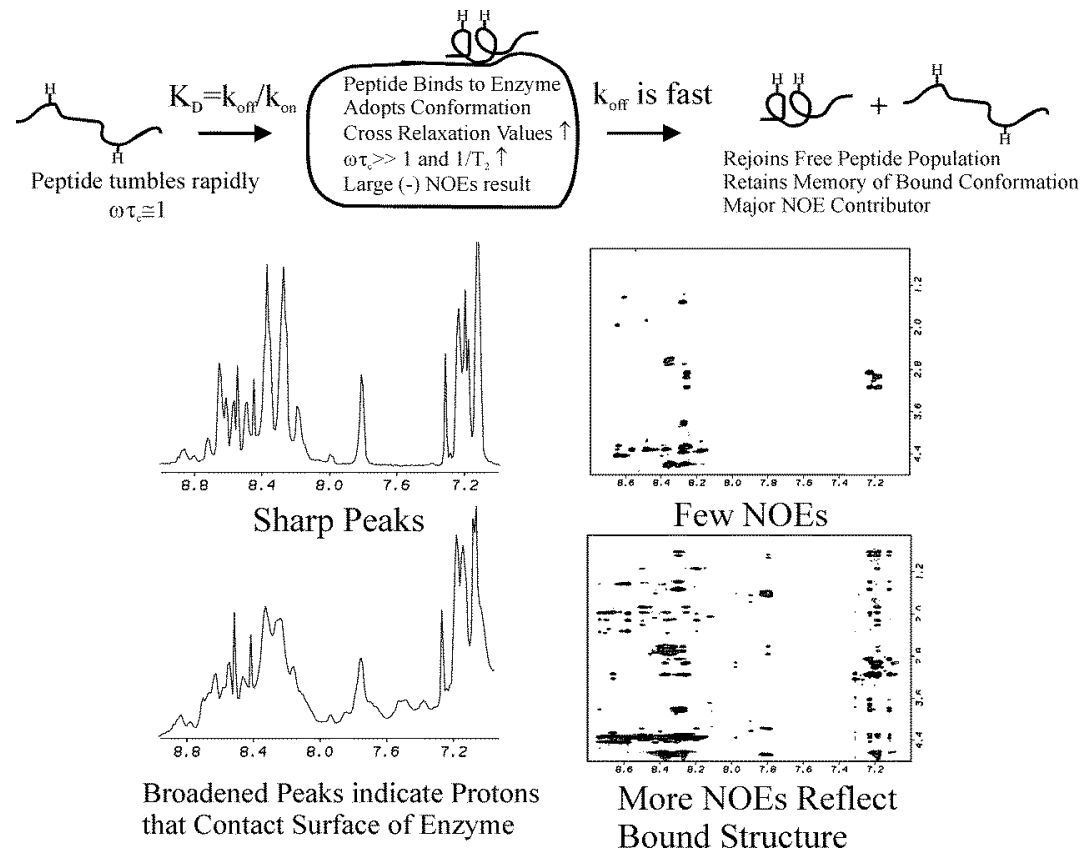

Figure 12: Summary of the 1D line broadening and 2D transferred NOESY techniques. When free in solution, a peptide possesses very little secondary structural features due to lack of constraining factors, such as a protein in which to interact. This situation results in $\omega \tau_{\mathrm{c}} \leq 1$ and longer transverse relaxation $\left(\mathrm{T}_{2}\right)$ times. The $1 \mathrm{D}$ spectrum will display very sharp peaks and the $2 \mathrm{D}$ trNOESY will lack the presence of many discernable NOEs. Introduction of the binding partner, in this case a larger protein, leads to the peptide interacting with protein. Thus, those protons that contact the protein surface adopt the solution characteristics of the larger macromolecule, i.e. $\omega \tau_{c}>>1$, shorter $T_{2}$ times, and large negative NOEs. The peptide now assumes the secondary characteristics associated with binding to the protein. If the $\mathrm{k}_{\text {off }}$ for the peptide is sufficiently fast, then the peptide will take a "memory" of the bound conformation to the free peptide population. This rejoining with the free peptide population results in 1D line broadening for protons that contact the protein surface and a weighted average of the NOES of the free and bound ligands in the 2D trNOESY spectrum. 
bound to the protein. A variety of measures can be implemented to circumvent these situations (reviewed in 184). First, since $\mathrm{k}_{\text {off }}$ is dependent on temperature, raising the temperature may weaken the interaction enough for the bound peptide to dissociate from the protein and retain the characteristics of the bound conformation. Another method involves manipulating the ionic strength or the $\mathrm{pH}$ of the solution, which could interfere with binding to protein enough to increase $\mathrm{k}_{\text {off. }}$ Larger ratios of peptide to enzyme (up to 50:1) create a situation where more peptide is competing for the protein-binding site, thus increasing dissociation. Finally, modifying the residues within the peptide, such as removing phosphorylation from a phosphotyrosine or substituting an $\mathrm{F}$ for a $\mathrm{Y}$, may achieve a similar effect as raising the temperature. Increasing $\mathrm{k}_{\text {off }}$ too much may result in the disappearance of NOEs and line broadening as well, because the rate of interaction with the protein is now faster than the detection limits of the NMR experiment.

Spin diffusion resulting from intermolecular relaxation with the protein can interfere with interpretation of NOEs. In this case, distances between protons appear to be shorter due to dipolar coupling with protein protons leading to spin diffusion across the protein surface. A direct outcome of spin diffusion is a peptide structure that is quite compact when compared to the actual conformation of the ligand. Increasing the peptide to protein ratio may circumvent this situation. Another way around this potential problem is by decreasing the mixing time for the NMR experiment, which discourages indirect transfer of magnetization from the protein to the peptide and thus the appearance of an artificial NOE or an increase in NOE intensity. A comparison of NOEs from spectra acquired at a range of mixing times should display a linear dependence for NOE intensity with mixing time length (184). 
The ultimate advantage of these two NMR techniques, 1D line broadening and 2D trNOESY, rests in the ability to observe, in solution, the conformational characteristics of a ligand bound to a protein. In X-ray crystallography, a static snapshot of a system is captured which could potentially be deceptive with regard to structural information. Crystal packing effects within a lattice, or between neighboring protein molecules, could potentially influence the solved structure and constrain the conformation of a bound ligand. These techniques can observe transient interactions that may not be able to be crystallized due to weak $\mathrm{K}_{\mathrm{D}}$ values.

As of this writing, NMR structures of IIa and FXIII have not been published. The most probable reason for this absence is due to the need of large amounts of isotopically labeled protein, which are required to obtain the NMR structure. Without a reliable E.coli expression system, trNOESY NMR experiments provide a means to observe IIaligand or FXIIIa-substrate interactions in solution with no requirement for large amounts of protein. A glance at the literature reveals that these NMR techniques have been employed to gather an extensive amount of information regarding ligand interactions with IIa's active site and anion binding exosite I $(185,187-199)$. As for FXIIIa, NMR work has focused on examining the interaction of the FXIIIa substrates K9 and $\alpha 2$ antiplasmin binding to the active site $(137,200)$. For the first time, the conformational features associated with peptide binding to IIa's anion binding exosite II have been elucidated in solution and are described in the subsequent chapters (167). 


\section{CHAPTER III}

\section{MATRIX ASSISTED LASER DESORPTION IONIZATION TIME-OF-FLIGHT MASS SPECTROMETRY AND HYDROGEN DEUTERIUM EXCHANGE}

As discussed in the previous chapter, NMR is a powerful tool for high-resolution structural analysis. However, serious drawbacks to this technique include the requirement for large amounts of material and the potential for macromolecules to possess poor solubility in solution. A typical 2D trNOESY experiment may take upwards of 24 hours to complete and demand a sample prepared to the highest degree of purity. An important complementary method for the characterization of protein structure and dynamics that alleviates many of the prerequisites for a successful NMR experiment is Matrix-Assisted Laser Desorption-Ionization Time-of-Flight Mass Spectrometry (MALDI-TOF MS). Whereas in NMR $\mu \mathrm{M}$ to $\mathrm{mM}$ concentrations of material are needed to collect a spectrum, MALDI-TOF MS proves to be a much more sensitive technique with detection limits reaching pM levels. MALDI-TOF MS tolerates small amounts of impurities, has short preparation/analysis times, and detects large macromolecules $(>300$ $\mathrm{kDa}$ ). In this chapter, the basics of the MALDI-TOF MS experiment will be detailed from the preparation of a sample to the acquisition of a spectrum, followed by a discussion of how hydrogen deuterium exchange (HDX) can be monitored with MALDITOF MS. 


\section{MALDI-TOF MS Theory and Instrumentation}

As the name implies, mass spectrometry detects molecules based on molecular mass. More precisely, the signal obtained reflects the mass to charge ratio $(\mathrm{m} / \mathrm{z})$ of the observed ion. A typical MS experiment is broken down into three parts: ion production, ion transmission, and ion detection (201). Figure 13 depicts a schematic of a Voyager DE (Delayed Extraction)-PRO MALDI-TOF MS instrument (Applied Biosystems). The following description pertaining to the theory and operation of the Voyager DE-PRO MALDI-TOF MS is adapted from the Voyager Biospectrometry Workstation User Guide provided by Applied Biosystems, unless referenced otherwise.

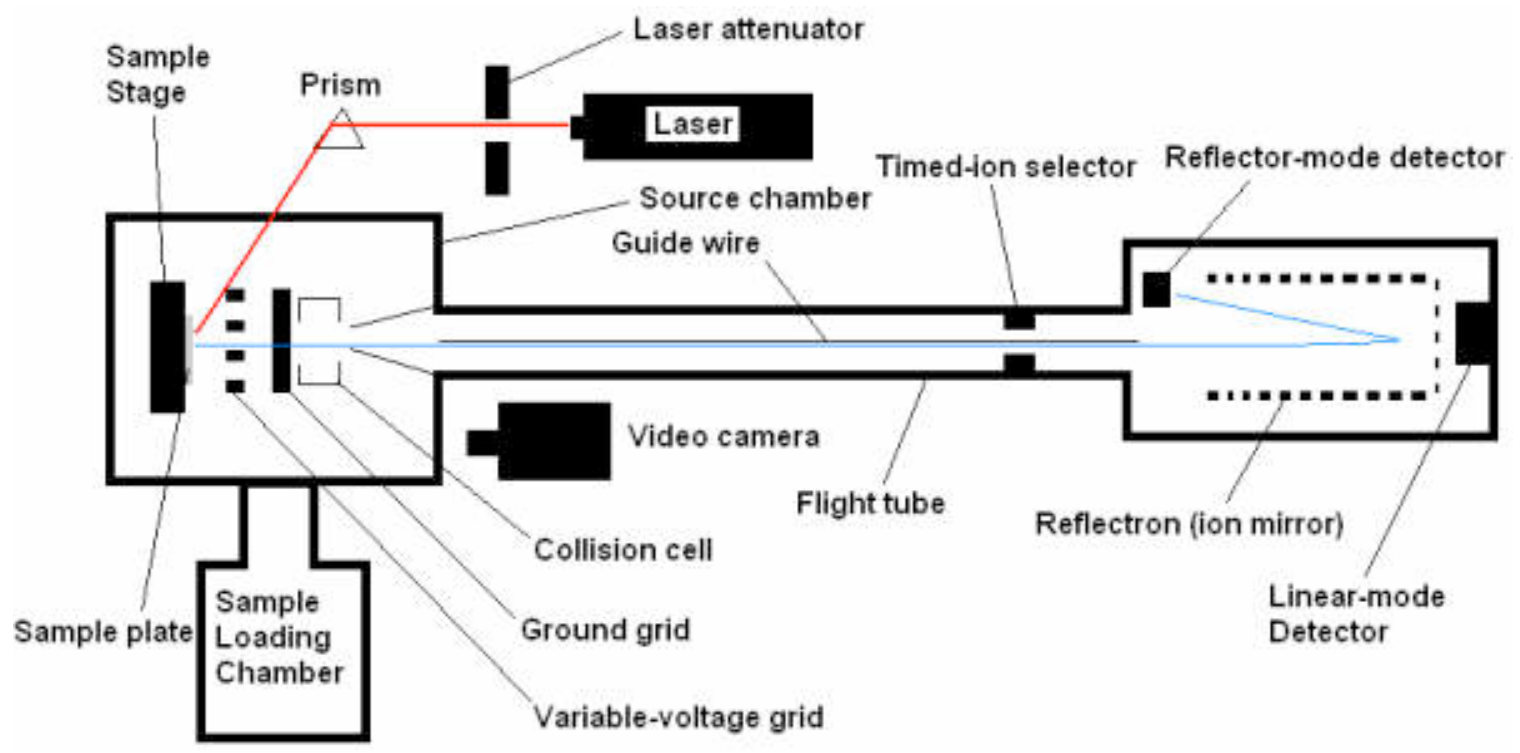

Figure 13: Schematic of a MALDI-TOF mass spectrometer. The red line is the path of the laser beam and the blue line is the path of the ions traveling in reflector mode. The figure is adapted from the Voyager Biospectrometry Workstation User Guide (Applied Biosystems).

Preparation of the sample is the first step in performing a MALDI-TOF MS experiment. Sample ionization proceeds with the assistance of the matrix. The choice of matrix depends on the composition of the material, i.e. protein, nucleic acid, or lipid, and the molecular weight of the substance studied. The Voyager DE PRO is equipped with a 
$\mathrm{N}_{2}$ laser with a wavelength of $337 \mathrm{~nm}$, therefore the matrix must absorb at $337 \mathrm{~nm}$. Table 2 presents a list of matrices most frequently employed in the projects described in this dissertation. These compounds are dissolved in a volatile, acidic solvent. A typical solution for $\alpha$-CHCA consists of 1:1:1 acetonitrile:0.1\% trifluoroacetic acid:ethanol at a concentration of $10 \mathrm{mg} / \mathrm{mL}$. Sample is mixed with matrix at a 1:1 ratio and allowed to dry on a stainless steel plate. After the matrix and sample co-crystallize, the plate is inserted into the mass spectrometer.

MALDI-TOF MS acquisition consists of the laser firing, the matrix absorbing the laser's energy, the sample ionizing, the ions traveling to the detector, and the instrument

\begin{tabular}{|c|c|c|}
\hline Table 2: Common Matrices Used with MALDI-TOF MS \\
\hline Matrix & Structure & Application \\
\hline $\begin{array}{c}\text { 3,5-Dimethoxy-4-hydroxy } \\
\text { cinnamic acid (Sinapinic }\end{array}$ \\
$\begin{array}{c}\text { Acid) } \\
\text { hydroxycinnamic acid } \\
\text { ( } \alpha \text {-CHCA) }\end{array}$
\end{tabular}


displaying the molecular mass of the ions in a spectrum (202). The laser fires coherent, high-density energy $(337 \mathrm{~nm})$ at a rate of $3 \mathrm{nsec}$ per pulse. An attenuator controls laser intensity, with a prism directing the beam to the sample plate. An excess of matrix over sample facilitates efficient sample ionization and desorption, as well as dissuades decomposition of the sample due to radiation, thus the process is termed "soft" ionization (203). It should be noted that ionization refers to the transfer of a proton from the matrix to the sample (204). Hence, analytes are recorded as $m / z$ values and not mass.

Once irradiated, a plume of gaseous material is ejected (desorption) from the surface of the sample plate $(204,205)$. Within this plume, a mixture of matrix, sample ions, and buffer components are in a dense cloud. The next step is to apply a potential $(20 \mathrm{kV})$ between the sample chamber and the variable voltage grid. Ions accelerate through the field-free flight tube, desirably with the same kinetic energy and in a straight path. Since $\mathrm{KE}=1 / 2 \mathrm{mv}^{2}$, smaller ions will reach the detector before larger species. A guide wire within the flight tube possesses a potential of $\sim 1 \%$ of the accelerating potential and serves to focus the ions into a more coherent path to the detector.

The system is maintained under a high vacuum $\left(10^{-6}-10^{-8}\right.$ torr $)$ for a few reasons. At atmospheric pressure, gaseous molecules will collide with the ions produced from desorption. Collisions of this nature result in the ions experiencing decreases in the kinetic energy and deviating from a straight path to the detector. These molecular interactions ultimately lead to errors in $\mathrm{m} / \mathrm{z}$ determinations. Another consideration is that constant bombardment of the ion detectors with molecules from the atmosphere will reduce sensitivity. Finally, the possibility exists for electrical arcing within the source chamber since the high voltages required for ion transmission to the detector could cause 
molecules within the air to momentarily conduct current. Therefore, after inserting the sample plate into the instrument, the sample chambers must be evacuated of nearly all gaseous molecules for proper instrument functioning and maintenance. Located at the opposite end of the instrument is the linear detector. The purpose of this device is to count the number of ions (intensity) that reach the detector and the time the ions arrive (drift time) at the detector. Signals recorded by the detector are sent to a digitizer for conversion to a mass spectrum displaying a plot of the intensity versus $\mathrm{m} / \mathrm{z}(206,207)$. The drift time $(t)$ is converted to $m / z$ using Equation 3, where $K E$ is kinetic energy, $s$ is drift distance, and $z$ is ionic charge.

$$
t=s\left(\frac{m}{2 * K E * z}\right)^{\frac{1}{2}}
$$

Equation 3 highlights the necessity of similar $K E$ and drift distance values for accurate $\mathrm{m} / \mathrm{z}$ determinations. An initial delay in the application of the accelerating potential is quite important for a few reasons (208). If the potential is not immediately applied, these plume components will drift apart and the initial ionization energy within the sample molecules will dissipate to the surroundings. Therefore, introduction of 20 $\mathrm{kV}$ to the system will be less likely to induce fragmentation within the less dense plume. Second, initial ionization will randomly impart varying degrees of energy to the ejected species. Acceleration of the ions immediately following ionization yields an uneven distribution of $K E$, skewing the detected $m / z$ values for the observed species (209). In order to avoid these issues, a delay of 100-200 nsec is implemented before engaging the accelerating potential (delayed extraction) $(210,211)$. This delay ensures that the ions will traverse the field free tube at the same time and with the same $K E$. 
Within the Voyager DE PRO, another type of detection involves ion reflection. Before reaching the linear detector, the ions are reflected by a single-stage gridded mirror (evenly spaced electrodes) that uses a uniform electric field to direct the ions to the reflector mode detector (212). In conjunction with delayed extraction, this process serves to minimize initial differences in $K E$ (213). Ions with higher $K E$ will penetrate deeper into the ion mirror, while ions with the same $\mathrm{m} / \mathrm{z}$ but lower $K E$ will not travel as far within the ion mirror. Thus, variations in $m / z$ due to $K E$ are minimized. Reflector mode significantly improves mass accuracy and signal resolution when compared to linear detection. The upper limit for reflector mode ion detection is $10,000 \mathrm{~m} / \mathrm{z}$. The reason for this threshold is related to peak broadening resulting from the metastable decay of higher MW ions (214). This type of broadening is greater than broadening associated with $K E$ variation, thus resolution is not enhanced in reflector mode.

\section{Applications of MALDI-TOF MS}

As previously described, MALDI-TOF MS primarily measures the mass of molecules. This technique has been utilized to observe conformational changes in protein dynamics that result from conversion of a zymogen to an active enzyme or of a ligand binding to a protein. The three principal methods for probing variations in protein dynamics with MALDI-TOF MS are chemical modification, limited proteolysis, and hydrogen deuterium exchange (HDX).

Differential chemical modification of specific amino acid residues was first implemented in our laboratory as a valuable tool for observing FXIII dynamics in 2004 (175). In this method, the protein is exposed to an amino acid modifying reagent, digested with a protease, and analyzed with MALDI-TOF MS. For example, unactivated 
and activated FXIII are both introduced to N-ethylmaleimide (NEM), which specifically modifies cysteine residues (215). FXIII possesses nine cysteines and once labeled, increases the $m / z$ value of the monitored digest fragment by $125.1 \mathrm{Da}$ (Figure 14). By

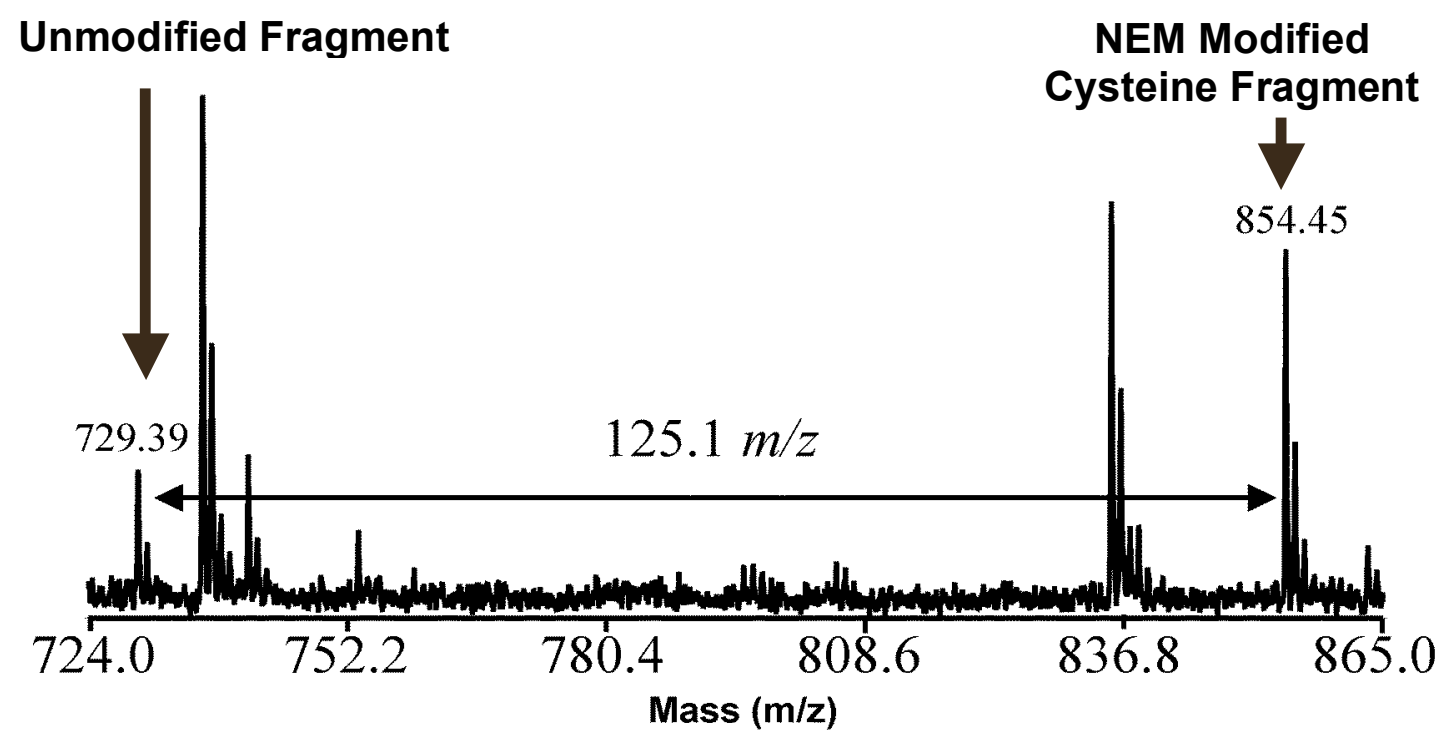

Figure 14: NEM modification of cysteine as detected by MALDI-TOF MS. FXIIIa is treated with NEM for 60 minutes, digested with trypsin, and analyzed with MALDI-TOF MS. The unmodified fragment represents $327-333(729.39 \mathrm{~m} / \mathrm{z}) . \quad \mathrm{C}^{327}$ is modified with NEM adding $125.1 \mathrm{~m} / \mathrm{z}$ to the tryptic peptide $(854.45 \mathrm{~m} / \mathrm{z})$

comparing the reaction of NEM with cysteine in zymogenic versus activated FXIII, information is obtained about the solvent accessibility of cysteine, which is then extrapolated to deduce conformational changes within the transglutaminase. Similarly, acetic anhydride reacts with available lysine residues providing complementary structural information $(216,217)$. An increase of $42 \mathrm{Da}$ is observed in the mass spectrum (Figure 15). Further detail concerning this methodology will be discussed in chapter VI.

Conformational changes can also be detected by proteolytically digesting the protein for only 30 minutes and recording the appearance and abundance of ions in the mass spectra (218). Such limited proteolysis provides a glimpse of peptide bond accessibility for the proteases chymotrypsin and trypsin. If one fragment appears in the 
digest of the zymogen and is absent in the digest of the activated form of the enzyme, then it is inferred that a conformational change has rendered this peptide bond less accessible to hydrolysis in the active form. Once again, the utility and implementation of this technique is presented in chapter VI.

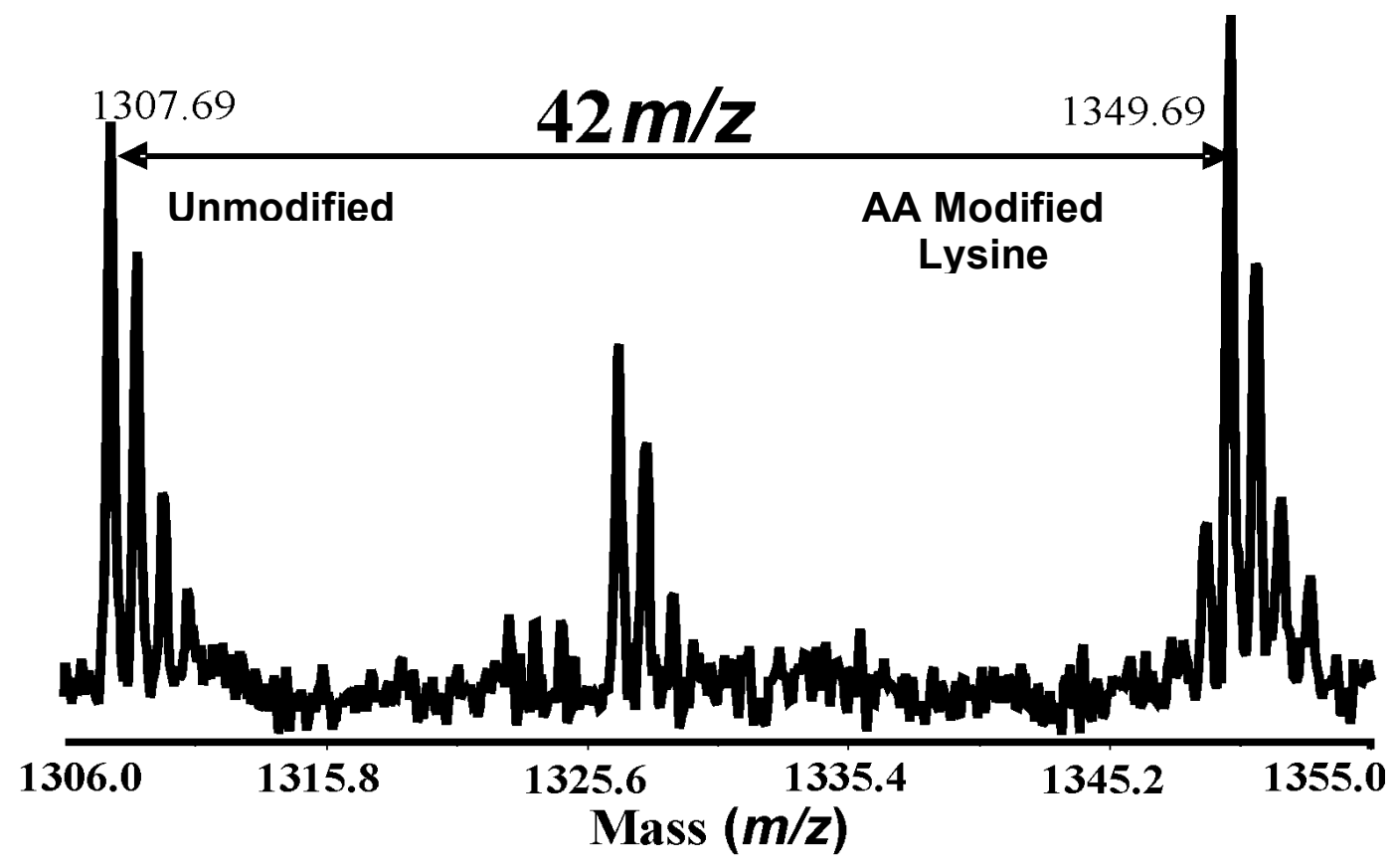

Figure 15: Acetic Anhydride modification of lysine as detected by MALDI-TOF MS. FXIIIa is treated with AA for 60 minutes, digested with trypsin, and analyzed with MALDI-TOF MS. The unmodified fragment represents $704-715(1307.69 \mathrm{~m} / \mathrm{z}) . \mathrm{K}^{704}$ is modified with AA adding $42 \mathrm{~m} / \mathrm{z}$ to the tryptic peptide $(1349.69 \mathrm{~m} / \mathrm{z})$

An important technique for observing perturbations in protein dynamics that does not rely on chemical modification of amino acids is hydrogen deuterium exchange (HDX). HDX within proteins has been studied for nearly a half a century (219). The method exploits differences in the rate of chemical exchange for backbone amide protons in proteins. Half-lives ranging from milliseconds to years is possible for amide protons in proteins due to secondary and tertiary structure, protein flexibility, and protein mobility (220-223). Equation 4 relates the rate of chemical exchange to the concentration 
of acid and base present in solution (224). Typical rate constants for polyalanine are $\mathrm{k}_{\mathrm{H}}=$

$$
\begin{gathered}
41.7 \mathrm{M}^{-1} \min ^{-1}, \mathrm{k}_{\mathrm{OH}}=1.12 \times 10^{10} \mathrm{M}^{-1} \min ^{-1} \text {, and } \mathrm{k}_{\mathrm{H} 2 \mathrm{O}}=3.16 \times 10^{-2} \mathrm{M}^{-1} \min ^{-1}(224) . \\
k_{e x}=k_{H}\left[H^{+}\right]+k_{\mathrm{OH}}\left[O H^{-}\right]+k_{\mathrm{H}_{2} \mathrm{O}}
\end{gathered}
$$

The rate of chemical exchange is highly dependent on $\mathrm{pH}$ and temperature. At a $\mathrm{pH}$ of 2.5 , the rate of proton exchange is at a minimum. More acidic conditions $(\mathrm{pH}<2.5)$ lead to catalysis occurring through proton addition and a more basic environment $(\mathrm{pH}>$ $2.5)$ promotes proton abstraction (220). In addition, exchange is much slower at $0{ }^{\circ} \mathrm{C}$ than at $25{ }^{\circ} \mathrm{C}$. Therefore, $\mathrm{HDX}$ quench conditions refer to dropping the $\mathrm{pH}$ to 2.5 at $0{ }^{\circ} \mathrm{C}$ where the exchange rate for backbone amide protons ranges from 30-120 minutes (225).

A few factors influence the rate of backbone amide proton exchange that are intrinsic to the protein (220-223). First, the degree of solvent accessibility for the proton is an important consideration. Surface-exposed amides are more available for deuterium exchange than protons buried within the interior of the protein. Hydrogen bonding, along with secondary and tertiary structural elements, involving the proton will provide stabilization against exchange, further slowing the rate of deuterium incorporation.

Protein dynamics influence deuterium exchange to a high degree (Figure 16) (226228). Through fluctuations in protein structure (temporary protein unfolding), a deeply buried proton may become briefly exposed to solvent. Therefore, the rate of protein unfolding $\left(\mathrm{k}_{\mathrm{u}}\right)$ and refolding $\left(\mathrm{k}_{\mathrm{f}}\right)$ will play a role in proton solvent accessibility. A smaller fluctuation event could be the momentary disruption of a H-bond leading to deuterium exchange. In this instance, the rate of opening $\left(\mathrm{k}_{\mathrm{op}}\right)$ and closing $\left(\mathrm{k}_{\mathrm{cl}}\right)$ of local protein structure need to be considered when following deuterium exchange (229). 

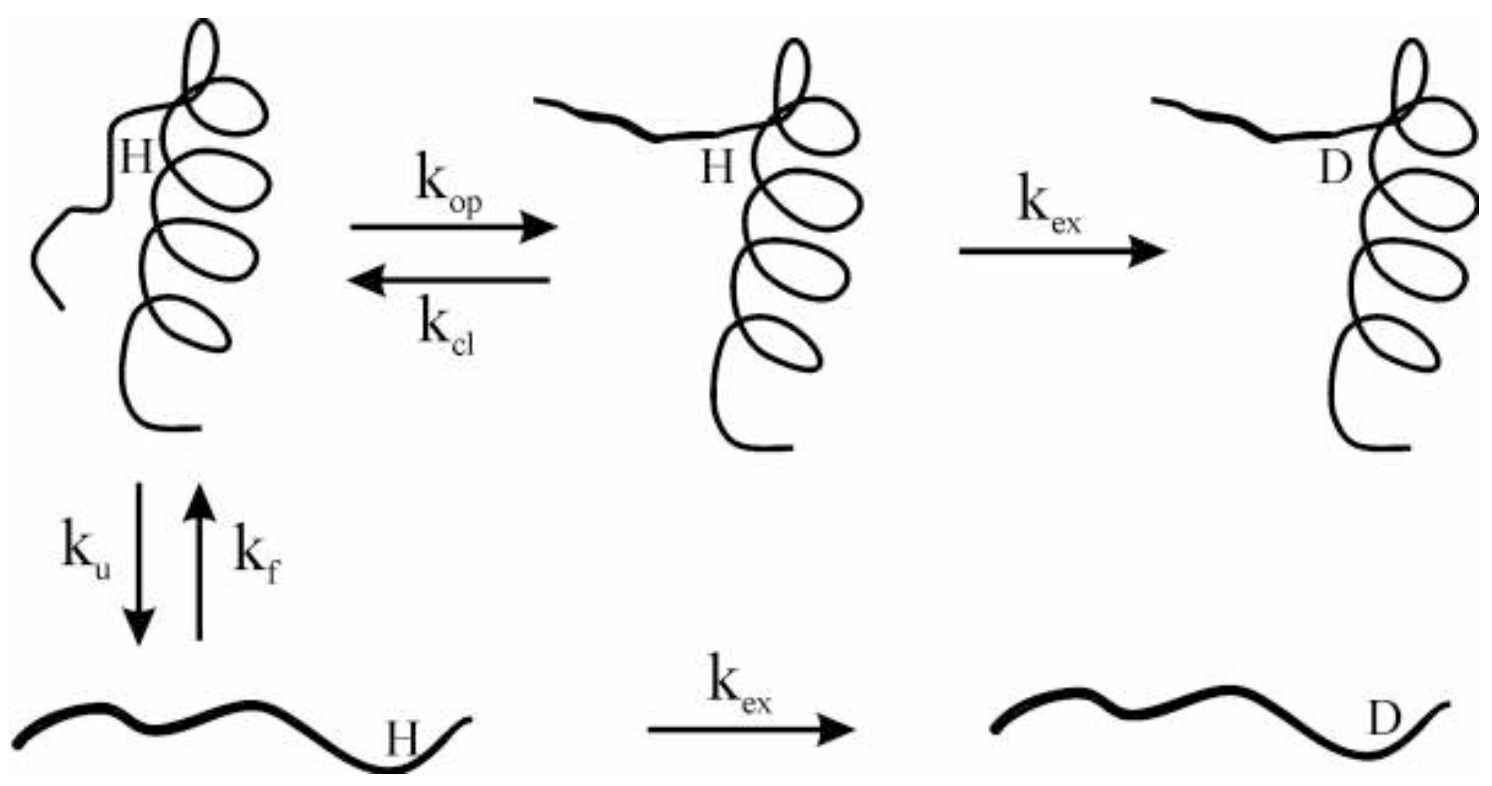

Figure 16: Pathways of protein backbone amide proton exposure (229). The top route illustrates a local unfolding event, whereas the bottom pathway depicts complete unfolding of the protein followed by deuterium exchange.

Two different types of deuterium exchange regimes describe the kinetic extremes of the HDX continuum $(221,228)$. In EX1 exchange, once the proton becomes solvent exposed, deuterium exchange immediately occurs. The observed rate of exchange is equal to the rate describing the opening of the particular structural element within the protein $\left(\mathrm{k}_{\mathrm{obs}}=\mathrm{k}_{\mathrm{op}}\right)$. The time for the protein to reversibly refold, or close, $\left(\mathrm{k}_{\mathrm{cl}}\right)$ is slower than deuterium exchange. At the other extreme, EX2 exchange refers to the situation where protein refolding after solvent exposure is faster than the rate of deuterium exchange. Thus, an equilibrium exists between the solvent accessible and properly folded protein states $\left(\mathrm{k}_{\mathrm{obs}}=\mathrm{k}_{\mathrm{op}} / \mathrm{k}_{\mathrm{cl}} * \mathrm{k}_{\mathrm{ex}}\right)$. Since the observed rate is proportional to the rate of exchange, the EX2 regime is sensitive to $\mathrm{pH}$ changes. Unless the rate of protein unfolding is dependent on $\mathrm{pH}$, the EX1 regime is independent of $\mathrm{pH}$. Most protons within a protein reside somewhere between the two exchange states. 
The Komives laboratory described the first applications of HDX with MALDI-TOF MS in 1998 (230, 231). Figure 17 illustrates the general protocol for the technique. Since all three projects described in this dissertation employ HDX methodology, the general protocol will be discussed in this chapter with the relevant features associated with each specific project detailed in the following chapters.

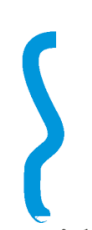

Peptide

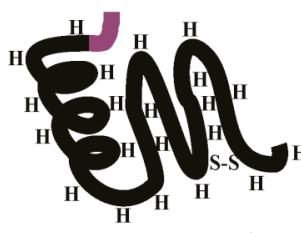

Dry Protein
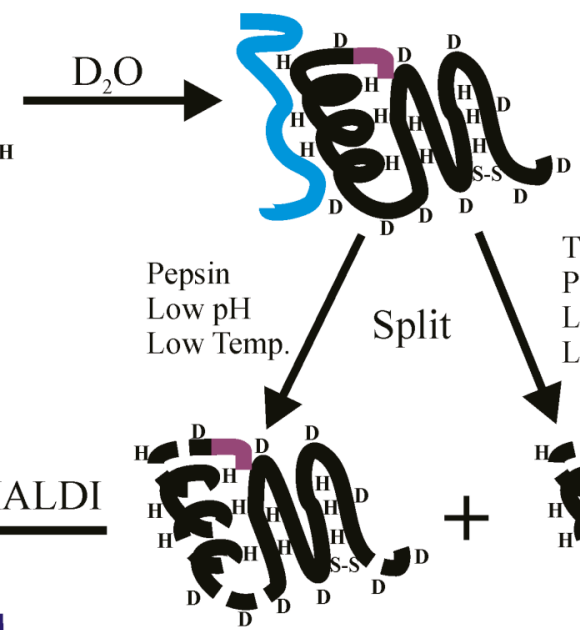

Peptic Fragments

Figure 17: Protocol for the hydrogen deuterium exchange experiment. In this schematic, a protein and peptide interact leading to protection from deuterium at the peptide-protein interface. After binding, a conformational change occurs at the purple fragment blocking exchange for several interior protons. The exchange is quenched by acidification and a decrease in temperature. Disulfides within a protein can be reduced with TCEP at the quench conditions. The protein is hydrolyzed with pepsin and then analyzed with MALDI-TOF MS.

HDX can be utilized to examine conformational changes associated with the conversion of a zymogen to an activated state. Additionally, the technique may be applied to study the consequences of ligand binding to a protein. The Komives laboratory was the first to implement HDX to study solvent accessibility at a proteinprotein interface (231). With such an HDX experiment, the ligand should be bound to the protein, ideally, for the entire course of the deuterium exchange $(173,231)$. Thus, the $\mathrm{K}_{\mathrm{D}}$ describing the strength of the ligand-protein interaction, as well as, the concentration 
of the binding partners, will determine the lifetime of the complex. In other words, the more time the ligand and protein spends apart directly affects protein deuterium incorporation at the binding interface. Equation 5 illustrates the equation that calculates the percentage of ligand $(L)$ bound to protein $(R)$ given the molar concentrations of both components and the $\mathrm{K}_{\mathrm{D}}$ are known $(173,231)$.

$$
\% \text { Bound }=\frac{L+R+K_{D} \pm \sqrt{\left(L+R+K_{D}\right)^{2}-4 L R}}{2 R}
$$

For a typical HDX experiment, the protein is exchanged into the desired buffer and lyophilized. Initiation of deuterium exchange occurs when the protein is reconstituted in $\mathrm{D}_{2} \mathrm{O}$. Next, the samples are incubated in a desiccator at room temperature for 1 minute or 10 minutes. The exchange is quenched by lowering the temperature to $0{ }^{\circ} \mathrm{C}$ and acidification to $\mathrm{pH}$ 2.5. Under the quench conditions, immobilized pepsin is introduced to the sample and digested for 10 minutes. Unlike the proteases chymotrypsin and trypsin, pepsin is most active at acidic $\mathrm{pH}$ conditions. Also, pepsin's specificity determinants differ from chymotrypsin and trypsin (232). Whereas chymotrypsin and trypsin hydrolyze peptide bonds after specific residues, pepsin relies on secondary and tertiary structural elements when cleaving proteins (223). Though predicting where pepsin cleaves proteins is a difficult prospect, proteolysis always yields the same fragments from digest to digest, even with similar proteins from different species (233).

At this point, it should be noted that others have employed a range of time points from 30 seconds to 10 minutes when studying $\operatorname{HDX}(172-174,230,231,233,234)$. The data were then fitted to an exponential equation that classifies protons as either fast or slow exchangers. The fitted equation yields values for the rate of proton exchange and 
the maximum amount of deuterium incorporation for the particular class of protons (fast or slow). Since the peptides examined in this HDX method consist of a multitude of backbone amide protons, assigning rate constants for individual amide protons is a difficult task. Furthermore, classifying protons as either fast or slow exchangers does not serve to advance a clearer understanding of the differences between protein states. Therefore, the HDX technique may be considered as more of a qualitative method to determine variations amongst several unique protein states. Observing differences in HDX for a protein at an initial time point (1 minute) and a longer time point (10 minutes) enables ligand binding interfaces to be deduced, as well as more transient deviations in HDX resulting from allostery due to ligand binding or activation of the protein.

Since thrombin possesses four disulfide bonds (51), both a reducing and nonreducing digest was necessary to produce maximal sequence coverage. Tris (2carboxyethyl) phosphine hydrochloride (TCEP $\mathrm{HCl}$ ) can efficiently reduce disulfide bonds in acidic conditions on ice (235) and does not interfere with pepsin hydrolysis of the protein $(167,234)$. Once pepsin hydrolysis has proceeded for 10 minutes, centrifugation at $4{ }^{\circ} \mathrm{C}$ separates the digest from the immobilized pepsin. Finally, $10 \mu \mathrm{L}$ aliquots are immediately frozen in liquid nitrogen and are stored at $-70{ }^{\circ} \mathrm{C}$.

MALDI-TOF MS analysis of the digested protein is the next step. A frozen HDX aliquot of the protein is thawed, immediately mixed with an equal volume of $10 \mathrm{mg} / \mathrm{mL}$ $\alpha$-CHCA matrix and then $0.5 \mu \mathrm{L}$ spotted onto a chilled stainless steel MALDI plate. The spotted MALDI plate is dried under a moderate vacuum and is inserted into the MALDITOF MS. The amount of time required for the plate to dry is roughly equivalent to the time needed for the MALDI-TOF MS to cycle through the eject protocol and accept the 
plate for analysis. The entire procedure takes about five minutes of time, limiting the amount of hydrogen back exchange.

The spectra are collected in the reflector mode with 256 laser shots per spectrum to maximize signal to noise ratios. Since pepsin's cleavage patterns are difficult to predict, peptide fragments within a mass spectrum require identification, primarily through post source decay (PSD) sequencing (236). Briefly, PSD is a technique that leads to peptide fragmentation within the MS through ionization with higher laser intensity and collisions with gas molecules introduced into the ion source. The resulting fragments are detected by the MS. By matching the $m / z$ values to theoretical fragments obtained from a known potential peptide sequence, identification of a particular peptide is achieved and differences in deuteration states can be localized to specific regions within the protein.

Thrombin peptides derived from a peptic digest without TCEP treatment were previously identified by Croy and co-workers (233). These assignments were verified in the current studies by in-house PSD sequencing. Peptides obtained from the reduction of IIa by TCEP were also identified in-house with additional assistance from Julia Koeppe (University of California at San Diego) $(167,234)$. Brian T. Turner, Jr. identified peptic fragments from the digest of FXIII (174). The program Data Explorer (Applied Biosystems) was used to analyze the spectra. Calibration of the spectra involved two reference peptides produced from the digest with pepsin.

Within the mass spectrum, compounds appear as an isotopic cluster. The first peak in the envelope represents the compound with only the dominant isotopes ${ }^{12} \mathrm{C},{ }^{16} \mathrm{O}$, and ${ }^{14} \mathrm{~N}$. Since the natural abundance of ${ }^{13} \mathrm{C}$ is $1.1 \%$ (237), the next peak in the cluster correlates to the probability of the compound possessing a single ${ }^{13} \mathrm{C}$. The third peak in 
the cluster is the compound with two ${ }^{13} \mathrm{C}$ atoms. The monoisotopic peak will dominate the cluster, until analyzing a compound with at least 100 carbon atoms, at which point the likelihood of the presence of a ${ }^{13} \mathrm{C}$ atom is greater than the molecule without ${ }^{13} \mathrm{C}$. The

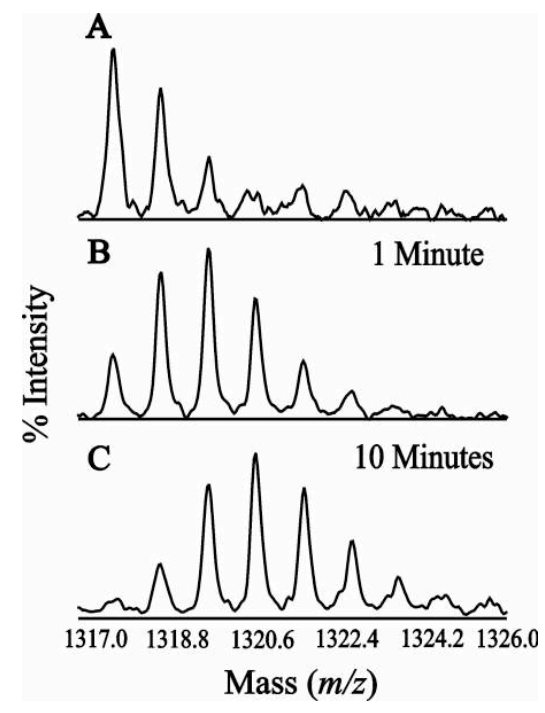

Figure 18: Isotopic envelope for a peptide after 0,1 , and 10 minutes of deuteration. Mass spectra representing residues $85-99$ of bovine thrombin (1317.73). A. Undeuterated fragment. B. One minute of deuteration. C. Ten minutes of deuteration. atoms oxygen and nitrogen also possess naturally occurring isotopes; however, ${ }^{18} \mathrm{O}(0.2 \%)$ and ${ }^{15} \mathrm{~N}$ (0.4\%) have lower abundances than ${ }^{13} \mathrm{C}(1.1 \%)(237)$. Since oxygen and nitrogen do not appear as frequently in peptides as carbon, these $\mathrm{O}$ and $\mathrm{N}$ isotopes will not contribute to the isotopic profile as visibly as ${ }^{13} \mathrm{C}$.

Introducing a peptide to deuterium will also serve to increase the $m / z$ by the amount of deuterium incorporated into the peptide. Deuterium is $1 \mathrm{Da}$ heavier than hydrogen, which is reflected in the shift of the isotopic cluster to the right. Figure 18 displays three time points from zero to ten minutes of

deuterium incorporation. As longer time points are observed, the peptide will incorporate more deuterium, eventually reaching a plateau.

Once the spectra are collected, the amount of deuterium incorporation must be calculated. Equation 6 has been developed to quantify the amount of deuterium uptake for each peptide observed in the spectra, where $I_{m}$ represents the average mass of the isotopic cluster (also termed centroid), $x_{i}$ is the intensity and $m_{i}$ is the mass of peak $i$. The summation encompasses all peaks in a cluster that have signal to noise ratios of at least two. By subtracting the undeuterated centroid from each deuterated centroid, the amount 
of deuterium incorporated into the peptide is quantified. At least three spectra were collected per trial and three trials were run for each time point under each condition.

$$
\mathbf{I}_{\mathrm{m}}=\sum_{i=1}^{n}\left(\frac{x_{i}}{\sum_{i=1}^{n} x_{i}}\right) m_{i}
$$

Quantification of differences in deuteration between different protein states was calculated with Equation 7, where $D$ is the amount of deuterium incorporated in the different protein state, $D_{\text {ref }}$ is the amount of deuterium incorporated for reference protein state, and $H_{e x}$ is the theoretical maximum number of exchangeable protons within the indicated peptide, assuming $100 \%$ deuteration. Only $\%$ differences more than $\pm 4.5 \%$ are considered significant $(174,238,239)$.

$$
\% \text { Difference }=\left(\frac{D-D_{\text {ref }}}{H_{e x}}\right) * 100 \%
$$

$H_{e x}$ can be divided into three categories. The first type is backbone NHs, calculated by subtracting the number of prolines and the N-terminal residue from the total amino acids in the peptide. The next two types rely on the final $\%$ of $\mathrm{D}_{2} \mathrm{O}$ in solution under quench conditions $(\sim 4.5 \%)$. The $\mathrm{N}$-terminus and C-terminus possess four exchangeable protons. Finally, 12 residues contain side chain protons that can exchange for deuterium: $\mathrm{R}(5), \mathrm{K}(3), \mathrm{H}(2), \mathrm{N}(2), \mathrm{Q}(2), \mathrm{C}(1), \mathrm{S}(1), \mathrm{T}(1), \mathrm{Y}(1), \mathrm{W}(1), \mathrm{E}(1)$, and $\mathrm{D}(1)$. The following is an example for calculating $H_{e x}$ for the IIa fragment 173-181 (RIRITDNMF). The total number of NHs is nine. From this, the N-terminal residue is subtracted, leaving 8 exchangeable backbone protons. At the termini reside four protons. To these protons are added the amount of exchangeable side chain protons: 2R (10 Hs), 1T $(1 \mathrm{H}), 1 \mathrm{D}(1$ 
$\mathrm{H})$, and $1 \mathrm{~N}(2 \mathrm{H})$. The termini plus side chain Hs equal 18, which is multiplied by the residual $\%$ of deuterium in solution under quench conditions: $18 \times 0.045=0.8$. Add 0.8 and 8 to arrive at $H_{e x}=8.8$ for $173-181$ of IIa.

Back exchange from deuterium to hydrogen resulting from humidity, temperature, quench solutions, and matrix accounted for a $50 \%$ loss of deuterium during analysis. This percentage was determined by performing the HDX protocol on a small model peptide. Experiments were performed within the same week to minimize HDX variations resulting from temperature and humidity fluctuations. In addition, a dehumidifier has been installed in the MALDI-TOF MS room. Thus, the amount of back exchange is essentially constant for each individual peptide, verified by the low standard deviation for three independent trials. Only differences in HDX between two states of the protein are desired, therefore a correction for deuterium loss was not necessary.

Mass spectrometry provides an important tool for studying protein structure. In the case of FXIII, MALDI-TOF MS probes conformational changes that result from activation and inhibition that otherwise would have gone undetected in the absence of the corresponding crystal structures. The difficulties in crystallizing FXIIIa are detailed in Chapter I. As for thrombin, HDX coupled with MALDI-TOF MS has identified sites of peptide interaction at ABE-II and variations in protein dynamics independent of the ligand interaction site. These variations in protein dynamics that occur due to peptideprotein interactions may go undetected in crystal structures. The relative ease in sample preparation, instrument operation, and data analysis further enhances the accessibility of this technique to researchers around the world. MALDI-TOF MS will continue to be an essential method in detailing protein structure and dynamics for years to come. 


\section{CHAPTER IV}

\section{CONFORMATIONAL ANALYSIS OF $\gamma^{\prime}$ PEPTIDE (410-427) INTERACTIONS WITH THROMBIN ANION BINDING EXOSITE-II}

\section{$\underline{\text { Introduction }}$}

The thrombin anion binding exosites are highly electropositive patches that flank the catalytic triad of this serine protease (Figure 4B) (51). Binding to these exosites can modulate thrombin specificity or act in the recruitment of inhibitors $(34,240)$. At the time this research was undertaken, a crystal structure illustrating $\gamma^{\prime}$ peptide binding to thrombin's ABE-II had not been solved. As a result, the conformation the peptide adopted upon binding to thrombin and the exact ligand interface on the surface of thrombin were unknown.

$\mathrm{X}$-ray structures of ligands interacting with ABE-II have been reported in the literature $(66,96,97,168,169)$. Figure 19 displays the residues that form ABE-II and some of ligands bound to ABE-II (it should be noted that the views of IIa are different than Figure 4. The structures are flipped $90^{\circ}$ on the z-axis and rotated $180^{\circ}$ relative to the horizontal plane for a head on view of ABE-II). The structures illustrate two unique modes of ABE-II interactions. In the first set of structures, prothrombin fragment II (169) and haemadin (66) are illustrated reaching toward $\mathrm{R}^{97}, \mathrm{R}^{173}$, and $\mathrm{R}^{175}$. Both of these proteins utilize secondary structural elements while binding to IIa. Alternatively, GpIb $\alpha(96,97)$ and heparin (168) bind to a groove formed by $\mathrm{R}^{93}, \mathrm{R}^{101}, \mathrm{~K}^{236}$, and $\mathrm{K}^{240}$ in 
an extended conformation. Due to the high degree of sequence similarity to GpIba, perhaps the $\gamma^{\prime}$ peptide binds to ABE-II in a similar fashion (Table 1).

Differing perspectives exist regarding the transmission of allostery resulting from ABE-II binding. Though allosteric communication from ABE-II to the active site is a fairly well accepted concept $(67,68,113)$, more ambiguous is the linkage amongst ABE-I and $\mathrm{ABE}-\mathrm{II}(70,170,171)$. Hydrogen deuterium exchange (HDX) experiments provide a method to observe the regions of thrombin influenced by ligand binding and enhance the understanding of how allosteric changes are transmitted from ABE-II throughout the protease. Previous HDX work with the ABE-I binding protein thrombomodulin demonstrated that deuterium exchange for several regions within thrombin is affected, suggesting allostery from ABE-I to $\mathrm{ABE}-\mathrm{II}(172,173)$. With $\gamma^{\prime}$ peptide binding to ABEII, maybe HDX could detect a reverse pathway from ABE-II to ABE-I.

The research described in this chapter examines the $\gamma^{\prime}$ peptide's interaction with IIa's ABE-II. 1D and 2D NMR experiments were carried out to observe, in solution, the structural features the $\gamma^{\prime}$ peptide adopts upon interacting with IIa's ABE-II. Furthermore, the individual residues within the $\gamma^{\prime}$ peptide that contact the enzyme's surface could be detected. In another series of studies, HDX monitored by matrix-assisted laser desorption time-of-flight mass spectrometry (MALDI-TOF MS) was used to characterize the effects of $\gamma^{\prime}$ peptide binding on the solvent accessibility of IIa's surface. Additional coverage of IIa was obtained through the use of the reducing agent TCEP, which enabled the monitoring of previously unseen regions of thrombin. In these NMR and MS studies, phosphotyrosines, which do not sacrifice binding affinity, were prepared instead of the acid labile sulfotyrosines present in the fibrinogen $\gamma^{\prime}$ chains $(69,241)$. 

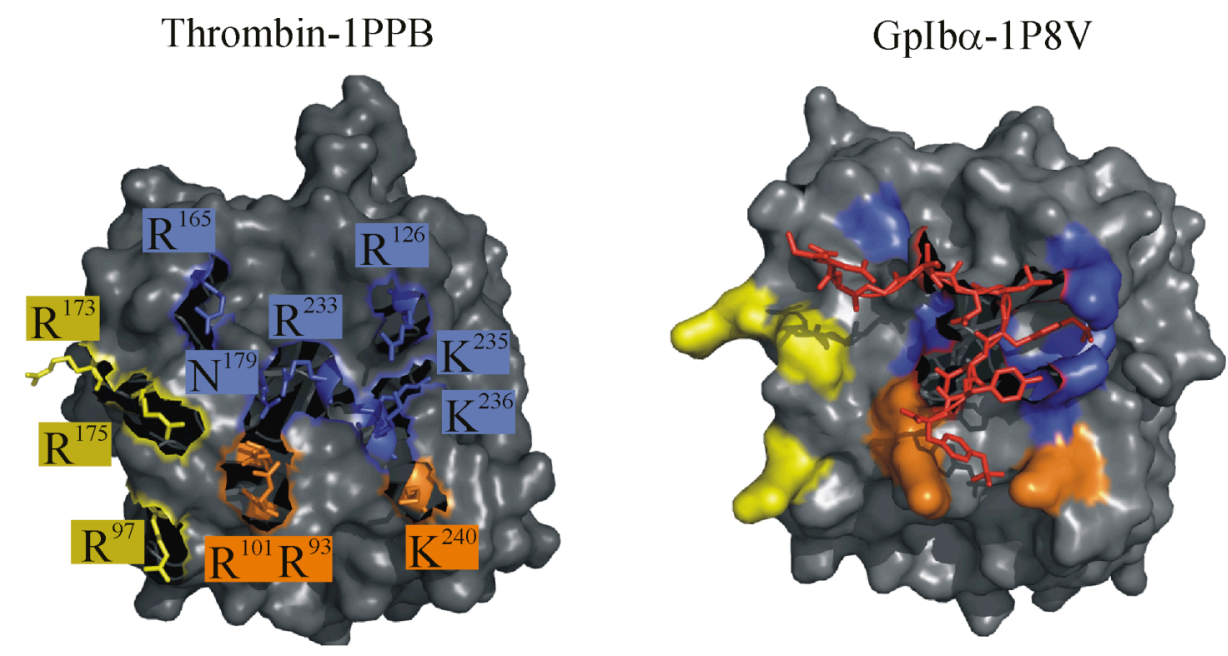

Prothrombin Fragment 2-1HPQ
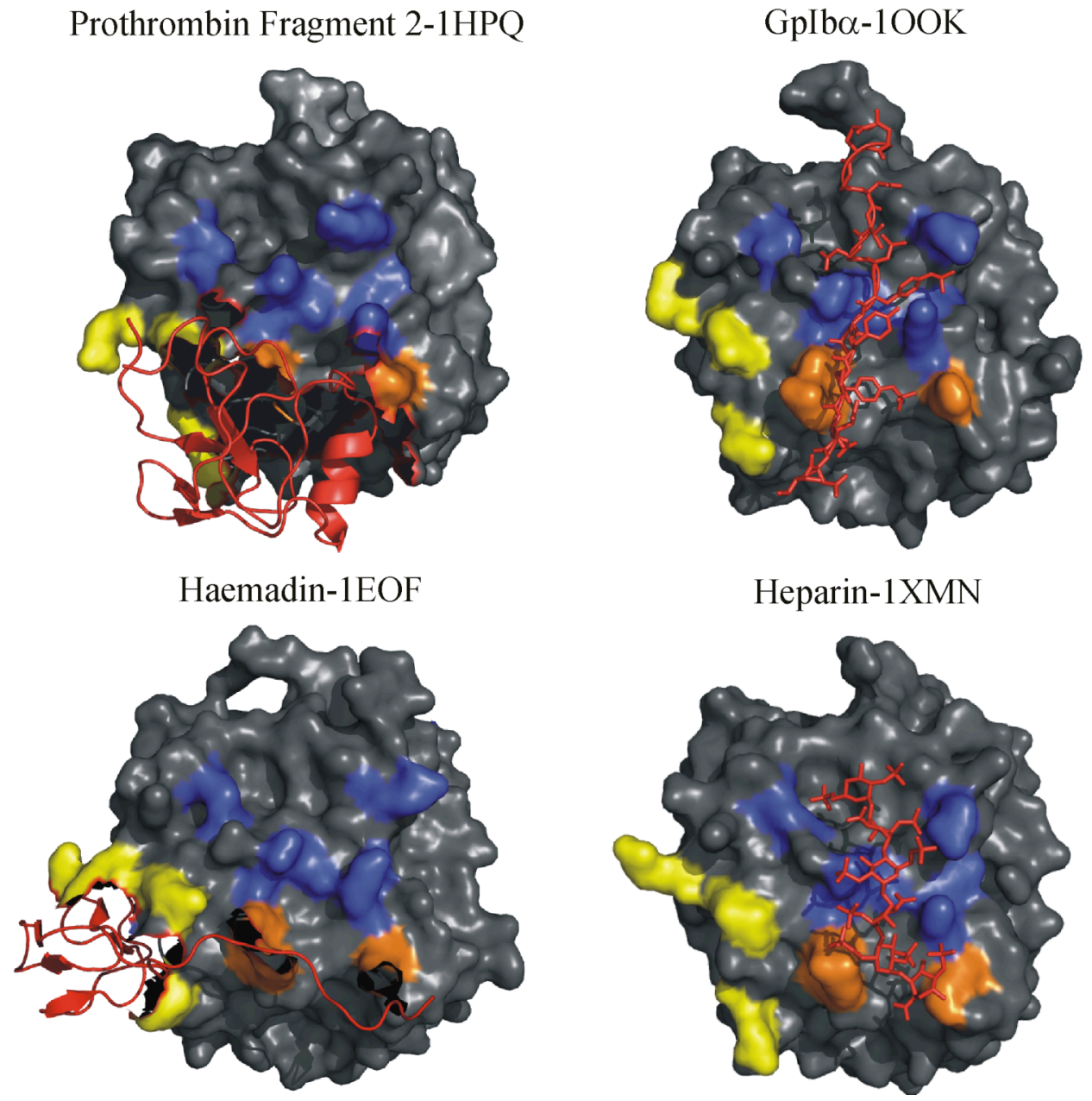

Figure 19: Modes of ligand binding to ABE-II. In the 1PPB structure, key ABE-II residues are labeled and color-coded. The orange residues interact with all ligands depicted in this figure. The blue residues form a groove that is utilized by heparin and GpIba. The polypeptides prothrombin fragment 2 and haemadin reach toward the yellow residues, skirting the ABE-II binding groove formed by the blue residues. 
Our findings describe the $\gamma^{\prime}$ peptide-thrombin relationship from the perspective of both the peptide and the enzyme. $\gamma^{\prime}$ peptide binding to ABE-II causes the ligand to adopt significant secondary structure when compared to free peptide in solution. Interactions at ABE-II also have allosteric consequences on the rate of deuterium exchange for regions unaffiliated with the ligand-protease interface. Fresh analysis of the results obtained in this investigation (167) will be compared to the crystal structure recently published by the Di Cera lab (242).

\section{Materials and Methods}

Synthetic Peptides. SynPep (Dublin, Ca) and Research Genetics, Inc (Huntsville, Al) synthesized peptides based on residues 410-427 (PEHPAETEYDSLYPEDDL) of the human fibrinogen $\gamma^{\prime}$ chain. Four variants of this sequence were prepared (Table 3). MALDI-TOF measurements on an Applied Biosystems Voyager DE-Pro mass spectrometer were used to verify the peptide $\mathrm{m} / \mathrm{z}$ values. The concentrations of the peptides in solution were determined by quantitative amino acid analysis (AAA Service Laboratory, Boring, OR).

\begin{tabular}{|c|c|}
\hline $\mathbf{Y}_{\mathbf{P}}^{418} \mathbf{Y}_{\mathbf{P}}^{422}$ & ${ }^{410}$ PEHPAETE $_{\mathbf{P}}$ DSLY $\mathbf{Y}_{\mathbf{P}}$ PEDDL $^{42}$ \\
\hline $\mathbf{Y}_{\mathbf{P}}^{418} \mathbf{Y}^{422}$ & ${ }^{410}{ }^{\text {PEHPAETE }} \mathbf{Y}_{\mathbf{P}}$ DSLYPEDDL ${ }^{427}$ \\
\hline $\mathrm{Y}^{418} \mathbf{Y}_{\mathbf{P}}^{422}$ & 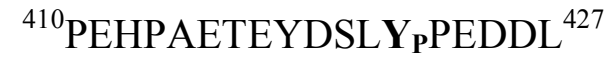 \\
\hline $\mathrm{Y}^{418} \mathrm{Y}^{422}$ & ${ }^{410}$ PEHPAETEYDSLYPEDDL ${ }^{427}$ \\
\hline
\end{tabular}

Thrombin Preparation. The isolation and purification of thrombin from bovine plasma barium sulfate eluate (BSE) (Sigma) was performed as previously described by Trumbo and Maurer (243). The purified thrombin solution was concentrated with Amicon Ultra-15 centrifugal filter units (Millipore) in an Allegra 21R Centrifuge 
(Beckman Coulter) run at $4000 \mathrm{rpm}$ and $4{ }^{\circ} \mathrm{C}$. The concentration of the activated thrombin was determined using the extinction coefficient of $\mathrm{E}^{1 \%}=19.5$ (244), then aliquoted and frozen at $-70{ }^{\circ} \mathrm{C}$ for future use.

Bovine IIa was used as the enzyme during the course of this work, while peptides derived from the fibrinogen $\gamma^{\prime}$ chain were based on the human sequence. Human and bovine IIa exhibit a high degree of sequence conservation (87\% identical) (51). Important regions where there are no differences include the active site, the $\mathrm{Na}^{+}$binding site, the $\beta$-insertion (Trp ${ }^{60 \mathrm{D}}$ ) loop, ABE-I, and ABE-II. As for the $\gamma^{\prime}$ chain, the bovine $\gamma^{\prime}$ sequence terminates at residue 419 (111), while the human sequence possesses an additional eight residues $(60,61)$. In this case, a peptide derived from the bovine $\gamma^{\prime}$ chain may not accurately portray human $\gamma^{\prime}$ chain binding to IIa. The interaction of the human $\gamma^{\prime}$ peptide with bovine IIa should not be influenced by the minor differences between the species. Supporting this statement is an HDX study, where PPACK inhibition of human and bovine IIa affected HDX in a similar manner for analogous peptides from both types of IIa (233). In addition, 2D trNOESY NMR examining the structural features adopted by fibrinopeptide A $(193,196,245,246)$ and a PAR1 peptide (185) bound to bovine IIa were in agreement with the corresponding human IIa X-ray structures $(247,248)$.

${ }^{1} H$ and ${ }^{31} P$ NMR Sample Preparation and Analysis. Approximately a 1:10 ratio of IIa to peptide was maintained for each complex studied. Sample preparation and analysis procedures similar to those of Trumbo and Maurer were employed (190). Selective features are summarized here. Each $400 \mu \mathrm{l}$ NMR sample contained $1.5 \mathrm{mM}$ peptide and either zero or 151-162 $\mu \mathrm{M}$ IIa. The samples were buffered in $25 \mathrm{mM} \mathrm{H}_{3} \mathrm{PO}_{4}, 150 \mathrm{mM}$ $\mathrm{NaCl}, 0.2 \mathrm{mM}$ EDTA, pH 5.6 and 10\% $\mathrm{D}_{2} \mathrm{O}$ (NMR buffer). Human gamma-IIa 
(Haematologic Technologies, Inc.) at $56 \mu \mathrm{M}$ was buffer exchanged into the NMR buffer and concentrated to $153 \mu \mathrm{M}$ via ultracentrifugation. With addition of the $\mathrm{Y}_{\mathrm{P}}{ }^{418} \mathrm{Y}_{\mathrm{P}}{ }^{422}$ peptide, the final ratio of gamma-IIa to peptide was $11: 1$ in $10 \% \mathrm{D}_{2} \mathrm{O}$.

All ${ }^{1} \mathrm{H}$ NMR experiments were performed on either a Varian Inova $800 \mathrm{MHz}$ spectrometer equipped with a triple resonance probe and pulsed-field triple axis gradients or a Varian Inova $500 \mathrm{MHz}$ equipped with a triple resonance probe and pulsed-field zaxis gradients. 2D transferred NOESY experiments of the enzyme-peptide complexes were run at 15 or $17{ }^{\circ} \mathrm{C}$ with 32 transients, $512 t_{1}$ increments, a mixing time of $400 \mathrm{~ms}$, and a spectral width of $8006 \mathrm{~Hz}$ or $5006 \mathrm{~Hz}$. Spectra were processed using FELIX2000 software (Accelerys, San Diego, CA) on a Silicon Graphics Octane workstation. For ${ }^{31} \mathrm{P}$ NMR, spectra were recorded on a Varian Inova $500 \mathrm{MHz}$ equipped with a $5 \mathrm{~mm}$ broadband probe tuned to $202.4 \mathrm{MHz}$. The spectra were referenced to the peak corresponding to $\mathrm{H}_{3} \mathrm{PO}_{4}(0.00 \mathrm{ppm})$.

HDX Sample Preparation. Aliquots of $100 \mu \mathrm{L}, 142-150 \mu \mathrm{M}$ IIa were buffer exchanged with Amicon-Ultra 4 units (10,000 MWCO) and diluted into $75 \mathrm{mM} \mathrm{NaCl}$, $12.5 \mathrm{mM} \mathrm{NaH}_{2} \mathrm{PO}_{4}, \mathrm{pH} 6.5$ (HDX Buffer). The final target concentration of IIa for all HDX preparations was from 20-25 $\mu \mathrm{M}$. D-Phe-Pro-Arg-chloromethyl ketone (PPACK) inhibited IIa was prepared by adding $5 \mu \mathrm{L}$ of $11 \mathrm{mM}$ PPACK to a thawed IIa sample diluted to $545 \mu \mathrm{L}$ with the HDX buffer (resulting in a 4:1 ratio of PPACK to IIa). The PPACK-IIa solution was incubated at $37^{\circ} \mathrm{C}$ for at least 30 minutes. An assay involving the chromogenic substrate S2238 was employed to establish that IIa was inactivated by PPACK. Following inactivation, PPACK-IIa was buffer exchanged in the same manner as active IIa. For samples containing the $\gamma^{\prime}$ peptide, $25 \mu \mathrm{L}$ of $480 \mu \mathrm{M} \gamma^{\prime}$ peptide in dI 
$\mathrm{H}_{2} \mathrm{O}$ was added to $24 \mu \mathrm{L}$ of the buffer exchanged IIa $(20-25 \mu \mathrm{M})$. The aliquots of the buffer-exchanged IIa, with and without the peptide, were evaporated to dryness using a SpeedVac unit (Savant). The dry aliquots were stored at $-70^{\circ} \mathrm{C}$.

HDX Experiments. Dry aliquots of IIa or $\mathrm{Y}_{\mathrm{P}}^{418} \mathrm{Y}_{\mathrm{P}}^{422}$ peptide-IIa were allowed to come to room temperature before beginning the experiment. $12 \mu \mathrm{L}$ of $99.996 \% \mathrm{D}_{2} \mathrm{O}$ (Cambridge Isotope Laboratories) were added to the aliquot yielding the final concentrations: $40-50 \mu \mathrm{M} \mathrm{IIa/PPACK-IIa,} \mathrm{with} \mathrm{or} \mathrm{without} 1000 \mu \mathrm{M} \gamma^{\prime}$ peptide, in 150 $\mathrm{mM} \mathrm{NaCl}, 25 \mathrm{mM} \mathrm{NaH}_{2} \mathrm{PO}_{4}, \mathrm{pH}$ 6.5. The samples were incubated in a desiccator at room temperature for 1 minute or 10 minutes. An initial set of HDX experiments were performed with varying concentrations of the $\gamma^{\prime}$ peptide $(150-1500 \mu \mathrm{M})$ to determine the target peptide concentration and to address potential non-specific binding interactions.

Since IIa possesses four disulfide bonds, both a reducing and non-reducing digest was necessary to produce maximal sequence coverage. Adding $114 \mu \mathrm{L}$ of $0.1 \%$ trifluoroacetic acid (TFA) (on ice) acted to acidify ( $\mathrm{pH} 2.5$ ) the solution and quench HDX. Immediately, $66 \mu \mathrm{L}$ was transferred to a tube of activated pepsin bound to $6 \%$ agarose (Pierce Chemical). To the remaining quenched IIa, $6 \mu \mathrm{L}$ of $1 \mathrm{M}$ TCEP (Tris (2carboxyethyl) phosphine hydrochloride, Pierce Chemical) was introduced, quickly vortexed, and the entire solution added to another tube of activated pepsin. After deuteration was quenched, the entire process of splitting IIa into a non-reducing and reducing pepsin digest took 30-40 seconds. Digestion occurred on ice for 10 minutes. Centrifugation at $4{ }^{\circ} \mathrm{C}$ separated the digest from the pepsin and $10 \mu \mathrm{L}$ aliquots were immediately frozen in liquid nitrogen and stored at $-70{ }^{\circ} \mathrm{C}$. 
HDX Analysis. A frozen HDX aliquot of IIa, with or without peptide, was thawed, immediately mixed with an equal volume of $10 \mathrm{mg} / \mathrm{mL} \alpha$-cyano-hydroxycinnamic acid matrix (Aldrich) in 1:1:1 ethanol/acetonitrile/0.1\% TFA, $\mathrm{pH} 2.2$, and then $0.5 \mu \mathrm{L}$ spotted onto a chilled stainless steel MALDI plate. The spotted MALDI plate was dried within a SpeedVac unit (Savant) and inserted into the MALDI-TOF MS. The amount of time required for the plate to dry was roughly equivalent to the time needed for the MALDITOF MS to cycle through the eject protocol and accept the plate for analysis. The entire procedure took about five minutes of time, limiting the amount of hydrogen back exchange. The spectra were collected in the reflector mode over a mass range of 800$3500 \mathrm{~m} / \mathrm{z}$ with 256 laser shots per spectrum. Peptides obtained from the peptic digest of IIa were previously identified. New coverage obtained by reducing the disulfides in IIa with TCEP was identified in-house and independently verified.

The program Data Explorer (Applied Biosystems) was used to analyze the spectra. Calibration of the spectra involved two reference peptides, one with the singly protonated monoisotopic mass of $888.4943 \mathrm{Da}$ (residues 46-52) and the other with the singly protonated pentaisotopic mass of $2106.1505 \mathrm{Da}$ (residues 85-99). The amount of deuterium uptake by each peptide and the $\%$ difference in deuteration relative to IIa was quantified as described by Sabo et al. (167) and discussed in Chapter III.

\section{$\underline{\text { Results }}$}

One-Dimensional Line Broadening NMR. One-dimensional proton NMR spectra of the different fibrinogen $\gamma^{\prime}$ peptides were obtained in the absence and presence of IIa. The appearance of resonance broadening in the spectra is indicative of peptide protons coming in direct contact with the IIa surface. Figure 20 illustrates the extensive binding 
interactions observed for the $\mathrm{Y}_{\mathrm{P}}^{418} \mathrm{Y}_{\mathrm{P}}^{422}$ peptide. Widespread line broadening is observed for the entire 1D spectrum, including the NHs spanning residues $\mathrm{H}^{412}$ to $\mathrm{L}^{427}$, the $\beta, \delta$, and $\varepsilon$ protons of $\mathrm{Y}_{\mathrm{P}}^{418}$ and $\mathrm{Y}_{\mathrm{P}}^{422}$, the $\beta$ protons of $\mathrm{D}^{419}, \mathrm{D}^{425}$, and $\mathrm{D}^{426}$, and the $\beta, \gamma$, and $\delta$ protons of residues $\mathrm{L}^{421}$ and $\mathrm{L}^{427}$. Only three observable proton resonances in Figure 20 remain unchanged with the addition of the enzyme: $\mathrm{P}^{410} \beta, \mathrm{E}^{411} \mathrm{NH}$, and $\mathrm{H}^{412} \delta$.
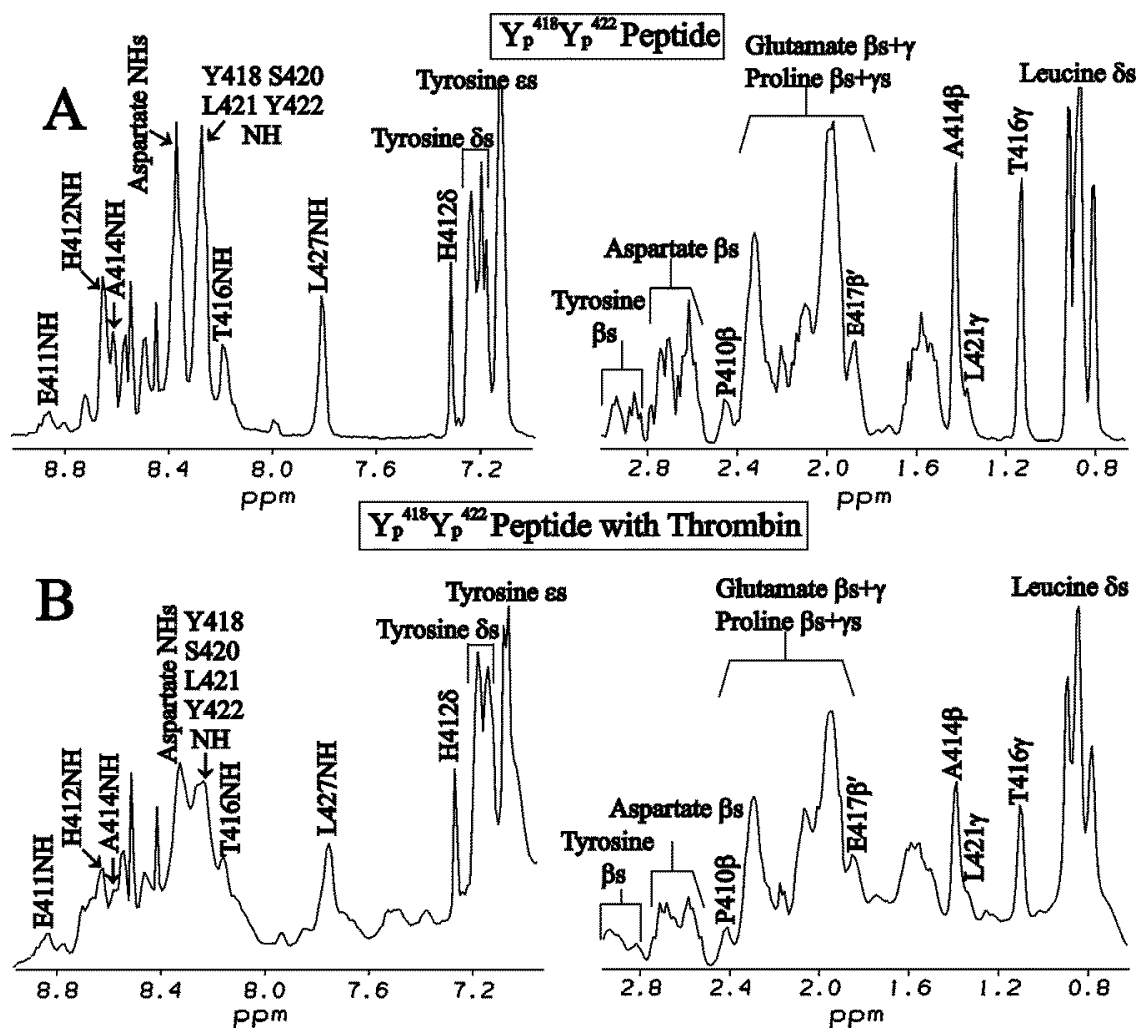

Figure 20: Line broadening for the $\gamma^{\prime}\left(\mathrm{Y}_{\mathrm{P}}{ }^{418} \mathrm{Y}_{\mathrm{P}}{ }^{422}\right)$ peptide in the presence of thrombin. A. 1D NMR spectrum for $1.5 \mathrm{mM}$ peptide in solution. B. 1D NMR spectrum for $1.5 \mathrm{mM}$ peptide in the presence of $0.162 \mathrm{mM}$ thrombin. All NMR samples are in $25 \mathrm{mM} \mathrm{H}_{3} \mathrm{PO}_{4}, 150 \mathrm{mM} \mathrm{NaCl}, 200 \mu \mathrm{M}$ EDTA, and $10 \%$ $\mathrm{D}_{2} \mathrm{O}, \mathrm{pH} 5.6$ (NMR Buffer). All observable protons make extensive contact with the surface of thrombin except $\mathrm{P}^{410} \beta, \mathrm{E}^{411} \mathrm{NH}$, and $\mathrm{H}^{412} \delta$.

To assess the contributions that each $Y_{P}$ makes to the interaction with IIa, singly phosphorylated peptides were also analyzed. The spectrum for the $\mathrm{Y}_{\mathrm{P}}^{422}$ peptide indicates broadened peaks for several residues (Figure 21). These spectra provide evidence that phosphorylation at a single $\mathrm{Y}$ is sufficient to promote binding of the $\gamma^{\prime}$ 
peptide to IIa. The absence of the phosphate at $\mathrm{Y}^{418}$, however, abrogates enzyme contact for at the least the protons: $\mathrm{H}^{412} \mathrm{NH}, \mathrm{A}^{414} \mathrm{NH}$ and $\beta, \mathrm{T}^{416} \gamma, \mathrm{Y}^{418} \varepsilon$, and $\mathrm{L}^{421} \gamma$ and $\delta$ s.

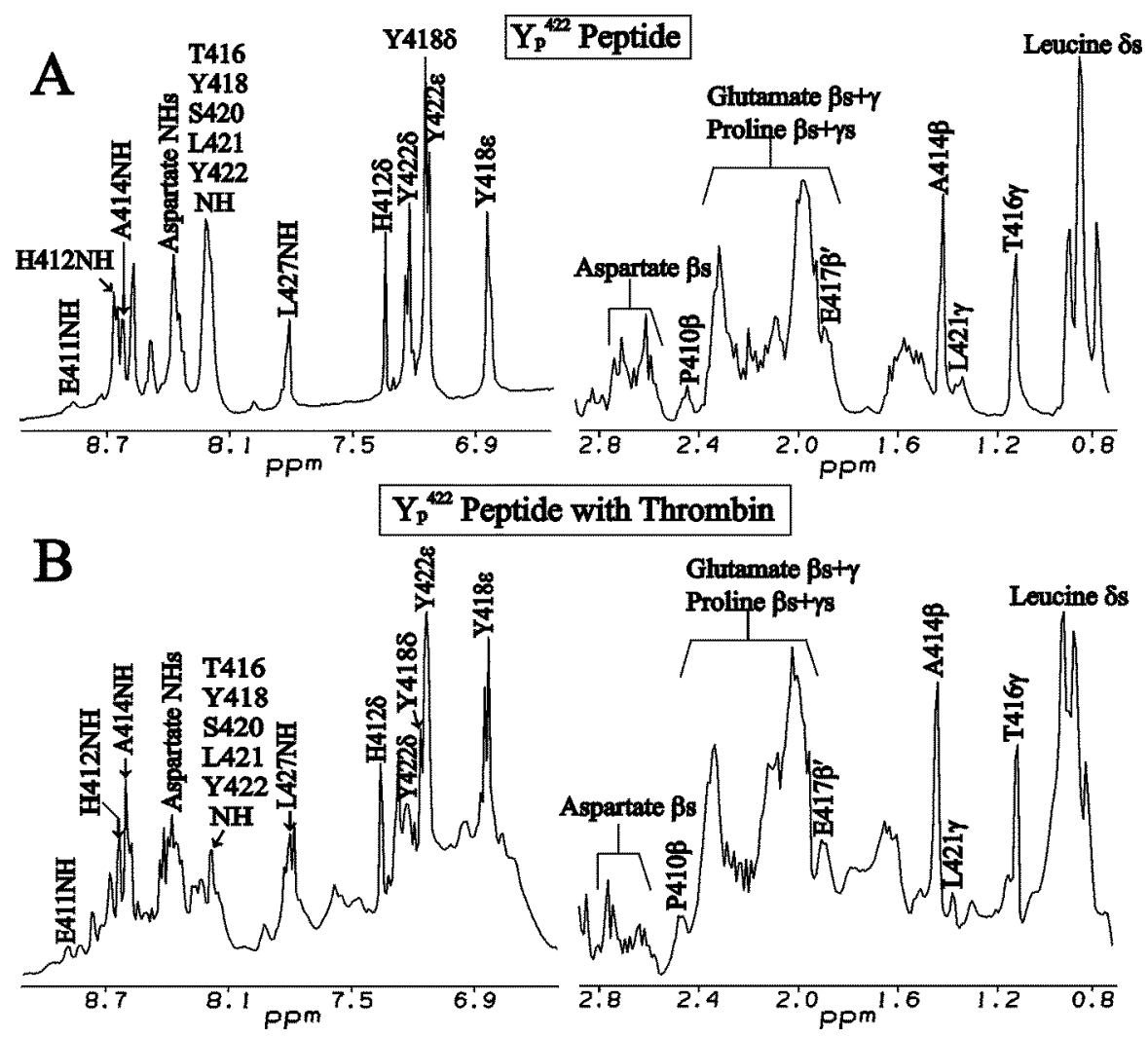

Figure 21: Line broadening for the $\gamma^{\prime}\left(\mathrm{Y}_{\mathrm{P}}^{422}\right)$ peptide in the presence of thrombin. A. 1D NMR spectrum for $1.5 \mathrm{mM}$ peptide in solution. B. 1D NMR spectrum for $1.2 \mathrm{mM}$ peptide in the presence of $0.122 \mathrm{mM}$ thrombin. The experimental conditions are the same as in Figure 27. In contrast to the previous figure, several residues experience a decrease in line broadening including the observable protons of $\mathrm{H}^{412}, \mathrm{~A}^{414}, \mathrm{~T}^{416}, \mathrm{Y}^{418}$, and $\mathrm{L}^{421}$.

With the $\mathrm{Y}_{\mathrm{P}}^{418}$ peptide, the appearance of much weaker enzyme binding is evident when examining the spectra in Figure 22, suggesting that most protons are not contacting the surface of the enzyme. The only significant amount of line broadening that can unambiguously be assigned is observed for the following protons: $A^{414} \beta, E^{417} \mathrm{NH}$ and $\beta^{\prime}, \mathrm{Y}^{418} \delta$ and $\varepsilon$, and $\mathrm{D}^{419}, \mathrm{D}^{425}$, and $\mathrm{D}^{426} \beta$ s. The amide region of the $\mathrm{Y}_{\mathrm{P}}^{418}$ spectra also displays broadening for a peak that includes the $\mathrm{NH}$ of $\mathrm{H}^{412}, \mathrm{~A}^{414}$, and $\mathrm{E}^{424}$, indicating that all three of these amide protons are contacting the surface of thrombin. To complete 
the series, 1D spectra of the unphosphorylated peptide were obtained which lacked any line broadening in the presence of thrombin (data not shown).

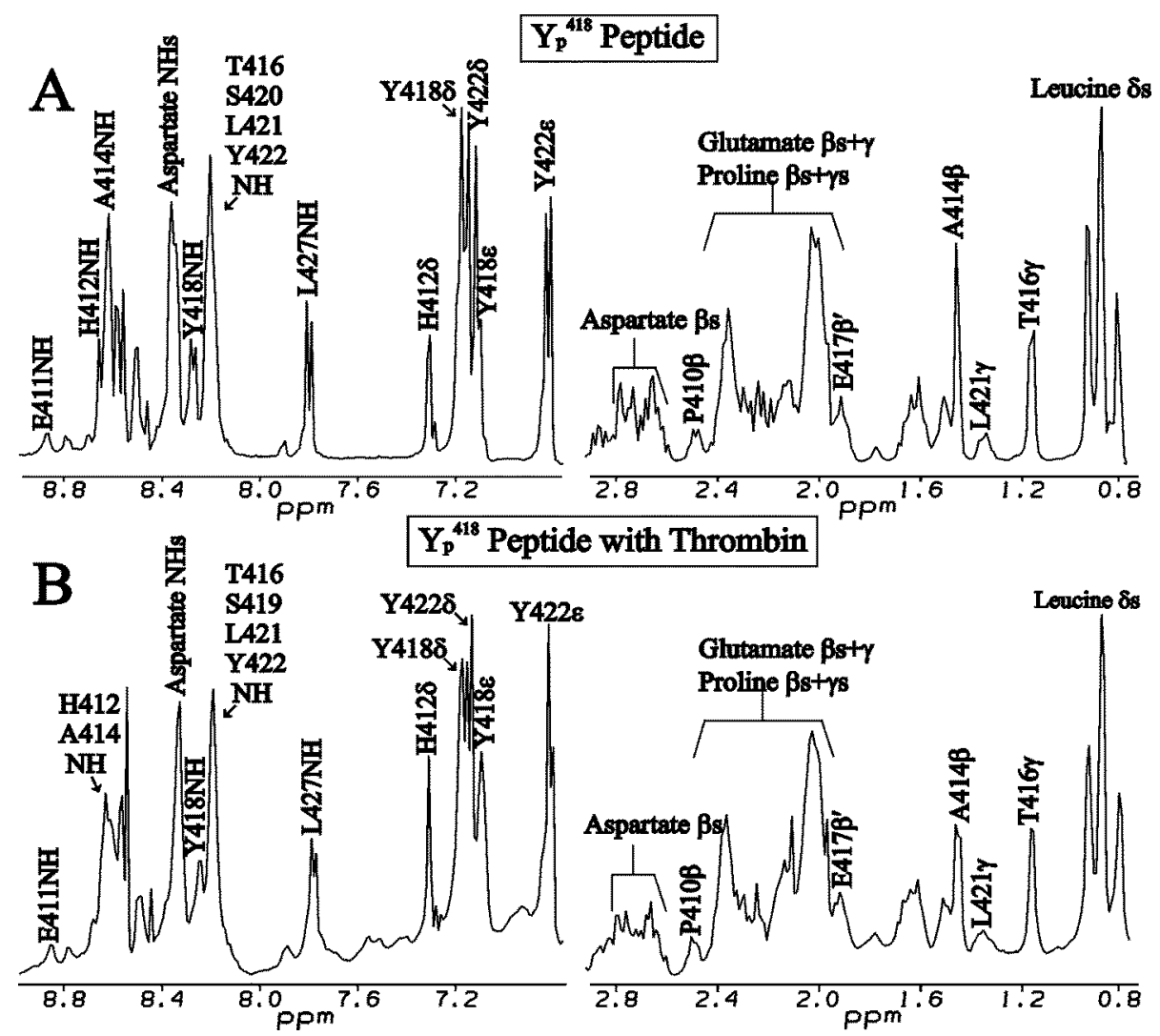

Figure 22: Line broadening for the $\gamma^{\prime}\left(\mathrm{Y}_{\mathrm{P}}^{418}\right)$ peptide in the presence of thrombin. A. 1D NMR spectrum for $1.5 \mathrm{mM}$ peptide in solution. B. 1D NMR spectrum for $1.5 \mathrm{mM}$ peptide in the presence of $0.151 \mathrm{mM}$ thrombin. The experimental conditions are the same as in Figure 27. A significant reduction in line broadening occurs in the absence of phosphorylation at $\mathrm{Y}^{422}$. Residues displaying contact with the enzyme include $\mathrm{H}^{412}, \mathrm{~A}^{414}, \mathrm{E}^{417}, \mathrm{Y}^{418}, \mathrm{E}^{424}, \mathrm{~L}^{427}$, and the aspartate $\beta$ s.

Two-Dimensional Transferred Nuclear Overhauser Effect NMR. For the $\gamma^{\prime}$ peptides representing $\mathrm{Y}_{\mathrm{P}}^{418} \mathrm{Y}_{\mathrm{P}}^{422}, \mathrm{Y}_{\mathrm{P}}^{418}$, and $\mathrm{Y}_{\mathrm{P}}^{422}$, between 135-155 inter-residue NOEs were observed in the presence of IIa displaying similar structural features. Appendix A lists the chemical shifts associated with each residue in all four $\gamma^{\prime}$ peptides. In addition, interresidue NOEs for the $\mathrm{Y}_{\mathrm{P}}^{418} \mathrm{Y}_{\mathrm{P}}^{422}, \mathrm{Y}_{\mathrm{P}}^{418}$, and $\mathrm{Y}_{\mathrm{P}}{ }^{422}$ peptides are compiled in Appendix B. By removing both phosphates from the peptide, 26 inter-residue, nearest neighbor NOEs were identified in the presence of IIa, which is quite similar to the NOESY of the free 
phosphorylated peptides (data not shown). This significant decrease in NOEs suggests that phosphorylation of at least one $\mathrm{Y}$ residue is required for interacting effectively with IIa. Such an effect is in agreement with the 1D line broadening studies.

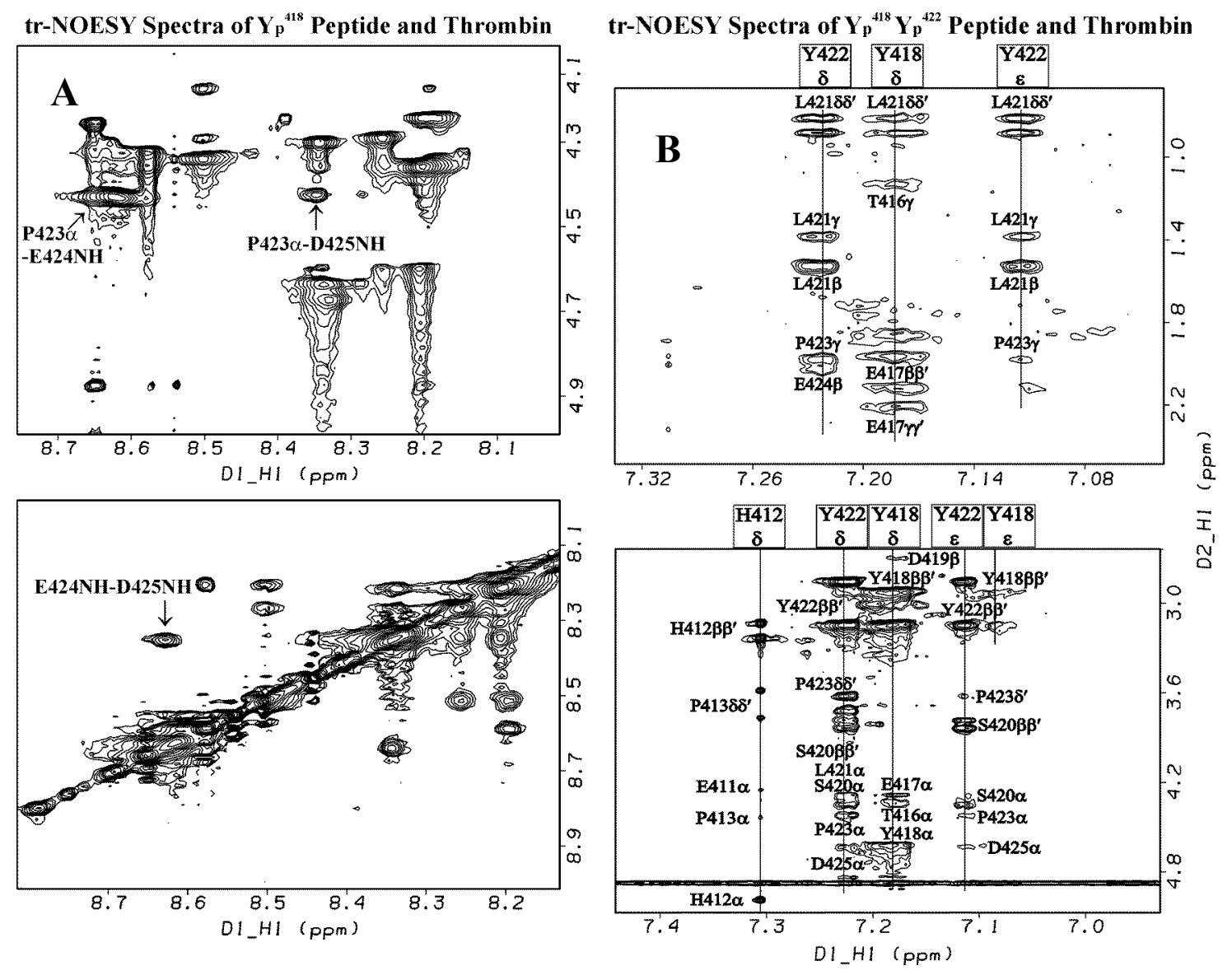

Figure 23: Representative spectra of key $\gamma^{\prime}$ peptide NOEs in the presence of thrombin. A. $\beta$ turn between residues $\mathrm{Y}^{422}-\mathrm{D}^{425}$ from $2 \mathrm{D}$ trNOESY spectrum of the $\mathrm{Y}_{\mathrm{P}}^{418}$ peptide $(1.5 \mathrm{mM})$ in the presence of IIa $(0.151 \mathrm{mM})$. All three phosphorylated peptides exhibit this conformational feature, while the turn is absent when analyzing spectra involving the unphosphorylated peptide. $B$. The intense clustering of NOEs centered at the aromatic protons of both tyrosine residues. The spectrum is from the 2D trNOESY of $\mathrm{Y}_{\mathrm{P}}^{418} \mathrm{Y}_{\mathrm{P}}^{422}$ peptide $(1.5 \mathrm{mM})$ in the presence of IIa $(0.162 \mathrm{mM})$. The aromatic protons of $\mathrm{Y}^{418}$ are in close proximity to aliphatic protons from $\mathrm{T}^{416}$, $\mathrm{E}^{417}, \mathrm{D}^{419}$, and $\mathrm{L}^{421}$, while the $\mathrm{Y}^{422}$ ring protons display NOEs with residues $\mathrm{S}^{420}, \mathrm{~L}^{421}, \mathrm{P}^{423}, \mathrm{E}^{424}$, and $\mathrm{D}^{425}$. When the unphosphorylated peptide is observed, the NOEs are no longer present.

Important structural information related to the conformation of all three phosphorylated peptides bound to IIa are obtained through analysis of the trNOESY spectra. Key through space interactions occurs between the $\mathrm{H}^{412} \alpha-\mathrm{P}^{413} \delta$ s protons, as 
well as the $\mathrm{Y}^{422} \beta-\mathrm{P}^{423} \delta$ s protons (see Appendix B). These NOEs are indicative of an Xxx-Pro bond in the trans conformation (249). Figure 23A from the trNOESY spectrum of $\mathrm{Y}_{\mathrm{P}}^{418}$ displays a $\beta$-turn formed by residues $\mathrm{Y}^{422}-\mathrm{E}^{425}$. The principal NOEs for this turn are between the $\mathrm{P}^{423} \alpha-\mathrm{E}^{424} \mathrm{NH}$, the $\mathrm{P}^{423} \alpha-\mathrm{D}^{425} \mathrm{NH}$, and the NHs of $\mathrm{E}^{424}$ and $\mathrm{D}^{425}(249)$. The NOEs for the $\beta$-turn and the proline trans bonds were also present in the $2 \mathrm{D}$ spectrum of the $\mathrm{Y}_{\mathrm{P}}^{418} \mathrm{Y}_{\mathrm{P}}{ }^{422}$ peptide binding to $\gamma$-IIa (a form of IIa with an impaired ABEI, see Chapter I) (data not shown). Examining the trNOESY spectrum for the unphosphorylated peptide with native IIa reveals the existence of trans $\mathrm{H}^{412}-\mathrm{P}^{413}$, but the absence of the required NOEs for the $\beta$-turn and the C-terminal trans bond.

A region displaying numerous proton interactions in the trNOESY spectra of the three phosphorylated peptides is centered on the two aromatic $\mathrm{Y}$ residues (Figure 23B and Appendix B). A hydrophobic cluster, or an intense grouping of aliphatic protons, is present encompassing $\mathrm{T}^{416}-\mathrm{D}^{425}$. By including through space interactions between $\mathrm{E}^{424} \alpha$ and the aliphatic chain of $\mathrm{L}^{427}$, this cluster extends to the $\mathrm{C}$-terminus of the peptide.

${ }^{31} \mathrm{P}$ NMR Studies. Phosphorous NMR spectra were collected for the $\mathrm{Y}_{\mathrm{P}}^{418} \mathrm{Y}_{\mathrm{P}}{ }^{422}$, $\mathrm{Y}_{\mathrm{P}}^{418}$, and $\mathrm{Y}_{\mathrm{P}}^{422}$ peptides with and without IIa in solution. These experiments were performed to monitor the effects of peptide-IIa interaction on line broadening. The $\mathrm{Y}_{\mathrm{P}}{ }^{418} \mathrm{Y}_{\mathrm{P}}{ }^{422}$ peptide displayed two resonances at $-3.06 \mathrm{ppm}$ and $-3.14 \mathrm{ppm}$ (Figure 24). Addition of IIa resulted in a merging of both phosphorous signals into one peak with a significant amount of line broadening. The singly phosphorylated peptides were also analyzed to assess the contribution each individual $Y_{P}$ makes to the ${ }^{31} \mathrm{P}$ NMR spectrum. The $Y_{P}^{418}$ and $Y_{P}{ }^{422}$ peptide signals shift upfield and become slightly broader with the introduction of IIa (data not shown). 

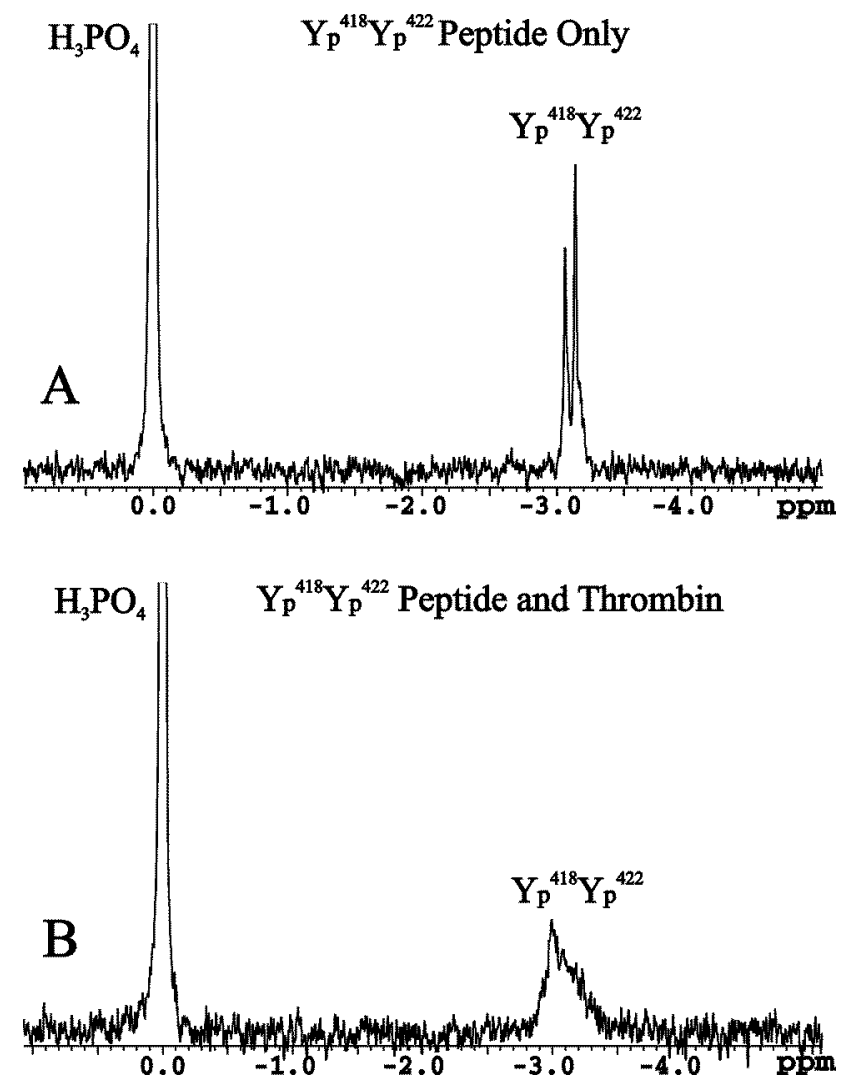

Figure 24: ${ }^{31} \mathrm{P}$ 1D NMR spectra for the $\gamma^{\prime} \mathrm{Y}_{\mathrm{P}}^{418} \mathrm{Y}_{\mathrm{P}}{ }^{422}$ peptide in the presence of thrombin. A. Spectrum for $1.5 \mathrm{mM}$ peptide in solution. B. Spectrum for $1.5 \mathrm{mM}$ peptide in the presence of $0.162 \mathrm{mM}$ IIa. Both phosphotyrosines are contacting the enzyme surface resulting in a slight downfield shift of the broadened peak.

HDX Experiments: Examining the Influence of the $\gamma^{\prime}$ Peptide on the Solvent Accessibility of Thrombin. Peptic digests of thrombin, with and without the reducing agent TCEP, yielded fourteen quantifiable isotopic clusters encompassing $44 \%$ of thrombin's sequence (Figure 25). The presence of four disulfide bonds $\left(C^{1}-C^{122}, C^{42}-C^{58}\right.$, $\mathrm{C}^{168}-\mathrm{C}^{182}$, and $\mathrm{C}^{191}-\mathrm{C}^{220}$ ) limits the amount of sequence coverage attainable without introducing a reducing agent. Peptides spanning ABE-1 (65-84), ABE-2 (85-99), the autolysis loop (135-149D), and several residues involved in substrate specificity $\left(\mathrm{L}^{99}\right.$ and $\mathrm{I}^{174}$ ) are represented in the peptic digests. TCEP allowed additional coverage to be obtained in the A-chain (-3 to 7), as well as a region encompassing $\mathrm{W}^{215}$ and the $\mathrm{Na}^{+}$ binding site (212-227). Appendix C lists the $\mathrm{m} / \mathrm{z}$ values for the fragments obtained from a peptic digest of thrombin. 


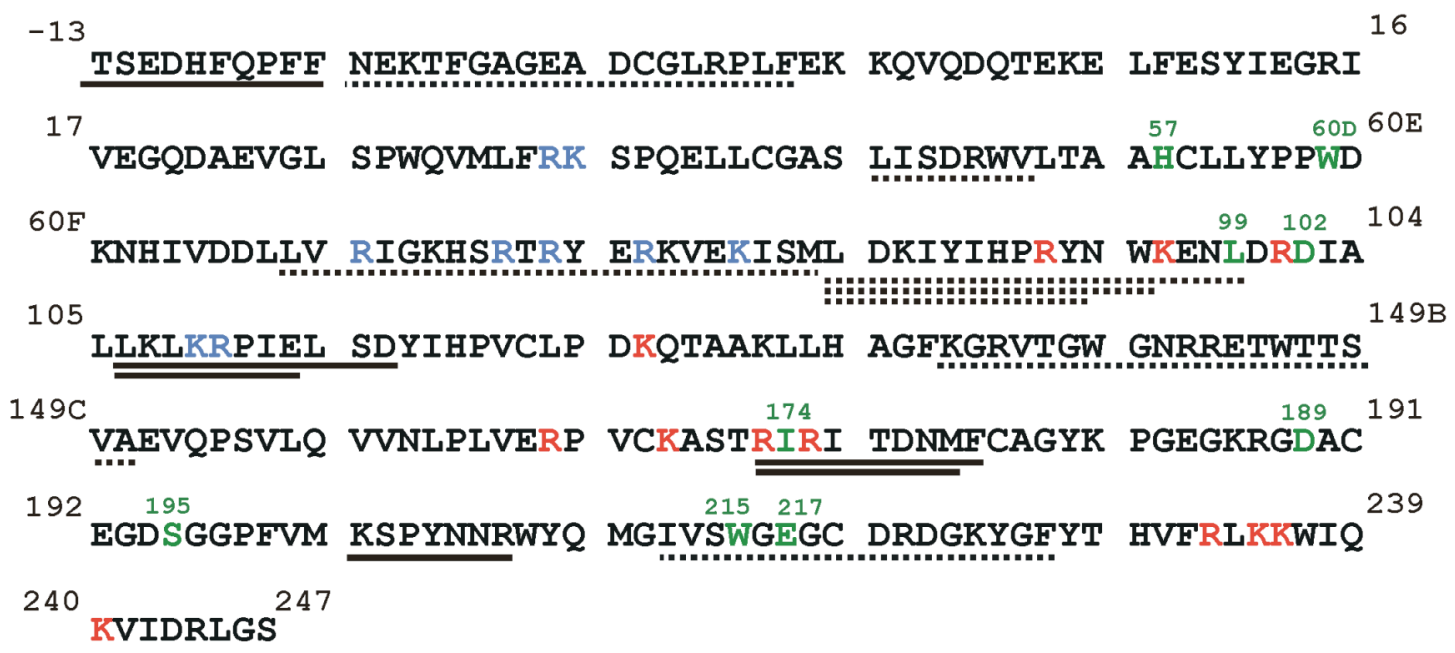

Figure 25: Sequence coverage from the peptic digest of bovine thrombin. The solid black lines indicate quantifiable peptide clusters obtained without a reducing agent, while dashed lines refer to peptides acquired in the presence of TCEP. This coverage represents $44 \%$ of the total sequence and includes residues from both anion binding exosites, the $\mathrm{W}^{148}$ loop, the $\mathrm{Na}^{+}$binding site, and several residues involved in substrate specificity $\left(\mathrm{L}^{99}, \mathrm{I}^{174}\right.$, and $\left.\mathrm{W}^{215}\right)$. Residues in the catalytic triad are green. ABE-I amino acids are blue and ABE-II residues are red.

IIa with or without the addition of $1 \mathrm{mM} \gamma^{\prime}$ peptide was exposed to $100 \% \mathrm{D}_{2} \mathrm{O}$ for either 1 or 10 minutes. The concentration of $1 \mathrm{mM} \gamma^{\prime}$ peptide provided at least $99.93 \%$ occupancy of $40-50 \mu \mathrm{M}$ IIa $\left(\mathrm{K}_{\mathrm{D}}=0.68 \mu \mathrm{M}\right)(69)$. Table 4 and Figure 26 present the raw data representing total deuterium incorporation for IIa. The fourteen quantifiable peptides represent ten distinct regions within IIa (Figure 25). When analyzing IIa alone, five of these regions were continuing to exchange for deuterium after 10 minutes: 85-99, 106-116, 173-181, 202-207, and 212-227. These residues span ABE-II, the $\mathrm{Na}^{+}$binding site, and the substrate specificity residues $\mathrm{L}^{99}, \mathrm{I}^{174}$, and $\mathrm{W}^{215}$. The other five segments, representing ABE-I, the A-chain, and the autolysis loop, became saturated with deuterium at 1 minute: -13 to $-4,-3$ to $7,46-52,65-84$, and 135-149D. The continued uptake of deuterium over the entire observed time range is specific to amide protons more protected from the solvent, or partially solvent exposed. Conversely, almost complete initial saturation of the peptide indicates a more solvent exposed region (229). 


\section{Deuterium Incorporation at 1 Minute}

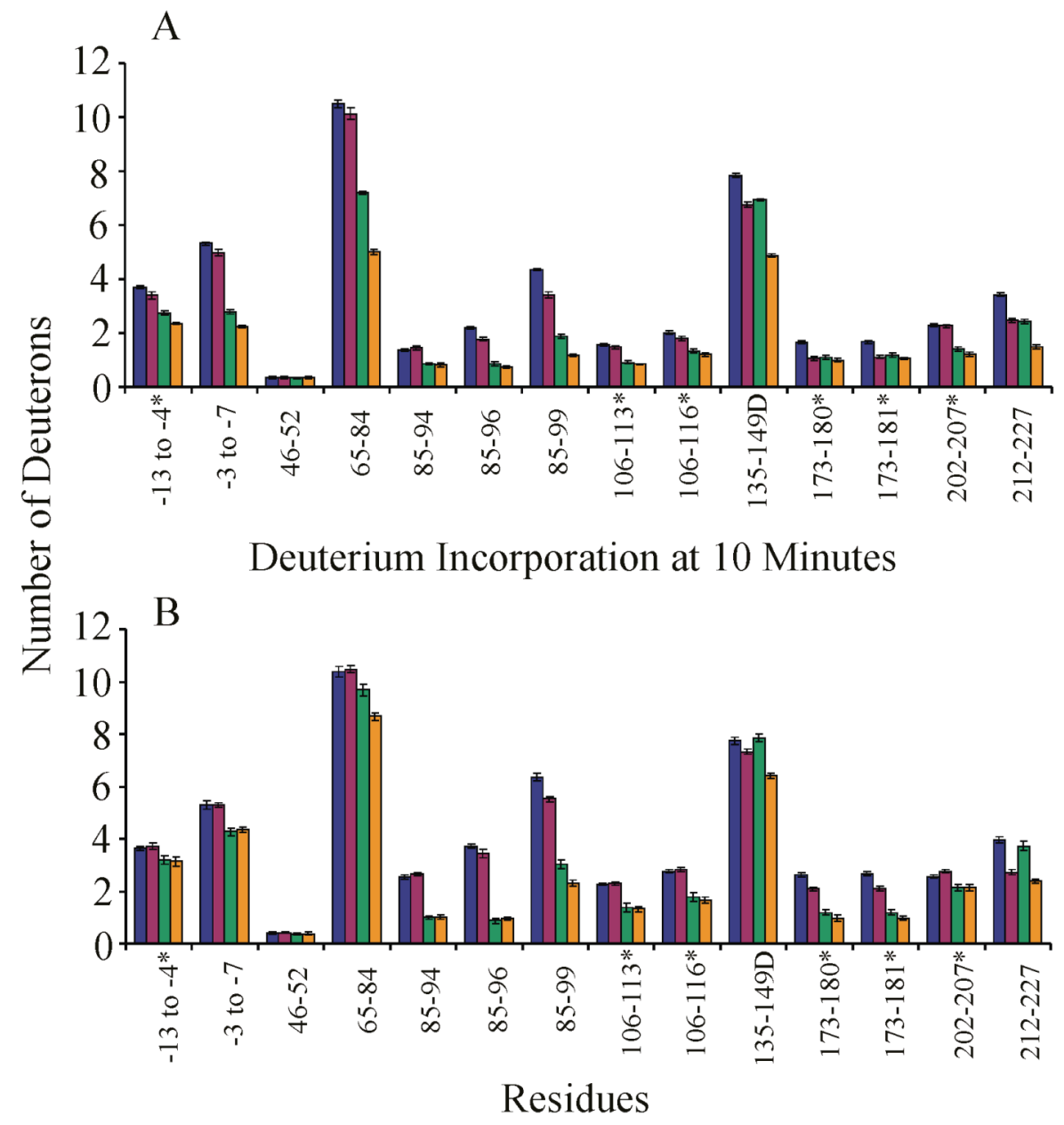

Figure 26: Graphs of deuterium incorporation for IIa, PPACK-IIa, $\gamma^{\prime}$-IIa, and $\gamma^{\prime}$ PPACK-IIa. A. one minute and B. ten minutes of HDX for IIa in the absence or presence of ligands. The bars in the graph correspond to: IIa (purple), PPACK-IIa (magenta), $\gamma^{\prime}$ peptide-IIa (green), and $\gamma^{\prime}$ peptide PPACK-IIa (orange). The asterisk symbol (*) located after 6 fragments on the $\mathrm{x}$-axis refers to data obtained in the absence of TCEP for fragments. Errors correspond to standard deviation of the mean for three independent experiments.

Differences in Deuteration for Thrombin versus $\gamma^{\prime}$ Peptide-Thrombin. The $\gamma^{\prime}$ peptide imparts a significant amount of solvent protection (Table 5; Figure 26) in five IIa peptides representing two regions within ABE-II. Peptides 85-94, 85-96, and 85-99 are situated in the center of ABE-II. Peptides 173-180 and 173-181 involve peripheral ABEII residues and the substrate specificity residue $\mathrm{I}^{174}$. After ten minutes, differences in HDX for these ABE-II regions significantly increase when compared to one minute 
(Table 5). For example, $\gamma^{\prime}$ peptide binding within 85-94 leads to HDX protection of 0.5

deuterons at 1 minute $(-5.6 \%)$, which increases to 1.6 deuterons at 10 minutes $(-17.7 \%)$

(Figure 27).

\begin{tabular}{|c|c|c|c|c|c|}
\hline \multirow{2}{*}{ Residues } & \multirow{2}{*}{$\begin{array}{l}\text { Theo. } \\
\mathrm{D}_{\max }{ }^{a}\end{array}$} & \multicolumn{4}{|c|}{1 Minute } \\
\hline & & IIa & P-IIa & $\gamma^{\prime}$ IIa & $\gamma^{\prime}$ P-IIa \\
\hline-13 to $-4^{b}$ & 8.5 & $3.7 \pm 0.1$ & $3.4 \pm 0.1$ & $2.7 \pm 0.1$ & $2.3 \pm 0.0$ \\
\hline-3 to 7 & 16.9 & $5.3 \pm 0.1$ & $5.0 \pm 0.1$ & $2.8 \pm 0.1$ & $2.3 \pm 0.0$ \\
\hline $46-52$ & 6.6 & $0.4 \pm 0.0$ & $0.4 \pm 0.0$ & $0.3 \pm 0.0$ & $0.4 \pm 0.0$ \\
\hline $65-84$ & 21.8 & $10.5 \pm 0.1$ & $10.1 \pm 0.2$ & $7.2 \pm 0.1$ & $5.0 \pm 0.1$ \\
\hline $85-94$ & 8.8 & $1.4 \pm 0.0$ & $1.5 \pm 0.1$ & $0.9 \pm 0.0$ & $0.8 \pm 0.1$ \\
\hline $85-96$ & 10.9 & $2.2 \pm 0.1$ & $1.8 \pm 0.1$ & $0.9 \pm 0.1$ & $0.7 \pm 0.0$ \\
\hline $85-99$ & 15.2 & $4.4 \pm 0.0$ & $3.4 \pm 0.1$ & $1.9 \pm 0.1$ & $1.2 \pm 0.0$ \\
\hline $106-113^{b}$ & 6.7 & $1.6 \pm 0.0$ & $1.5 \pm 0.1$ & $0.9 \pm 0.1$ & $0.9 \pm 0.0$ \\
\hline $106-116^{b}$ & 9.8 & $2.0 \pm 0.1$ & $1.8 \pm 0.1$ & $1.3 \pm 0.1$ & $1.2 \pm 0.1$ \\
\hline 135-149D & 19.4 & $7.8 \pm 0.1$ & $6.8 \pm 0.1$ & $6.9 \pm 0.0$ & $4.9 \pm 0.1$ \\
\hline $173-180^{b}$ & 7.8 & $1.7 \pm 0.1$ & $1.1 \pm 0.1$ & $1.1 \pm 0.1$ & $1.0 \pm 0.1$ \\
\hline $173-181^{b}$ & 8.8 & $1.7 \pm 0.1$ & $1.1 \pm 0.1$ & $1.2 \pm 0.1$ & $1.1 \pm 0.1$ \\
\hline $202-207^{b}$ & 6.8 & $2.3 \pm 0.0$ & $2.3 \pm 0.0$ & $1.4 \pm 0.1$ & $1.2 \pm 0.1$ \\
\hline $212-227$ & 15.9 & $3.4 \pm 0.1$ & $2.5 \pm 0.1$ & $2.4 \pm 0.1$ & $1.5 \pm 0.1$ \\
\hline \multirow{2}{*}{ Residues } & \multirow{2}{*}{$\begin{array}{l}\text { Theo. } \\
\mathrm{D}_{\max }\end{array}$} & \multicolumn{4}{|c|}{10 Minutes } \\
\hline & & IIa & P-IIa & $\gamma^{\prime}$ IIa & $\gamma^{\prime}$ P-IIa \\
\hline-13 to $-4^{b}$ & 8.5 & $3.6 \pm 0.1$ & $3.7 \pm 0.1$ & $3.2 \pm 0.2$ & $3.1 \pm 0.2$ \\
\hline-3 to 7 & 16.9 & $5.3 \pm 0.1$ & $5.3 \pm 0.1$ & $4.3 \pm 0.1$ & $4.4 \pm 0.1$ \\
\hline $46-52$ & 6.6 & $0.4 \pm 0.0$ & $0.4 \pm 0.0$ & $0.4 \pm 0.0$ & $0.4 \pm 0.1$ \\
\hline $65-84$ & 21.8 & $10.4 \pm 0.2$ & $10.5 \pm 0.1$ & $9.7 \pm 0.2$ & $8.7 \pm 0.2$ \\
\hline $85-94$ & 8.8 & $2.6 \pm 0.1$ & $2.7 \pm 0.1$ & $1.0 \pm 0.1$ & $1.0 \pm 0.1$ \\
\hline $85-96$ & 10.9 & $3.7 \pm 0.1$ & $3.4 \pm 0.2$ & $0.9 \pm 0.1$ & $1.0 \pm 0.1$ \\
\hline $85-99$ & 15.2 & $6.4 \pm 0.1$ & $5.5 \pm 0.1$ & $3.0 \pm 0.2$ & $2.3 \pm 0.1$ \\
\hline $106-113^{b}$ & 6.7 & $2.3 \pm 0.0$ & $2.3 \pm 0.1$ & $1.4 \pm 0.2$ & $1.3 \pm 0.1$ \\
\hline $106-116^{b}$ & 9.8 & $2.8 \pm 0.1$ & $2.8 \pm 0.1$ & $1.8 \pm 0.2$ & $1.7 \pm 0.1$ \\
\hline 135-149D & 19.4 & $7.7 \pm 0.1$ & $7.3 \pm 0.1$ & $7.8 \pm 0.1$ & $6.4 \pm 0.1$ \\
\hline $173-180^{b}$ & 7.8 & $2.6 \pm 0.1$ & $2.1 \pm 0.1$ & $1.2 \pm 0.1$ & $1.0 \pm 0.1$ \\
\hline $173-181^{b}$ & 8.8 & $2.7 \pm 0.1$ & $2.1 \pm 0.1$ & $1.2 \pm 0.1$ & $1.0 \pm 0.1$ \\
\hline $202-207^{b}$ & 6.8 & $2.6 \pm 0.1$ & $2.8 \pm 0.1$ & $2.2 \pm 0.1$ & $2.1 \pm 0.1$ \\
\hline $212-227$ & 15.9 & $4.0 \pm 0.1$ & $2.7 \pm 0.1$ & $3.7 \pm 0.2$ & $2.4 \pm 0.1$ \\
\hline
\end{tabular}

${ }^{a}$ The maximum number of exchangeable protons within the indicated peptide, assuming $100 \%$ deuteration. This value accounts for all exchangeable backbone amide protons and a slight fraction of $\mathrm{N}$-terminal, $\mathrm{C}$-terminal, and side chain exchangeable protons that are dependent on the final percentage of $\mathrm{D}_{2} \mathrm{O}$ in solution under quench conditions (approximately 4.5\%). A fully deuterated peptide would theoretically have acquired this amount of deuterons. ${ }^{b}$ The deuterium uptake for these peptides was quantified from the non-reducing digests. All other peptides are from the digests with TCEP. When isotopic clusters appear in both the TCEP and nonTCEP digests, the differences in deuteration are negligible when comparing similar peptides. Certain peptides appear exclusively under non-reducing or reducing conditions, hence the necessity of performing both types of peptic digests. 
Residues 85-94

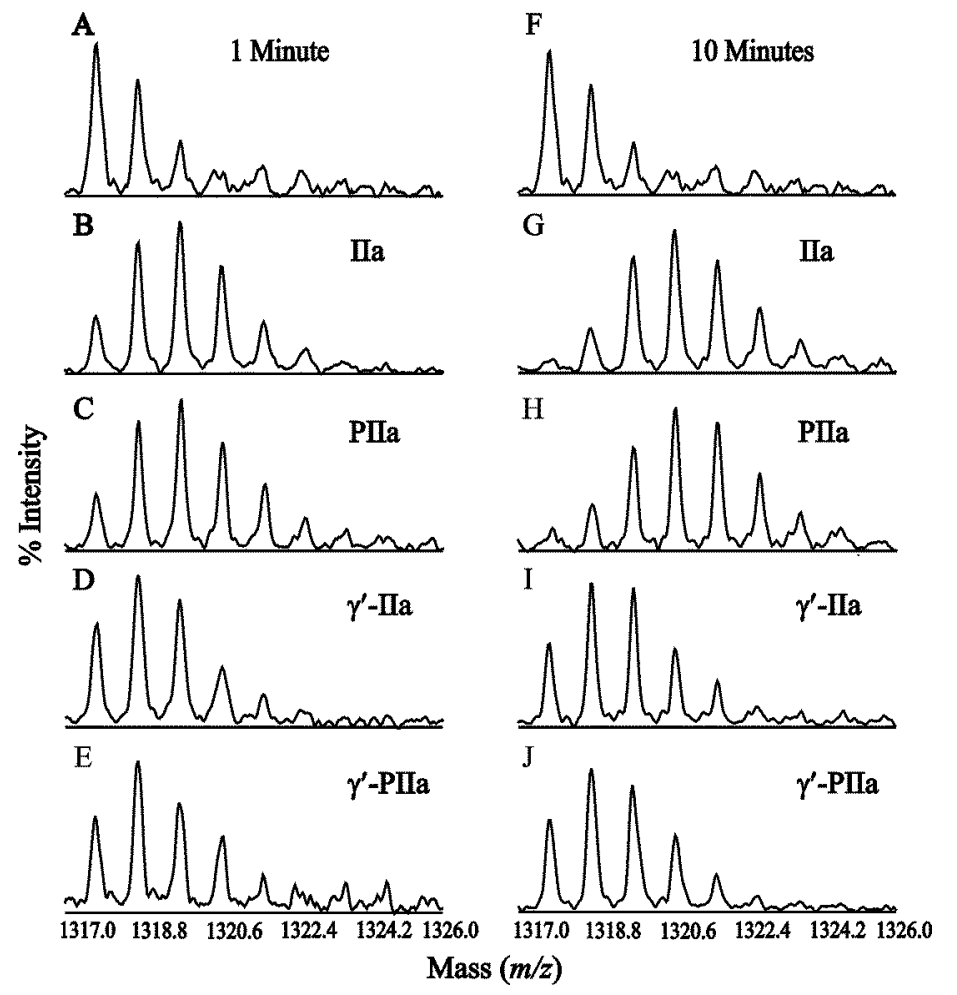

Figure 27: Mass spectra of IIa residues 85-94 after one and ten minutes of HDX. All D-on experiments are in $150 \mathrm{mM} \mathrm{NaCl}$, $25 \mathrm{mM} \mathrm{NaH}_{2} \mathrm{PO}_{3}, \mathrm{pH} 6.5$ (HDX buffer). $\mathrm{A}$ and $\mathrm{F}$ : the undeuterated peak cluster, $B$ and $G$ : thrombin spectrum in the absence of ligands, $\mathrm{C}$ and $\mathrm{H}$ : PPACK inhibited thrombin, D and I: $\gamma^{\prime}$ peptide bound to thrombin, and $\mathrm{E}$ and J: $\gamma^{\prime}$ peptide bound to PPACK inhibited thrombin. This peak cluster contains the ABE-II residue $\mathrm{R}^{93}$ and experiences a significant degree of protection from deuterium in the presence of the $\gamma^{\prime}$ peptide. This protection from solvent is maintained over the course of ten minutes.

In addition to the noted protection within the vicinity of ABE-II, the $\gamma^{\prime}$ peptide also shielded regions distant from ABE-II. Unlike the ABE-II fragments, these thrombin segments lost some of their degree of protection as the incubation with $\mathrm{D}_{2} \mathrm{O}$ was increased from 1 to 10 minutes. Such thrombin segments are likely not becoming completely solvent excluded in the presence of the $\gamma^{\prime}$ peptide. The regions affected include ABE-I (65-84 and 106-116), the A-chain (-13 to -4 and -3 to 7 ) and a nearby Bchain loop (202-207), the $\mathrm{W}^{215}$ region (212-227), and the autolysis loop (135-149D). See Table 5 and also Figure 26. Figures 28B and 28D illustrate that within one minute the isotopic cluster representing residues within ABE-I (65-84) underwent a $-15.1 \%$ difference in deuteration relative to free thrombin whereas the cluster for residues within the $\mathrm{W}^{215}$ region (212-227) underwent a $-6.3 \%$ difference. Fragment $46-52$ of thrombin remained insensitive to the $\gamma^{\prime}$ peptide binding to ABE-II. 
Figure 28: Mass spectra of IIa residues 65-84 and 212-227 after one minute of HDX. All D-on experiments are in $150 \mathrm{mM} \mathrm{NaCl}, 25$ $\mathrm{mM} \mathrm{NaH}{ }_{2} \mathrm{PO}_{3}$, pH 6.5 (HDX buffer). $\mathrm{A}$ : the undeuterated peak cluster, $\mathrm{B}$ : thrombin spectrum in the absence of ligands, C: PPACK inhibited thrombin, D: $\gamma^{\prime}$ peptide bound to thrombin, and E: $\gamma^{\prime}$ peptide bound to PPACK inhibited thrombin.

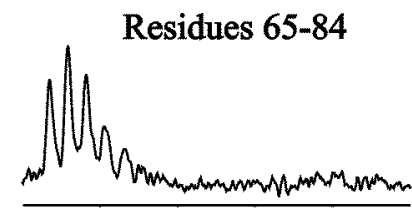

A
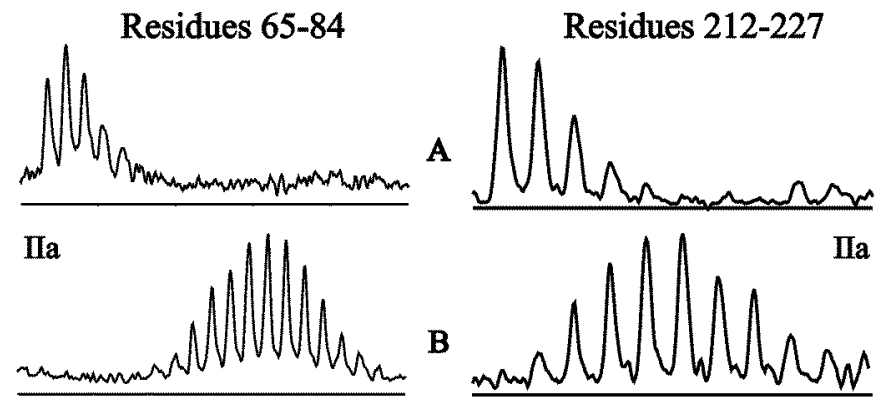

PIIa

IIa

B

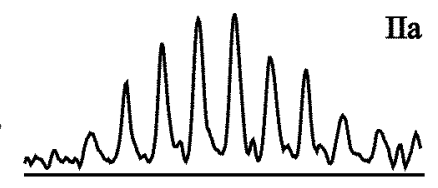

PIIa
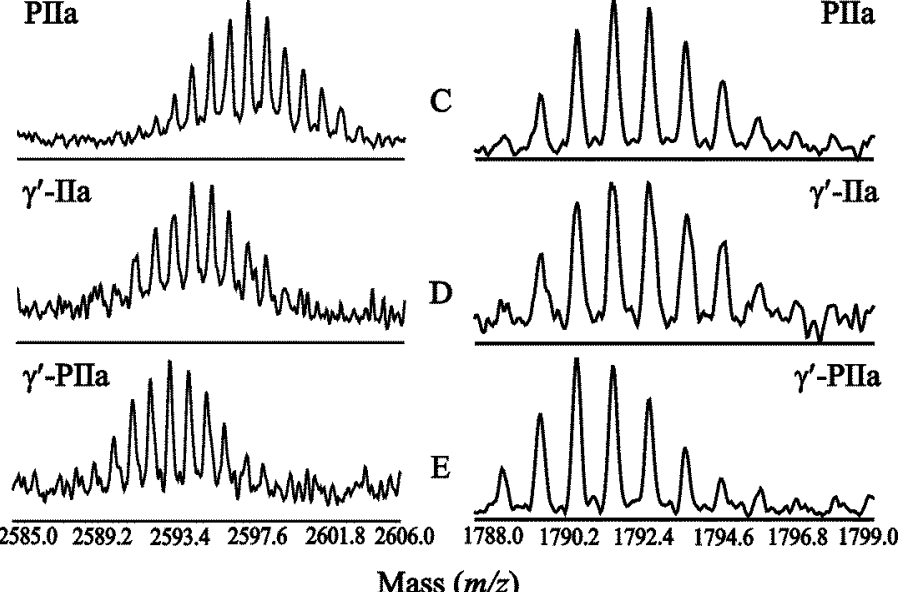

Mass $(m / z)$

Differences in Deuteration Between PPACK-Thrombin and the $\gamma^{\prime}$ Peptide-PPACK-

Thrombin Complex. $\gamma^{\prime}$ peptide binding to PPACK-IIa resulted in significant protection from HDX for almost every IIa region (Tables 4 and 5; Figure 26). After 10 minutes of HDX, the peptide $85-99(2102.12 \mathrm{~m} / \mathrm{z})$ experiences a decrease in deuteration of $-21.8 \%$ in the presence of the $\gamma^{\prime}$ peptide, a decrease of $-5.5 \%$ with PPACK inhibition, and both interacting together with IIa result in a decrease of $-26.6 \%$ (Table 5). These additive shifts lead to lower $\mathrm{m} / \mathrm{z}$ values for the peptide as can be seen by comparing the isotopic clusters of PPACK-IIa, $\gamma^{\prime}$ peptide-IIa, and $\gamma^{\prime}$ peptide-PPACK IIa (Figures 27 and 28).

Located adjacent to this ABE-II region is the fragment 173-181. This segment encompasses several residues involved in substrate specificity $\left(\mathrm{R}^{173}\right.$ and $\left.\mathrm{I}^{174}\right)$ and some peripheral ABE-II residues $\left(\mathrm{R}^{173}\right.$ and $\left.\mathrm{R}^{175}\right)$. When examining the results for one minute of HDX, the binary and ternary complexes with IIa offer similar degrees of protection 
(between $-5.9 \%$ and $-7.1 \%$ ) (Table 5). After ten minutes of HDX, differences are more apparent. The $\gamma^{\prime}$ peptide (-16.9\%) appears capable of imparting an increasing degree of protection over 10 minutes whereas PPACK's affect remains at similar levels (-6.6\%).

Another region of thrombin for which there are some time dependent trends in deuteration involves residues 212-227 (Table 5). Shifts to this peptide's isotopic cluster can be followed in Figure 28. After one minute of HDX, both PPACK and the $\gamma^{\prime}$ peptide impart a similar degree of protection from solvent (-6.0\% and $-6.3 \%)$. Protection of the ternary $\gamma^{\prime}$-PPACK-IIa complex appears additive at (-12.1\%). Similarities in protection for the binary complexes, however, are lost within 10 minutes of HDX. PPACK-IIa now exhibits protection of $(-7.9 \%)$, whereas $\gamma^{\prime}$ peptide binding no longer significantly affects this region $(-1.5 \%)$. Interestingly, the degree of protection of the ternary complex is still roughly additive (-10.0\%). Contrary to results for segment $173-181$, it is the PPACK, not the $\gamma^{\prime}$ peptide, which dominates in hindering exchange for segment 212-227.

Two solvent exposed regions encompassing ABE-I (65-84) and the autolysis loop (135-149D) present evidence of a synergistic effect involving the interactions of both ligands (Table 5). After one minute of HDX, the presence of PPACK affords very little HDX protection for $65-84$ (-1.6\%) (Figure 28C), whereas the $\gamma^{\prime}$ peptide provides significant protection (-15.1\%) (Figure 28B). Interestingly, both ligands together offer even more protection from solvent than an additive effect would suggest (-25.1\%) (Figure 28D). The same is true for the segment spanning 135-149D. The presence of both these ligands appears to stabilize these surface regions of IIa, protecting the backbone amides from exchange more effectively than either ligand acting alone. 
The peptides spanning residues -13 to $7,106-116$, and 202-207 experienced roughly similar protection when the $\gamma^{\prime}$ peptide bound to ABE-II in the presence or absence of PPACK (Tables 4 and 5). PPACK's sphere of influences must not extend to these fragments. The A-chain peptides -13 to -4 and -3 to 7 incorporate an almost identical amount of deuterium at one minute and after ten minutes display similar \% differences (ranging from $-5.1 \%$ to $-6.1 \%$ ). Located in close proximity to the A-chain, the fragment 202-207 was also protected from solvent within ten minutes of deuteration by a comparable amount to the A-chain (-6.1\%). Finally, peptides 106-113 and 106-116, containing several peripheral ABE-I residues, incorporated virtually identical amounts of deuterium regardless of the presence of PPACK.

Table 5: Changes in \% Deuteration for IIa, PPACK-IIa, $\gamma^{\prime}$-IIa, and $\gamma^{\prime}$ PPACK-IIa Complexes at One and Ten Minutes Relative to Free IIa ${ }^{a}$

\begin{tabular}{cc|cc|cc|cc}
\hline \multirow{2}{*}{ Residues } & Theo. & \multicolumn{2}{|c|}{ P-IIa } & \multicolumn{2}{c|}{$\gamma^{\prime}$ IIa } & \multicolumn{2}{c}{$\gamma^{\prime}$ P-IIa } \\
\cline { 3 - 7 } & $D_{\max }{ }^{2}$ & 1 min & $10 \min$ & 1 min & 10 min & 1 min & 10 min \\
\hline-13 to $-4^{c}$ & 8.5 & -3.6 & 0.8 & $-\mathbf{1 1 . 3}$ & $\mathbf{- 5 . 1}$ & $\mathbf{- 1 5 . 9}$ & $\mathbf{- 5 . 8}$ \\
-3 to 7 & 16.9 & -2.0 & 0.0 & $-\mathbf{1 5 . 0}$ & $\mathbf{- 6 . 1}$ & $\mathbf{- 1 8 . 2}$ & $\mathbf{- 5 . 6}$ \\
$46-52$ & 6.6 & 0.0 & 0.0 & -0.2 & -0.7 & 0.1 & -0.6 \\
$65-84$ & 21.8 & -1.6 & 0.4 & $\mathbf{- 1 5 . 1}$ & -3.3 & $\mathbf{- 2 5 . 1}$ & $-\mathbf{7 . 9}$ \\
$85-94$ & 8.8 & 1.1 & 1.2 & $\mathbf{- 5 . 6}$ & $\mathbf{- 1 7 . 7}$ & $\mathbf{- 6 . 2}$ & $\mathbf{- 1 7 . 6}$ \\
$85-96$ & 10.9 & -3.9 & -2.5 & $\mathbf{- 1 2 . 3}$ & $\mathbf{- 2 5 . 9}$ & $\mathbf{- 1 3 . 4}$ & $\mathbf{- 2 5 . 2}$ \\
$85-99$ & 15.2 & $\mathbf{- 6 . 3}$ & $\mathbf{- 5 . 5}$ & $\mathbf{- 1 6 . 4}$ & $\mathbf{- 2 1 . 8}$ & $\mathbf{- 2 0 . 9}$ & $\mathbf{- 2 6 . 6}$ \\
$106-113^{c}$ & 6.7 & -1.6 & -0.5 & $\mathbf{- 1 0 . 0}$ & $\mathbf{- 1 3 . 1}$ & $\mathbf{- 1 0 . 8}$ & $\mathbf{- 1 3 . 9}$ \\
$106-116^{c}$ & 9.8 & -2.1 & -0.6 & $\mathbf{- 6 . 9}$ & $\mathbf{- 1 0 . 0}$ & $\mathbf{- 8 . 3}$ & $\mathbf{- 1 1 . 2}$ \\
$135-149 \mathrm{D}$ & 19.4 & $\mathbf{- 5 . 5}$ & -2.2 & $\mathbf{- 4 . 6}$ & -0.5 & $\mathbf{- 1 5 . 2}$ & $\mathbf{- 6 . 9}$ \\
$173-180^{c}$ & 7.8 & $\mathbf{- 7 . 7}$ & $\mathbf{- 7 . 0}$ & $\mathbf{- 7 . 3}$ & $\mathbf{- 1 8 . 6}$ & $\mathbf{- 8 . 7}$ & $\mathbf{- 2 1 . 3}$ \\
$173-181^{c}$ & 8.8 & $\mathbf{- 6 . 6}$ & $\mathbf{- 6 . 6}$ & $\mathbf{- 5 . 9}$ & $\mathbf{- 1 6 . 9}$ & $\mathbf{- 7 . 1}$ & $\mathbf{- 1 9 . 3}$ \\
$202-207^{c}$ & 6.8 & 0.5 & 2.9 & $\mathbf{- 1 3 . 2}$ & $\mathbf{- 6 . 1}$ & $\mathbf{- 1 5 . 8}$ & $\mathbf{- 6 . 1}$ \\
$212-227$ & 15.9 & $\mathbf{- 6 . 0}$ & $\mathbf{- 7 . 9}$ & $\mathbf{- 6 . 3}$ & -1.5 & $\mathbf{- 1 2 . 1}$ & $\mathbf{- 1 0 . 0}$ \\
\hline
\end{tabular}

${ }^{a}$ The $\%$ change for a particular peptide is calculated by Equation 7 (Chapter III). ${ }^{b}$ The maximum number of exchangeable protons within the indicated peptide, assuming $100 \%$ deuteration (Chapter III). A fully deuterated peptide would theoretically have acquired this amount of deuterons. ${ }^{c}$ The deuterium uptake for these peptides was quantified from the non-reducing digests. All other peptides are from the digests with TCEP. When isotopic clusters appear in both the TCEP and non-TCEP digests, the differences in deuteration are negligible when comparing similar peptides. Certain peptides appear exclusively under non-reducing or reducing conditions, hence the necessity of performing both types of peptic digests. ${ }^{d}$ The values in bold represent significant changes in deuteration of greater than $-4.5 \%$. 


\section{$\underline{\text { Discussion }}$}

The binding interface for the $\gamma^{\prime}$ peptide has been localized to thrombin anionbinding exosite II through fluorescence studies $(69,70)$. Additional information is needed on the conformational features the peptide adopts upon binding and the contribution each sulfotyrosine makes toward the thrombin interaction. There is also interest in determining the residues within thrombin ABE-II that make contact with the peptide and the possible long range effects associated with a binding event at this exosite. The current work employs solution NMR methods and HDX coupled with MALDI-TOF mass spectrometry to further describe the $\gamma^{\prime}$ peptide-thrombin relationship (167). Since the $\gamma^{\prime}$ peptide-thrombin structure has been recently crystallized (242), the data in this chapter will be compared to the X-ray structure when relevant to the discussion.

Analysis of NMR Data: Insight into the Structural Requirements for the $\gamma^{\prime}$ Peptide Interaction with Thrombin's ABE-II. One-dimensional (1D) ${ }^{1} \mathrm{H}$ and ${ }^{31} \mathrm{P}$ NMR demonstrate that the $\mathrm{Y}_{\mathrm{P}}^{418} \mathrm{Y}_{\mathrm{P}}^{422}$ peptide makes significant contact with the IIa surface (Figures 20 and 24). The NMR broadening effects are in agreement with observations that truncation of the $\gamma^{\prime}$ peptide from $\mathrm{E}^{411}-\mathrm{L}^{427}$ to $\mathrm{A}^{414}-\mathrm{L}^{427}$ decreases binding affinity almost 4-fold whereas peptides lacking the $\mathrm{N}$-terminal residues $\mathrm{P}^{410}-\mathrm{T}^{416}$ demonstrate no observable binding to ABE-II $(60,69)$. The current work makes it possible to identify individual protons on amino acids that participate in this binding. The consequences of removing the phosphates were also screened using $1 \mathrm{D}{ }^{1} \mathrm{H}$ and ${ }^{31} \mathrm{P}$ NMR line broadening. Figures 21 and 22 demonstrate that both the $\mathrm{Y}_{\mathrm{P}}{ }^{418}$ and $\mathrm{Y}_{\mathrm{P}}^{422}$ peptides are interacting with IIa. The binding segment of the $\mathrm{Y}_{\mathrm{P}}^{422}$ peptide exhibits more extensive contact with IIa than the $\mathrm{Y}_{\mathrm{P}}^{418}$ peptide, suggesting a more instrumental role for $\mathrm{Y}_{\mathrm{P}}^{422}$ in anchoring the $\gamma^{\prime}$ 
peptide segment to thrombin. Although phosphorylation at either position is enough to support the ABE-II interaction, the presence of both phosphates in the $\gamma^{\prime}$ peptide promotes more effective binding to IIa as evidenced by the increased line broadening.

Two-Dimensional (2D) trNOESY spectra supply critical information regarding the conformational features the peptides adopt when interacting with thrombin. All three phosphorylated peptides generate similar spectra illustrating that IIa-bound structural characteristics are preserved as long as one $\mathrm{Y}_{\mathrm{P}}$ is present. Key conformational features for the bound $\gamma^{\prime}$ peptides include a turn between residues $\mathrm{Y}^{422}-\mathrm{D}^{425}$ (Figure 23A), a trans conformation adopted by both Xxx-Pro bonds, and a cluster of NOEs centered around the aromatic rings of both $\mathrm{Y}$ residues (Figure 23B). The $\mathrm{Y}$ residues and their neighbors are major contributors to establishing the bound structure. The results demonstrate the requirement of at least one $Y_{P}$ in the interaction of the $\gamma^{\prime}$ chain and ABE-II.

2D NMR with Gamma-Thrombin: The $\gamma^{\prime}$ Peptide Targets ABE-II. Previous solution studies have demonstrated that the primary binding site for the $\gamma^{\prime}$ peptide is ABE-II $(69,70)$. Recent crystal structures of GpIb $\alpha$ and thrombin display interactions occurring with both ABE-II and ABE-I $(96,97)$. GpIb $\alpha$ and the $\gamma^{\prime}$ peptide have quite a comparable clustering of $Y_{S}$, E, and D residues (Table 1). Therefore, 2D trNOESY NMR spectra were obtained for the $\gamma^{\prime}$ peptide in the presence of gamma-thrombin to address if the peptide is interacting with ABE-I or influenced by this exosite. The gamma variant of thrombin retains catalytic activity while possessing an impaired ABE-I due to cleavages after residues $\mathrm{R}^{75} / \mathrm{R}^{77 \mathrm{~A}}$ and $\mathrm{K}^{149 \mathrm{E}}(53)$. The spectra obtained were remarkably similar to the spectra obtained for wild type thrombin, suggesting that the structural information results from interactions at ABE-II (data not shown). 
Comparing the NMR data with the X-ray Crystal Structure. Before the X-ray structure for this complex was solved, the NMR data predicted several conformational features adopted by the bound peptide. One of these structural elements was a turn conformation at the peptide's C-terminus $\left(\mathrm{Y}_{\mathrm{P}}^{422}-\mathrm{D}^{425}\right)(167)$. The NMR work suggested extensive interactions with every residue within the peptide with the exception of the first three residues $\left(\mathrm{P}^{410}, \mathrm{E}^{411}\right.$, and $\left.\mathrm{H}^{412}\right)$ (167). Figure 29 depicts the crystal structure of the $\gamma^{\prime}$ peptide-IIa (242). The structure was reported at $2.4 \AA$ and is lacking sufficient electron density to model residues $\mathrm{P}^{410}, \mathrm{E}^{411}, \mathrm{H}^{412}$, and $\mathrm{L}^{427}(242)$. The 1D NMR results (Figure 20) predicted a limited role for the $\gamma^{\prime}$ peptide's N-terminus in binding to IIa (167), which is in agreement with the X-ray structure (242) and solution studies with truncated $\gamma^{\prime}$ peptides $(60,69)$. As for $\mathrm{L}^{427}$, the NMR study suggests that this residue does contribute to IIa binding (167), even though $\mathrm{L}^{427}$ is not modeled in the structure (242).

The 2D trNOESY experiments provided evidence for a trans configuration for the two $\mathrm{P}$ amide bonds in the peptide (167). As mentioned, $\mathrm{H}^{412}$ was not modeled in the $\mathrm{X}$ ray structure, thus the $\mathrm{H}^{412}-\mathrm{P}^{413}$ bond is not present (242). However, the $\mathrm{Y}_{\mathrm{P}}^{422}-\mathrm{P}^{423}$ bond is in the trans configuration, forming the beginning of the turn structure that was also predicted by the NMR data (Figures 23A and 29) (242). An additional feature of the 2D trNOESY spectra was the presence of significant C-terminal interactions with the protons spanning residues $\mathrm{Y}_{\mathrm{P}}{ }^{418}-\mathrm{L}^{427}$ (Figures 23B and 29; Appendix B) (167). The C-terminal turn amongst residues $\mathrm{Y}_{\mathrm{P}}^{422}-\mathrm{D}^{425}$ enables the peptide to bend back on itself, supporting the extensive amount of NOEs observed for the aromatic ring of $\mathrm{Y}_{\mathrm{P}}{ }^{422}(242)$. 
A

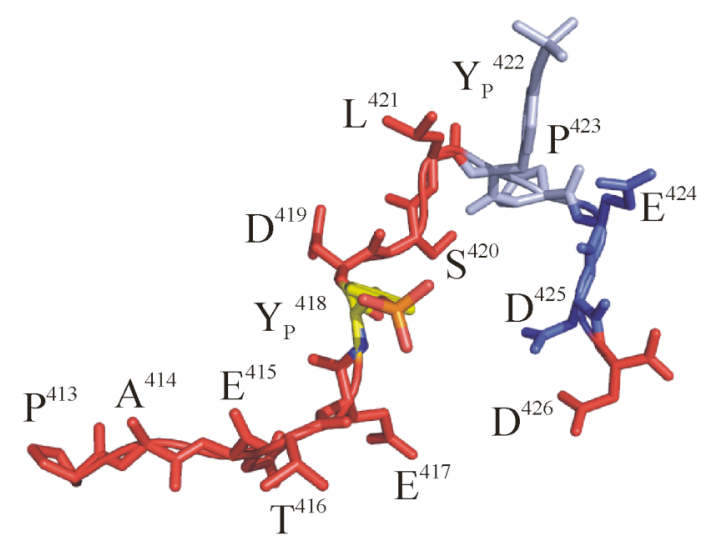

B

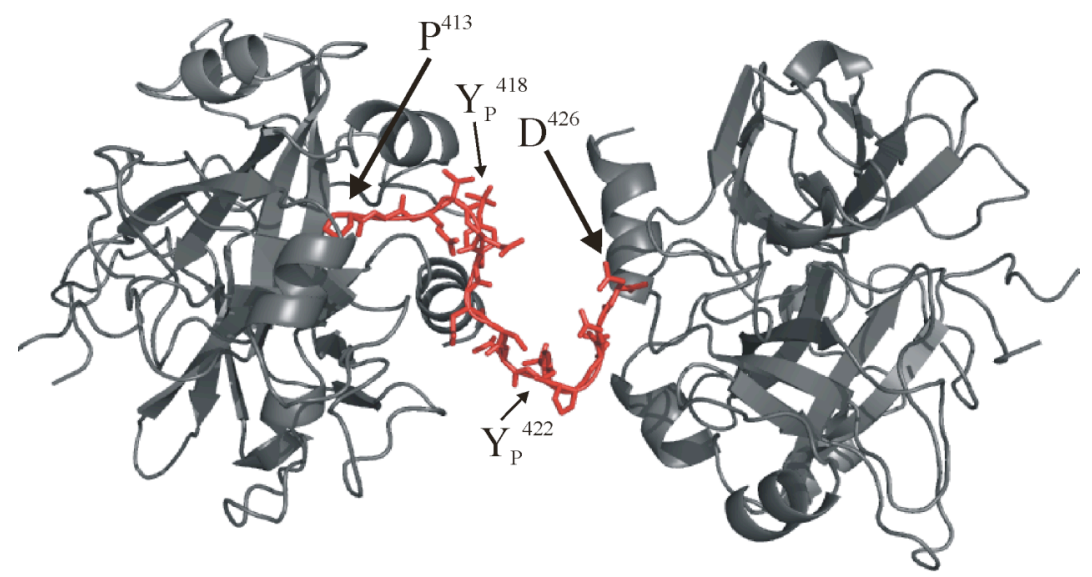

Figure 29: Crystallographic views of the $\gamma^{\prime}$ peptide bound to thrombin (I) (2HWL). A. The $\gamma^{\prime}$ peptide isolated from thrombin. The individual amino acids are labeled. $\mathrm{Y}_{\mathrm{P}}{ }^{418}$ is yellow. The trans bond between $\mathrm{Y}_{\mathrm{P}}{ }^{422}-\mathrm{P}^{423}$ is light blue and the turn structure $\mathrm{Y}_{\mathrm{P}}{ }^{422}-\mathrm{D}^{425}$ is blue. B. Illustration of $\gamma^{\prime}$ peptide induced dimerization of IIa. The turn conformation is situated between the two symmetry related IIa monomers.

Analysis of HDX Results (I): Comparing the HDX data with the X-ray Crystal Structure. HDX coupled with the MALDI-TOF MS was performed to gain further insight on the location of the $\gamma^{\prime}$ peptide interface with IIa. Observed decreases in deuteration could be the product of a restricted ensemble of conformations (protein stabilization), a conformational change, or a ligand-protein interface. When examining the HDX results, differences in deuteration at sites located within ABE-II are due primarily to the shielding of backbone amides from solvent by the $\gamma^{\prime}$ peptide. Regions 
distant from the $\gamma^{\prime}$ peptide-IIa interface that display protection from the solvent are probably experiencing a change in dynamics due to binding at ABE-II.

A number of mutagenesis studies and crystallographic structures have demonstrated the importance of IIa residues $\mathrm{R}^{93}, \mathrm{R}^{101}$, and $\mathrm{K}^{240}$ in the binding of ligands to ABE-II (66, 73, 96, 97, 168, 169, 250) (Figure 19). As described in the introduction, ligand binding to IIa appears to involve two separate ABE-II interfaces that both utilize residues $\mathrm{R}^{93}$, $\mathrm{R}^{101}$, and $\mathrm{K}^{240}$ (Figure 19). The HDX data present evidence that the $\gamma^{\prime}$ peptide is interacting with ABE-II residues within the fragments 85-99 and 173-181 (Table 5). These results compare extremely well to the $\gamma^{\prime}$ peptide-IIa X-ray structure, which illustrates an ABE-II interaction similar to heparin and GpIba (Figures 19 and 30A). Specifically, the HDX data supports the observed electrostatic interactions between $\mathrm{R}^{93 \mathrm{~T}}$ $\mathrm{S}^{420 \gamma^{\prime}}$ and $\mathrm{N}^{179 \mathrm{~T}}-\mathrm{D}^{419 \gamma^{\prime}}$ (Figure 30B). In addition, the residue $\mathrm{R}^{93}$ from a symmetry related IIa interacts with both $\mathrm{D}^{425}$ and $\mathrm{D}^{426}$ (Figure 30B). The authors also describe a hydrophobic pocket formed by $\mathrm{I}^{162}, \mathrm{~V}^{163}, \mathrm{R}^{165}, \mathrm{~F}^{181}$ (part of peptide 173-181), $\mathrm{A}^{183}$, and the disulfide $\mathrm{C}^{168}-\mathrm{C}^{182}$ into which the residues $\mathrm{P}^{413}$ and $\mathrm{A}^{414}$ insert (Figure 30B). Unfortunately, lack of peptic digest coverage for many of the residues that bind the $\gamma^{\prime}$ peptide prevented analysis of their contribution to the interaction, including the Cterminal helix 233-240.

There is evidence that binding of the $\gamma^{\prime}$ peptide to IIa ABE-II reduces the rate at which fibrinopeptide $\mathrm{A}(\mathrm{FpA})$ is released from fibrinogen and the chromogenic substrate S2238 is hydrolyzed (113). Contact of the $\gamma^{\prime}$ peptide with the thrombin regions 85-99 and 173-181 could explain the hindered release of FpA. Crystal structures of FpA (7-16) and IIa demonstrate the importance of $\mathrm{R}^{97}, \mathrm{E}^{97 \mathrm{~A}}$ and $\mathrm{I}^{174}$ in orienting FpA in the active 


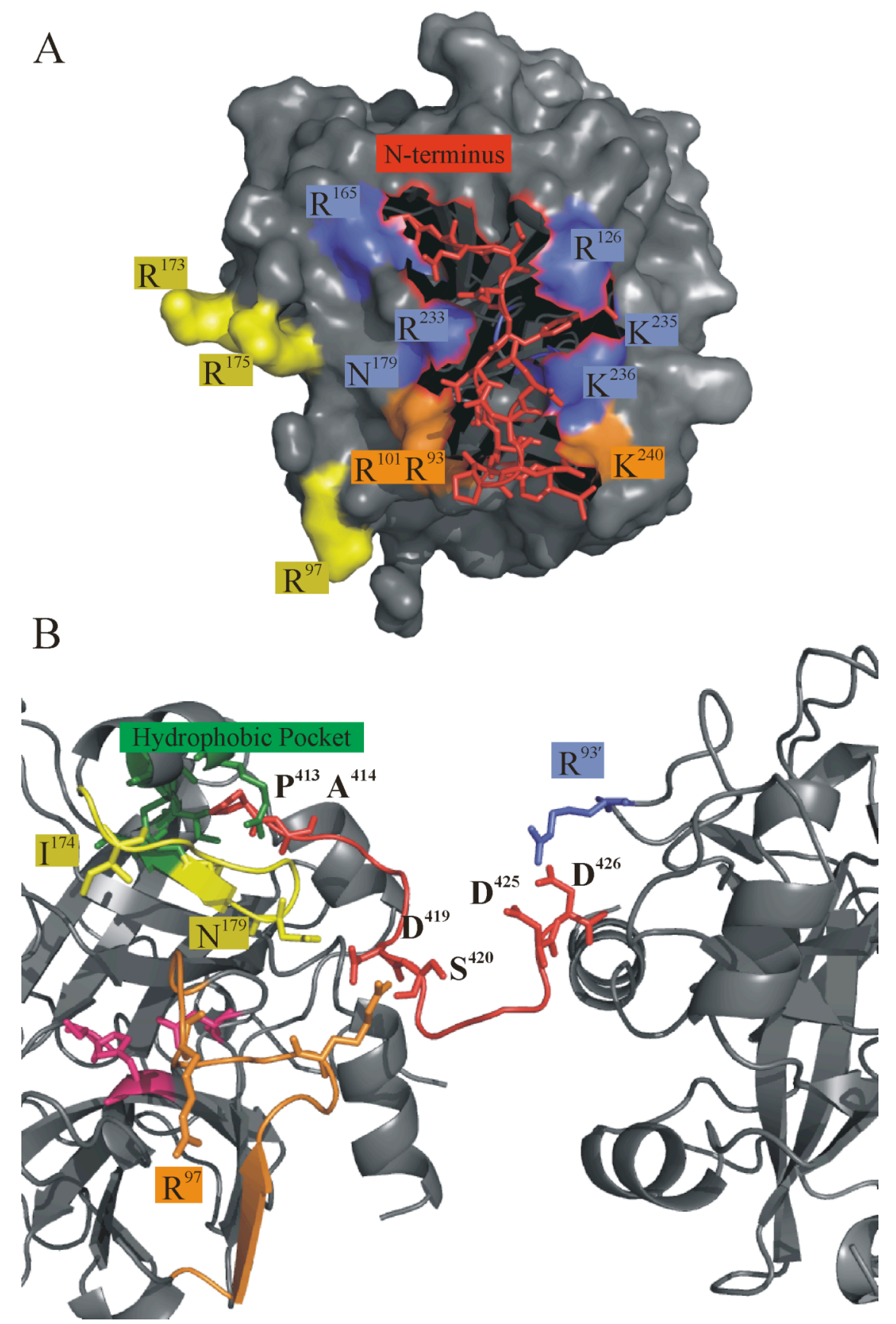

Figure 30: Crystallographic views of the $\gamma^{\prime}$ peptide bound to IIa (II) (2HWL). A. View of the $\gamma^{\prime}$ peptide binding into the groove formed by ABE-II residues in a similar manner as heparin and GpIba. B. Depiction of key interactions detected by HDX and illustrated in the crystal structure. The orange fragment is 85-99 and the yellow fragment is 173-181. The catalytic triad is pink. The text contains descriptions of the relevant features highlighted in this figure including the hydrophobic pocket (green) and the $\gamma^{\prime}$ peptide (red).

site (248). HDX results indicate that regions containing these residues are protected from deuteration in the presence of $\gamma^{\prime}$ peptide. Since the crystal structure has identified the residues $\mathrm{R}^{93}, \mathrm{~N}^{179}$, and $\mathrm{F}^{181}$ as being involved in binding, perhaps the $\gamma^{\prime}$ chain could be 
hindering the helical turn of FpA from optimally accessing the active site region through an allosteric mechanism. The conformation of $\mathrm{R}^{97}, \mathrm{E}^{97 \mathrm{~A}}$ and $\mathrm{I}^{174}$ may be perturbed due to allostery resulting from $\gamma^{\prime}$ peptide binding, ultimately reducing the rate of catalysis ( 70 , 170) (Figure 30B). A similar suggestion has been offered for the inhibition of fibrinogen clotting activity by the chondroitin sulfate moiety of thrombomodulin (250).

Analysis of HDX Results (II): Determining the Consequences of Ligand Binding to ABE-II on Several Key Regions of Thrombin. Binding of ligands (fibrinogen A $\alpha$, PAR-1, thrombomodulin, and hirudin) to ABE-I has clearly been shown to affect reactivity within the thrombin active site region (240). The reduced release of FpA from fibrinogen in the presence of $\gamma^{\prime}$ peptide-IIa (113) supports a linkage from ABE-II to the active site. Conflicting reports address the existence of an allosteric linkage between ABE-I and ABE-II $(170,171)$. The current studies provide evidence that ligand binding to ABE-II is perturbing the rate of HDX at other distant sites. The HDX results suggest lines of communication from ABE-II to ABE-I (Figure 31C), from ABE-II to a segment containing $\mathrm{W}^{215}$ (Figure 31D), and from ABE-II to a region of the A-chain (Figure 31D).

Interaction of the $\gamma^{\prime}$ peptide with ABE-II resulted in a segment of ABE-I representing residues $65-84$ to become more protected from solvent. A pathway from ABE-II to ABE-I can be traced from $\mathrm{L}^{99}$ back toward residue $\mathrm{L}^{65}$ as seen in Figure 31B. Electrostatic interactions between two anti-parallel $\beta$-strands, including contacts between the residues $\mathrm{E}^{86}-\mathrm{K}^{107}, \mathrm{~K}^{87}-\mathrm{K}^{107}$, and $\mathrm{M}^{84}-\mathrm{K}^{109}$ explain how an ABE-II binding event could be relayed to the peripheral ABE-I region involving residues 106-113 (51) (Figure 31C). A similar line of communication has been reported in a prior (233) and in our own HDX study of PPACK inhibited thrombin. PPACK alone offers little protection for residues 
85-96 (Table 5), while the observed protection within 97-99 is in part attributable to contacts with $\mathrm{L}^{99}$. These PPACK interactions are proposed to be transmitted to ABE-I via the $\beta$-strand formed by residues $85-96$ (233)
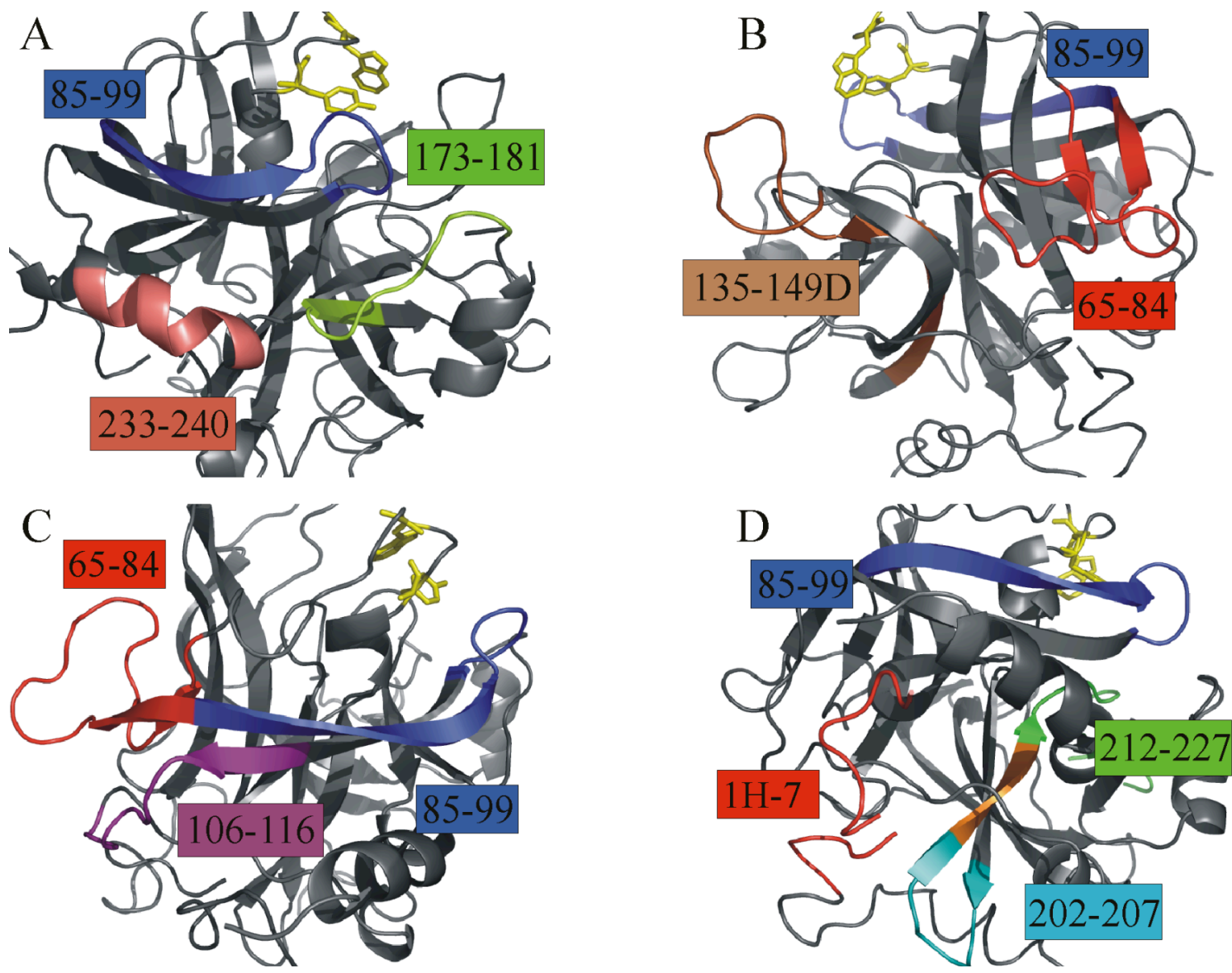

Figure 31: Correlating HDX results with X-ray structure of PPACK-IIa (1PPB). $\mathrm{W}^{60 \mathrm{D}}$ is yellow. A. ABE-II region 85-99 blue and 173-181 green. The additional ABE-II region not observed in the present HDX study, 233-240, is in pink. B. The flexible solvent exposed regions 65-84 red and 135149D brown. Both PPACK and the $\gamma^{\prime}$ peptide significantly stabilize the segments upon ternary complex formation with thrombin. C. Line of communication from ABE-II, 85-99 blue, to ABE-I, 65-84 red and 106-116 purple. D. The A-chain. There are several electrostatic contacts between the A-chain residues $1 \mathrm{H}-7$ red and the B chain residues $202-207$ teal. Also represented are $85-99$ blue and 212-227 green. The $\beta$-strand connecting 202-207 to 212-227 is orange.

Examining the $\gamma^{\prime}$ peptide-PPACK-IIa complex reveals more information about sites of distant influence. The ternary interactions resulted in a synergistic effect on the rate of deuterium exchange for the solvent exposed regions 65-84 (ABE-I) and 135-149D (autolysis loop) (Figure 31B). This suggests that simultaneous binding of $\gamma^{\prime}$ peptide and 
PPACK to thrombin significantly stabilizes these portions of the protease. Effects on the environment of 212-227 are also important to consider. The additive effect observed for the ternary complex suggests that the two ligands may be independently influencing the solvent accessibility of 212-227. The $\gamma^{\prime}$ peptide could be affecting residues near $\mathrm{W}^{215}$ and/or other regions within the residues 212-227, while the D-Phe of PPACK is known to exhibit beneficial interactions with $\mathrm{W}^{215}$ via a perpendicular aryl-aryl arrangement (51).

In addition to altering the deuterium exchange dynamics for regions involving ABE-II, ABE-I, and the active site region, the $\gamma^{\prime}$ peptide also exhibits effects on a portion of the thrombin A chain and a nearby B-chain fragment 202-207 (Figure 31D). As can be seen in Figure 31D, a direct connection cannot be made from ABE-II to the A-chain. Deletion of $\mathrm{K}^{9}$ from the A-chain has recently been shown to perturb the pKa of the catalytic residue $\mathrm{H}^{57}$ slowing the catalytic activity of IIa $(251) . \mathrm{K}^{9}$ contacts $\mathrm{D}^{1 \mathrm{~A}}$ which is part of a segment that undergoes protection from solvent in the presence of the $\gamma^{\prime}$ peptide. The $\gamma^{\prime}$ peptide decreases $k_{\text {cat }}$ values for the hydrolysis of the small chromogenic substrate S2238 (113). Perhaps $\gamma^{\prime}$ peptide binding to ABE-II leads to a line of communication to the A-chain and the nearby B-chain segment 202-207 through the active site.

HDX studies on the binding of ligands to thrombin have revealed both local and long range effects $(172,173,230,233)$. The current work with the $\gamma^{\prime}$ peptide illustrate lines of communication from ABE-II to ABE-I and from ABE-II to regions of the extended active site. Studies with PPACK-IIa display connections from the active site to similar regions of ABE-I and the edge of ABE-II (233). Binding of the thrombomodulin fragment TMEGF45 to thrombin ABE-I has led to perturbations in deuterium exchange within the 90s loop of ABE II $(172,173)$. A distinct linkage between ABE-I and ABE-II 
may also have been demonstrated in earlier work involving a mixture of $\gamma^{\prime}$ peptide, IIa, and Fibrin 1 (fibrinogen lacking the $\gamma^{\prime}$ extension) (60). These different observations support the concept of an inter-exosite linkage reported between the two ABE-II binding ligands prothrombin fragment 2 and $\gamma^{\prime}$ peptide and the ABE-I $(70,170)$. On the contrary, another study with hirudin and heparin ligands did not observe as significant a negative allostery for the ABE-I/ABE-II connections (171). The HDX studies presented here cannot evaluate the extent to which inter-exosite linkages affect enzyme function but they can establish potential lines of communication between the sites.

Conclusions. In this investigation, interactions between a series of $\gamma^{\prime}$ peptides and thrombin were characterized using solution NMR and HDX coupled with MALDI-TOF MS. 1D NMR results indicate that residues $\mathrm{H}^{412}-\mathrm{L}^{427}$ of the $\mathrm{Y}_{\mathrm{P}}^{418} \mathrm{Y}_{\mathrm{P}}^{422}$ peptide are responsible for the primary sites of contact with the thrombin surface and illustrate the importance of sulfonation at the $\mathrm{Y}^{422}$ position. Data from the $2 \mathrm{D}$ trNOESY spectra demonstrate that the ABE-II bound $\gamma^{\prime}$ peptide adopts significant secondary structure in the presence of at least one phosphotyrosine. HDX results suggest that the $\gamma^{\prime}$ peptide interacts with thrombin ABE-II. In response to this binding, other regions of thrombin also experience protection from deuteration including ABE-I, regions near the active site, and a portion of the A-chain. Finally, thrombin can form a ternary complex with the $\gamma^{\prime}$ peptide and PPACK generating an enzyme that is further stabilized from HDX. 


\section{CHAPTER V}

\section{STUDYING GPIB $\alpha$ (269-286) PEPTIDE INTERACTIONS WITH THE THROMBIN ANION BINDING EXOSITES}

\section{Introduction}

GpIb $\alpha$ is part of a transmembrane receptor complex of glycoproteins on the surface of platelets (74-77). GpIb $\alpha$ serves a multitude of functions that include binding coagulation proteins, adhering platelets to the site of damaged tissue, and aiding in the recruitment of leukocytes to combat infection. IIa interacts with GpIbo leading to enhancements in the activation of PAR-1, FXI, and $\operatorname{GpV}(67,83,87)$. As mentioned in Chapter I, GpIb $\alpha$ residues 269-286 share a similar clustering of anionic residues as found in the $\gamma^{\prime}$ peptide (410-427) (Table 1). The studies on $\gamma^{\prime}$ peptide binding to IIa described in Chapter IV were originally conducted due to the absence of a reported crystal structure illustrating this interaction (167). In the case of GpIbo-IIa, two independent crystal structures have been published pertaining to the binding of these proteins $(96,97)$. An unresolved issue is why independent groups attribute GpIb $\alpha$ binding either to ABE-II $(68,92)$ or to ABE-I $(93,101)$, while the $\gamma^{\prime}$ peptide targets ABE-II $(69,70,167,242)$.

The X-ray structures involving GpIb $\alpha$ possess important similarities and differences regarding the mode of IIa interaction $(96,97)$ (Figure 32). In the reported structures, insufficient electron density prevented the modeling of GpIbo's C-terminus. The Dumas structure (1P8V) ends at $\mathrm{Y}^{279}$ (96), while the Celikel structure (1OOK) 
includes 5 additional residues $\left(\mathrm{P}^{280}-\mathrm{T}^{284}\right)$ at the $\mathrm{C}$-terminus (97). The $\mathrm{N}$-terminus of residues 269-286 appears to be rather flexible, adopting a turn conformation in 1P8V (96) and a fully extended structure in 1OOK (97). Though both groups report a 1:1 asymmetric unit, the proteins in symmetry related molecules display unique binding interfaces. Both structures illustrate similar GpIbo interactions with ABE-II, but differ in regard to the depiction of GpIb $\alpha$ binding to ABE-I. 1OOK shows the C-terminal tail of GpIb $\alpha$ potentially dimerizing IIa through interactions with both exosites (97). In 1P8V, the second IIa molecule binds to GpIb $\alpha$ at the C-terminal end of the leucine rich repeat domain (in blue) through ABE-I (96).
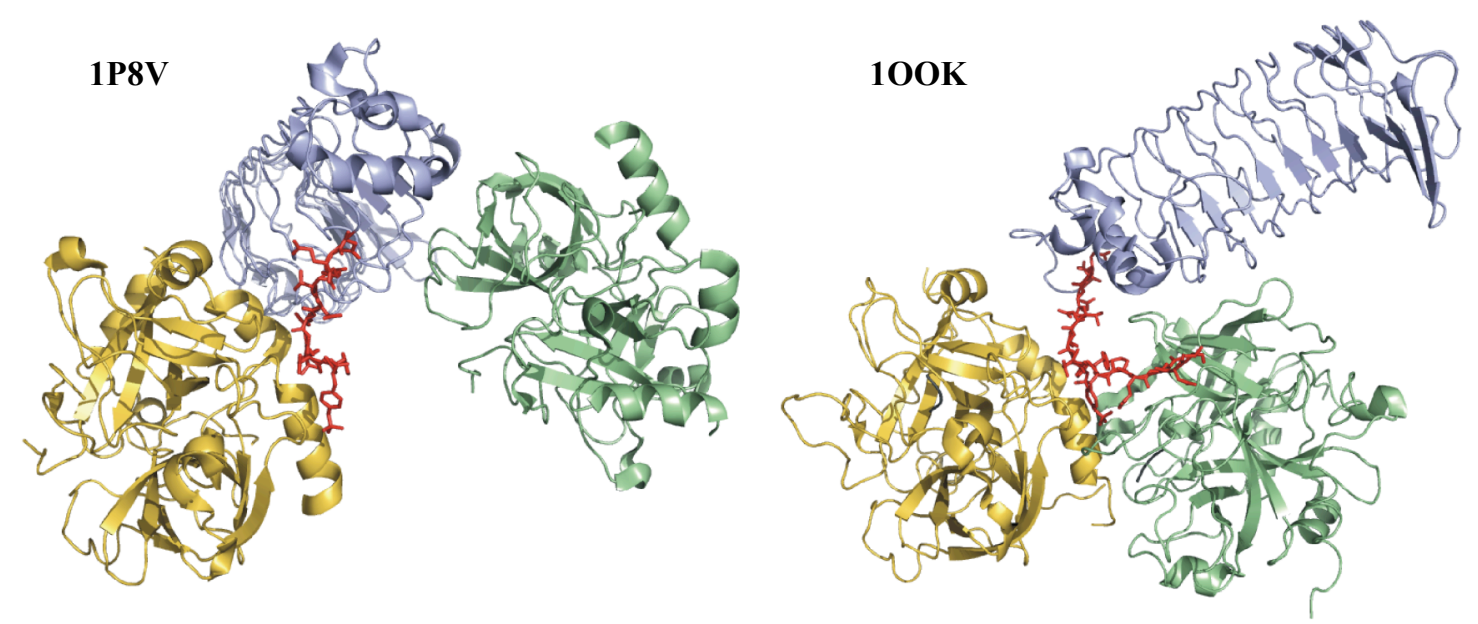

Figure 32: Two views of GpIb $\alpha$ binding to thrombin. The crystal structures depict similar ABE-II interactions but differ in the depiction of thrombin dimerization. In blue is GpIbo and the anionic Cterminal tail is in red. Thrombin in gold binds to the C-terminal tail, while thrombin in green binds at different interfaces. The structure on the left is 1P8V (96), the structure on the right 1OOK (97).

The focus of this chapter is to further characterize the GpIb $\alpha$-IIa interaction by using peptides based on the sequence 269-286 (Table 6), perhaps the $\gamma^{\prime}$ peptide and the GpIb $\alpha$ peptide share similar characteristics when binding thrombin. For example, the Cterminal turn structure observed for the $\gamma^{\prime}$ peptide $(167,242)$ is not being modeled in the Dumas structure (1P8V), which ends at residue $\mathrm{Y}^{279}(96)$. The sequence of the $\gamma^{\prime}$ peptide 
turn $\left.{ }^{422} \mathrm{Y}_{\mathrm{S}} \mathrm{PED}^{425}\right)$, however, is very similar to the comparable C-terminal stretch of residues in GpIb $\alpha\left({ }^{279} \mathrm{Y}_{\mathrm{S}} \mathrm{PEE}^{282}\right.$ ) (Table 1). In addition, when the GpIb $\alpha$ peptide binds to IIa there are comparable long-range allosteric effects observed as with the $\gamma^{\prime}$ peptide. For the GpIba studies (as with the $\gamma^{\prime}$ peptide), phosphotyrosines were substituted for the naturally occurring sulfotyrosines without sacrificing binding affinity. This type of substitution has been reported to have a minimal effect on binding affinity $(69,241)$.

1D and 2D NMR experiments demonstrate that the GpIb $\alpha$ peptide binds to IIa in an extended conformation, with a majority of the residues contacting IIa's surface. Additional work with HDX coupled with MALDI-TOF MS suggests a concentration dependence in regard to the GpIba binding interface on IIa. In addition, the HDX results display allostery related to GpIb $\alpha$ interactions with IIa. GpIb $\alpha$ peptide binding to IIa alters the dynamics of deuterium incorporation at sites unaffiliated with the binding interface. Finally, analytical ultracentrifugation (AUC) was performed to observe the behavior of thrombin when binding the GpIba peptide or the $\gamma^{\prime}$ peptide in solution.

\section{Materials and Methods}

Synthetic Peptides and Thrombin Purification. Bachem (King of Prussia, PA) synthesized peptides based on residues 269-286 of GpIba (Table 6). SynPep (Dublin, CA) synthesized a peptide based on residues 410-427 of the human fibrinogen $\gamma^{\prime}$ chain variant (Table 3 in Chapter IV, only the $\mathrm{Y}_{\mathrm{P}}^{418} \mathrm{Y}_{\mathrm{P}}^{422}$ peptide). MALDI-TOF MS measurements on an Applied Biosystems Voyager DE-Pro mass spectrometer were used to verify peptide $\mathrm{m} / \mathrm{z}$ values. The concentrations of the peptide in solution were determined by quantitative amino acid analysis (AAA Service Laboratory, Boring, OR). 
Bovine IIa was purified as described by Trumbo and Maurer (243). The use of bovine IIa in place of human IIa is justified in the Material and Methods of Chapter IV.

\begin{tabular}{|c|c|}
\hline $\mathbf{Y}_{\mathbf{P}}{ }^{276} \mathbf{Y}_{\mathbf{P}}{ }^{278} \mathbf{Y}_{\mathbf{P}}{ }^{279}$ & ${ }^{269}$ DEGDTDL $_{\mathbf{P}}$ DY $\mathbf{Y}_{\mathbf{P}} \mathbf{Y}_{\mathbf{P}}$ EEDTEG $^{286}$ \\
\hline $\mathbf{Y}_{\mathbf{P}}{ }^{276} \mathbf{Y}^{278} \mathbf{Y}_{\mathbf{P}}{ }^{279}$ & ${ }^{269}$ DEGDTDLY $_{\mathbf{P}}$ DYY $\mathbf{Y}_{\mathbf{P}} \mathrm{EEDTEG}^{286}$ \\
\hline $\mathrm{Y}^{276} \mathrm{Y}^{278} \mathrm{Y}^{279}$ & ${ }^{269}$ DEGDTDLYDYYEEDTEG ${ }^{286}$ \\
\hline
\end{tabular}

${ }^{1} H$ and ${ }^{31} P$ NMR Sample Preparation and Analysis. Approximately a 1:10 ratio of enzyme to peptide was maintained for the complex studied. Sample preparation and analysis were similar to those described by Trumbo and Maurer (190). Selective features are summarized here. Each $400 \mu \mathrm{l}$ NMR sample contained $1.5 \mathrm{mM}$ peptide and either zero or $150 \mu \mathrm{M}$ bovine IIa. The samples were buffered in $25 \mathrm{mM} \mathrm{H}_{3} \mathrm{PO}_{4}, 150 \mathrm{mM} \mathrm{NaCl}$, $0.2 \mathrm{mM}$ EDTA, $\mathrm{pH} 5.6$ and $10 \% \mathrm{D}_{2} \mathrm{O} .{ }^{1} \mathrm{H}$ NMR experiments were performed on a Varian Inova $500 \mathrm{MHz}$ equipped with a triple resonance probe and pulsed-field z-axis gradients. 2D transferred NOESY experiments of the enzyme-peptide complexes were run at $17{ }^{\circ} \mathrm{C}$ with 32 transients, $512 t_{1}$ increments, mixing time of $400 \mathrm{~ms}$, and a spectral width of $5006 \mathrm{~Hz}$. Spectra were processed using FELIX2000 software (Accelerys, San Diego, CA) on a Silicon Graphics $\mathrm{O}_{2}$ workstation. For ${ }^{31} \mathrm{P}$ NMR, spectra were recorded on a Varian Inova $500 \mathrm{MHz}$ equipped with a $5 \mathrm{~mm}$ broadband probe tuned to $202.4 \mathrm{MHz}$. The spectra were referenced to the peak corresponding to $\mathrm{H}_{3} \mathrm{PO}_{4}(0.00 \mathrm{ppm})$.

HDX Sample Preparation. Thrombin was buffer exchanged into $75 \mathrm{mM} \mathrm{NaCl}$, $12.5 \mathrm{mM} \mathrm{NaH} \mathrm{PO}_{4}, \mathrm{pH} 6.5$ with an Amicon-Ultra 4 unit (10 kDa MWCO). The concentration of IIa for all HDX preparations varied between 20-25 $\mu \mathrm{M}$. Enough GpIb $\alpha$ peptide was added to achieve a 20:1 or 40:1 ratio during the HDX experiments. For the $\gamma^{\prime}$ peptide, only 40:1 ratios were examined during this investigation. The data reported 
for IIa alone and $\gamma^{\prime}$ peptide-IIa (20:1) was previously published and discussed in Chapter IV (167); inclusion in this chapter is purely for comparison to GpIba results. The aliquots of buffer-exchanged IIa with peptide were evaporated to dryness using a SpeedVac unit (Savant). The dry aliquots were stored at $-70^{\circ} \mathrm{C}$.

HDX Experiments. Dry aliquots of $\gamma^{\prime}$ peptide-IIa (40:1) or GpIb $\alpha$-IIa (20:1 and 40:1) were allowed to come to room temperature before beginning the experiment. First, $12 \mu \mathrm{L}$ of $\mathrm{D}_{2} \mathrm{O}$ was added to the dry aliquot yielding the final concentrations: $40-50 \mu \mathrm{M}$

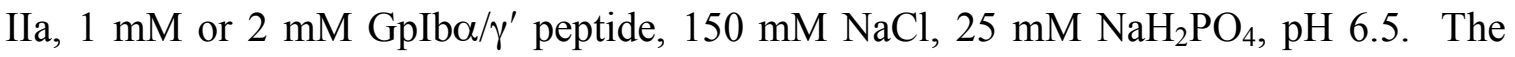
samples were incubated in a desiccator at room temperature for 1 minute or 10 minutes. After incubation, $114 \mu \mathrm{L}$ of $0.1 \%$ TFA pH 2.5 (on ice) was added to quench the solution. Next, $66 \mu \mathrm{L}$ of the quenched solution was transferred to a tube of activated pepsin bound to agarose. To the remaining $60 \mu \mathrm{L}, 6 \mu \mathrm{L}$ of $1 \mathrm{M} \mathrm{TCEP}-\mathrm{HCl}$ in $0.1 \%$ TFA was added to reduce the disulfides bonds of IIa and then immediately added to another tube of pepsin. Digestion occurred on ice for 10 minutes. Centrifugation at $4{ }^{\circ} \mathrm{C}$ separated the digest from the pepsin and $8.2 \mu \mathrm{L}$ aliquots were immediately frozen in liquid $\mathrm{N}_{2}$.

HDX Analysis. A frozen aliquot was thawed, immediately mixed with an equal volume of $10 \mathrm{mg} / \mathrm{mL} \alpha$-CHCA matrix in 1:1:1 ethanol/acetonitrile/0.1\% TFA, $\mathrm{pH} 2.2$ and $0.5 \mu \mathrm{L}$ spotted onto a chilled stainless steel MALDI plate. The plate was dried with the SpeedVac (Savant) and inserted into the MALDI-TOF MS. This procedure should consume around five minutes of time, limiting the amount of hydrogen back exchange. The spectra were collected in the reflector mode over a mass range of $800-3500 \mathrm{~m} / \mathrm{z}$ with 256 laser shots per spectrum. Peptides obtained from the peptic digest of thrombin were previously identified $(167,233,234)$. 
The spectra were collected and analyzed using Data Explorer (Applied Biosystems). Calibration of the spectra involved two reference peptides, one with the singly protonated monoisotopic mass of $888.4943 \mathrm{Da}(46-52)$ and the other with the singly protonated pentaisotopic mass of 2106.1505 $\mathrm{Da}(85-99)$. The amount of deuterium uptake by each peptide and the $\%$ difference relative to IIa alone was quantified as described by Sabo et $a l$. and in Chapter III (167). At least three spectra were collected during each trial and three trials were run for each time point under each condition.

Analytical Ultracentrifugation. IIa was inhibited with a two-fold excess of PPACK for $30 \mathrm{~min}$. An assay involving the chromogenic substrate S2238 was employed to ensure that IIa was inactive. PPACK-IIa was then subjected to overnight dialysis with $150 \mathrm{mM} \mathrm{NaCl}, 25 \mathrm{mM}$ phosphate, $\mathrm{pH}$ 7.4. The $\gamma^{\prime}$ or GpIba peptide was lyophilized and reconstituted in the same buffer. A series of sedimentation velocity AUC experiments ranging from $1,2,5,10$, and 20 times peptide to IIa $(4.5-7.0 \mu \mathrm{M})$ in duplicate was performed in a XL-A AUC (Beckman-Coulter). The parameters for each run were $60,000 \mathrm{rpm}, 25^{\circ} \mathrm{C}$, and $280 \mathrm{~nm}$. The data was analyzed with the program DC-DT+.

\section{$\underline{\text { Results }}$}

${ }^{1} H$ and ${ }^{31} P$ One-Dimensional Line Broadening NMR. 1D NMR experiments were performed to determine which proton or phosphorous nuclei within the GpIb $\alpha$ (269-286) peptide interact with IIa. The first set of spectra collected examined GpIb $\alpha$ $\left(\mathrm{Y}_{\mathrm{P}}{ }^{276} \mathrm{Y}_{\mathrm{P}}{ }^{278} \mathrm{Y}_{\mathrm{P}}{ }^{279}\right)$ and displayed the absence of significant line broadening for the ${ }^{1} \mathrm{H}$ 1D NMR spectra of the peptide in the presence of IIa (data not shown). Similar results were obtained when performing ${ }^{31} \mathrm{P}$ 1D NMR (data not shown). These findings indicate that the triply phosphorylated peptide binds too tightly to IIa's surface. Thus, the transfer of 
information regarding peptide proton or phosphorous interactions with the protein back to the free peptide population does not occur. The result is a $1 \mathrm{D}$ spectrum for the peptide in the presence of IIa similar to the free peptide (see Chapter II for further explanation).

Since the GpIb $\alpha\left(\mathrm{Y}_{\mathrm{P}}{ }^{276} \mathrm{Y}_{\mathrm{P}}{ }^{278} \mathrm{Y}_{\mathrm{P}}{ }^{279}\right)$ peptide spectra did not display line broadening, a doubly phosphorylated variant was synthesized. The GpIba $\left(\mathrm{Y}_{\mathrm{P}}{ }^{276} \mathrm{Y}_{\mathrm{P}}{ }^{279}\right)$ peptide still interacts with IIa (demonstrated by the two crystal structures $(96,97))$, but the absence of one phosphotyrosine may sufficiently weaken the interaction for the appearance of $1 \mathrm{D}$ line broadening. The spectra in Figure 33 focus on the amide region and do illustrate line broadening in the presence of IIa. Most of the broadening encompasses residues $\mathrm{D}^{275}$ $Y_{P}{ }^{279}$, while a modest amount of broadening is observed for residues $E^{281}-D^{283}$. However, the line broadening is not extensive, with residues $\mathrm{D}^{269}-\mathrm{D}^{274}$ and $\mathrm{T}^{284}-\mathrm{G}^{286}$ appearing unchanged from the free peptide spectrum. Curiously, the ${ }^{31} \mathrm{P}$ spectra do not present evidence of line broadening (data not shown). These results suggest two possibilities. The peptide may be binding too tightly for the $\mathrm{N}$ - and $\mathrm{C}$-terminal residues to display line broadening or these residues are not involved in the interaction with IIa.

A final peptide was synthesized without any phosphotyrosines. Much more line broadening is evident with the unphosphorylated peptide (Figure 33). The central portion of the peptide spanning $\mathrm{D}^{274}-\mathrm{Y}^{279}, \mathrm{E}^{281}$, and $\mathrm{D}^{283}$ display a significant amount of line broadening. The residues $E^{282}$ and $E^{285}$ show a modest amount of broadening. Finally, the $\mathrm{N}$-terminal residues $\mathrm{D}^{269}-\mathrm{T}^{273}$ and the $\mathrm{C}$-terminal residues $\mathrm{T}^{284}$ and $\mathrm{G}^{286}$ do not appear to be essential for the interaction of GpIb $\alpha$ (269-286) with IIa. 

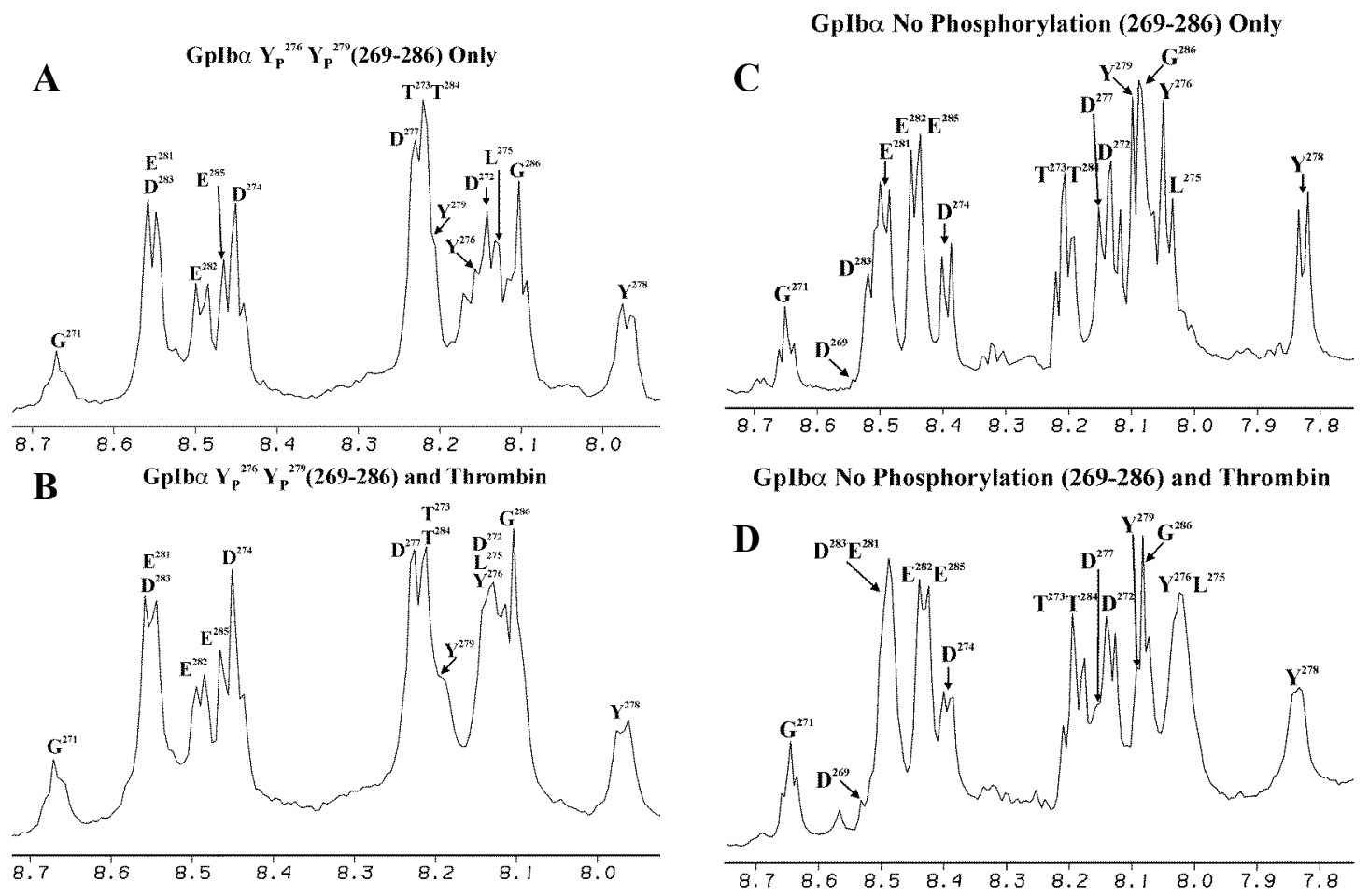

Figure 33: Line broadening spectra for the GpIb $\alpha$ peptides in the presence of thrombin. All NMR samples are in $25 \mathrm{mM} \mathrm{H}_{3} \mathrm{PO}_{4}, 150 \mathrm{mM} \mathrm{NaCl}, 200 \mu \mathrm{M}$ EDTA, and $10 \% \mathrm{D}_{2} \mathrm{O}, \mathrm{pH}$ 5.6. A. 1D NMR spectrum for $1.5 \mathrm{mM} \mathrm{Y}_{\mathrm{P}}{ }^{276} \mathrm{Y}_{\mathrm{P}}{ }^{279}$ peptide in solution. B. 1D NMR spectrum for $1.5 \mathrm{mM} \mathrm{Y}_{\mathrm{P}}{ }^{276} \mathrm{Y}_{\mathrm{P}}{ }^{279}$ peptide in the presence of $0.148 \mathrm{mM}$ IIa. The amide protons that display the most line broadening are $\mathrm{D}^{275}-\mathrm{Y}_{\mathrm{P}}^{279}$. A moderate amount of line broadening is evident for the NHs of $E^{281}-D^{283}$. Line broadening is not observed for the amide protons of $\mathrm{D}^{269}-\mathrm{D}^{274}$ and $\mathrm{T}^{284}-\mathrm{G}^{286}$. C. 1D NMR spectrum for $1.5 \mathrm{mM}$ unphosphorylated peptide in solution. D. 1D NMR spectrum for $1.5 \mathrm{mM}$ unphosphorylated peptide in the presence of $0.150 \mathrm{mM}$ IIa. The amide protons that display the most line broadening are $\mathrm{D}^{274}-\mathrm{Y}_{\mathrm{P}}^{279}, \mathrm{E}^{281}$, and $\mathrm{D}^{283}$. A moderate amount of line broadening is evident for the $\mathrm{NHs}$ of $\mathrm{E}^{282}$ and $\mathrm{E}^{285}$. Line broadening is not observed for the amide protons of $\mathrm{D}^{269}-\mathrm{T}^{273}, \mathrm{~T}^{284}$, and $\mathrm{G}^{286}$.

Two-Dimensional Transferred Nuclear Overhauser Effect NMR. For the triply phosphorylated peptide, the 2D spectra of the free peptide were indistinguishable from the spectrum with the peptide in the presence of the enzyme (data not shown). For the doubly phosphorylated and unphosphorylated GpIb $\alpha$ peptides, the 2D trNOESY spectra displayed only nearest neighbor NOEs in the presence of the enzyme. Appendix A has tables for the chemical shift assignments of both the free and bound unphosphorylated GpIba. Only 2D NMR spectra in the presence of thrombin were collected for GpIb $\alpha$ $\left(\mathrm{Y}_{\mathrm{P}}^{276} \mathrm{Y}_{\mathrm{P}}^{279}\right)$ and these resonances are compiled in Appendix A. 


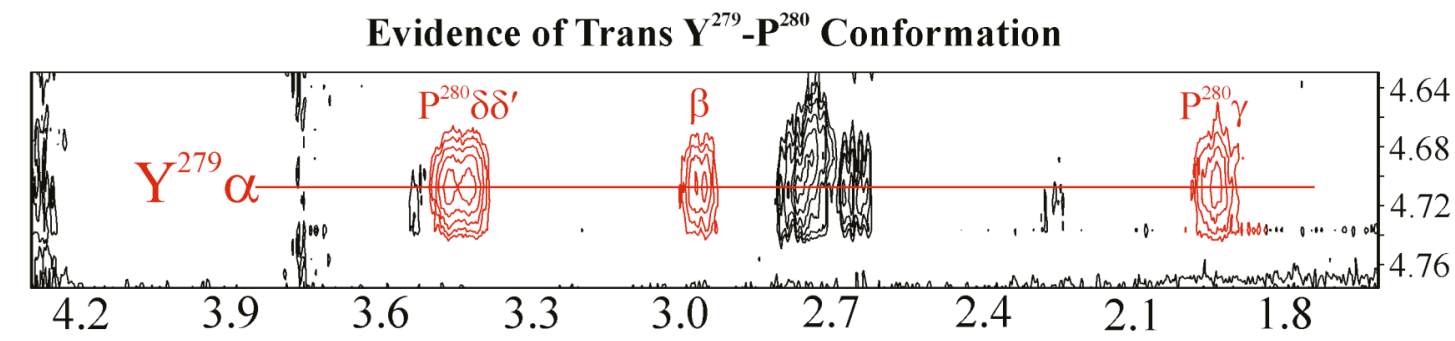

Fingerprint Region: Amide to Aliphatic Protons

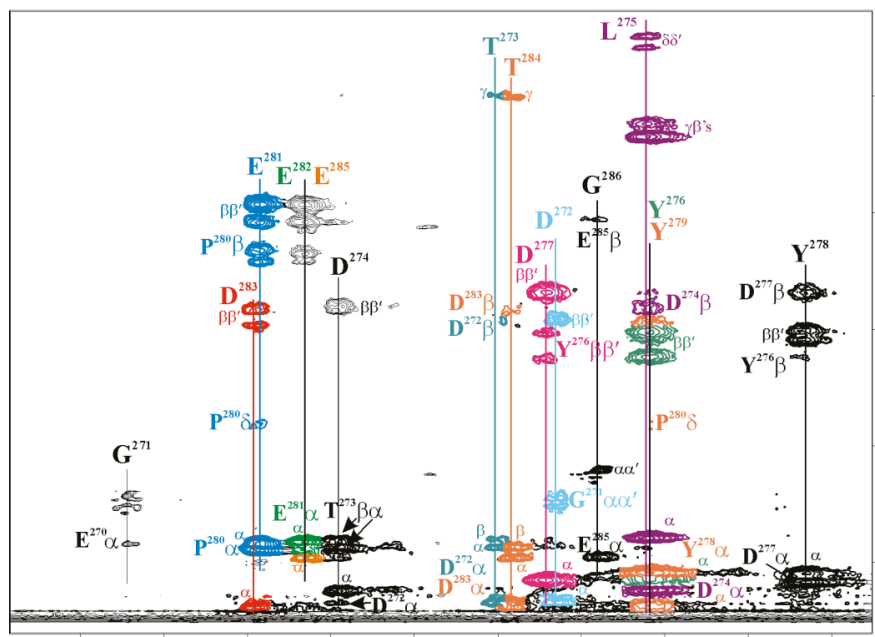

Amide-Amide Region

8.7

8.5

8.3

8.1

7.9

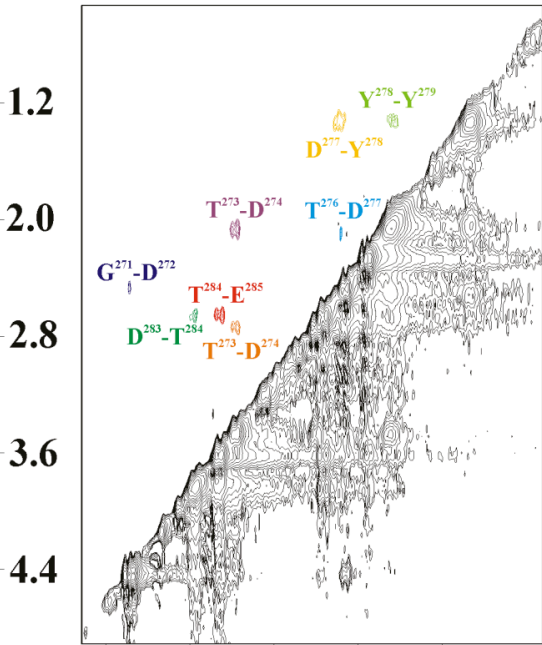

7.7

7.9

8.1

8.3

Figure 34: Representative spectra of GpIba peptide NOEs in the presence of thrombin. 2D trNOESY spectrum of the unphosphorylated GpIba $(1.5 \mathrm{mM})$ bound to IIa $(0.150 \mathrm{mM})$. The top panel illustrates the evidence for a trans $\mathrm{Y}^{279}-\mathrm{P}^{280}$ peptide bond. The bottom panels demonstrate that GpIb $\alpha$ exists in an extended structure when bound to IIa due to the presence of only nearest neighbor NOEs. The NOEs are color coded according to the corresponding residue.

Figure 34 presents some of the key features from the unphosphorylated GpIb $\alpha$-IIa 2D trNOESY spectra. The presence of only nearest neighbor NOEs suggests that the peptide adopts an extended conformation when binding to IIa. In addition, the $\mathrm{Y}^{279}-\mathrm{P}^{280}$ amide bond is in the trans conformation. With the GpIb $\alpha\left(\mathrm{Y}_{\mathrm{P}}^{276} \mathrm{Y}_{\mathrm{P}}{ }^{279}\right)$ peptide, slightly fewer inter-residue NOEs are observed in the presence of IIa when compared to unphosphorylated GpIba (data not shown). With the doubly phosphorylated GpIba, a trans conformation is also evident for the $\mathrm{Y}_{\mathrm{P}}{ }^{279}-\mathrm{P}^{280}$ peptide bond. 
HDX Experiments (I): Observing the Effect of the GpIb $\alpha\left(Y_{P}{ }^{276} Y_{P}{ }^{278} Y_{P}{ }^{279}\right)$ Peptide on Thrombin's Backbone Amide Solvent Accessibility. In contrast to the NMR experiments, the strong interaction between the GpIb $\alpha\left(\mathrm{Y}_{\mathrm{P}}{ }^{276} \mathrm{Y}_{\mathrm{P}}{ }^{278} \mathrm{Y}_{\mathrm{P}}{ }^{279}\right)$ and IIa is an important asset when performing HDX (see Chapter III). Chapter IV describes the sequence coverage obtained from a peptic digest of IIa with or without the aid of the reducing agent TCEP (Figure 25). The fourteen fragments contain coverage for ABE-I, ABE-II, the autolysis loop, the $\mathrm{Na}^{+}$site, the A-chain, and residues involved in substrate recognition and catalysis.

The GpIb $\alpha\left(\mathrm{Y}_{\mathrm{P}}{ }^{276} \mathrm{Y}_{\mathrm{P}}{ }^{278} \mathrm{Y}_{\mathrm{P}}{ }^{279}\right)$ peptide $(1 \mathrm{mM})$ was incubated with IIa $(50 \mu \mathrm{M})$ at room temperature for one or ten minutes in the presence of $99.99 \% \mathrm{D}_{2} \mathrm{O}$. The $\mathrm{K}_{\mathrm{D}}$ for the interaction of the GpIb $\alpha$ (269-286) peptide with IIa has not been determined. A previous study found that GpIbo residues $265-285$ bound to IIa with a $\mathrm{K}_{\mathrm{D}}$ of $5.9 \mathrm{nM}(101)$. In addition, the NMR results suggest that the binding of the GpIb $\alpha$ (269-286) peptide to IIa is probably tighter than $\gamma^{\prime}$ peptide $(410-427)\left(\mathrm{K}_{\mathrm{D}}=680 \mathrm{nM}\right)(69)$. Therefore, similar HDX concentrations of the peptide and IIa (20:1) were used as described in Chapter IV.

The results for deuterium incorporation are compiled in Table 7 and Figure 35. The GpIb $\alpha$ peptide significantly protects fragments spanning residues 85-99 (Figure 36) and 173-181 from deuterium. In fact, the protection from solvent, as represented by negative $\%$ differences in Table 8 , increases from one to ten minutes suggesting this peptide is binding to ABE-II. These results are similar in magnitude to the effects of the $\gamma^{\prime}$ peptide on deuterium exchange at ABE-II (Table 8). Within one minute of deuteration, the GpIb $\alpha$ peptide protects the fragment $85-99$ by 2.7 deuterons $(-17.3 \%)$ and the $\gamma^{\prime}$ peptide by 2.5 deuterons (-16.4\%) (Figure 36B, C, and D). After 10 minutes of deuteration, the 
GpIb $\alpha$ peptide shields the same fragment from solvent by 3.7 deuterons $(-23.9 \%)$ and the $\gamma^{\prime}$ peptide by 3.4 deuterons $(-21.8 \%$ ) (Figure $36 \mathrm{H}$, I, and J).

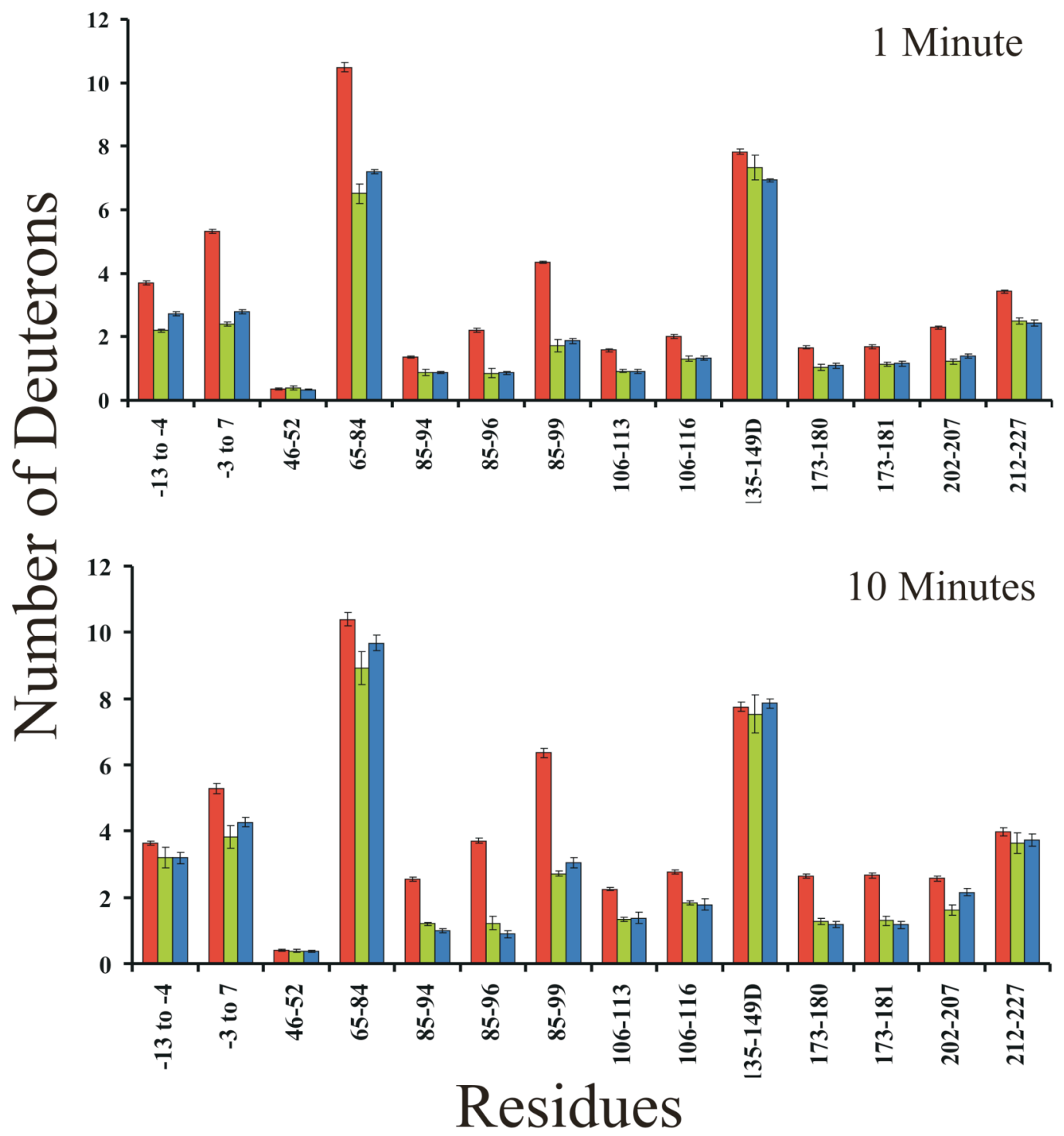

Figure 35: Graphs of deuterium incorporation for IIa, GpIb $\alpha$-IIa, and $\gamma^{\prime}$-IIa at 20:1. The bars in the graph correspond to IIa alone (red), GpIb $\alpha$-IIa (green) and $\gamma^{\prime}$ peptide-IIa (blue). Errors correspond to the standard deviation of the mean for three independent experiments.

As with the $\gamma^{\prime}$ peptide, the GpIb $\alpha$ peptide protects regions within IIa unaffiliated with binding at ABE-II. Most of these peptides are more shielded from deuterium within one minute of exchange compared to ten minutes. These fragments are considered not to be completely solvent excluded in the presence of the GpIb $\alpha$ peptide. The regions 
affected by GpIb $\alpha$ binding to ABE-II after one minute of deuterium exchange include the ABE-I fragments 65-84 (-18.3\%), 106-113 (-9.9\%), and 106-116 (-7.2\%), the A-chain residues -13 to $-4(-17.8 \%)$ and -3 to $7(-17.3 \%)$ and the nearby B-chain fragment 202207 (-15.6\%), and finally the $\mathrm{W}^{215} / \mathrm{Na}^{+}$binding site region $212-227(-5.8 \%)$ (Table 8).

\begin{tabular}{|c|c|c|c|c|c|c|}
\hline \multirow{3}{*}{ Residues } & \multirow{3}{*}{$\begin{array}{l}\text { Theo. } \\
\mathrm{D}_{\max }\end{array}$} & \multicolumn{5}{|c|}{1 Minute } \\
\hline & & \multirow[b]{2}{*}{ IIa } & \multicolumn{2}{|c|}{$20: 1$} & \multicolumn{2}{|c|}{$40: 1$} \\
\hline & & & GpIb $\alpha$ IIa & $\gamma^{\prime}$ IIa & GpIb $\alpha$ IIa & $\gamma^{\prime}$ IIa \\
\hline-13 to $-4^{b}$ & 8.5 & $3.7 \pm 0.1$ & $2.2 \pm 0.1$ & $2.7 \pm 0.1$ & $\mathrm{n} / \mathrm{a}^{c}$ & $2.2 \pm 0.0$ \\
\hline-3 to 7 & 16.9 & $5.3 \pm 0.1$ & $2.4 \pm 0.1$ & $2.8 \pm 0.1$ & $3.3 \pm 0.0$ & $2.1 \pm 0.3$ \\
\hline $46-52$ & 6.6 & $0.4 \pm 0.0$ & $0.4 \pm 0.1$ & $0.3 \pm 0.0$ & $0.4 \pm 0.0$ & $0.3 \pm 0.0$ \\
\hline $65-84$ & 21.8 & $10.5 \pm 0.1$ & $6.5 \pm 0.3$ & $7.2 \pm 0.1$ & $2.6 \pm 0.1$ & $5.6 \pm 0.1$ \\
\hline $85-94$ & 8.8 & $1.4 \pm 0.0$ & $0.9 \pm 0.1$ & $0.9 \pm 0.0$ & $1.1 \pm 0.1$ & $0.8 \pm 0.1$ \\
\hline $85-96$ & 10.9 & $2.2 \pm 0.1$ & $0.9 \pm 0.2$ & $0.9 \pm 0.1$ & $1.3 \pm 0.1$ & $0.7 \pm 0.1$ \\
\hline $85-99$ & 15.2 & $4.4 \pm 0.0$ & $1.7 \pm 0.2$ & $1.9 \pm 0.1$ & $2.1 \pm 0.3$ & $1.6 \pm 0.2$ \\
\hline $106-113^{b}$ & 6.7 & $1.6 \pm 0.0$ & $0.9 \pm 0.0$ & $0.9 \pm 0.1$ & $\mathrm{n} / \mathrm{a}$ & $0.8 \pm 0.1$ \\
\hline $106-116^{b}$ & 9.8 & $2.0 \pm 0.1$ & $1.3 \pm 0.1$ & $1.3 \pm 0.1$ & $\mathrm{n} / \mathrm{a}$ & $1.2 \pm 0.1$ \\
\hline 135-149D & 19.4 & $7.8 \pm 0.1$ & $7.3 \pm 0.4$ & $6.9 \pm 0.0$ & $3.3 \pm 0.1$ & $6.3 \pm 0.0$ \\
\hline $173-180^{b}$ & 7.8 & $1.7 \pm 0.1$ & $1.0 \pm 0.1$ & $1.1 \pm 0.1$ & $\mathrm{n} / \mathrm{a}$ & $1.0 \pm 0.1$ \\
\hline $173-181^{b}$ & 8.8 & $1.7 \pm 0.1$ & $1.1 \pm 0.1$ & $1.2 \pm 0.1$ & $\mathrm{n} / \mathrm{a}$ & $1.1 \pm 0.1$ \\
\hline $202-207^{b}$ & 6.8 & $2.3 \pm 0.0$ & $1.2 \pm 0.1$ & $1.4 \pm 0.1$ & $\mathrm{n} / \mathrm{a}$ & $1.2 \pm 0.1$ \\
\hline $212-227$ & 15.9 & $3.4 \pm 0.1$ & $2.5 \pm 0.1$ & $2.4 \pm 0.1$ & $2.5 \pm 0.1$ & $2.4 \pm 0.1$ \\
\hline \multirow{3}{*}{ Residues } & \multirow{3}{*}{$\begin{array}{l}\text { Theo. } \\
\mathrm{D}_{\max }\end{array}$} & \multicolumn{5}{|c|}{10 Minutes } \\
\hline & & \multirow[b]{2}{*}{ IIa } & \multicolumn{2}{|c|}{$20: 1$} & \multicolumn{2}{|c|}{$40: 1$} \\
\hline & & & GpIb $\alpha$ IIa & $\gamma^{\prime}$ IIa & GpIb $\alpha$ IIa & $\gamma^{\prime}$ IIa \\
\hline-13 to $-4^{b}$ & 8.5 & $3.6 \pm 0.1$ & $3.2 \pm 0.3$ & $3.2 \pm 0.2$ & $\mathrm{n} / \mathrm{a}$ & $3.3 \pm 0.1$ \\
\hline-3 to 7 & 16.9 & $5.3 \pm 0.1$ & $3.8 \pm 0.4$ & $4.3 \pm 0.1$ & $6.6 \pm 0.1$ & $4.0 \pm 0.1$ \\
\hline $46-52$ & 6.6 & $0.4 \pm 0.0$ & $0.4 \pm 0.0$ & $0.4 \pm 0.0$ & $0.6 \pm 0.0$ & $0.4 \pm 0.0$ \\
\hline $65-84$ & 21.8 & $10.4 \pm 0.2$ & $8.9 \pm 0.5$ & $9.7 \pm 0.2$ & $7.6 \pm 0.1$ & $9.6 \pm 0.3$ \\
\hline $85-94$ & 8.8 & $2.6 \pm 0.1$ & $1.2 \pm 0.1$ & $1.0 \pm 0.1$ & $3.1 \pm 0.1$ & $1.1 \pm 0.1$ \\
\hline $85-96$ & 10.9 & $3.7 \pm 0.1$ & $1.2 \pm 0.2$ & $0.9 \pm 0.1$ & $4.0 \pm 0.1$ & $1.2 \pm 0.1$ \\
\hline $85-99$ & 15.2 & $6.4 \pm 0.1$ & $2.7 \pm 0.1$ & $3.0 \pm 0.2$ & $6.2 \pm 0.1$ & $2.5 \pm 0.1$ \\
\hline $106-113^{b}$ & 6.7 & $2.3 \pm 0.0$ & $1.3 \pm 0.1$ & $1.4 \pm 0.2$ & $\mathrm{n} / \mathrm{a}$ & $1.4 \pm 0.0$ \\
\hline $106-116^{b}$ & 9.8 & $2.8 \pm 0.1$ & $1.8 \pm 0.1$ & $1.8 \pm 0.2$ & $\mathrm{n} / \mathrm{a}$ & $1.8 \pm 0.1$ \\
\hline 135-149D & 19.4 & $7.7 \pm 0.1$ & $7.5 \pm 0.6$ & $7.8 \pm 0.1$ & $8.2 \pm 0.0$ & $8.0 \pm 0.1$ \\
\hline $173-180^{b}$ & 7.8 & $2.6 \pm 0.1$ & $1.3 \pm 0.1$ & $1.2 \pm 0.1$ & $\mathrm{n} / \mathrm{a}$ & $1.3 \pm 0.1$ \\
\hline $173-181^{b}$ & 8.8 & $2.7 \pm 0.1$ & $1.3 \pm 0.1$ & $1.2 \pm 0.1$ & $\mathrm{n} / \mathrm{a}$ & $1.2 \pm 0.1$ \\
\hline $202-207^{b}$ & 6.8 & $2.6 \pm 0.1$ & $1.6 \pm 0.2$ & $2.2 \pm 0.1$ & $\mathrm{n} / \mathrm{a}$ & $1.7 \pm 0.1$ \\
\hline $212-227$ & 15.9 & $4.0 \pm 0.1$ & $3.6 \pm 0.3$ & $3.7 \pm 0.2$ & $5.4 \pm 0.0$ & $3.9 \pm 0.2$ \\
\hline
\end{tabular}

${ }^{a}$ The maximum number of exchangeable protons within the indicated peptide, assuming $100 \%$ deuteration (Chapter III). A fully deuterated peptide would theoretically have acquired this amount of deuterons. ${ }^{b}$ The deuterium uptake for these peptides was quantified from the nonreducing digests. All other peptides are from the TCEP digests. When isotopic clusters appear in both the TCEP and non-TCEP digests, the differences in deuteration are negligible when comparing similar peptides. Certain peptides appear exclusively under non-reducing or reducing conditions, hence the necessity of performing both types of peptic digests. ${ }^{c}$ These fragments were not able to be quantified in the non-reducing digest at 40:1 GpIb $\alpha$ to IIa. 


\section{Residues 85-99}

1 Minute
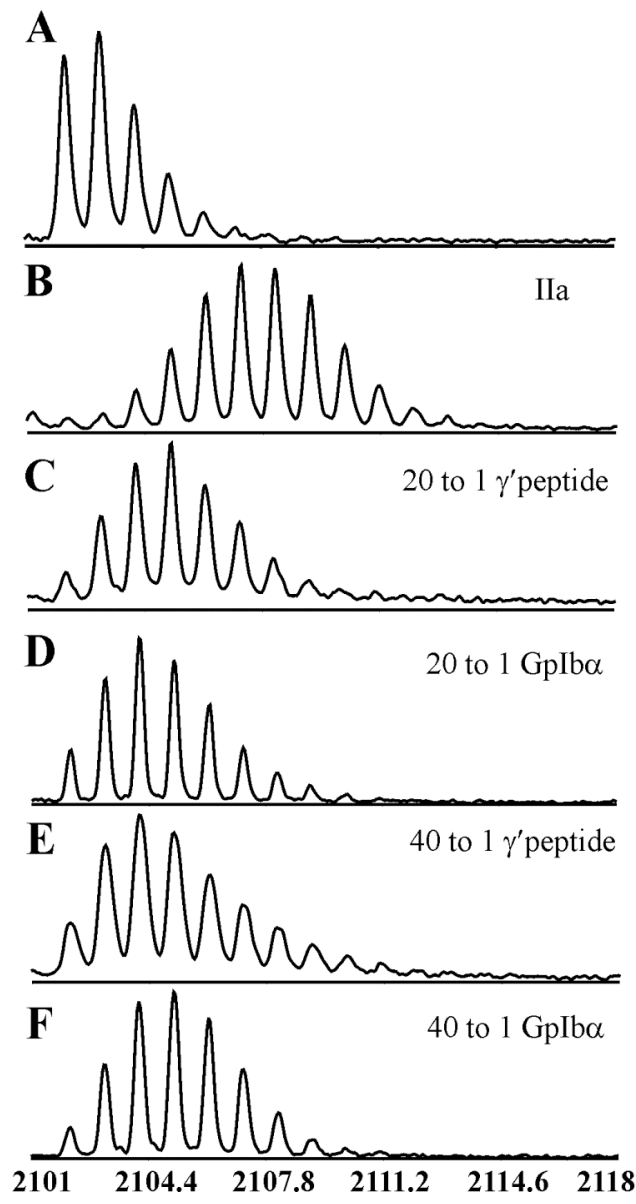

10 Minutes
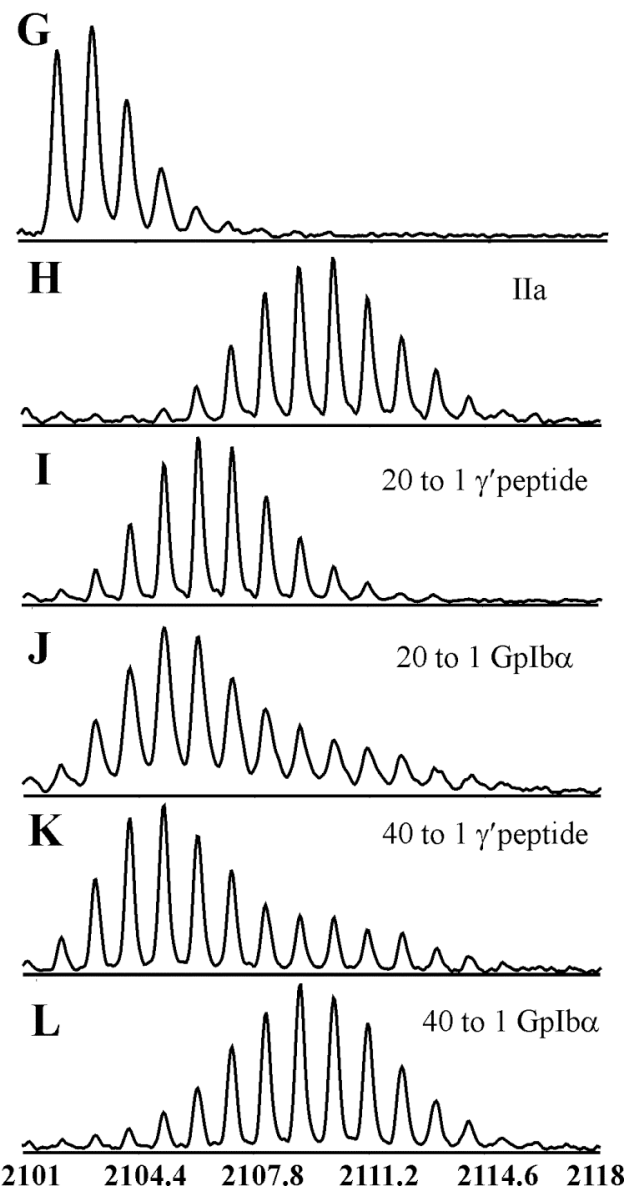

Figure 36: Mass spectra of residues 85-99 after one and ten minutes of HDX. All of the D-on experiments are in $150 \mathrm{mM} \mathrm{NaCl}, 25 \mathrm{mM} \mathrm{NaH}_{2} \mathrm{PO}_{3}, \mathrm{pH}$ 6.5. A and G: the undeuterated peak cluster, B and H: IIa spectra in the absence of ligands, C and I: $\gamma^{\prime}$ peptide $(1 \mathrm{mM})$ bound to IIa $(50 \mu \mathrm{M})$ at a 20:1 ratio, D and $\mathrm{J}$ : GpIbo peptide $(1 \mathrm{mM})$ bound to IIa $(50 \mu \mathrm{M})$ at a $20: 1$ ratio, E and $\mathrm{K}: \gamma^{\prime}$ peptide $(2 \mathrm{mM})$ bound to IIa $(50 \mu \mathrm{M})$ at a 40:1 ratio, and F and L: GpIbo peptide $(2 \mathrm{mM})$ bound to $\mathrm{IIa}(50 \mu \mathrm{M})$ at a $40: 1$ ratio. At the ratio of $20: 1$, both peptides exhibit a similar degree of protection for these ABE-II residues; however in spectrum $\mathrm{J}$ a small shoulder is present suggesting the presence of two IIa populations. Increasing the amount of peptide completely abrogates protection after ten minutes in the presence of the GpIb $\alpha$ peptide, while the $\gamma^{\prime}$ peptide begins to display a similar shoulder at ten minutes.

After ten minutes of deuteration, the two A-chain fragments lose some degree of protection from solvent, with -13 to -4 down to $-5.2 \%$ and -3 to 7 reduced to $-8.6 \%$; however, the nearby B-chain fragment $202-207$ is at a similar level compared to one 
minute $(-14.1 \%)$. The ABE-I fragment $65-84(-6.7 \%)$ also is reduced in the observed amount of deuterium protection. The segments 106-113 (-13.7\%) and 106-116 (-9.5\%) experience slight increases in protection at ten minutes. The $\mathrm{W}^{215} / \mathrm{Na}^{+}$binding site peptide $212-227(-2.1 \%)$ ceases to be significantly protected from solvent after ten minutes of deuteration. GpIb $\alpha$ binding does not change the dynamics of deuterium exchange for the fragments 46-52 and 135-149D (the autolysis loop).

\begin{tabular}{|c|c|c|c|c|c|c|c|c|c|}
\hline \multirow{3}{*}{ Residues } & \multirow{3}{*}{$\begin{array}{l}\text { Theo } \\
\mathrm{D}_{\max }\end{array}$} & \multicolumn{4}{|c|}{$20: 1$} & \multicolumn{4}{|c|}{$40: 1$} \\
\hline & & \multicolumn{2}{|c|}{ GpIb $\alpha$ IIa } & \multicolumn{2}{|c|}{$\gamma^{\prime}$ IIa } & \multicolumn{2}{|c|}{ GpIb $\alpha$ IIa } & \multicolumn{2}{|c|}{$\gamma^{\prime}$ IIa } \\
\hline & & $1 \mathrm{~min}$ & $10 \mathrm{~min}$ & $1 \mathrm{~min}$ & $10 \mathrm{~min}$ & $1 \mathrm{~min}$ & $10 \mathrm{~min}$ & $1 \mathrm{~min}$ & $10 \mathrm{~min}$ \\
\hline-13 to $-4^{c}$ & 8.5 & $-17.8^{\alpha}$ & -5.2 & -11.3 & -5.1 & $\mathrm{n} / \mathrm{a}$ & $\mathrm{n} / \mathrm{a}$ & -17.3 & -4.0 \\
\hline-3 to 7 & 16.9 & -17.3 & -8.6 & -15.0 & -6.1 & -12.0 & 7.8 & -19.1 & -7.9 \\
\hline $46-52$ & 6.6 & 0.5 & -0.5 & -0.2 & -0.7 & 0.8 & 3.0 & -0.3 & -0.2 \\
\hline $65-84$ & 21.8 & -18.3 & -6.7 & -15.1 & -3.3 & -36.2 & -12.7 & -22.3 & -3.8 \\
\hline $85-94$ & 8.8 & -5.5 & -15.3 & -5.6 & -17.7 & -3.4 & 6.3 & -6.3 & -16.9 \\
\hline $85-96$ & 10.9 & -12.4 & -22.8 & -12.3 & -25.9 & -8.2 & 2.8 & -13.7 & -22.8 \\
\hline $85-99$ & 15.2 & -17.3 & -23.9 & -16.4 & -21.8 & -14.8 & -1.1 & -18.0 & -25.7 \\
\hline $106-113^{c}$ & 6.7 & -9.9 & -13.7 & -10.0 & -13.1 & $\mathrm{n} / \mathrm{a}$ & $\mathrm{n} / \mathrm{a}$ & -11.0 & -12.8 \\
\hline $106-116^{c}$ & 9.8 & -7.2 & -9.5 & -6.9 & -10.0 & $\mathrm{n} / \mathrm{a}$ & $\mathrm{n} / \mathrm{a}$ & -8.1 & -9.5 \\
\hline $135-149 \mathrm{D}$ & 19.4 & -2.5 & -1.1 & -4.6 & -0.5 & -23.2 & 2.3 & -7.8 & 1.0 \\
\hline $173-180^{c}$ & 7.8 & -8.1 & -17.6 & -7.3 & -18.6 & $\mathrm{n} / \mathrm{a}$ & $\mathrm{n} / \mathrm{a}$ & -8.5 & -17.6 \\
\hline $173-181^{c}$ & 8.8 & -6.3 & -15.5 & -5.9 & -16.9 & $\mathrm{n} / \mathrm{a}$ & $\mathrm{n} / \mathrm{a}$ & -6.4 & -16.5 \\
\hline $202-207^{c}$ & 6.8 & -15.6 & -14.1 & -13.2 & -6.1 & $\mathrm{n} / \mathrm{a}$ & $\mathrm{n} / \mathrm{a}$ & -15.7 & -12.2 \\
\hline $212-227$ & 15.9 & -5.8 & -2.1 & -6.3 & -1.5 & -6.0 & 9.2 & -6.5 & -0.7 \\
\hline
\end{tabular}

${ }^{a}$ The $\%$ change for a particular peptide is calculated by Equation 7 (Chapter III). ${ }^{b}$ The maximum number of exchangeable protons within the indicated peptide, assuming $100 \%$ deuteration. (Chapter III). A fully deuterated peptide would theoretically have acquired this amount of deuterons. ${ }^{c}$ The deuterium uptake for these peptides was quantified from the non-reducing digests. All other peptides are from the TCEP digests. When isotopic clusters appear in both the TCEP and non-TCEP digests, the differences in deuteration are negligible when comparing similar peptides. Certain peptides appear exclusively under non-reducing or reducing conditions, hence the necessity of performing both types of peptic digests. ${ }^{d}$ The values in bold represent significant changes in deuteration of greater than $-4.5 \%$. ${ }^{e}$ These fragments were not able to be quantified in the non-reducing digest at 40:1 GpIb $\alpha$ to IIa.

When comparing the amount of deuterium incorporation for these fragments in the presence of the GpIb $\alpha$ peptide to the $\gamma^{\prime}$ peptide, very similar trends are observed for the segments -13 to $-4,-3$ to $7,106-113,106-116$, and 212-227. Differences do exist for two peptides in regard to the amount of protection still present after ten minutes. The ABE-I residues $65-84$ (-6.7\% for GpIba vs. $-3.3 \%$ for $\left.\gamma^{\prime}\right)$ and the peptide $202-207(-14.1 \%$ for 
GpIb $\alpha$ vs. $-6.1 \%$ for $\gamma^{\prime}$ ) are more protected at ten minutes by the GpIb $\alpha$ peptide than the $\gamma^{\prime}$ peptide (Table 8). In addition, the $\gamma^{\prime}$ peptide exhibits protection from solvent for the autolysis loop fragment 135-149D (-4.6\%) after one minute, whereas the GpIb $\alpha$ peptide does not appear to influence this region. These results suggest that GpIba binding to IIa has subtly different consequences on protein dynamics when compared to the $\gamma^{\prime}$ peptide.

In Figure 36J, the isotopic cluster for residues 85-99 has an interesting feature not observed with the other fragments in the peptic digest. The cluster appears to be expanded with a slight shoulder ending near $2115 \mathrm{~m} / z$. This larger peak envelope was observed consistently in each experiment when the concentration of GpIb $\alpha$ was $1 \mathrm{mM}$ and IIa was $50 \mu \mathrm{M}$ (20:1 molar ratio). The results suggest the presence of two different IIa populations exposed to deuterium during HDX. In order to test this theory, HDX was performed on a higher ratio (40:1) of peptide $(2 \mathrm{mM})$ to IIa $(50 \mu \mathrm{M})$. Perhaps the newly emerging second population of IIa would eventually dominate the results.

HDX Experiments (II): Examining the Influence of a Higher Peptide Concentration on the Dynamics of Thrombin's Deuterium Incorporation. In HDX experiments with 40:1 GpIb $\alpha$ peptide to IIa, only the digests with TCEP presented reliable isotopic clusters for quantification. Thus, the amount of deuteration for the fragments -13 to $-4,106-113$, 106-116, 173-180, 173-181, and 202-207 was unable to be reported at this GpIbo peptide concentration $(2 \mathrm{mM})$. These problems were not encountered with the $\gamma^{\prime}$ peptide and the data for all fourteen peptides are presented in Tables 7 and 8 . Figure 37 graphically illustrates the data for HDX at 40:1 peptide to thrombin.

For the $\gamma^{\prime}$ peptide, deuterium incorporation for the fragments representing ABE-II residues 85-99 (Figures 36E and $\mathrm{K}$ ) and 173-181 were similar at $2 \mathrm{mM}$ peptide (40:1) as 


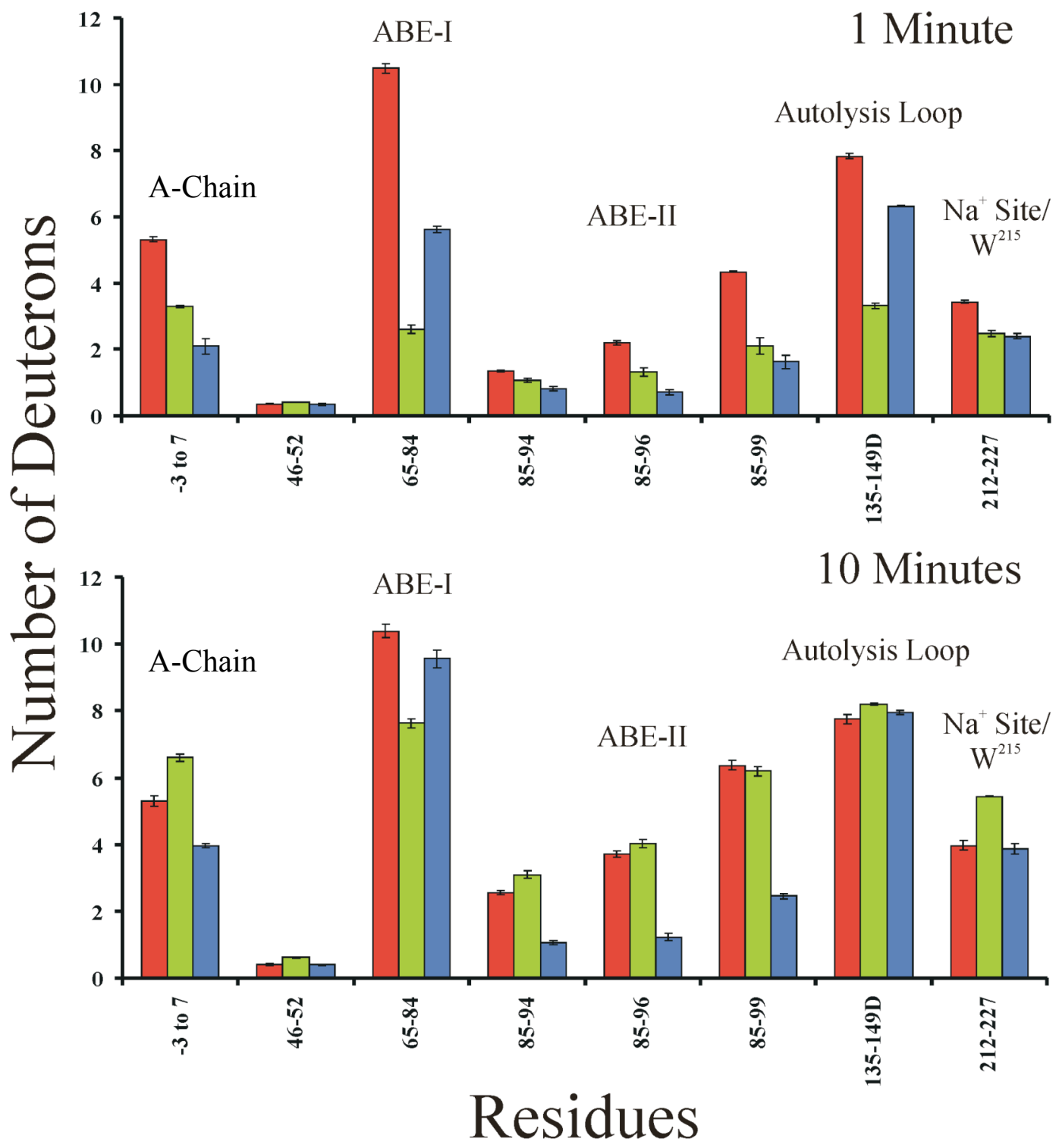

Figure 37: Graphs of deuterium incorporation for IIa, GpIb $\alpha$-IIa, and $\gamma^{\prime}$-IIa at 40:1. These results are taken from the digests with the reducing agent TCEP. The bars in the graph correspond to IIa alone (red), GpIb $\alpha$-IIa (green) and $\gamma^{\prime}$ peptide-IIa (blue). Errors correspond to the standard deviation of the mean for three independent experiments.

at $1 \mathrm{mM}$ peptide (20:1) (Table 8 ). Interestingly, Figure 36K displays the appearance of a similar shoulder on the 85-99 isotopic cluster for 40:1 $\gamma^{\prime}$ peptide-IIa that was observed with the GpIbo peptide during the 20:1 experiments (Figure 36I). In fact, many of the other fragments obtained from the non-reducing digests are analogous to the results for GpIb $\alpha$ at 20:1. The A-chain residues -13 to -4 are initially protected to a greater degree 
(-17.3\% for $40: 1$ vs. $-11.3 \%$ for $20: 1)$ and residues $202-207$ maintain a steady difference in deuterium incorporation over ten minutes $(-15.7 \%$ at one minute to $-12.2 \%$ at ten minutes) (Table 8). The ABE-I fragment 106-116 is shielded from deuterium in a similar manner at both peptide concentrations. The only significant difference between 40:1 $\gamma^{\prime}$ peptide and 20:1 GpIb $\alpha$ peptide is apparent in the autolysis loop, where the $\gamma^{\prime}$ peptide protects residues 135-149D from deuterium after 1 minute of exchange.

GpIb $\alpha$ binding to IIa at a higher peptide concentration reveals a different mechanism for peptide interaction. Figure 38 illustrates the one minute HDX spectra for ABE-I residues 65-84. At 40:1 and one minute of HDX, the GpIbo peptide imparts a dramatic amount of protection for $65-84$ amounting to 7.9 deuterons up from 4.0 deuterons at 20:1 (Figure 38D and F; Tables 7). Even after ten minutes of deuteration, the protection is 2.8 deuterons compared to 1.5 deuterons at $20: 1$. The $\gamma^{\prime}$ peptide at both HDX concentrations shows a significant amount of initial protection (Figure 38C and E), which dissipates after ten minutes of deuteration. Examining the effect of GpIba on ABE-II, residues 85-94 are not shielded from HDX after one minute (-3.4\%); however, within ten minutes, these residues are more exposed to solvent (+6.3\%). HDX in 85-96 and 85-99 reveal protection from solvent within a minute of deuterium exposure, but ten minutes of HDX leaves these residues at similar levels to IIa alone (Figure 36H and L).

Three additional IIa regions display new responses to GpIb $\alpha$ peptide binding. Both the A-chain fragment -3 to 7 and the $\mathrm{W}^{215} / \mathrm{Na}^{+}$binding site peptide (212-227) are shielded from deuterium within one minute $(-12.0 \%$ and $-6.0 \%$ respectively); however ten minutes of deuterium exposure results in both of these regions becoming more available for deuterium exchange when compared to IIa alone $(+7.8 \%$ and $+9.2 \%$ 
respectively) (Table 8). With the autolysis loop, 20:1 GpIbo peptide did not affect the amount of deuterium incorporation relative to free IIa. By increasing the concentration of the GpIb $\alpha$ peptide (40:1), the residues $135-149 \mathrm{D}$ are now protected by $-23.2 \%$ within a minute of deuterium exchange and that protection is abrogated after ten minutes $(+2.3 \%)$.

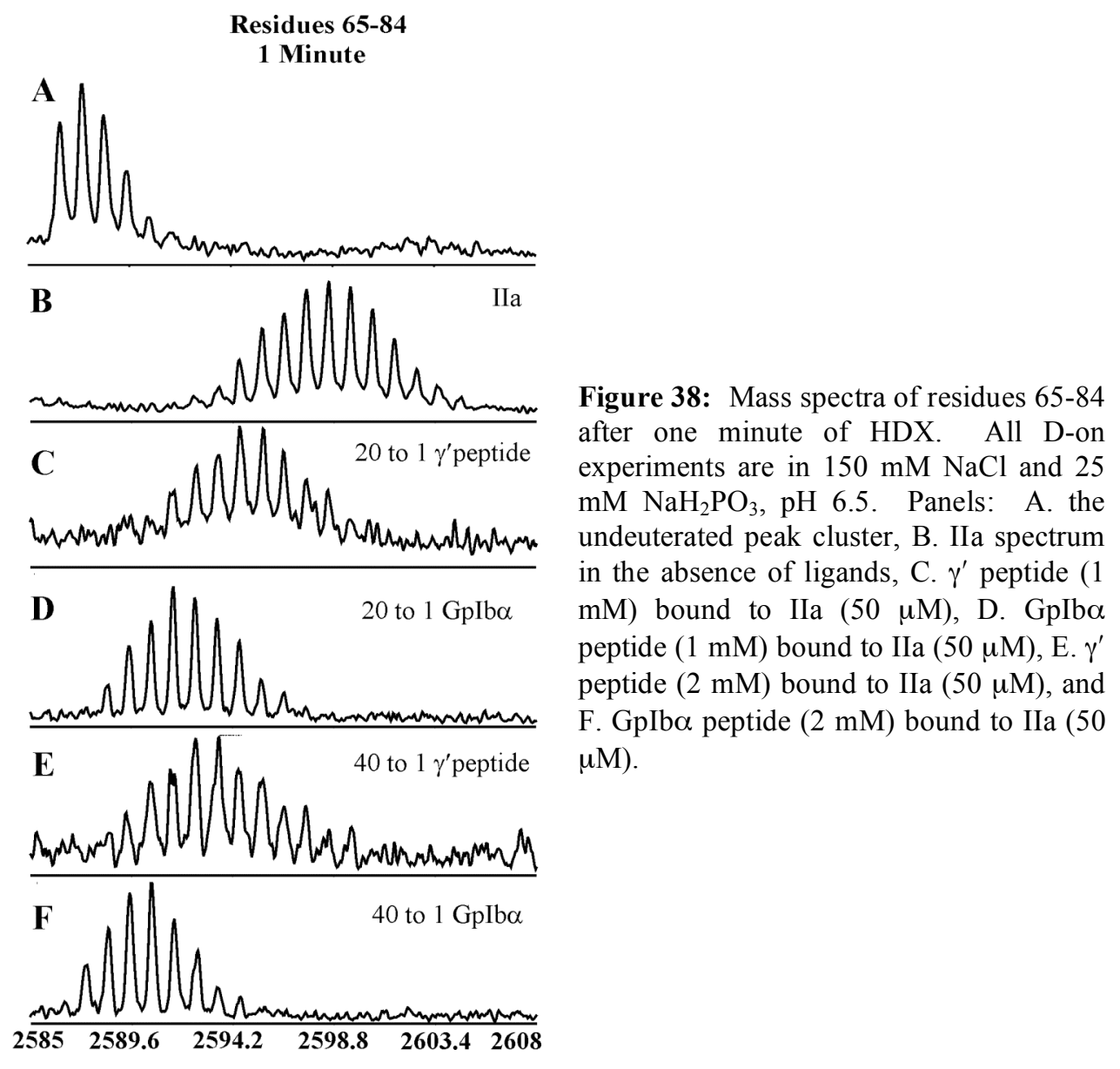

Analytical Ultracentrifugation. AUC was employed to determine whether the GpIb $\alpha$ peptide or the $\gamma^{\prime}$ peptide induces dimerization of IIa in solution. In these AUC trials, the concentrations of PPACK inhibited IIa spanned 4.7-7.0 $\mu \mathrm{M}$. A series of sedimentation velocity experiments were executed with a range of peptide to IIa molar ratios varying from 1:1 to 20:1. Two trials with IIa alone were performed for comparison to the peptide-IIa experiments. Table 9 presents the data for the average of two runs. 
Figures 39 and 40 illustrate the raw SV data used to construct the distribution of Svedberg constants (S) for the trial examining the GpIbo peptide and IIa at a 2:1 ratio and the $\gamma^{\prime}$ peptide and IIa at $1: 1$, respectively.

The Svedberg constant measures the rate of sedimentation, which is related to the size and shape of the molecule. It should be noted that this constant is not additive, though molecular weight is additive. In other words, $\mathrm{S}$ values are affected by the amount of surface area exposed to solvent. Thus when two proteins interact, the surface area is decreased leading to a non-additive increase in the S constant. The calculated Svedberg constants for the peptide binding to the enzyme range from 3.2-3.3, compared to 3.1 for IIa alone. The slight increase in molecular weight with increasing peptide concentration coupled with the slight increase in $\mathrm{S}$ values seem to propose that the peptides bind to IIa but appear not to instigate protein dimerization.

\begin{tabular}{|c|c|c|}
\hline & Sedimentation Coefficient (S) & Molecular Weight $(\mathrm{kDa})$ \\
\hline IIa alone & $3.11 \pm 0.00$ & $36.2 \pm 0.3$ \\
\hline \multicolumn{3}{|c|}{ GpIb $\alpha$ peptide:IIa } \\
\hline $1: 1$ & $3.19 \pm 0.02$ & $37.3 \pm 0.0$ \\
\hline $2: 1$ & $3.18 \pm 0.01$ & $37.5 \pm 0.9$ \\
\hline $5: 1$ & $3.27 \pm 0.05$ & $37.9 \pm 0.7$ \\
\hline $10: 1$ & $3.25 \pm 0.02$ & $37.2 \pm 1.1$ \\
\hline $20: 1$ & $3.22 \pm 0.01$ & $38.1 \pm 1.4$ \\
\hline \multicolumn{3}{|c|}{$\gamma^{\prime}$-peptide:IIa } \\
\hline $1: 1$ & $3.19 \pm 0.02$ & $39.3 \pm 1.0$ \\
\hline $2: 1$ & $3.22 \pm 0.03$ & $38.6 \pm 0.4$ \\
\hline $5: 1$ & $3.23 \pm 0.03$ & $39.9 \pm 0.2$ \\
\hline $10: 1$ & $3.27 \pm 0.01$ & $39.9 \pm 0.6$ \\
\hline $20: 1$ & $3.28 \pm 0.01$ & $40.9 \pm 0.7$ \\
\hline
\end{tabular}



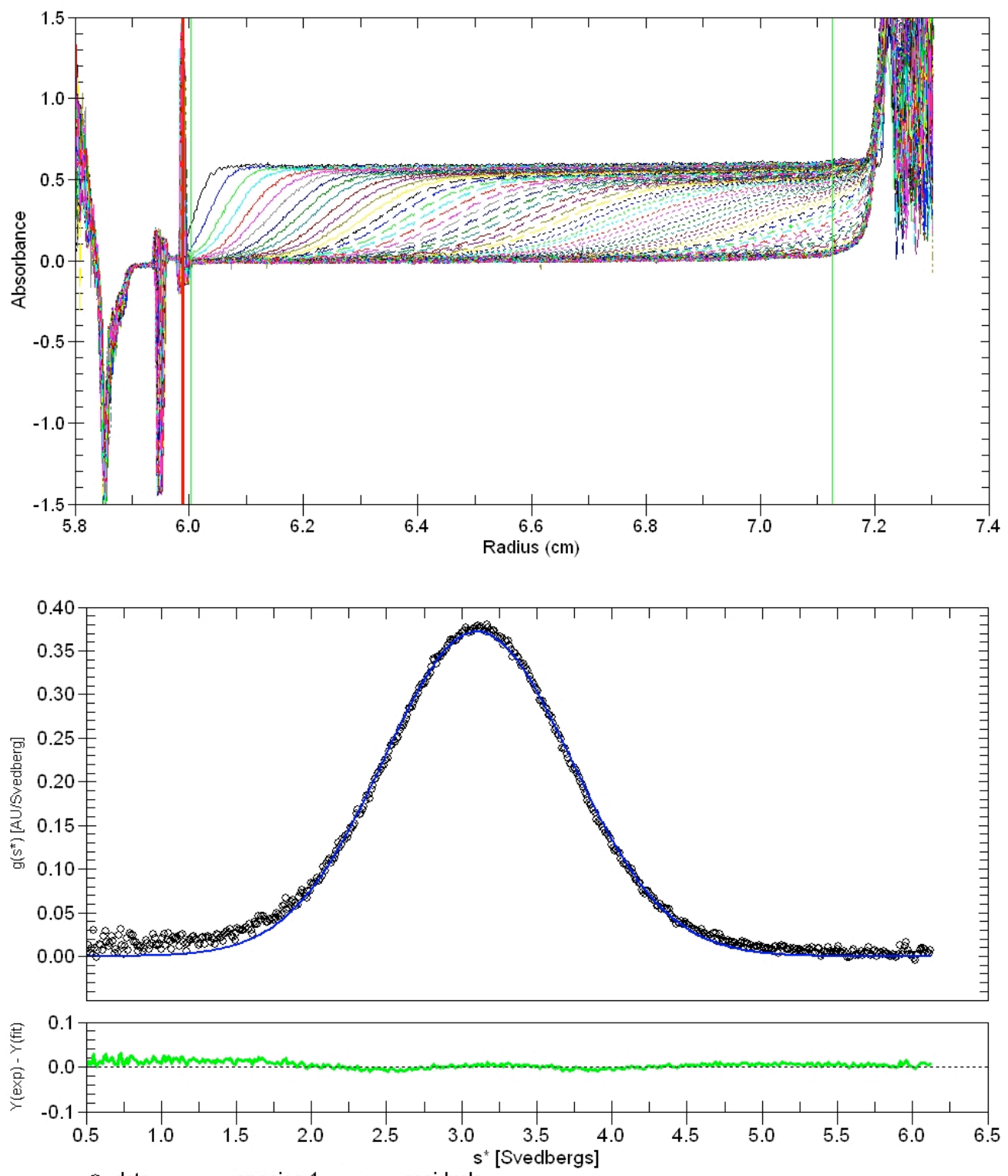

0 data species 1 residuals

Figure 39: Sedimentation velocity AUC of GpIb $\alpha$ peptide $(14 \mu \mathrm{M})$ and IIa $(7 \mu \mathrm{M})$. Analysis was performed at $280 \mathrm{~nm}, 20^{\circ} \mathrm{C}$, and $60,000 \mathrm{rpm}$ in $150 \mathrm{mM} \mathrm{NaCl}, 25 \mathrm{mM}$ phosphate, $\mathrm{pH}$ 7.4. Top Panel: Raw data set for the sedimentation velocity run examining this interaction. Bottom Panel: The apparent Svedberg constant distribution centered at 3.2 $\mathrm{S}$ with the residuals resulting from a global fit of the data using the DCDT+ program. 

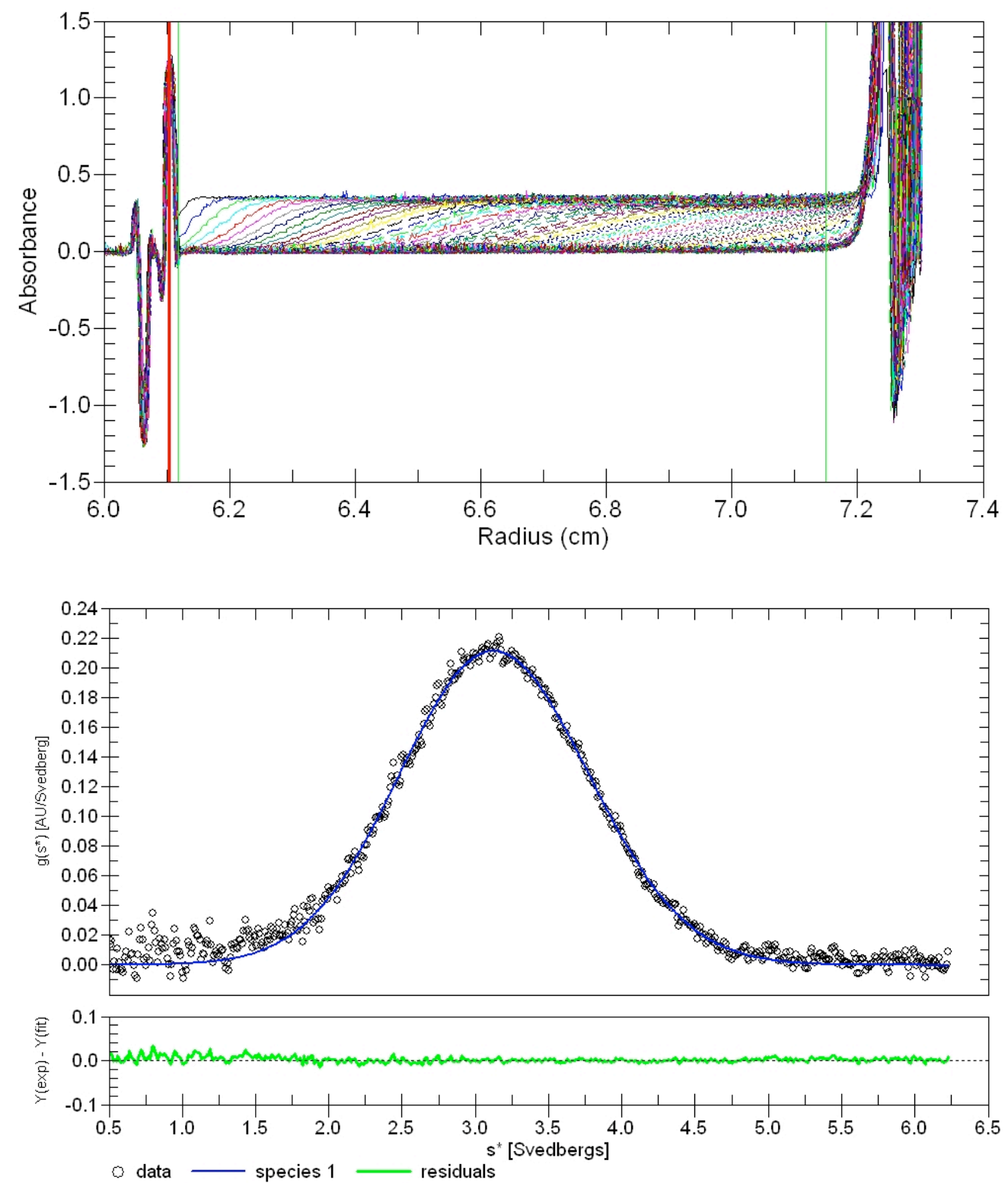

Figure 40: Sedimentation velocity AUC of $\gamma^{\prime}$ peptide $(4.7 \mu \mathrm{M})$ and IIa $(4.7 \mu \mathrm{M})$. Analysis was performed at $280 \mathrm{~nm}, 20^{\circ} \mathrm{C}$ and $60,000 \mathrm{rpm}$ in $150 \mathrm{mM} \mathrm{NaCl}, 25 \mathrm{mM}$ phosphate, $\mathrm{pH}$ 7.4. Top Panel: Raw data set for the sedimentation velocity run examining this interaction. Bottom Panel: The apparent Svedberg constant distribution centered at $3.2 \mathrm{~S}$ with the residuals resulting from a global fit of the data using the DCDT+ program. 


\section{Discussion}

GpIb $\alpha$ binds to IIa, however, the literature presents evidence for interactions at both anion binding exosites. Though mutagenesis data strongly suggest that GpIb $\alpha$ binds exclusively at ABE-II $(23,68,92)$, binding studies display interactions at both exosites $(68,93,95,98,101)$. Adding to the ambiguity, two crystal structures of GpIba-IIa illustrate similar interactions at ABE-II for one IIa molecule, while a symmetry related IIa binds to GpIb $\alpha$ through ABE-I at different GpIb $\alpha$ interfaces $(96,97)$ (Figure 32). The studies described in this chapter use solution NMR, HDX coupled with MALDITOF MS, and AUC to investigate the interaction of the GpIb $\alpha$ peptide with thrombin. The results will be described in terms of the crystal structures when applicable $(96,97)$.

Analysis of NMR Data: the GpIba Peptide Binds to Thrombin in an Extended Conformation. Initially, NMR experiments were performed with the triply phosphorylated GpIb $\alpha$ peptide. The 1D spectra did not display line-broadening and the 2D trNOESY lacked the presence of inter-residue NOEs. These results indicate that the $\mathrm{k}_{\text {off }}$ for this peptide is too slow for these types of NMR experiments (see Chapter II). In order to sufficiently weaken the interaction and observe the trNOESY effect, the GpIb $\alpha$ peptide was dephosphorylated, first at $\mathrm{Y}^{278}$, then at all three $\mathrm{Y}$ residues. Conflicting accounts exist regarding the interaction of unphosphorylated variants of GpIb $\alpha$ and IIa. One report describes unphosphorylated GpIb $\alpha$ as unable to bind to thrombin (252), while another group demonstrated competitive inhibition of FVIII hydrolysis by IIa in the presence of unphosphorylated GpIba peptide (residues 268-282) (65). The present investigation supports the observation that unphosphorylated GpIba can effectively interact with IIa. 
The NMR results from both GpIb $\alpha\left(\mathrm{Y}_{\mathrm{P}}{ }^{276} \mathrm{Y}_{\mathrm{P}}{ }^{279}\right)$ and the unphosphorylated GpIb $\alpha$ peptide can be used to describe the two different conformations of the GpIb $\alpha$ residues 269-277 in both X-ray structures (Figure 41A) $(96,97)$. The residues $\mathrm{D}^{269}-\mathrm{T}^{273}$ of the GpIba peptide do not display line broadening suggesting that these residues do not contribute to IIa binding. Also, the 2D trNOESY spectra do not support the existence of a turn conformation present in the Dumas structure (1P8V) between residues $\mathrm{D}^{274}-\mathrm{D}^{277}$ (96). Thus, the N-terminus of this peptide probably exists as a flexible portion of the GpIb $\alpha$ protein, able to adopt both of the conformations observed in the two structures.

Curiously, these results are at odds with a prior study focusing on the inhibition of IIa binding to platelets and glycocalicin by GpIba based peptides (101). The group showed that residues $\mathrm{E}^{270}-\mathrm{T}^{273}$ are important for inhibiting platelet and glycocalicin interactions with IIa (101). Yet, both X-ray structures show that $\mathrm{D}^{269}-\mathrm{T}^{273}$ does not make electrostatic contacts with residues from thrombin $(96,97)$. Another study demonstrated that residue $\mathrm{D}^{269}-\mathrm{G}^{271}$ are not essential for GpIb $\alpha$ binding $(98)$. The present data and the X-ray structures $(96,97)$ seem to deemphasize $\mathrm{D}^{269}-\mathrm{T}^{273}$ as important for binding IIa.

The 2D trNOESY data in conjunction with the 1D NMR appear to support the Celikel structure (1OOK) more so than the Dumas structure (1P8V). The Celikel X-ray crystal (1OOK) displays the residues 269-284 in an extended conformation sandwiched between two thrombin monomers (Figure 41B) with a trans conformation for $\mathrm{Y}_{\mathrm{P}}{ }^{279}-\mathrm{P}^{280}$ (Figure 41A) (97). Electrostatic interactions are observed for residues $\mathrm{D}^{274}-\mathrm{T}^{284}$ with both ABE-I and ABE-II (Figure 42) (97). Presumably, residues $\mathrm{P}^{280}-\mathrm{G}^{286}$ are too disordered in the Dumas structure (1P8V) to be effectively modeled (96); yet the NMR data displays significant interactions with thrombin for several of the residues within this 
sequence. Assuming the Celikel structure (1OOK) is an accurate representation of a potential mode of GpIb $\alpha$-IIa binding (97), the 1D line broadening support the possibility of GpIba binding to both exosites of thrombin in an extended conformation through dimerization of the protease.

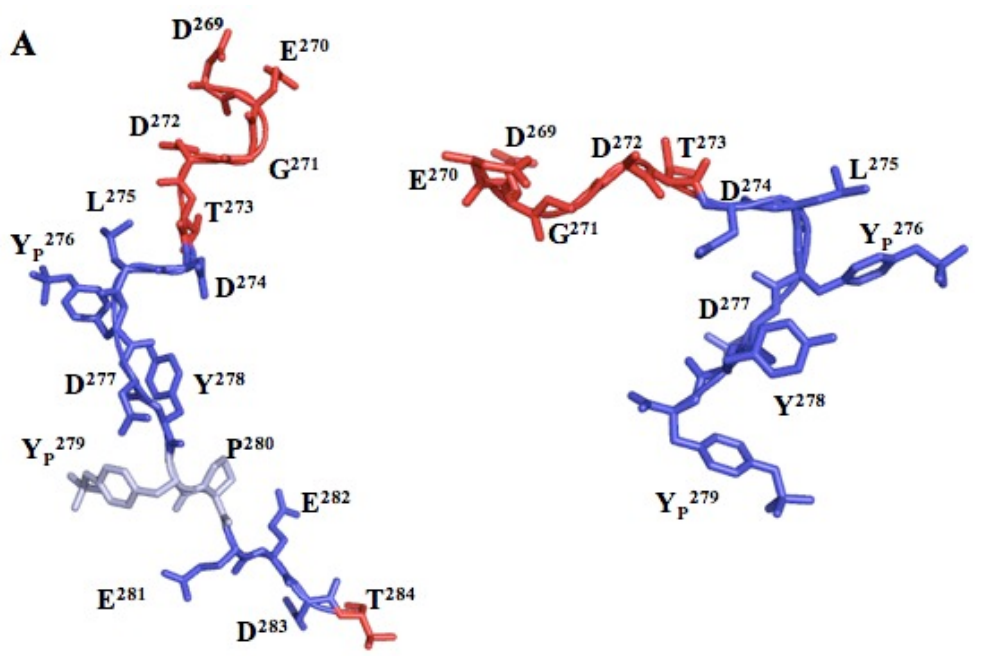

B

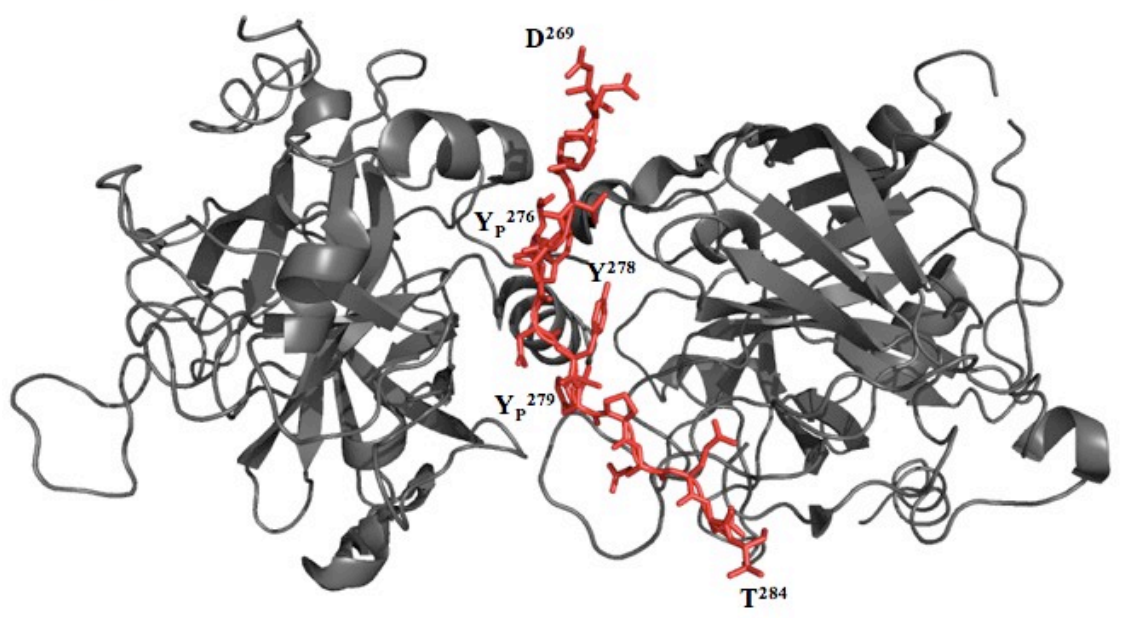

Figure 41: Crystallographic views of GpIb $\alpha$ bound to thrombin (I). A. Representation of the GpIbo peptide isolated from thrombin. The peptide on the left is from 1OOK and the structure on the right is from $1 \mathrm{P} 8 \mathrm{~V}$. The individual amino acids are labeled. The trans bond between $Y_{P}{ }^{279}-P^{280}$ in $100 K$ is in light blue. Residues experiencing line-broadening in the 1D NMR spectra are in blue. B. Illustration of GpIbo peptide induced dimerization of thrombin in 1OOK. The data suggests this structure more accurately represents the NMR results. The C-terminus of GpIba is in an extended conformation and residues $\mathrm{D}^{274}-\mathrm{T}^{284}$ interact with both ABE-II and ABE-I of opposing thrombin monomers. 
Analytical Ultracentrifugation: $\gamma^{\prime}$ Peptide and GpIb $\alpha$ Peptide is Unable to Induce Dimerization of Thrombin. To explain the NMR observations (167), dimerization of IIa appears to be a necessary requirement. (Figures 29B in Chapter IV and Figure 41B) (97, 242). These observations led to AUC being performed with the two peptides and PPACK-IIa (PPACK is an active site inhibitor). It should be noted that the HDX experiments demonstrate that the $\gamma^{\prime}$ peptide interacts effectively with PPACK-IIa (Table 5) (167) and that the GpIb $\alpha$ X-ray structures were solved with PPACK-IIa (96, 97). The AUC results do not depict dimerization of PPACK-IIa by either peptide. The $\mathrm{S}$ values calculated for PPACK inhibited bovine IIa (3.1) and PPACK-II in the presence of the peptides (3.2-3.3) match those previously determined for the exact same form of IIa (3.3) in a similar buffer (253). The same study shows a fragment of thrombomodulin with a similar molecular weight to IIa binding to the serine protease and increasing S from 3.3 to 4.2 (253). Thus, IIa dimerization would lead to an increase in the $\mathrm{S}$ value.

An important discrepancy exists between the AUC studies and the NMR/X-ray investigations. For the NMR/X-ray work, higher concentrations of thrombin are required $(100-150 \mu \mathrm{M})(96,97,167,242)$ whereas in the AUC experiments the concentration of IIa was much lower in order to avoid saturation of the uv-vis detector. For example, 4.7 $\mu \mathrm{M}$ IIa leads to an optical density of 0.41 . Thrombin dimerization by these peptides may also have a IIa concentration dependence. In the future directions section of chapter VII, additional experiments are detailed that could independently confirm IIa dimerization by the peptides, including the measurement of diffusion coefficients by NMR.

Analysis of HDX Results (I): GpIba (269-286) Behaves in a Similar Manner to the $\gamma^{\prime}$ peptide (410-427) when Binding to Thrombin. At a ratio of 20:1 GpIba peptide to IIa, 
the HDX data illustrate very comparable effects to the $\gamma^{\prime}$ peptide (167) on IIa deuterium exchange. The GpIb $\alpha$ peptide appears to bind to IIa's ABE-II based on the deuterium protection for fragments 85-99 and 173-181 over the course of ten minutes (Figure 42). Several residues within these two fragments have been demonstrated to be involved in the interaction by mutagenesis and the X-ray crystallography $(68,92,96,97)$. Two residues from the mutagenesis studies are present within 85-99 that reduce GpIba binding when mutated to alanine: $\mathrm{R}^{93}$ and $\mathrm{R}^{97}(68,92)$ (Figure 42 ). While $\mathrm{R}^{97}$ does not appear to interact with GpIb $\alpha$ in the crystal structures, IIa $\mathrm{R}^{93}$ is depicted as being prominent residue in binding to $\mathrm{Y}_{\mathrm{P}}^{279}$ at the C-terminus of both GpIb $\alpha$ crystals $(96,97)$. In addition, the structures display the importance of IIa $\mathrm{N}^{179}$ in binding to GpIb $\alpha \mathrm{D}^{277}$, which is within fragment 173-181 $(96,97)$. Unfortunately, the coverage from the peptic digest does not include a majority of the residues that bind GpIba at ABE-II, such as $\mathrm{R}^{101}, \mathrm{R}^{126}, \mathrm{R}^{165}, \mathrm{R}^{233}, \mathrm{~K}^{235}, \mathrm{~K}^{236}$, and $\mathrm{K}^{240}$ (not shown in Figure 42).

Several studies have found that GpIba binding to IIa has consequences on catalysis. Increases in the rate of IIa catalysis for FXI (23), PAR-1 (67), and GpV (254) are observed when IIa interacts with GpIb $\alpha$. Conversely, IIa bound to GpIb $\alpha$ noncompetitively inhibits the rate of fibrinopeptide A release and catalysis of small chromogenic substrates suggesting an allosteric mechanism transmitted by ABE-II ligand binding to the active site (68). Similar results are described for the ABE-II ligands $\gamma^{\prime}$ peptide (113) and the chondroitin sulfate moiety of thrombomodulin (255). The residues $\mathrm{R}^{97}, \mathrm{E}^{97 \mathrm{~A}}$, and $\mathrm{I}^{174}$ play important roles orienting and binding FpA for catalysis (248) (Figure 42). These residues are within the fragments 85-99 and 173-181 that contain residues involved with binding to GpIb $\alpha$ and display large decreases in \% deuteration. 
Interestingly, $\mathrm{R}^{97}$ does not play a direct role in GpIb $\alpha$ binding to IIa, but when mutated to alanine results in an 8-fold increase of $\mathrm{K}_{\mathrm{D}}(92)$. Therefore, the binding of GpIb $\alpha$ to ABEII is allosterically linked to at least one residue, $\mathrm{R}^{97}$, involved in IIa catalysis of FpA.

As with the $\gamma^{\prime}$ peptide, GpIba is affecting the rate of deuterium exchange for sites distant from ABE-II. Explanations for how allostery is transmitted from ABE-II binding to these regions are discussed in Chapter IV (167). It should be noted that several reports have described allostery resulting from glycocalicin binding to ABE-II. Two ABE-I ligands have been demonstrated to display a reduced affinity for IIa when glycocalicin binds to ABE-II: hirudin (256) and PAR-1 (257). The reduced amount of deuterium exchange for the ABE-I regions 65-84 and 106-116 once a ligand binds to ABE-II illustrates a linkage between the two exosites and supports the observed non-competitive inhibition observed for these ABE-I ligands.

A few important differences exist between GpIb $\alpha$ and the $\gamma^{\prime}$ peptide deuterium protection at 20:1. The first variation is that GpIb $\alpha$ does not affect the autolysis loop's (135-149D) rate of HDX after one minute, whereas the $\gamma^{\prime}$ peptide provides a slight amount of protection. In addition, the GpIb $\alpha$ peptide provides slightly more protection for the A-chain residues -13 to -4 at one minute. These two regions are highly flexible. The fragment -13 to -4 , in addition to being present only in bovine IIa, is not modeled in $\mathrm{X}$-ray structures of bovine IIa binding to the ligands FpA and hirudin $(258,259)$. Thus, these observations for the fragment -13 to -4 may be of little physiologic relevance. With the autolysis loop (135-149D), residue $\mathrm{W}^{141}$ has been postulated to connect ABE-I to the active site (233); however the significance of the $\gamma^{\prime}$ peptide's slight amount of protection for this region may just be a result of stabilizing this pliable loop to a small degree. 
The most striking variation between the two peptides is the shoulder visible at higher $m / z$ for the isotopic cluster representing residues 85-99 when GpIb $\alpha$ interacts with IIa (Figure 36K). This suggests the presence of two different populations of IIa exchanging for deuterium. After ten minutes of deuterium exchange, residues 65-84 still show 1.5 deuterons (-6.7\%) of protection for GpIb $\alpha$. In addition, the fragment 202-207 does not appear to significantly change in the amount of deuterium incorporation compared to one minute of exchange (-15.6\% vs. $-14.1 \%)$. These longer observed effects for these regions coupled with the observation for 85-99 could be a consequence of a small fraction of GpIba binding to ABE-I. Once bound to ABE-I, the interaction may have a negative allosteric effect on ligand binding to ABE-II. Thus, residues 85-99 are no longer protected from deuterium. Such negative allostery between the exosites has been reported in the literature $(70,170)$. In order to test this hypothesis, a series of HDX experiments at 40:1 peptide to protein were performed.

Analysis of HDX Results (II): GpIba (269-286) Interacts with a Unique Site in Thrombin when Compared to the $\gamma^{\prime}$ peptide (410-427). The HDX results for the $\gamma^{\prime}$ peptide at 40:1 demonstrate similar effects on HDX as observed at 20:1 GpIba. A shoulder begins to appear at higher $\mathrm{m} / \mathrm{z}$ for $85-99$ (Figure 36J). An increase in protection for the A-chain residues -13 to -4 at one minute, coupled with sustained protection for 202-207 over ten minutes of exchange are also evident in GpIb $\alpha$ (20:1). However, subtle differences are apparent. Once again, the $\gamma^{\prime}$ peptide slightly protects the autolysis loop after one minute of deuteration. The ABE-I region 65-84 is slightly more protected at one minute at 40:1 than at 20:1. Significant amounts of HDX protection are not observed for 65-84 after ten minutes of exchange at both concentrations of the $\gamma^{\prime}$ peptide. 
A

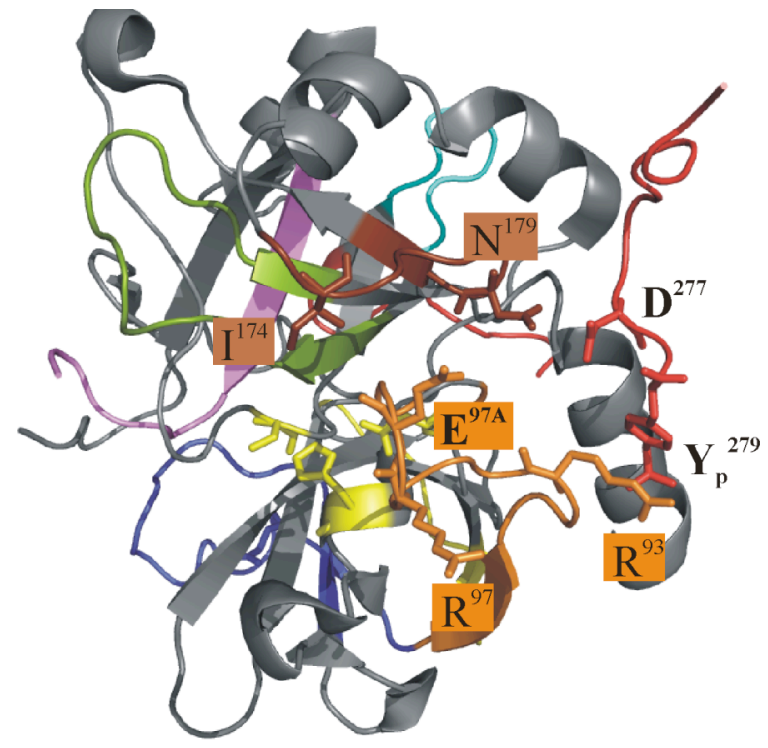

B

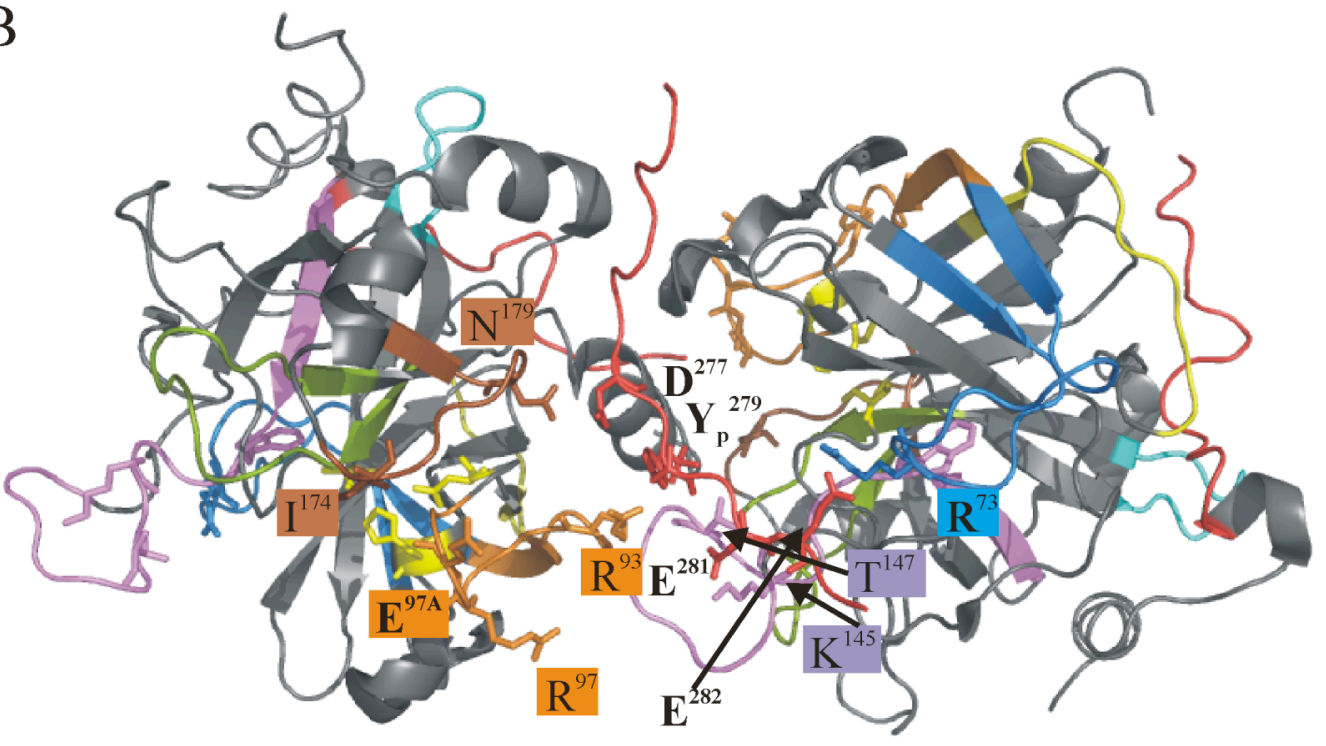

Figure 42: Crystallographic views of GpIba bound to thrombin (II). A. The Dumas structure $(1 \mathrm{P} 8 \mathrm{~V})$ and B. the Celikel structure (1OOK). Regions of HDX coverage are color-coded in both structures: 1H-7 (red), 65-84 (blue), 85-99 (orange), 106-116 (yellow), 135-149D (purple), 173181 (brown), 202-207 (cyan), and 212-227 (green). Residues from the GpIbo peptide that interact with thrombin are labeled without a colored background. Residues from thrombin that bind to GpIb $\alpha$ or are mentioned in the text are labeled with a corresponding colored background. 
These results provide more evidence that these two peptides have slightly different consequences on IIa dynamics when binding to ABE-II. The appearance of a similar shoulder for the $\gamma^{\prime}$ peptide at 40:1 as for GpIb $\alpha$ at 20:1 for residues 85-99 suggest that perhaps higher concentrations of the $\gamma^{\prime}$ peptide could drive the peptide to ABE-I as well. Unfortunately, raising the concentration of the $\gamma^{\prime}$ peptide to $80: 1$ over IIa significantly suppresses peptide ionization in the MALDI-TOF MS. Therefore, further observing the effects of concentration on this interaction was not possible with the HDX technique.

Eight peptide fragments were quantified at 40:1 GpIba to IIa. The non-TCEP digests did not yield any peaks in the mass spectra, thus the peptides -13 to $-4,106-116$, 173-181, and 202-207 were unobserved. These HDX results suggest that the GpIb $\alpha$ peptide is now interacting at ABE-I. Protection for 65-84 is doubled in magnitude from 20:1 (-18.3\%) to $40: 1(-36.2 \%)$. The amount of HDX protection decreases after ten minutes (-12.7). Unexpectedly, the autolysis loop shows quite a dramatic increase in HDX protection at one minute of deuteration (-23.2\%), disappearing within ten minutes.

Examining the Celikel X-ray structure illustrates that several residues within 65-84 and 135-149D are binding to GpIb $\alpha$ in a symmetry related IIa molecule: $\mathrm{R}^{73}-\mathrm{E}^{282}, \mathrm{~K}^{145}$ $\mathrm{E}^{281}$, and $\mathrm{T}^{147}-\mathrm{Y}_{\mathrm{P}}^{279}$ (Figure 42B) (97). These autolysis loop interactions suggest stabilization of this region that is evident in the HDX results as well as in the Celikel structure (97); however, these residues are significantly more disordered in the IIa structures with the ABE-I binding thrombomodulin (260) and hirudin (72). The described interactions are not present in the Dumas structure, which only displays ABE-II binding for GpIba (269-286) and is lacking in electron density to model several of the residues in the autolysis loop (96). 
The ABE-II fragment 85-94, with the ABE-II ligand-binding residue $\mathrm{R}^{93}$, is no longer protected from HDX after one minute. The observed protection for residues 95-99 (within 85-99) may be a result of allostery due to binding at ABE-I, which is also evident with thrombomodulin interactions with IIa's ABE-I (173). Curiously, fragments -3 to 7 and 212-227 are initially protected from HDX at one minute, then dramatically increase in the amount of HDX at ten minutes. In fact, the regions -3 to $7,85-94$, and 212-227 are more exposed to deuterium at ten minutes when compared to IIa alone.

The 40:1 HDX studies suggest that GpIb $\alpha$ no longer interacts specifically at ABEII, but now binds at a unique interface on IIa, perhaps ABE-I. The interaction at the new interface may be weaker than binding at ABE-II. Once bound, the GpIb $\alpha$ peptide can no longer effectively interact at ABE-II; however, the peptide may retain the ability to bind ABE-II through dimerization of IIa. Therefore, the concentration of IIa and GpIb $\alpha$ may have an effect on the type of interaction under study and explain some of the divergent results. The crystal structure reported by Celikel (97) was formed at a total concentration of $25 \mathrm{mg} / \mathrm{ml}$ while the Dumas structure (96) was reported at $10 \mathrm{mg} / \mathrm{ml}$. Unfortunately, the individual concentrations of IIa and GpIb $\alpha$ within the crystallization solutions were not reported. Regardless, the less concentrated Dumas structure (96) may be reflecting the HDX data at 20:1, while the Celikel structure (97) reflective of the 40:1 results.

The same line of thought becomes more interesting as the competitive binding studies determining where GpIb $\alpha$ binds to IIa are considered. One report declares that ABE-I is the destination for GpIb $\alpha$. The investigators used IIa at $0.42 \mathrm{nM}$ and GpIb $\alpha$ at $50 \mu \mathrm{M}$ to monitor the effect of the peptide on catalytic activity, 100,000 times the amount of IIa (93). Conversely, a study proclaiming ABE-II as GpIba's site of interaction 
implemented much lower ratios of IIa $(100 \mathrm{nM})$ to GpIb $\alpha(8 \mu \mathrm{M})$ when performing competitive binding experiments (68). These observations suggest that protein/peptide concentration plays an important role in determining the destination of GpIb $\alpha$ on IIa.

Physiologically, IIa interactions with platelets have been characterized by high affinity binding $\left(\mathrm{K}_{\mathrm{D}}=0.5 \mathrm{nM}\right)$ and intermediate affinity binding $\left(\mathrm{K}_{\mathrm{D}}=50 \mathrm{nM}\right)(261$, 262). An interesting paradox centers on the presence of 25,000 copies of GpIba on the surface of platelets (263), yet they provide only 105-1050 high affinity binding sites for IIa $(101,262)$. The 40:1 HDX data suggest a situation where GpIb $\alpha$ no longer targets ABE-II and instead binds ABE-I. In addition, research has shown that low levels of IIa activate platelets in a GpIb $\alpha$ dependent manner, whereas higher levels of IIa perform this function independent of $\operatorname{GpIb\alpha }(94,264)$. These studies indicate that the binding interface for GpIba on IIa is concentration dependent. It should be noted that IIa interacts with other proteins on the surface of platelets, including PAR-I through ABE-I. As Adam et al. suggests, the relative $\mathrm{K}_{\mathrm{D}}$ and concentrations of all IIa interacting partners must be considered at the site of thrombus formation (265).

Conclusions. These studies further characterize peptide binding to IIa's anion binding exosites. The NMR results suggest that the GpIba peptide binds to IIa through residues $\mathrm{D}^{274}-\mathrm{E}^{285}$ in an extended conformation. These investigations also imply that $\mathrm{Y}$ phosphorylation is not a requirement for effective interaction with IIa. HDX data at 20:1 peptide to IIa demonstrate significant similarities between the GpIb $\alpha$ peptide and the $\gamma^{\prime}$ peptide; however, at higher GpIb $\alpha$ concentrations (40:1), the peptide appears to interact at a new interface. These results suggest that the concentration of the ligand and IIa dictate where the peptide interacts with IIa as well as the corresponding allosteric effects. 


\section{CHAPTER VI}

\section{PERTURBATIONS IN FACTOR XIII RESULTING FROM ACTIVATION AND INHIBITION EXAMINED BY SOLUTION BASED METHODS AND DETECTED BY MALDI-TOF MS}

\section{$\underline{\text { Introduction }}$}

Along with converting fibrinogen to fibrin and activating platelets in preparation for clot formation, thrombin also cleaves the activation peptide of Factor XIII (FXIII) forming FXIIIa. FXIIIa is a transglutaminase that catalyzes the creation of covalent crosslinks between adjacent fibrin molecules leading to the formation of the hard clot. As mentioned in Chapter I, the similarity amongst the X-ray structures of zymogenic (119), IIa activated (118), and $\mathrm{Ca}^{2+}$ activated (117) FXIII suggests two distinct possibilities. First, the activated state of FXIII is not favored during the process of crystallization (117). The other potential explanation is that substrate binding induces the transition to the active conformation of FXIIIa (117).

Previous work by Brian Turner focused on implementing solution-based methods to probe the conformational dynamics of FXIII activation that may be undetected in static crystal structures $(174,175)$. Two useful techniques for observing protein dynamics are HDX coupled with MALDI-TOF MS and differential chemical modification of $\mathrm{C}$ and $\mathrm{K}$ residues (discussed in Chapter III). Figure 43 summarizes previous results using these techniques. HDX studies have revealed potential allostery associated with $\mathrm{Ca}^{2+}$ binding for both the $\beta$ sandwich and $\beta$ barrel 1 domains (174). Furthermore, activating FXIII was 
determined to expose regions within the proposed exosite peptides 4 and 7 (145), to modify $\mathrm{C}^{409}$ at the $\mathrm{A}_{2}$ dimer interface, and to affect the conformation of $\beta$ barrel 2 (174, 175). These studies highlight the subtle conformational changes that are associated with the activation of FXIII.

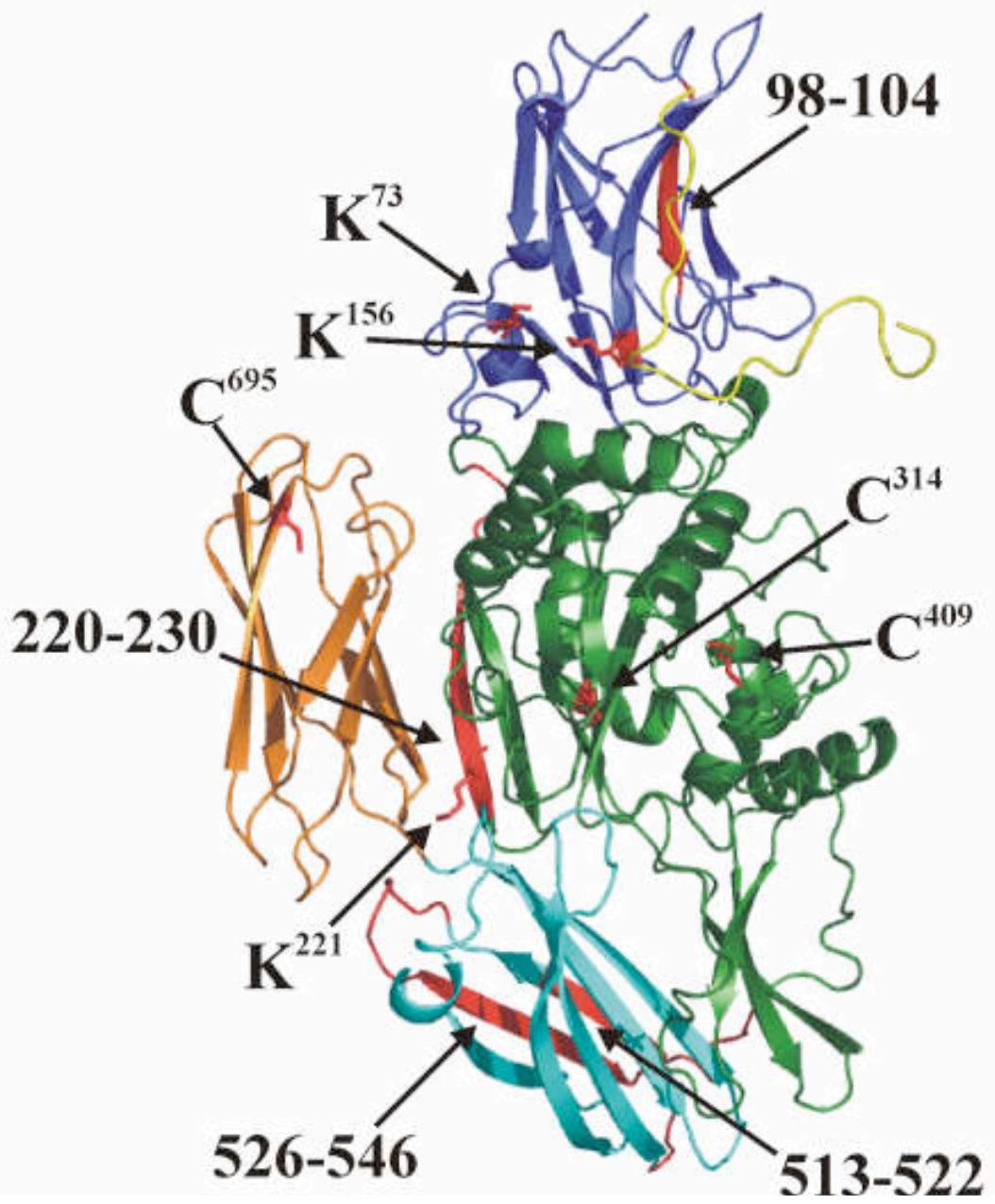

Figure 43: Summary of previous results reported on Factor XIII dynamics $(174,175)$. Residues and peptides experiencing conformational changes upon FXIII activation are highlighted in red. The activation peptide is yellow. $\beta$ sandwich (blue): $1.98-104$ large decrease in $\%$ deuteration upon $\mathrm{Ca}^{2+}$ activation. 2. $\mathrm{K}^{73}$ acetylated within peptide 4 upon $\mathrm{Ca}^{2+}$ activation. 3. $\mathrm{K}^{156}$ acetylated upon IIa activation. Catalytic core (green): 1. 220-230 increase in \% deuteration. 2. $\mathrm{K}^{221}$ acetylated within peptide 7. $3 . \mathrm{C}^{314}$ is alkylated. $4 . \mathrm{C}^{409}$ alkylated at the dimer interface. $\beta$ barrel 1 (cyan): 1. 513-522 small decrease in \% deuteration. 2. 526-546 large decrease in $\%$ deuteration upon $\mathrm{Ca}^{2+}$ activation. $\beta$ barrel 2 (orange): $1 . \mathrm{C}^{695}$ is alkylated. 
The difficulties in crystallizing an active conformer of FXIIIa have led to the prospect of designing an inhibitor for the TGase that may potentially trap the enzyme in the active state. Once irreversibly inhibited, perhaps FXIIIa would be more amenable to crystallization. Recently, Hausch et al. have reported inhibition of tissue TGase 2 with gluten peptide analogs that replace glutamine with the $\mathrm{Q}$ isostere 6-diazo-5-oxonorleucine (DON) (176) (Figure 44A). The DON moiety has now been incorporated into the FXIIIa substrate K9, which is a peptide derived from $\beta$-casein (177). By inhibiting FXIIIa with a peptide (proposed mechanism in Figure 44B (260)), solution based studies could potentially yield clues on how FXIIIa interacts with proteins containing a reactive $\mathrm{Q}$ and how this TGase prepares for $\mathrm{K}$ substrate incorporation. Currently, a crystal structure of an inhibited TGase or a TGase interacting with a ligand has not been solved.

The focus of this chapter is two-fold. First, conformational perturbations in FXIII dynamics were further examined that result from proteolytic activation by IIa (FXIIIa ${ }^{\text {IIa }}$ ) versus nonproteolytic activation with $50 \mathrm{mM} \mathrm{Ca}^{2+}\left(\mathrm{FXIIIa}^{\mathrm{Ca}}\right)$. IIa activation of FXIII led to the increased exposure of residues within peptide 7, a putative FXIIIa exosite. Regarding FXIIIa ${ }^{\mathrm{Ca}}$, unique changes in the dynamics of the $\beta$ sandwich and the $\beta$ barrel 2 may suggest the presence of additional $\mathrm{Ca}^{2+}$ binding sites. The second facet of these studies concerned FXIIIa inhibition. For the first time, the FXIIIa inhibitors iodoacetamide (IAA) and K9 DON were investigated using the described techniques. A TGase activity assay demonstrated that $\mathrm{K} 9$ DON inhibits FXIIIa with an $\mathrm{IC}_{50}$ in the high nM range. HDX reveals that a $\beta$ sandwich fragment and several regions within the $\beta$ barrel 1 are further stabilized by FXIIIa inhibition. Chemical modification shows that K9 DON inhibition impairs the conversion of several residues to the chemically altered state. 
These investigations have demonstrated the efficacy of the DON moiety as an inhibitor specific for FXIIIa. More importantly, potential regions of plasticity resulting from activation and inhibition of FXIIIa have been observed that appear static in the currently available crystal structures.

A

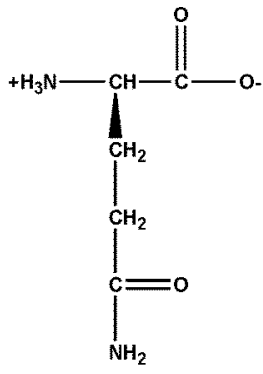

Glutamine

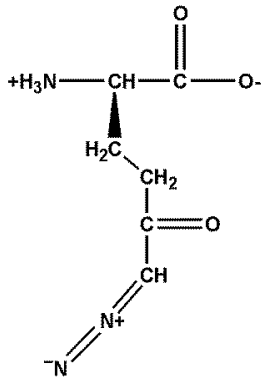

6-diazo-5-oxo-norleucine

B
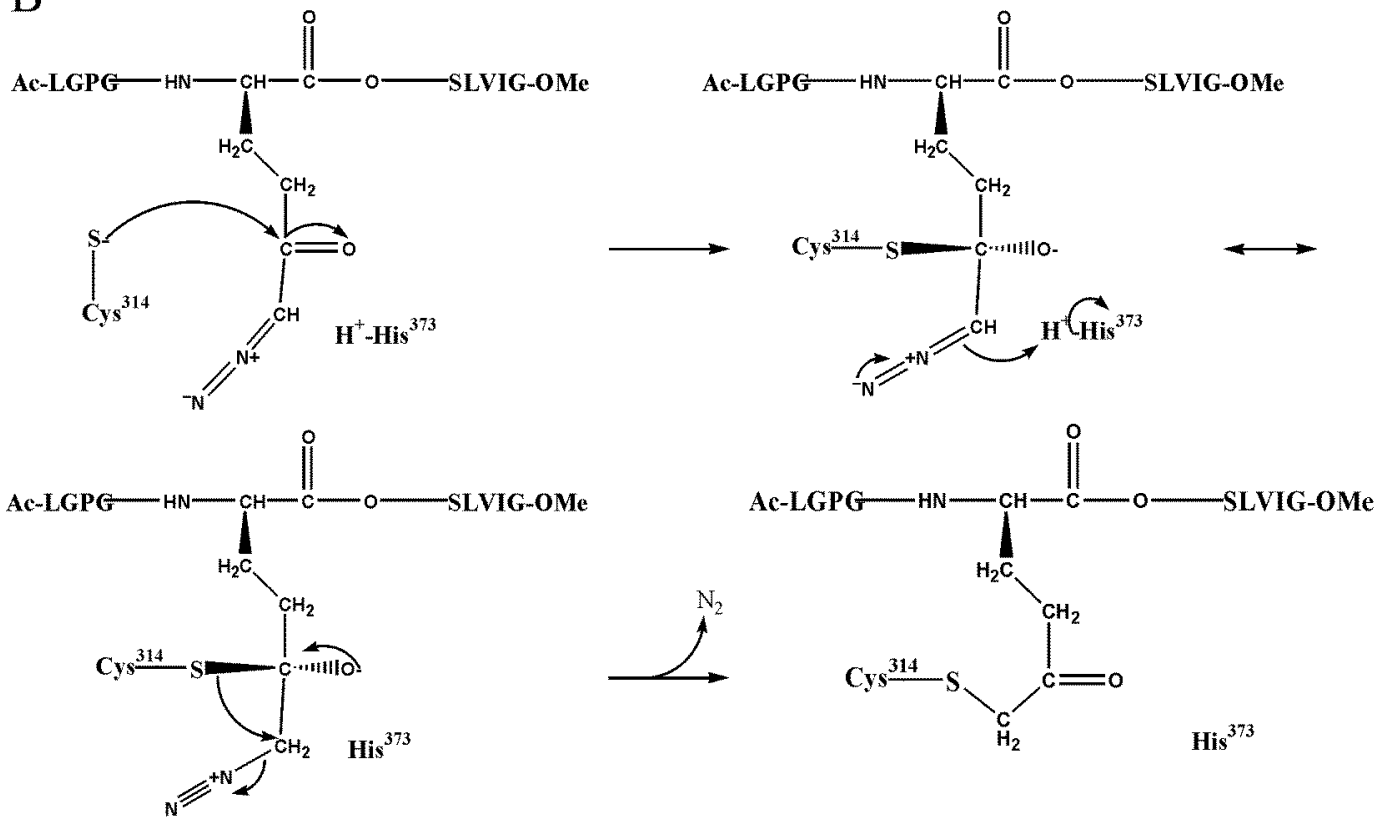

Figure 44: K9 DON Inhibition of FXIIIa. A. The isosteres glutamine and 6-diazo-5-oxonorleucine. B. The proposed mechanism of inhibition proceeds through tetrahedral intermediate formation resulting from cysteine attack at the carbonyl group. The methylene carbon then abstracts a proton from the active site $\mathrm{H}^{373}$. Finally, a thioether is formed resulting in the release of $\mathrm{N}_{2}$ and irreversible inhibition of FXIIIa (266). 


\section{Materials and Methods}

Factor XIII Preparation and Synthetic Peptides. Recombinant human cellular factor XIII (FXIII) was a generous gift from Dr. Paul Bishop (ZymoGenetics, Inc., Seattle, WA) (267). Lyophilized FXIII was reconstituted in $18 \mathrm{M} \Omega$ dI water. The concentration of FXIII was determined with a Cary 100 uv-vis spectrophotometer monitoring absorbance at $280 \mathrm{~nm}$ and using the FXIII extinction coefficient of 1.49 $\mathrm{mL} / \mathrm{mg}-\mathrm{cm}$. Aliquots of $40 \mu \mathrm{M}$ FXIII were stored at $-70{ }^{\circ} \mathrm{C}$.

K9 is a peptide substrate for FXIIIa that is derived from $\beta$-casein (177). The sequence of $\mathrm{K} 9$ is Ac-LGPGQSKVIG-OMe and was synthesized by Peptides International (Louisville, KY). The active site inhibitor Ac-LGPG-(DON)-SLVIG-OMe (K9 DON) was custom synthesized by N-Zyme BioTec GmbH. The K9 DON inhibitor is most soluble at high concentrations in DMSO, therefore this solvent was used to prepare stock solutions. Experiments where $\mathrm{K} 9 \mathrm{DON}$ is absent contain an equivalent amount of DMSO. Peptide concentrations were determined by quantitative amino acid analysis (AAA Service Laboratory, Inc., Boring, OR).

TGase Assay. The Dade-Behring Berichrom Assay (177) utilizes coupled reactions involving FXIIIa and glutamate dehydrogenase (GDH). As FXIIIa reacts with Q from the peptide $\mathrm{K} 9, \mathrm{NH}_{3}$ is released. Next, the primary amine glycine ethyl ester (GEE) concludes the TGase reaction through formation of an isopeptide bond with the K9 peptide. GDH converts $\alpha$-ketoglutarate and $\mathrm{NH}_{3}$ into glutamate with the aid of reducing equivalents from NADH. Thus, FXIIIa activity is directly related to NADH oxidation, which results in decreasing absorbance at $340 \mathrm{~nm}$. 
The FXIII activation mix consisted of $6.9 \mu \mathrm{M}$ FXIII, $30 \mathrm{nM}$ bovine IIa (purified as described in (243)), $1 \mathrm{mM} \mathrm{CaCl}_{2}$, in $20 \mathrm{mM}$ borate, $\mathrm{pH} 8.3$. After $12 \mathrm{~min}$ at $37^{\circ} \mathrm{C}$, IIa activity was inhibited by $376 \mathrm{nM}$ PPACK to prevent further proteolysis of FXIIIa. An additional 12 min at $37^{\circ} \mathrm{C}$ elapsed after which $19 \mu \mathrm{L}$ aliquots of FXIIIa ${ }^{\text {IIa }}$ were made and stored at $-70{ }^{\circ} \mathrm{C}$.

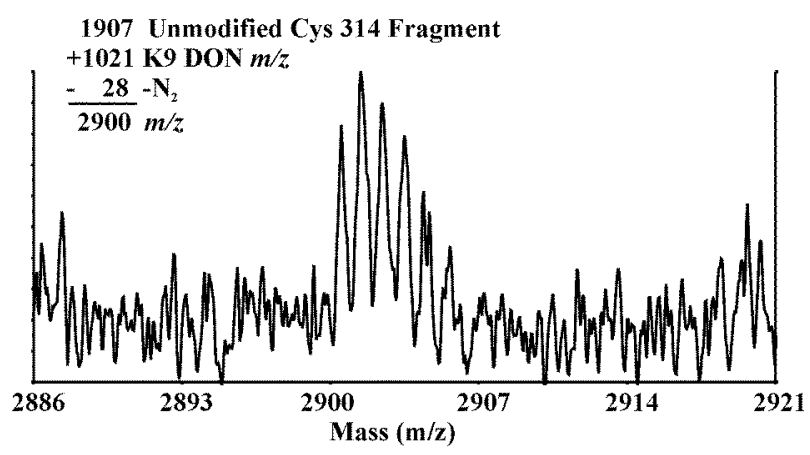

Figure 45: Mass spectrum of the K9 DON modified $\mathrm{C}^{314}$ from a tryptic digest.
Inhibition of FXIIIa $^{\text {IIa }}$ with either K9 DON or iodoacetamide (IAA) was monitored with a modified version of the Berichrom assay (200). Figure 45 displays the mass spectrum indicating K9 DON modification of $C^{314}$ (266). Irreversible inhibition is a time and concentration dependent

phenomenon. Throughout each trial, the volumes of the detector reagent $(250 \mu \mathrm{L})$ and the activator reagent $(163 \mu \mathrm{L})$, as well as FXIIIa ${ }^{\text {IIa }}(218 \mathrm{nM})$ and $\mathrm{K} 9$ peptide $(313 \mu \mathrm{M})$, in $100 \mathrm{mM}$ bicine $\mathrm{pH} 8.3$ were held constant. The amount of the inhibitor K9 DON or IAA ranged from 0 to $5000 \mathrm{nM}$, resulting in inhibitor to FXIIIa active site molar ratios of 1:1 to 23:1. The assay contents were incubated in a Cary $100 \mathrm{uv}$-vis spectrophotometer for 5 min at $37{ }^{\circ} \mathrm{C}$, then the substrate $\mathrm{K} 9$ was added. $\mathrm{NADH}$ oxidation was monitored for 10 min at $340 \mathrm{~nm}$. Since $4 \%$ DMSO is present in the assay mix, a series of control experiments were performed demonstrating that FXIIIa ${ }^{\text {IIa }}$ in $4 \%$ DMSO possesses identical activity to FXIIIa ${ }^{\text {IIa }}$ with an equal amount of dI water (data not shown). 
FXIIIa ${ }^{\text {IIa }}$ activity was measured by determining the slope for the steepest part of the curve. The slope represents the velocity of the reaction $(\Delta \mathrm{abs} / \mathrm{min})$. The resultant slope of FXIIII ${ }^{\mathrm{IIa}}$ activity in the absence of the inhibitor was assigned as $0 \%$ inhibition or $100 \%$ activity. All reaction velocities calculated in the presence of K9 DON or IAA were normalized with respect to the slope observed with $0 \%$ inhibition. As described in Rajagopal et al. (268), $\mathrm{IC}_{50}$ values for inhibition of FXIIIIa ${ }^{\mathrm{IIa}}$ were determined by plotting the $\%$ inhibition versus the log of the inhibitor concentration, which produced an apparent sigmoidal curve. Equation 8 was used to fit the sigmoidal with SigmaPlot and calculate an $\mathrm{IC}_{50}$. In this equation, $\boldsymbol{a}$ represents the minimal curve asymptote, $\boldsymbol{b}$ equals the maximal curve asymptote, and $c$ is the Hill slope which is an indicator of the steepness of the inhibitor-response curve.

$$
\text { \%inhibition }=a+\frac{(b+a)}{1+\left(\frac{\log ([\text { inhibitor }])}{I C_{50}}\right)^{c}}
$$

Activation and Inhibition of FXIII for HDX and NEM Experiments. IIa activation of FXIII proceeded as described for the TGase assay. $\mathrm{Ca}^{2+}$ activation of FXIII was carried out in $50 \mathrm{mM} \mathrm{CaCl}_{2}, 20 \mathrm{mM}$ borate $\mathrm{pH}$ 8.3. The activation mix incubated for 2 hrs at $37^{\circ} \mathrm{C}$. The inhibitors K9 DON or IAA were added at a ten-fold molar excess to FXIIIa and incubated at $37^{\circ} \mathrm{C}$ for $30 \mathrm{~min}$. The amount of inhibitor to FXIIIa (10:1) was chosen based on the results of the TGase assay. Furthermore, the Berichrom assay verified FXIIIa activation and inhibition. As with uninhibited FXIIIa, K9 DON and IAA FXIIIa exhibited limited solubility. For the described experiments, enough FXIIIa is present in solution to gather reliable and reproducible results. 
HDX Sample Preparation. FXIIIa was exchanged into $6.67 \mathrm{mM}$ borate, $0.33 \mathrm{mM}$ $\mathrm{CaCl}_{2}, \mathrm{pH} 8.3$, removing excess inhibitor and/or $\mathrm{Ca}^{2+}$. The exception was the standard for comparison, zymogenic FXIII without $\mathrm{Ca}^{2+}$, which was buffer exchanged into 6.67 $\mathrm{mM}$ borate, $\mathrm{pH}$ 8.3. The concentration of FXIII(a) (the (a) is shorthand for all forms of Factor XIII: unactivated, activated, and inhibited) for all HDX preparations varied from 10-13 $\mu \mathrm{M}$. Aliquots $(36 \mu \mathrm{L})$ of the buffer-exchanged FXIII(a) were evaporated to dryness using a SpeedVac (Savant) and stored at $-70{ }^{\circ} \mathrm{C}$.

HDX Experiments. The HDX protocol is described in more detail in Chapter III. Dry aliquots of FXIII(a) were allowed to come to room temperature before beginning the experiment. $12 \mu \mathrm{L}$ of $99.996 \% \mathrm{D}_{2} \mathrm{O}$ (Cambridge Isotope Laboratories) was added to the aliquot yielding 30-36 $\mu \mathrm{M}$ FXIII(a), $1 \mathrm{mM} \mathrm{CaCl}_{2}$, in $20 \mathrm{mM}$ borate $\mathrm{pH} 8.3$. The samples were incubated in a desiccator at room temperature for 1 or 10 min. Adding $120 \mu \mathrm{L}$ of chilled $0.1 \%$ TFA pH 2.5 quenched HDX. Immediately, the entire quenched solution was transferred to a tube of activated pepsin bound to $6 \%$ agarose (Pierce Chemical). Digestion occurred on ice for $10 \mathrm{~min}$. Centrifugation at $4{ }^{\circ} \mathrm{C}$ separated the digest from the pepsin and $10 \mu \mathrm{L}$ aliquots were immediately frozen in liquid $\mathrm{N}_{2}$ and stored at $-70{ }^{\circ} \mathrm{C}$. Each time point was performed three times.

HDX Analysis. A HDX aliquot of FXIII(a) was thawed, immediately mixed with an equal volume of $10 \mathrm{mg} / \mathrm{mL} \alpha$-cyano-hydroxycinnamic acid matrix ( $\alpha$-CHCA) (Aldrich) in 1:1:1 ethanol/ $\mathrm{CH}_{3} \mathrm{CN} / 0.1 \% \mathrm{TFA}, \mathrm{pH} 2.2$, and $0.5 \mu \mathrm{L}$ spotted onto a chilled stainless steel MALDI plate. The spotted MALDI plate was dried within a SpeedVac unit and then inserted into the MALDI-TOF MS (Voyager DE-Pro, Applied Biosystems). The entire procedure took about $5 \mathrm{~min}$, limiting the amount of back exchange. Spectra 
were collected in the reflector mode over a mass range of 800-3500 m/z with 256 laser shots per spectrum. All peptides in the peptic digest were previously identified by Brian T. Turner, Jr. (174) (Figure 46). Data Explorer (Applied Biosystems) was used to analyze the spectra. Calibration of the spectra involved two singly protonated reference peptides, the monoisotopic mass of 850.4787 Da (residues 535-541) and the quadraisotopic mass of $1375.7097 \mathrm{Da}$ (residues 220 -230). The amount of deuterium uptake by each peptide and the \% difference in deuteration relative to zymogenic FXIII was quantified as described by Sabo et al. (167) and discussed in Chapter III.

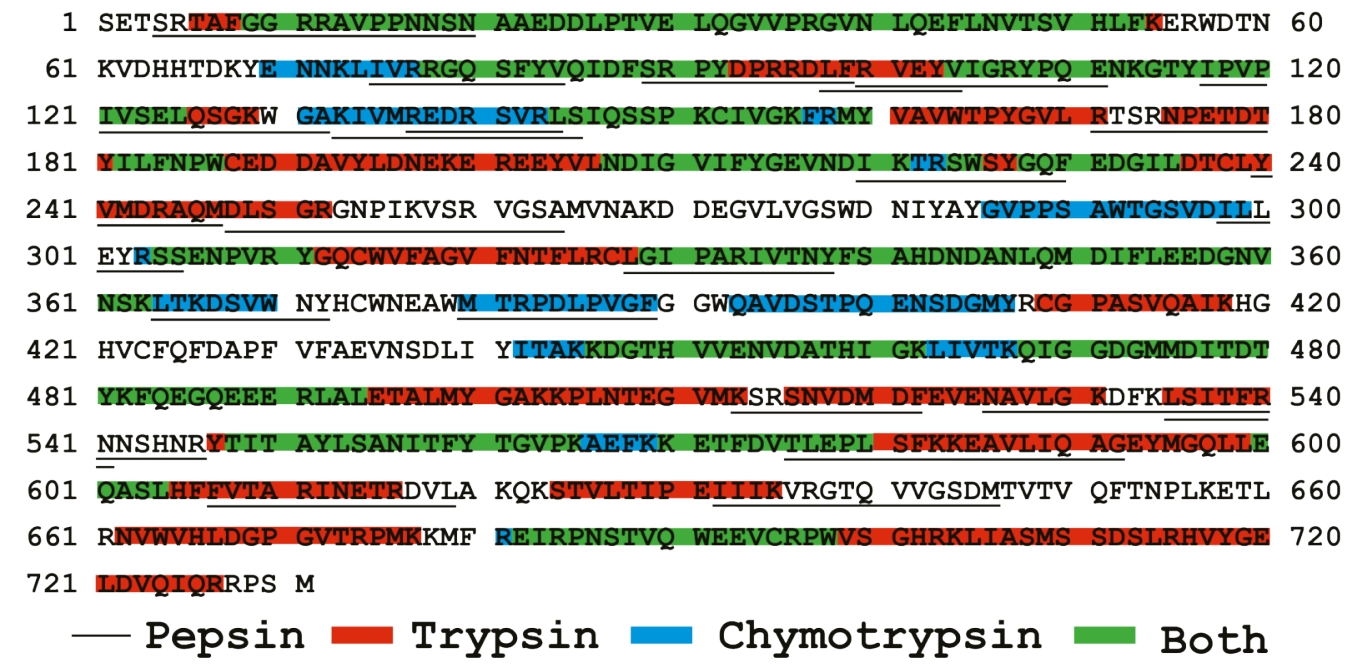

Figure 46: Sequence Coverage Map of FXIII. Pepsin fragments monitored during HDX experiments are underlined (45\% sequence coverage). Residues highlighted in red represent trypsin coverage $(68 \%)$, in blue indicates chymotrypsin coverage $(48 \%)$, and in green displays regions where the proteases overlap (81\%).

Cysteine Modification with NEM. FXIII(a) at $10 \mu \mathrm{M}$ was incubated with $50 \mu \mathrm{M}$ NEM (stock in 50\% $\mathrm{CH}_{3} \mathrm{CN}$ ) for $60 \mathrm{~min}$ at room temperature. The reaction was quenched with the addition of dithiothreitol. In either trypsin buffer $\left(100 \mathrm{mM} \mathrm{NH}_{4} \mathrm{HCO}_{3}\right.$, $\mathrm{pH}$ 8) or chymotrypsin buffer (100 mM Tris-HCl, $\left.10 \mathrm{mM} \mathrm{CaCl}_{2}, \mathrm{pH} 7.4\right)$, the quenched samples were digested with $2 \mu \mathrm{L}$ of $1 \mathrm{mg} / \mathrm{mL}$ chymotrypsin $\left(25^{\circ} \mathrm{C}\right)$ or $1 \mathrm{mg} / \mathrm{mL}$ trypsin 
$\left(37^{\circ} \mathrm{C}\right)$ for at least $4 \mathrm{hr}$. Proteolysis was quenched by acidification with $5 \%$ TFA. Chymotrypsin cleaves primarily after the aromatic residues W, Y, and F. Trypsin hydrolysis occurs primarily after the positively charged $\mathrm{R}$ and $\mathrm{K}$ residues.

Lysine Acetylation and Limited Proteolysis Experiments. In $10 \mathrm{mM} \mathrm{NH}_{4} \mathrm{HCO}_{3} \mathrm{pH}$ 7.4, FXIII at $10 \mu \mathrm{M}$ was either activated with $\mathrm{Ca}^{2+}$ or with IIa. Inhibition of FXIIIa occurred concurrently with the activation process in the presence of $20 \mu \mathrm{M}$ IAA or K9 DON in DMSO. $\mathrm{Ca}^{2+}$ activation occurred in $50 \mathrm{mM} \mathrm{CaCl}$ for $2 \mathrm{hr}$ at $37{ }^{\circ} \mathrm{C}$. IIa activation took place with $42 \mathrm{nM}$ IIa and $1 \mathrm{mM} \mathrm{CaCl}_{2}$ for 12 min at $37^{\circ} \mathrm{C}$. PPACK was used to inhibit IIa activity and the FXIIIa mix incubated for an additional 108 min.

After 2 hours, $5 \mu \mathrm{L}$ of $600 \mathrm{mM}$ acetic anhydride (AA) in DMF was added to FXIII(a) and allowed to incubate for $1 \mathrm{hr}$. The acetylation mix was acidified with $1 \%$ TFA. Residual AA and the byproduct acetic acid were removed by drying in a SpeedVac concentrator device. When performing limited proteolysis, AA was not added but FXIII(a) was handled in a similar manner. The dry protein was reconstituted in dI water. The modified protein was then split in half and addition of buffer yielded $10 \mu \mathrm{M}$ FXIII(a) in $100 \mathrm{mM} \mathrm{NH}_{4} \mathrm{HCO}_{3} \mathrm{pH} 8.0$ (trypsin) or in $100 \mathrm{mM}$ Tris- $\mathrm{HCl}, 10 \mathrm{mM} \mathrm{CaCl} 2 \mathrm{pH} 7.4$ (chymotrypsin). To $25 \mu \mathrm{L}$ of the modified protein in digestion buffer, $2 \mu \mathrm{L}$ of $1 \mathrm{mg} / \mathrm{ml}$ trypsin $\left(37^{\circ} \mathrm{C}\right)$ or chymotrypsin $\left(25^{\circ} \mathrm{C}\right)$ was added and proteolysis occurred for $4 \mathrm{hr}$. With limited proteolysis, the digest was quenched after 30 min with $5 \%$ TFA.

MALDI-TOF Mass Spectrometry for Chemical Modification and Limited Proteolysis. C18 ZipTips (Microcon) were used to concentrate and clean up the digested FXIII(a) samples with elution into $50 \% \mathrm{CH}_{3} \mathrm{CN}$. The eluted peptides were mixed with 5 $\mu \mathrm{L}$ of either $\alpha$-CHCA or ferulic acid MALDI matrix. The samples were spotted on a 
stainless steel MALDI plate and acquired in reflector mode using MALDI-TOF MS. The mass range was from $550-4000 \mathrm{~m} / \mathrm{z}$ and 256 shots per spectrum were collected. At least three separate trials were performed for each state of FXIII(a) monitored and at least two spectra collected for each trial. All spectra were analyzed as described by Turner et al (175) and the FXIII sequence coverage produced with chymotrypsin and trypsin is presented in Figure 46. For limited proteolysis, the intensity of a monoisotopic peak representing FXIII(a) digest fragments was normalized with respect to the most intense (abundant) monoisotopic peak. Differences in relative peak intensities greater than $20 \%$ were considered significant for the limited proteolysis studies based on a comparative standard employed by Ansong et al. (218).

\section{Results}

TGase Assay with FXIIII ${ }^{I I a}$. A modified version of the Dade-Behring Berichrom assay was performed to determine the potency of the irreversible inhibitor K9 DON for FXIIIa ${ }^{\text {IIa }}$ and the results compared to IAA. Figure $47 \mathrm{~A}$ displays a series of progress curves for inhibition of FXIIIa ${ }^{\mathrm{IIa}}$ with increasing concentrations of K9 DON and Figure 47B presents a plot of the data fitted to the Equation 8 . The calculated $\mathrm{IC}_{50}$ was $816 \pm 32$ nM. The small molecular inhibitor IAA was slightly more effective with an $\mathrm{IC}_{50}$ of $539 \pm$ $74 \mathrm{nM}$. The $\mathrm{p}$ value comparing the two inhibitors was $\mathrm{p}=0.03$. Briefly, the $\mathrm{p}$ value is a statistical tool gauging the probability that the observed difference between two values can be attributed to chance alone. Typically, $\mathrm{p}$ values less than 0.05 are considered to reflect significant variance amongst two data sets.

Hydrogen Deuterium Exchange Coupled with MALDI-TOF Mass Spectrometry. The solvent accessibility of IAA or K9 DON inhibited FXIIIa was compared to the 
zymogen, FXIIIa ${ }^{\mathrm{Ca}}$, and FXIIIa ${ }^{\mathrm{IIa}}$. Table 10 contains the $\%$ differences in HDX for FXIIIa relative to the zymogen. The table groups the data according to the activation method observed. The amount of deuterium incorporated at each time point is presented in Figure $48\left(\right.$ FXIIIa $\left.^{\mathrm{Ca}}\right)$ and Figure 49 (FXIIIa $\left.{ }^{\mathrm{IIa}}\right)$ as well as in Table 11.

Figure 47: TGase Assay and FXIIIa inhibition by K9 DON. A. Eight separate trials were performed at varying inhibitor concentrations: FXIIIa in the absence of K9 DON (navy blue); $250 \mathrm{nM}$ K9 DON (red); $400 \mathrm{nM}$ K9 DON (orange); 700 nM K9 DON (pink); 900 nM K9 DON (purple); $1500 \mathrm{nM}$ K9 DON (brown); 2500 nM K9 DON (cyan); and 5000 nM K9 DON (blue). The assay components, FXIIIa, and the indicated amount of inhibitor were incubated for $5 \mathrm{~min}$ at $37^{\circ} \mathrm{C}$, at which point the substrate K9 was introduced to the assay mix. The graph depicts the oxidation of $\mathrm{NADH}$ as a function of time. B. A plot of $\%$ inhibition versus the $\log$ of inhibitor concentration. Open and filled in circles represent IAA and K9 DON, respectively. Three separate trials were performed and the plot was fit to equation 8 . The $\mathrm{IC}_{50}$ was determined as $815.5 \pm 31.9 \mathrm{nM}$ for $\mathrm{K} 9 \mathrm{DON}$ and $538.8 \pm 74.3 \mathrm{nM}$ for IAA.

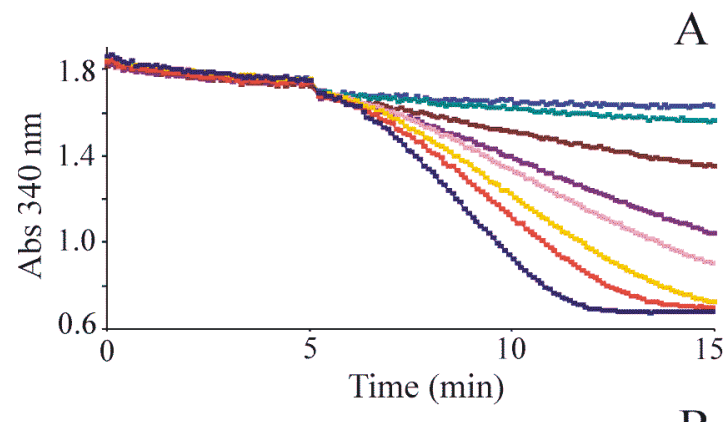

A

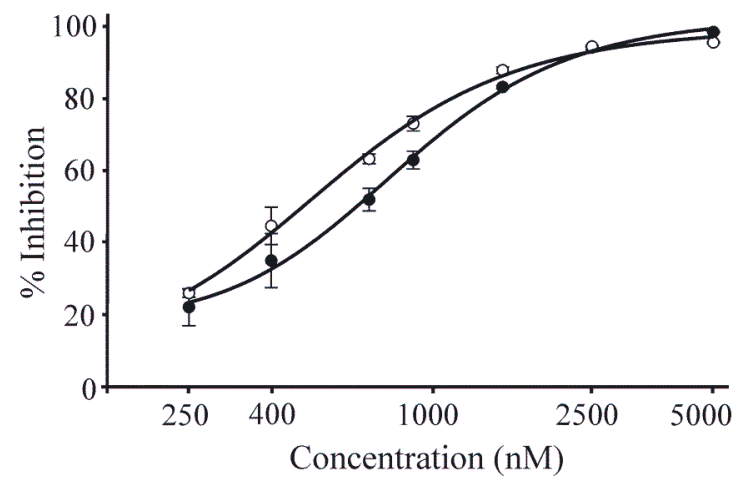

The $\beta$-sandwich residues 98-104 were only present in the FXIIIa ${ }^{\mathrm{Ca}}$ spectra, presumably due to pepsin's inability to produce this peptide from FXIIIa ${ }^{\text {IIa }}$. Inhibition of FXIIIa $^{\mathrm{Ca}}$ with $\mathrm{K} 9 \mathrm{DON}$ resulted in differences in the deuteration profiles for this peptide at one and ten minutes. Whereas FXIIIa $^{\mathrm{Ca}}$ displayed an increase in solvent accessibility after 10 minutes (from $-24.3 \%$ at one minute to $-15.3 \%$ after ten minutes), K9 DON FXIIIa $^{\mathrm{Ca}}$ was observed to acquire less deuterium over a similar time span (from $-15.5 \%$ at one minute to $-30.1 \%$ after ten minutes). As for IAA FXIIIa ${ }^{\mathrm{Ca}}$, inhibition with the small molecular inhibitor leads to sustained stabilization over ten minutes of HDX $(-31.9 \%$ at one minute to $-35.0 \%$ after ten minutes). 
Within the catalytic core, several fragments were observed as displaying similar differences in deuterium exchange, regardless of the FXIIIa state monitored. For residues 220-230, deuterium incorporation varied from 4.5-7.0\% more exposure relative to the zymogen at one minute and at ten minutes the range was $6.2-9.7 \%$. The only exception was with IAA FXIIIa ${ }^{\mathrm{Ca}}$ where one minute of exposure to deuterium did not yield a significant difference in deuteration when compared to the zymogen (1.7\%). Similarly, the results for residues 240-247 were within the standard deviation for all FXIIIa conditions examined: one minute, $6.0-7.3 \%$ and ten minutes, 5.9-10.4\%. Only FXIIIa ${ }^{\text {IIa }}$ presented reliable isotopic clusters for quantification of 248-264. Once again, the type of inhibition when compared to FXIIIa ${ }^{\text {IIa }}$ had little effect on deuterium exchange. In this case, significant protection was observed initially at one minute (5.9\%-6.7\%) and within ten minutes, the exchange began to approach zymogenic FXIII (2.2\%-3.1\%).

\begin{tabular}{|c|c|c|c|c|c|c|c|}
\hline \multirow{2}{*}{ Residues } & \multirow{2}{*}{$\begin{array}{l}\text { Theo. } \\
\mathrm{D}_{\text {max }}{ }^{\circ}\end{array}$} & \multicolumn{2}{|c|}{ FXIIIa $^{\mathrm{Ca}}$} & \multicolumn{2}{|c|}{ K9 DON FXIIIa ${ }^{\mathrm{Ca}}$} & \multicolumn{2}{|c|}{ IAA FXIIIa $^{\mathrm{Ca}}$} \\
\hline & & $1 \mathrm{~m}$ & $10 \mathrm{~m}$ & $1 \mathrm{~m}$ & $10 \mathrm{~m}$ & $1 \mathrm{~m}$ & $10 \mathrm{~m}$ \\
\hline 98-104 & 6.5 & $-24.3^{c}$ & -15.3 & -15.5 & -30.1 & -31.9 & -35.0 \\
\hline $220-230$ & 10.8 & 5.4 & 9.7 & 7.0 & 7.3 & 1.7 & 6.2 \\
\hline $240-247$ & 7.5 & 6.0 & 7.1 & 7.2 & 5.9 & 6.9 & 8.5 \\
\hline $513-522$ & 9.7 & -11.6 & -10.6 & -10.0 & -11.4 & -15.6 & -15.6 \\
\hline $526-546$ & 21.2 & -24.1 & -25.8 & $\mathrm{n} / \mathrm{a}^{d}$ & -32.7 & $\mathrm{n} / \mathrm{a}$ & $\mathrm{n} / \mathrm{a}$ \\
\hline $535-541$ & 6.5 & 1.8 & 2.4 & 2.6 & 2.7 & 1.3 & 4.2 \\
\hline \multirow{2}{*}{ Residues } & \multirow{2}{*}{$\begin{array}{l}\text { Theo. } \\
\mathrm{D}_{\max }\end{array}$} & \multicolumn{2}{|c|}{ FXIIIa $^{\text {IIa }}$} & \multicolumn{2}{|c|}{ K9 DON FXIIIa ${ }^{\text {IIa }}$} & \multicolumn{2}{|c|}{ IAA FXIIIa $^{\text {IIa }}$} \\
\hline & & $1 \mathrm{~m}$ & $10 \mathrm{~m}$ & $1 \mathrm{~m}$ & $10 \mathrm{~m}$ & $1 \mathrm{~m}$ & $10 \mathrm{~m}$ \\
\hline $220-230$ & 10.8 & 4.5 & 7.2 & 5.9 & 6.3 & 6.6 & 7.5 \\
\hline $240-247$ & 7.5 & 6.0 & 6.8 & 6.5 & 6.7 & 7.3 & 10.4 \\
\hline $248-264$ & 15.9 & 6.0 & 3.1 & 5.9 & 2.2 & 6.7 & $\mathrm{n} / \mathrm{a}$ \\
\hline $513-522$ & 9.7 & -5.4 & -5.3 & -7.3 & -10.3 & -8.0 & -9.4 \\
\hline $526-546$ & 21.2 & -2.6 & -2.4 & -10.0 & -11.8 & -4.0 & -9.0 \\
\hline $535-541$ & 6.5 & 1.8 & 4.2 & 2.8 & 4.9 & 2.3 & 5.1 \\
\hline \multicolumn{8}{|c|}{$\begin{array}{l}{ }^{a} \text { The } \% \text { change for a particular peptide is calculated by Equation } 7 \text { (Chapter III). }{ }^{b} \text { The maximum } \\
\text { number of exchangeable protons within the indicated peptide, assuming } 100 \% \text { deuteration. } \\
\text { (Chapter III). A fully deuterated peptide would theoretically have acquired this amount of } \\
\text { deuterons. }{ }^{c} \text { The values in bold represent significant changes in deuteration of greater than } \pm 4.5 \% \text {. } \\
{ }^{d} \mathrm{n} / \mathrm{a} \text { refers to a peptide that was either not observed in the peptic digest or was not of sufficient } \\
\text { intensity to quantify. }\end{array}$} \\
\hline
\end{tabular}




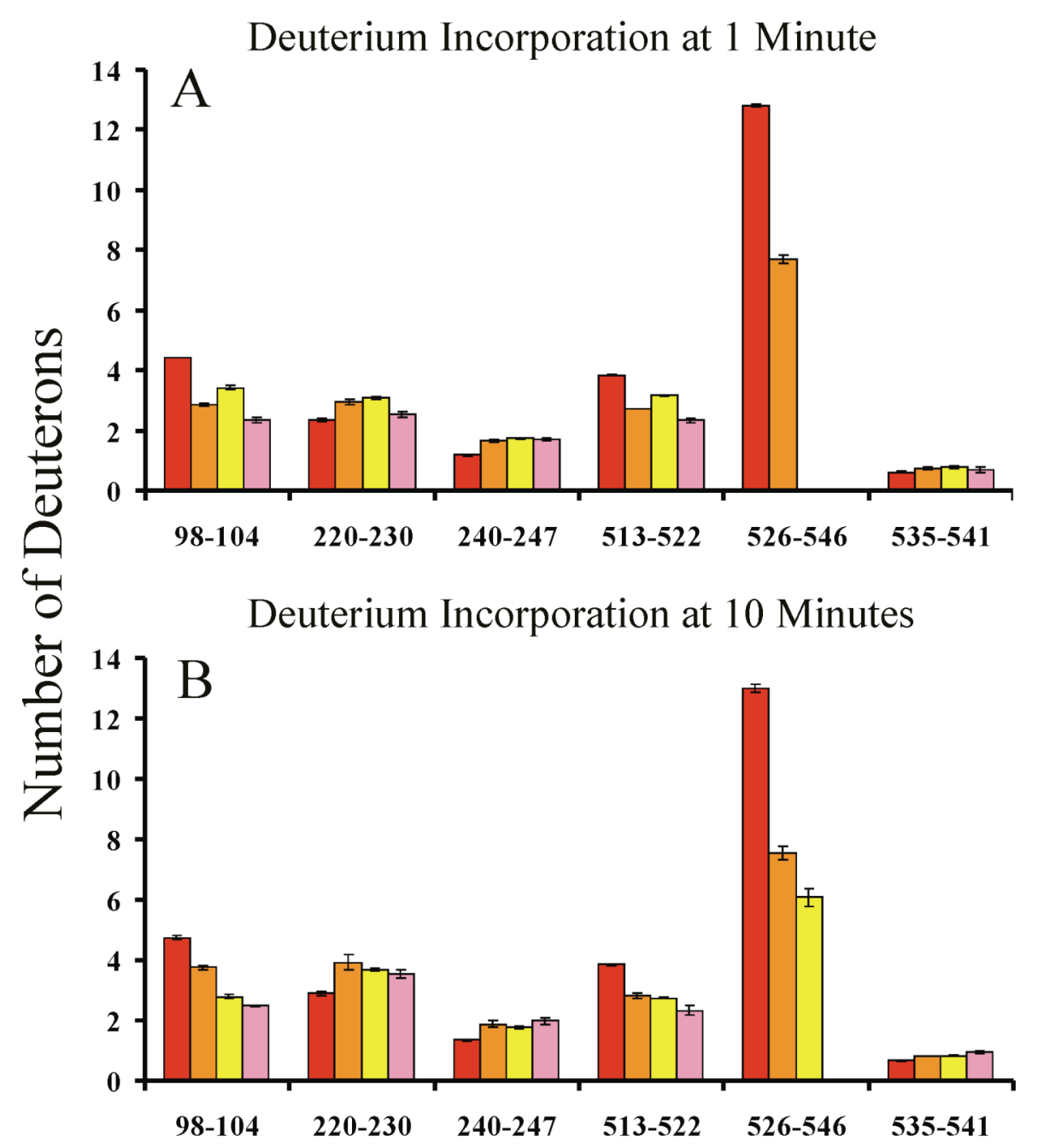

Figure 48: Graphs of deuterium incorporation for FXIIIa ${ }^{\mathrm{Ca}}$. A. $1 \mathrm{~min}$ and B. $10 \mathrm{~min}$ of HDX. The bars in the graph correspond to the following conditions: zymogenic FXIII (red), FXIIIa ${ }^{\mathrm{Ca}}$ (orange), K9 DON FXIIIa ${ }^{\mathrm{Ca}}$ (yellow), and IAA FXIIIa ${ }^{\mathrm{Ca}}$ (pink). Errors correspond to standard deviation of the mean for three independent trials.

The final region of FXIII investigated with HDX was the $\beta$ barrel 1. Considering all FXIIIa $^{\mathrm{Ca}}$ states, two of the three regions observed, 513-522 (-10.0\% to $\left.-15.6 \%\right)$, and $535-541(1.3 \%$ to $4.2 \%)$, were similar in regard to the dynamics of deuterium exchange at one and ten minutes. However, the presence of the K9 DON peptide protects the fragment $526-546$ by $6.9 \%$ after ten minutes of $\mathrm{HDX}\left(-25.8 \%\right.$ for $\mathrm{FXIIIa}^{\mathrm{Ca}}$ and $-32.7 \%$ for $\mathrm{K} 9 \mathrm{DON}$ FXIIIa ${ }^{\mathrm{Ca}}$ ). Unfortunately, this region was unable to be observed in the IAA FXIIIa $^{\mathrm{Ca}}$ spectra (Tables 10 and 11). 


\begin{tabular}{|c|c|c|c|c|c|c|c|c|}
\hline \multirow[b]{2}{*}{ Peptide } & \multicolumn{8}{|c|}{ Table 11: Deuterium Incorporation for FXIII } \\
\hline & $\begin{array}{l}\text { Theo. } \\
D_{\max }\end{array}$ & Zymogen & $\mathrm{FXIIIa}^{\mathrm{Ca}}$ & $\begin{array}{l}\text { K9 DON } \\
\text { FXIIIa }^{\mathrm{Ca}}\end{array}$ & $\begin{array}{c}\text { IAA } \\
\text { FXIIIa }\end{array}$ & FXIIIa $^{\text {IIa }}$ & $\begin{array}{l}\text { K9 DON } \\
\text { FXIIIa }^{\text {IIa }}\end{array}$ & $\begin{array}{c}\text { IAA } \\
\text { FXIIIa }^{\text {IIa }} \\
\end{array}$ \\
\hline $98-104$ & 6.5 & $4.4 \pm 0.0$ & $2.9 \pm 0.1$ & $3.4 \pm 0.1$ & $2.4 \pm 0.1$ & $\mathrm{n} / \mathrm{a}$ & $\mathrm{n} / \mathrm{a}$ & $\mathrm{n} / \mathrm{a}$ \\
\hline $220-230$ & 10.8 & $2.4 \pm 0.1$ & $2.9 \pm 0.1$ & $3.1 \pm 0.1$ & $2.5 \pm 0.1$ & $2.8 \pm 0.1$ & $3.0 \pm 0.0$ & $3.1 \pm 0.1$ \\
\hline $240-247$ & 7.5 & $1.2 \pm 0.0$ & $1.7 \pm 0.1$ & $1.7 \pm 0.0$ & $1.7 \pm 0.1$ & $1.6 \pm 0.0$ & $1.7 \pm 0.0$ & $1.7 \pm 0.1$ \\
\hline $248-264$ & 15.9 & $2.9 \pm 0.0$ & $\mathrm{n} / \mathrm{a}^{b}$ & $\mathrm{n} / \mathrm{a}$ & $\mathrm{n} / \mathrm{a}$ & $3.8 \pm 0.0$ & $3.8 \pm 0.0$ & $3.9 \pm 0.1$ \\
\hline 513-522 & 9.7 & $3.8 \pm 0.0$ & $2.7 \pm 0.0$ & $2.9 \pm 0.0$ & $2.3 \pm 0.1$ & $3.3 \pm 0.1$ & $3.1 \pm 0.0$ & $3.1 \pm 0.2$ \\
\hline $526-546$ & 21.2 & $12.8 \pm 0.1$ & $7.7 \pm 0.2$ & $\mathrm{n} / \mathrm{a}$ & $\mathrm{n} / \mathrm{a}$ & $12.3 \pm 0.1$ & $10.7 \pm 0.3$ & $12.0 \pm 0.5$ \\
\hline $535-541$ & 6.5 & $0.6 \pm 0.0$ & $0.7 \pm 0.1$ & $0.8 \pm 0.1$ & $0.7 \pm 0.1$ & $0.7 \pm 0.1$ & $0.8 \pm 0.1$ & $0.8 \pm 0.1$ \\
\hline \multicolumn{9}{|c|}{ Ten Minutes } \\
\hline Peptide & $\begin{array}{l}\text { Theo. } \\
\mathrm{D}_{\max }\end{array}$ & Zymogen & $\mathrm{FXIIIa}^{\mathrm{Ca}}$ & $\begin{array}{l}\text { K9 DON } \\
\text { FXIIIa }^{\mathrm{Ca}}\end{array}$ & $\underset{\text { FXIIIIa }}{\text { IAa }}$ & FXIIIa $^{\text {IIa }}$ & $\begin{array}{l}\text { K9 DON } \\
\text { FXIIIa }^{\text {IIa }}\end{array}$ & $\begin{array}{c}\text { IAA } \\
\text { FXIIIIa }^{\text {IIa }}\end{array}$ \\
\hline $98-104$ & 6.5 & $4.8 \pm 0.1$ & $3.8 \pm 0.1$ & $2.8 \pm 0.1$ & $2.5 \pm 0.0$ & $\mathrm{n} / \mathrm{a}$ & $\mathrm{n} / \mathrm{a}$ & $\mathrm{n} / \mathrm{a}$ \\
\hline $220-230$ & 10.8 & $2.9 \pm 0.1$ & $3.9 \pm 0.3$ & $3.7 \pm 0.1$ & $3.6 \pm 0.2$ & $3.7 \pm 0.0$ & $3.6 \pm 0.1$ & $3.7 \pm 0.1$ \\
\hline $240-247$ & 7.5 & $1.4 \pm 0.0$ & $1.9 \pm 0.1$ & $1.8 \pm 0.0$ & $2.0 \pm 0.1$ & $1.9 \pm 0.0$ & $1.9 \pm 0.1$ & $2.1 \pm 0.2$ \\
\hline $248-264$ & 15.9 & $3.5 \pm 0.0$ & $\mathrm{n} / \mathrm{a}$ & $\mathrm{n} / \mathrm{a}$ & $\mathrm{n} / \mathrm{a}$ & $4.0 \pm 0.1$ & $3.9 \pm 0.0$ & $\mathrm{n} / \mathrm{a}$ \\
\hline $513-522$ & 9.7 & $3.9 \pm 0.0$ & $2.8 \pm 0.1$ & $2.7 \pm 0.0$ & $2.3 \pm 0.2$ & $3.3 \pm 0.0$ & $2.9 \pm 0.3$ & $2.9 \pm 0.0$ \\
\hline $526-546$ & 21.2 & $13.0 \pm 0.1$ & $7.5 \pm 0.2$ & $6.1 \pm 0.3$ & $\mathrm{n} / \mathrm{a}$ & $12.5 \pm 0.2$ & $10.5 \pm 0.8$ & $11.0 \pm 0.2$ \\
\hline 535-541 & 6.5 & $0.7 \pm 0.0$ & $0.8 \pm 0.0$ & $0.8 \pm 0.0$ & $0.9 \pm 0.1$ & $0.9 \pm 0.0$ & $1.0 \pm 0.0$ & $1.0 \pm 0.1$ \\
\hline
\end{tabular}

When considering the various forms of FXIIIa ${ }^{\text {IIa }}$ (Tables 10 and 11), residues 513522 experience slightly more protection from solvent in both inhibited forms of FXIIIa ${ }^{\text {IIa }}$. At ten minutes, FXIIIa ${ }^{\text {IIa }}$ had incorporated 3.3 deuterons $(-5.3 \%)$ and both types of inhibited FXIIIa ${ }^{\text {IIa }}$ integrated 2.9 deuterons $(-9.4 \%$ and $-10.3 \%)$. The amount of deuteration in FXIIIa ${ }^{\mathrm{IIa}}$ for 526-546 was comparable to FXIII (-2.4\% to $\left.-2.6 \%\right)$. For the same span of residues, K9 DON FXIIIa ${ }^{\text {IIa }}$ exhibited an immediate decrease in solvent accessibility after 1 minute $(-10.0 \%)$; conversely, IAA FXIIIa ${ }^{\text {IIa }}$ affected the dynamics of HDX in a gradual manner, reaching a statistically significant difference following ten minutes of HDX (-9.0\%). Lastly, the fragment 535-541 became more exposed to solvent at ten minutes in all forms of FXIIIa ${ }^{\mathrm{IIa}}$ (4.2\% to $\left.5.1 \%\right)$. 


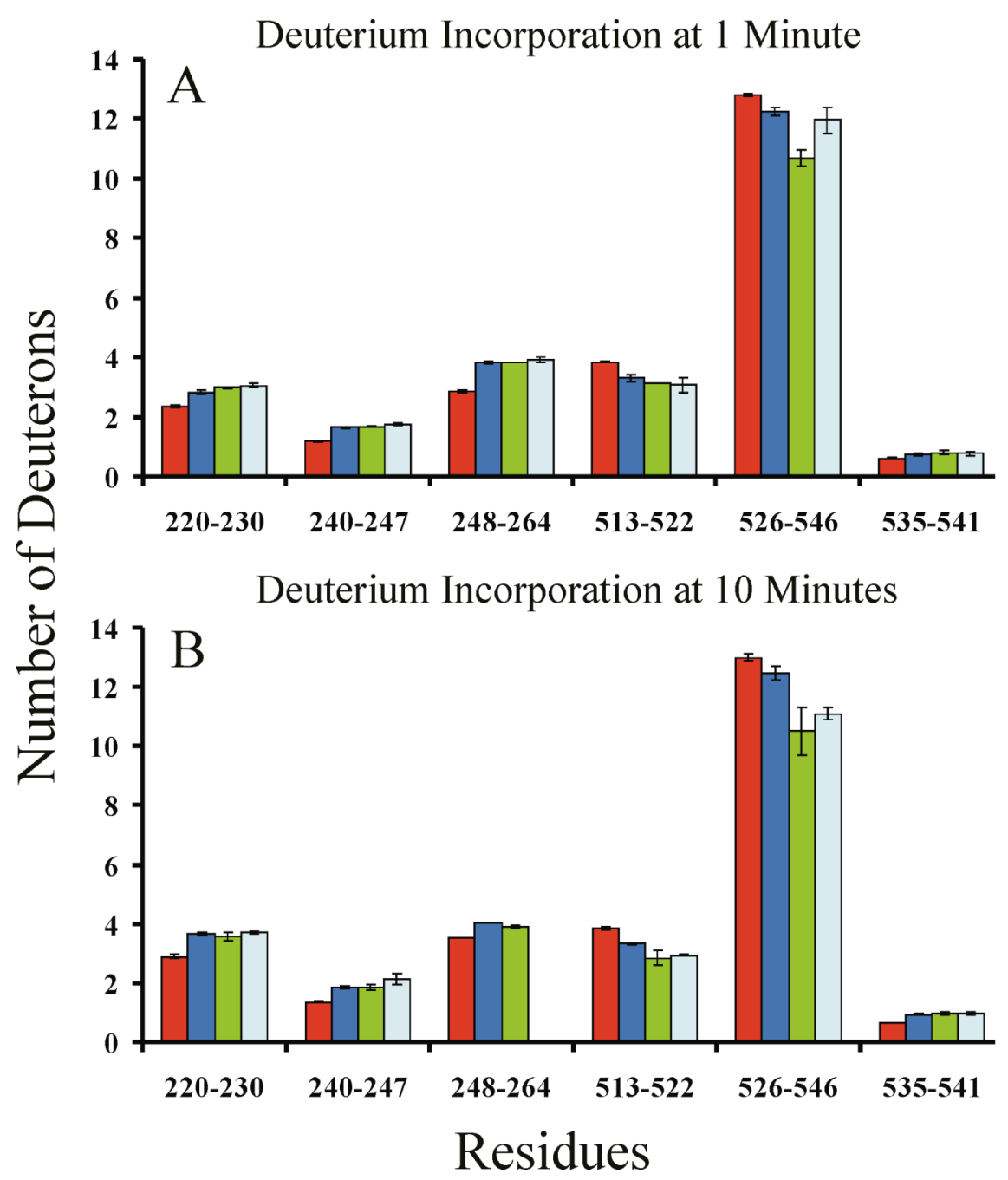

Figure 49: Graphs of deuterium incorporation for FXIIIa ${ }^{\text {IIa }}$. A. 1 min and B. 10 min of HDX. The bars in the graph correspond to the following conditions: zymogenic FXIII (red), FXIIIa ${ }^{\text {IIa }}$ (blue), K9 DON FXIIIa ${ }^{\text {IIa }}$ (green), and IAA FXIIIa ${ }^{\text {IIa }}$ (cyan). Errors correspond to standard deviation of the mean for three independent trials.

The isotopic clusters representing 526-546 for FXIIIa ${ }^{\mathrm{Ca}}$ and K9 DON FXIIIa ${ }^{\mathrm{Ca}}$ were of an uneven distribution with a small rise in peak intensity toward higher $m / z$ (Figure 50). This observation led to a separate series of HDX experiments to be performed on the zymogen in the presence of $1 \mathrm{mM} \mathrm{Ca}^{2+}$. The rationale for these HDX trials was to differentiate the effects of $\mathrm{Ca}^{2+}$ concentration on deuterium exchange. Figure 50 shows an expanded isotopic cluster for FXIII in $1 \mathrm{mM} \mathrm{Ca}^{2+}$, intermediate between the zymogen and FXIIIa ${ }^{\mathrm{Ca}}$, as well as, the spectra for the states of FXIIIa ${ }^{\text {IIa }}$ in the same region. 

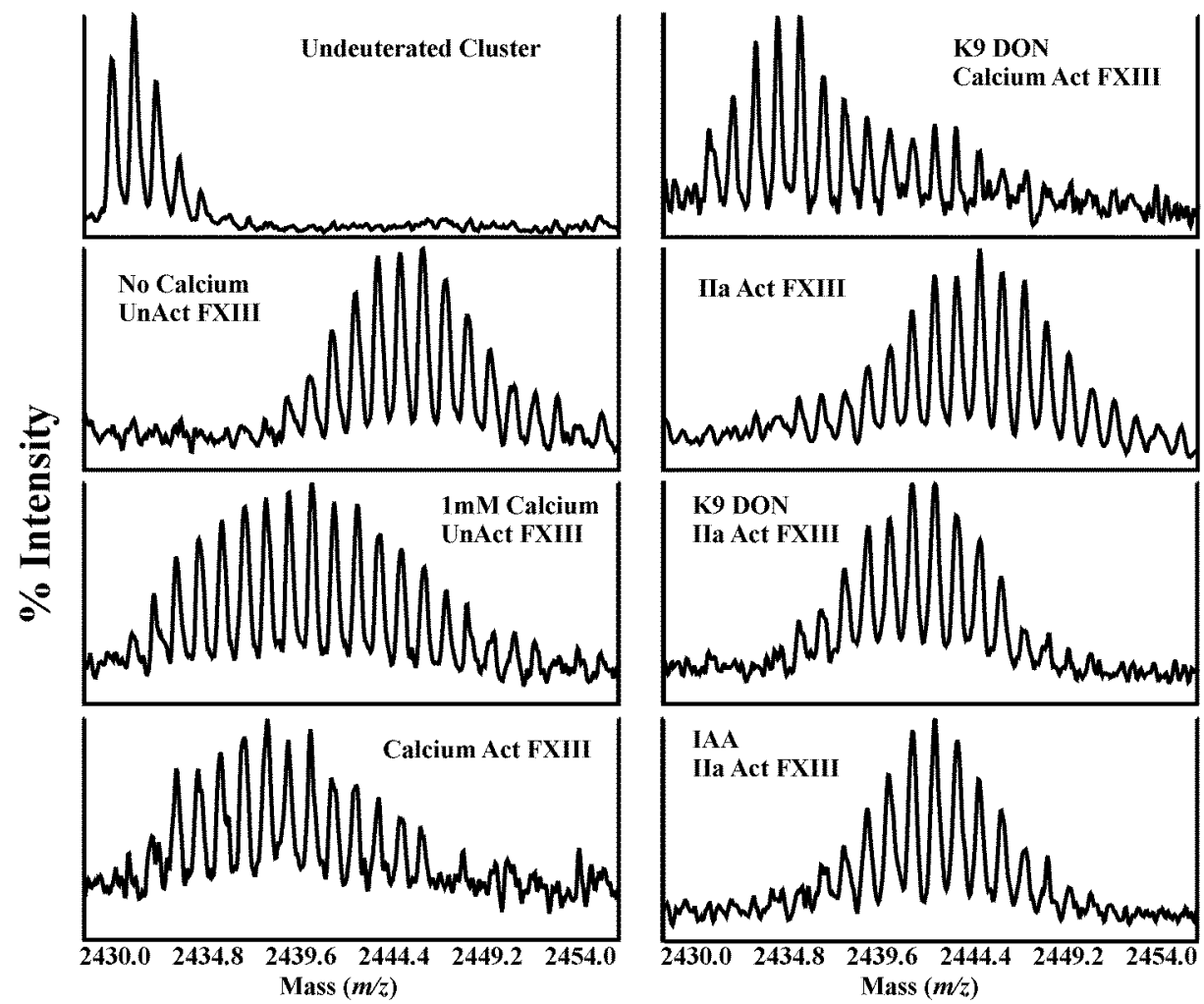

\section{Residues 526-546}

$\beta$-Barrel 1

Figure 50: Mass spectra of FXIII residues 526-546 after ten minutes of HDX. All D-on

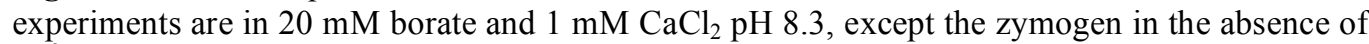
$\mathrm{Ca}^{2+}$ where the buffer was $20 \mathrm{mM}$ borate $\mathrm{pH}$ 8.3. The FXIII state illustrated in each spectrum is listed in the figure. This peak cluster contains $\beta$-barrel 1 residues and displays drastically varying degrees of deuteration between FXIIIa ${ }^{\mathrm{Ca}}$ and FXIIIa ${ }^{\mathrm{IIa}}$. In both types of activation states, inhibition provides protection from solvent amounting to around 1-2 deuterons.

N-ethylmaleimide Modification of FXIII. Digesting FXIII with the proteases trypsin and chymotrypsin provided a total of $81 \%$ sequence coverage (Figure 46). As previously reported (175), seven of the nine cysteine residues were able to be observed in the tryptic digests of FXIII: $\mathrm{C}^{152}, \mathrm{C}^{188}, \mathrm{C}^{238}, \mathrm{C}^{314}, \mathrm{C}^{327}, \mathrm{C}^{409}$, and $\mathrm{C}^{695}$. Unfortunately, chymotrypsin did not produce additional proteolytic peptides containing $\mathrm{C}^{374}$ or $\mathrm{C}^{423}$. All NEM modification experiments are summarized in Table 12.

The only consistently unmodified cysteine was $\mathrm{C}^{152}$ within the $\beta$-sandwich. For the zymogen, $\mathrm{C}^{188}$ was the lone residue to react with NEM. Once FXIII was activated, NEM 
modified the active site residue $\mathrm{C}^{314}$. Regarding the inhibited forms of FXIIIa, IAA modified $\mathrm{C}^{314}$ was observed in the mass spectra (data not shown) and a weak peak for $\mathrm{K} 9$ DON covalently linked to $\mathrm{C}^{314}$ was infrequently apparent at $2900 \mathrm{~m} / \mathrm{z}$ (Figure 45), probably due to difficulties in ionization.

\begin{tabular}{|c|c|c|c|c|c|c|c|c|c|}
\hline Cysteine Residues & $152^{a}$ & 188 & 238 & $314^{c}$ & 327 & 374 & 409 & 423 & 695 \\
\hline Zymogen & & $\mathrm{X}^{b}$ & & & & & & & \\
\hline FXIIIa $^{\mathrm{Ca}}$ & & $\mathrm{X}$ & & $\mathrm{X}$ & & & & & $X$ \\
\hline IAA FXIIIa $^{\mathrm{Ca}}$ & & $\mathrm{X}$ & & IAA & & & $\mathrm{X}$ & & $\mathrm{X}$ \\
\hline K9 DON FXIIIa ${ }^{\mathrm{Ca}}$ & & $\mathrm{X}$ & weak & K9 DON & & & & & $\mathrm{X}$ \\
\hline FXIIIa $^{\text {IIa }}$ & & & $\mathrm{X}$ & $\mathrm{X}$ & $\mathrm{X}$ & & $\mathrm{X}$ & & \\
\hline IAA FXIIIa ${ }^{\text {IIa }}$ & & & $\mathrm{X}$ & IAA & $\mathrm{X}$ & & $\mathrm{X}$ & & \\
\hline K9 DON FXIIIIa ${ }^{\text {IIa }}$ & & $\mathrm{X}$ & $\mathrm{X}$ & K9 DON & $\mathrm{X}$ & & weak & & $\mathrm{X}$ \\
\hline \multicolumn{10}{|c|}{$\begin{array}{l}{ }^{a} \text { A blank space indicates that the modified peaks were not observed in the mass spectra. } \\
{ }^{b} \text { NEM modification of cysteine was identified. }{ }^{c} \text { Cysteine } 314 \text { in the activated state reacted } \\
\text { with NEM in the uninhibited forms. The presence of the inhibitor covalently linked to } \\
\text { cysteine } 314 \text { prevented NEM from modifying the active site and the NEM peak was } \\
\text { unobserved in the mass spectra. }\end{array}$} \\
\hline
\end{tabular}

In the $\beta$-sandwich, $\mathrm{C}^{188}$ was altered by NEM in all three FXIIIa ${ }^{\mathrm{Ca}}$ states. Moving to the catalytic core, the residue $\mathrm{C}^{238}$ was weakly modified in $\mathrm{K} 9 \mathrm{DON}$ FXIIIa ${ }^{\mathrm{Ca}}$, while $\mathrm{C}^{409}$ reacted with NEM only when IAA inhibited FXIIIa ${ }^{\mathrm{Ca}} \cdot \mathrm{C}^{327}$ remained unmodified after $\mathrm{Ca}^{2+}$ activation. The $\beta$-barrel 2 residue $\mathrm{C}^{695}$ was available to NEM in all three forms of FXIIIa $^{\mathrm{Ca}}$. Turning to IIa activation, K9 DON FXIIIa ${ }^{\text {IIa }}$ was the only form of FXIIIa ${ }^{\text {IIa }}$ to display modification of the $\beta$-sandwich residue $C^{188}$ and the $\beta$-barrel 2 residue $C^{695}$. Two cysteine residues located near the activation peptide, 238 and 327, were consistently labeled in all forms of FXIIIa ${ }^{\text {IIa }}$. Finally, the catalytic core $\mathrm{C}^{409}$ was modified in FXIIIa ${ }^{\text {IIa }}$ and IAA FXIIIa ${ }^{\text {IIa }}$, but K9 DON FXIIIa ${ }^{\text {IIa }}$ severely weakened chemical modification. 
Acetic Anhydride Modification of FXIII. There are 38 lysine residues in FXIII spanning all four domains. Only 10 of the 38 lysine residues were unable to be observed as acetylated in the mass spectra: $\mathrm{K}^{61}, \mathrm{~K}^{269}, \mathrm{~K}^{363}, \mathrm{~K}^{366}, \mathrm{~K}^{418}, \mathrm{~K}^{445}, \mathrm{~K}^{446}, \mathrm{~K}^{462}, \mathrm{~K}^{467}$, and $\mathrm{K}^{657}$. Residues always accessible to acetic anhydride included $\mathrm{K}^{54}, \mathrm{~K}^{133}, \mathrm{~K}^{534}, \mathrm{~K}^{623}, \mathrm{~K}^{635}$, and $\mathrm{K}^{704}$. Additional labeling of the zymogen involved $\mathrm{K}^{129}, \mathrm{~K}^{583}, \mathrm{~K}^{584}$, and $\mathrm{K}^{621}$. Lysine residues $\mathrm{K}^{73}, \mathrm{~K}^{113}, \mathrm{~K}^{156}, \mathrm{~K}^{199}, \mathrm{~K}^{221}, \mathrm{~K}^{257}$, and $\mathrm{K}^{531}$, were exposed to acetic anhydride only after activation of FXIII. The modifications recorded from both trypsin and chymotrypsin digests will be combined and the data separated into tables concerning FXIIIa $^{\mathrm{Ca}}$ (Table 13) and FXIIIa ${ }^{\text {IIa }}$ (Table 14).

\begin{tabular}{|c|c|c|c|c|c|c|c|c|c|c|c|c|c|c|c|}
\hline \multirow[b]{2}{*}{ Residues } & \multicolumn{8}{|c|}{$\beta$ sandwich } & \multicolumn{7}{|c|}{ Catalytic Core } \\
\hline & $54^{a}$ & 68 & 73 & 113 & 129 & 133 & 151 & 156 & 199 & 221 & 257 & 482 & 503 & 504 & 513 \\
\hline Zymogen & $X^{b}$ & & & $?^{c}$ & $X$ & $\mathrm{X}$ & & & & & & & & $?$ & $?$ \\
\hline FXIIIa $^{\mathrm{Ca}}$ & $\mathrm{X}$ & & $\mathrm{X}$ & $\mathrm{X}$ & & $\mathrm{X}$ & $?$ & $X$ & $\mathrm{X}$ & $\mathrm{X}$ & $\mathrm{X}$ & $\mathrm{X}$ & ? & $?$ & $?$ \\
\hline $\begin{array}{l}\text { IAA } \\
\text { FXIIIa }^{\mathrm{Ca}}\end{array}$ & $\mathrm{X}$ & & $\mathrm{X}$ & $\mathrm{X}$ & & $\mathrm{X}$ & ? & $\mathrm{X}$ & $\mathrm{X}$ & $\mathrm{X}$ & $\mathrm{X}$ & $\mathrm{X}$ & $?$ & $?$ & \\
\hline $\begin{array}{l}\text { K9 DON } \\
\text { FXIIIa }^{\text {Ca }}\end{array}$ & $\mathrm{X}$ & & $\mathrm{X}$ & $X$ & & $\mathrm{X}$ & $?$ & X & $\mathrm{X}$ & $X$ & $\mathrm{X}$ & & & $?$ & $?$ \\
\hline & \multicolumn{8}{|c|}{$\beta$ barrel 1} & \multicolumn{5}{|c|}{$\beta$ barrel 2} & & \\
\hline Residues & 531 & 534 & 565 & 569 & 570 & 583 & 584 & 621 & 623 & 635 & 677 & 678 & 704 & & \\
\hline Zymogen & $?$ & $\mathrm{X}$ & & & & $\mathrm{X}$ & $X$ & $\mathrm{X}$ & X & $X$ & $?$ & $?$ & $\mathrm{X}$ & & \\
\hline FXIIIa $^{\mathrm{Ca}}$ & $\mathrm{X}$ & $\mathrm{X}$ & $\mathrm{X}$ & & $\mathrm{X}$ & $\mathrm{X}$ & $\mathrm{X}$ & $\mathrm{X}$ & $\mathrm{X}$ & $\mathrm{X}$ & $?$ & $?$ & $\mathrm{X}$ & & \\
\hline $\begin{array}{c}\text { IAA } \\
\text { FXIIIa }^{\mathrm{Ca}}\end{array}$ & $\mathrm{X}$ & $\mathrm{X}$ & $?$ & $?$ & $\mathrm{X}$ & $\mathrm{X}$ & $\mathrm{X}$ & $\mathrm{X}$ & $\mathrm{X}$ & $\mathrm{X}$ & $?$ & $?$ & $\mathrm{X}$ & & \\
\hline $\begin{array}{l}\text { K9 DON } \\
\text { FXIIIa }^{\mathrm{Ca}}\end{array}$ & $\mathrm{X}$ & $\mathrm{X}$ & $X$ & $?$ & $?$ & $\mathrm{X}$ & X & $\mathrm{X}$ & $\mathrm{X}$ & $\mathrm{X}$ & ? & ? & $\mathrm{X}$ & & \\
\hline
\end{tabular}

${ }^{a}$ Residues displaying modification regardless of FXIII activation or inhibition are referred to as always solvent exposed. ${ }^{b}$ The letter $\mathrm{X}$ represents lysine modification of the indicated residue. ${ }^{c} \mathrm{~A}$ question mark denotes ambiguous data and the possibility exists for modification of the lysine residue. The first possibility is when two peptides have similar masses and both possess lysine residues. Alternatively, a peptide with two or three lysine residues may be observed as acetylated once, thus presenting ambiguity in assigning the modified lysine.

In the $\beta$ sandwich, $\mathrm{K}^{68}$ and $\mathrm{K}^{129}$ were not accessible to acetic anhydride modification in all forms of FXIIIa ${ }^{\mathrm{Ca}}$. Interestingly, the catalytic core $\mathrm{K}^{482}$ was labeled in FXIIIa $^{\mathrm{Ca}}$ and IAA FXIIIa ${ }^{\mathrm{Ca}}$, yet unmodified in K9 DON FXIIIa ${ }^{\mathrm{Ca}}$. For all three states of 
FXIIIa ${ }^{\mathrm{Ca}}$, the $\beta$ barrel 1 residues $\mathrm{K}^{583}, \mathrm{~K}^{584}$ and $\mathrm{K}^{621}$ were modified, while both $\mathrm{K}^{565}$ and $\mathrm{K}^{570}$ could not be unambiguously assigned as always labeled. Acetylation results revealed that six residues spanning the $\beta$ sandwich and catalytic core displayed consistent labeling in FXIIIa ${ }^{\text {IIa }}$ : $\mathrm{K}^{68}, \mathrm{~K}^{129}, \mathrm{~K}^{482}, \mathrm{~K}^{503}, \mathrm{~K}^{504}$, and $\mathrm{K}^{513}$ (Table 14). Like FXIIIa ${ }^{\mathrm{Ca}}$, $\mathrm{K}^{565}$, could not be unambiguously assigned. $\mathrm{K}^{583}$ and $\mathrm{K}^{584}$ did not react with acetic anhydride when IIa activated FXIII, however, general inhibition of FXIIIa ${ }^{\text {IIa }}$ resulted in protection from acetic anhydride for the $\beta$ barrel 1 residue $\mathrm{K}^{621}$.

\begin{tabular}{|c|c|c|c|c|c|c|c|c|c|c|c|c|c|c|c|}
\hline \multirow[b]{2}{*}{ Residues } & \multicolumn{8}{|c|}{$\beta$ sandwich } & \multicolumn{7}{|c|}{ Catalytic Core } \\
\hline & $54^{a}$ & 68 & 73 & 113 & 129 & 133 & 151 & 156 & 199 & 221 & 257 & 482 & 503 & 504 & 513 \\
\hline Zymogen & $\mathrm{X}^{b}$ & & & $?^{c}$ & $\mathrm{X}$ & $\mathrm{X}$ & & & & & & & & $?$ & $?$ \\
\hline FXIIIIa ${ }^{\text {IIa }}$ & $\mathrm{X}$ & $\mathrm{X}$ & $\mathrm{X}$ & $\mathrm{X}$ & $\mathrm{X}$ & $\mathrm{X}$ & $?$ & $\mathrm{X}$ & $\mathrm{X}$ & $\mathrm{X}$ & $\mathrm{X}$ & $\mathrm{X}$ & $\mathrm{X}$ & $\mathrm{X}$ & $\mathrm{X}$ \\
\hline $\begin{array}{c}\text { IAA } \\
\text { FXIIIIa }^{\text {IIa }}\end{array}$ & $\mathrm{X}$ & $\mathrm{X}$ & $\mathrm{X}$ & $\mathrm{X}$ & $\mathrm{X}$ & $\mathrm{X}$ & $?$ & $\mathrm{X}$ & X & $\mathrm{X}$ & $\mathrm{X}$ & $\mathrm{X}$ & $\mathrm{X}$ & $\mathrm{X}$ & $\mathrm{X}$ \\
\hline $\begin{array}{l}\text { K9 DON } \\
\text { FXIIIIa }^{\text {IIa }}\end{array}$ & X & $\mathrm{X}$ & $\mathrm{X}$ & $\mathrm{X}$ & X & $\mathrm{X}$ & $?$ & $\mathrm{X}$ & X & X & X & $\mathrm{X}$ & $\mathrm{X}$ & X & $\mathrm{X}$ \\
\hline & \multicolumn{8}{|c|}{$\beta$ barrel 1} & \multicolumn{5}{|c|}{$\beta$ barrel 2} & & \\
\hline Residues & 531 & 534 & 565 & 569 & 570 & 583 & 584 & 621 & 623 & 635 & 677 & 678 & 704 & & \\
\hline Zymogen & $?$ & $\mathrm{X}$ & & & & $\mathrm{X}$ & $\mathrm{X}$ & $\mathrm{X}$ & $\mathrm{X}$ & $\mathrm{X}$ & $?$ & $?$ & $\mathrm{X}$ & & \\
\hline FXIIIIa ${ }^{\text {IIa }}$ & $\mathrm{X}$ & $\mathrm{X}$ & $\mathrm{X}$ & $?$ & $?$ & & & $\mathrm{X}$ & $\mathrm{X}$ & $\mathrm{X}$ & $?$ & $?$ & $\mathrm{X}$ & & \\
\hline $\begin{array}{c}\text { IAA } \\
\text { FXIIIa }^{\text {IIa }}\end{array}$ & X & X & $\mathrm{X}$ & & & & & & X & X & ? & $?$ & $\mathrm{X}$ & & \\
\hline $\begin{array}{l}\text { K9 DON } \\
\text { FXIIIa }\end{array}$ & X & X & ? & ? & ? & & & & X & $\mathrm{X}$ & ? & ? & $\mathrm{X}$ & & \\
\hline
\end{tabular}

${ }^{a}$ Residues displaying modification regardless of FXIII activation or inhibition are referred to as always solvent exposed. ${ }^{b}$ The letter $\mathrm{X}$ represents lysine modification of the indicated residue. ${ }^{c} \mathrm{~A}$ question mark denotes ambiguous data and the possibility exists for modification of the lysine residue. The first possibility is when two peptides have similar masses and both possess lysine residues. Alternatively, a peptide with two or three lysine residues may be observed as acetylated once, thus presenting ambiguity in assigning the modified lysine.

Limited Proteolysis of FXIII. Variations within the mass spectra of FXIII(a) provide insight into subtle alterations in conformational dynamics that affect chymotrypsin's ability to hydrolyze backbone amide bonds of the TGase. For example, during IIa activation of FXIII, the amide bond between $\mathrm{R}^{36}-\mathrm{G}^{37}$ is cleaved and a chymotryptic digest of FXIIIa ${ }^{\text {IIa }}$ does not produce the fragment $\mathrm{Q}^{32}-\mathrm{F}^{44}$. Proteolysis of 
FXIIIIa $^{\text {IIa }}$ by chymotrypsin produces a significant amount of the activation peptide residues $G^{9}-L^{31}$. By contrast, the digests of the zymogen and FXIIIa ${ }^{\text {Ca }}$ displayed the peptide $Q^{32}-F^{44}$ and less $G^{9}-L^{31}$. In our studies with $\mathrm{FXIII}(\mathrm{a})$, significant variation within thirty minutes of trypsin proteolysis was not observed (data not shown), thus the focus was centered on chymotryptic digests of FXIII(a). Selected data for chymotrypsin proteolysis is summarized in Figure 51. Within the $\beta$-sandwich, two peptides were cleaved more effectively with FXIIIa $^{\mathrm{Ca}}$ : $75-83$ and 104-125. FXIIIa ${ }^{\text {IIa }}$ displayed a large abundance of the catalytic core fragment 207-214. Finally, two fragments in the $\beta$ barrel 2, 681-691 and 692-698, were more susceptible to chymotrypsin in FXIIIa ${ }^{\mathrm{Ca}}$. The inhibitor K9 DON did not significantly affect the chymotryptic digests profiles.

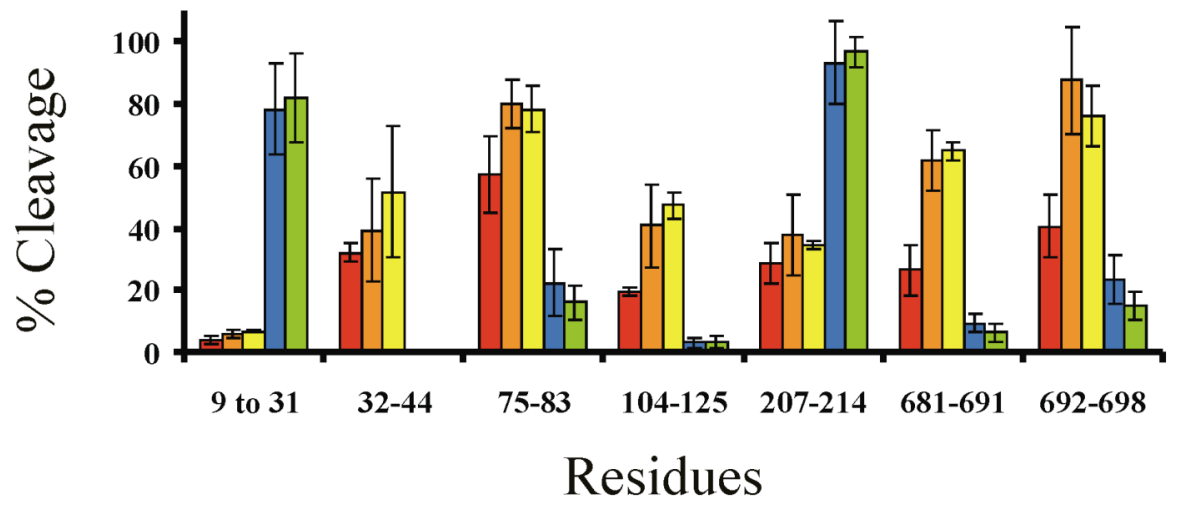

Figure 51: Graph of limited chymotryptic proteolysis of FXIII. Only differences in proteolysis larger than $\pm 20 \%$ were considered significant. The colored bars refer to the zymogen (red), FXIIIa ${ }^{\mathrm{Ca}}$ (orange), K9 DON FXIIIa ${ }^{\mathrm{Ca}}$ (yellow), FXIIIa ${ }^{\text {IIa }}$ (blue), and K9 DON FXIIIa ${ }^{\text {IIa }}$ (green). Errors correspond to standard deviation of the mean for three independent trials.

\section{Discussion}

At the present time, an X-ray structure of FXIIIa interacting with a substrate or inhibitor has not been solved. Furthermore, limited evidence exists on how Q containing substrates access the active site, interact with the catalytic core, and alter the dynamics of FXIIIa in preparation for the $\mathrm{K}$ containing substrate. An effective peptide inhibitor for 
FXIIIa has now been developed that incorporates the Q isostere 6-diazo-5-oxo-norleucine into the FXIIIa substrate peptide K9. The focus of the work has proceeded to observe in solution the conformational changes associated with inhibition by K9 DON compared to IAA (178). In addition to probing FXIIIa inhibition, new insight has also been obtained regarding the conformational features associated with the activation of FXIIIa by $\mathrm{Ca}^{2+}$ and by IIa, thus augmenting our previous investigations $(174,175)$. The current studies thus provide valuable information about events occurring to the $\beta$ sandwich, the activation peptide, the dimer interface, the catalytic core, and $\beta$ barrels 1 and 2 (178).

DON Inhibition of FXIIII ${ }^{I I a}$. A modified version of the Dade-Behring Berichrom assay (177) was used to determine that K9 DON is a slightly less potent inhibitor of FXIIIa ${ }^{\text {IIa }}$ as compared to IAA. A longer peptide derived from the natural substrate $\alpha_{2}$ antiplasmin has been reported to be an even better FXIIIa substrate model. $\alpha_{2} \mathrm{AP}(1-15)$ shows improved kinetic parameters, maintains a preference for $\mathrm{Q}^{2}$ over $\mathrm{Q}^{4}$, and suggests the existence of additional substrate specificity determinants within the active site (137). Incorporation of DON into such a longer peptide sequence could later offer more potent inhibition of FXIIIa as well as contribute to further understanding FXIIIa-ligand interactions. The research with K9 DON provides our first successful application of such a peptide inhibitor to probe changes occurring to FXIIIa conformation in solution.

The $\beta$ sandwich and Proteolytic Versus Non-proteolytic Activation. The $\beta$ sandwich residues 72-97, also known as peptide 4, are hypothesized to be involved in Q substrate recognition (Figure 52A) since synthetic peptides derived from this sequence compete for Q substrate binding to FXIIIa (145). An earlier report demonstrated that $\mathrm{K}^{73}$ was acetylated in FXIIIa ${ }^{\mathrm{Ca}}$ only (175). The present results confirm this finding as well as 
showing that FXIIIa ${ }^{\text {IIa }}$ is also modified at this position. The current research further indicates that $\mathrm{FXIIIa}{ }^{\mathrm{Ca}}$ exhibits increased chymotryptic proteolysis of residues $75-83$ within this peptide 4 region. Together these results suggest increased exposure of 73-83 upon activation, especially for FXIIIa ${ }^{\mathrm{Ca}}$. These events could signal FXIIIa preparation for Q substrate interaction with the $\beta$ sandwich (145).

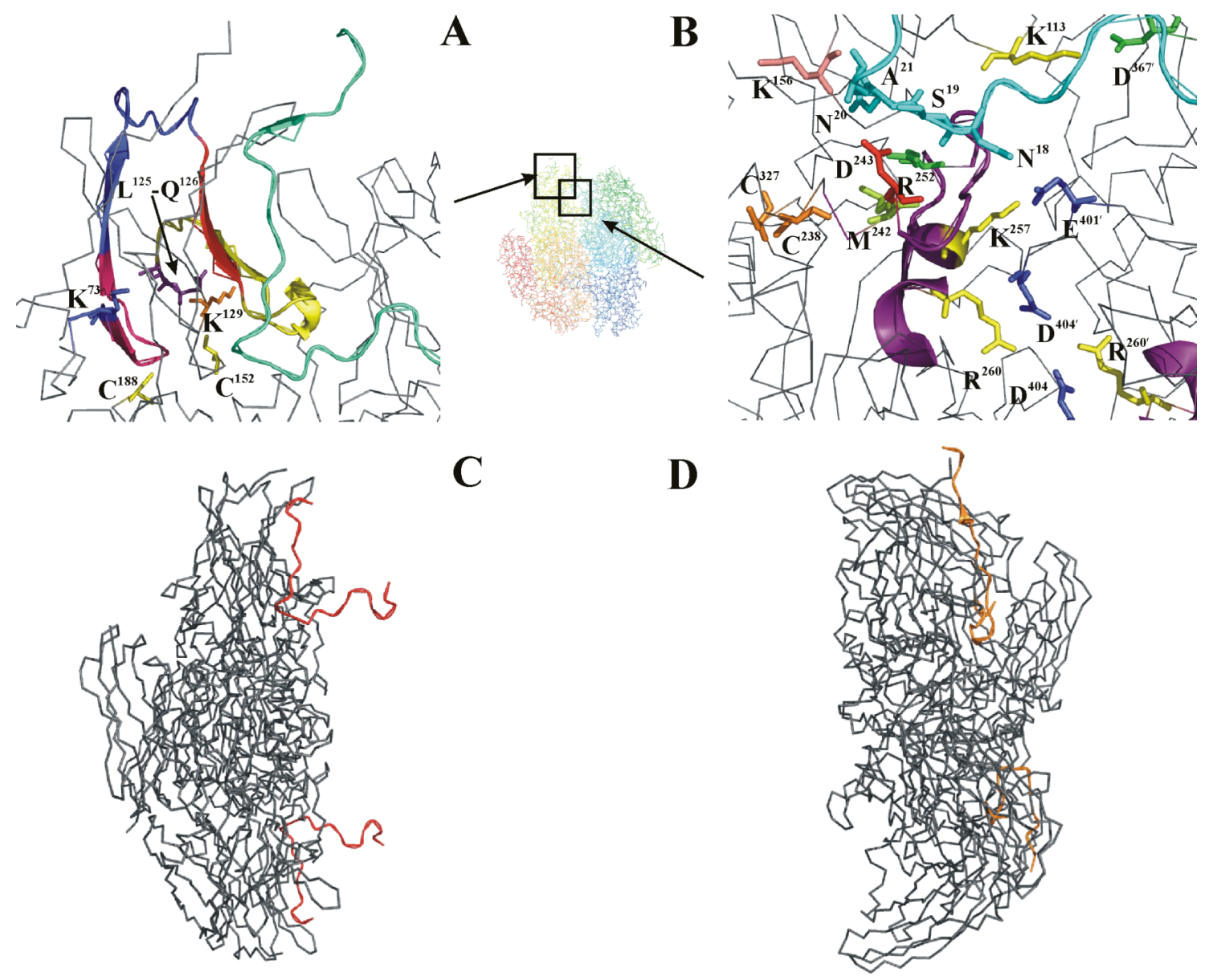

Figure 52: Correlating results with X-ray structure of Factor XIII (I) (1FIE). A. $\quad \beta$ sandwich region: Depicted are the activation peptide (cyan), peptide 4 fragments $72-74 / 84-97$ and $\mathrm{K}^{73}$ (blue), limited proteolysis fragments $75-83$ (pink) and 104-125 (yellow), $\mathrm{L}^{125}-\mathrm{Q}^{126}$ (purple), $\mathrm{K}^{129}$ (orange), the HDX fragment 98-104 (red), and $\mathrm{C}^{152} / \mathrm{C}^{188}$ (yellow). B. Activation peptide/dimer interface: residues $\mathrm{N}^{18} / \mathrm{S}^{19} / \mathrm{N}^{20} / \mathrm{A}^{21}$ (cyan), $\mathrm{K}^{156}$ (pink), $\mathrm{D}^{243}$ (red), $\mathrm{R}^{252}$ (dark green), $\mathrm{M}^{242}$ (green), $\mathrm{C}^{238} / \mathrm{C}^{327}$ (orange), the HDX fragments 240-247 and 248-264 (purple), $\mathrm{K}^{113} / \mathrm{K}^{257} / \mathrm{R}^{260}$ (yellow), $\mathrm{D}^{367^{\prime}}$ (green), and $\mathrm{E}^{401^{\prime}} / \mathrm{D}^{404^{\prime}}$ (blue). C. Zymogen crystal structure 1GGT displaying activation peptide (red) extending away from the dimer. D. FXIIIa ${ }^{\text {IIa }}$ crystal structure 1FIE (orange) displaying activation peptide resting across the surface of the dimer. 
Other unique properties related to $\mathrm{FXIII}^{\mathrm{Ca}}$ include an increase in proteolysis of the 104-125 segment, a loss of the ability to acetylate $\mathrm{K}^{129}$, and a protection from solvent for residues 98-104. As the $\mathrm{L}^{125}-\mathrm{Q}^{126}$ peptide bond becomes more accessible to chymotrypsin, it may be at the expense of $\mathrm{K}^{129}$ solvent exposure. Between peptide 4 (7297) and the chymotryptic cleavage region (104-125) is the segment 98-104. Previous HDX studies have demonstrated that residues 98-104 of FXIIIa ${ }^{\mathrm{Ca}}$ exhibited a significant decrease in deuteration that may be localized to $\mathrm{L}^{98}$ and $\mathrm{F}^{99}$ (Figure 52A) (174). IAA inhibition of FXIIIa ${ }^{\mathrm{Ca}}$ further stabilizes this short segment from deuteration. K9 DON FXIIIa $^{\mathrm{Ca}}$ requires more time to achieve significant stabilization of this fragment conceivably due to allostery related to peptide binding within the catalytic core. Interestingly, residues 98-104 are not observed peptic digests of FXIIIa ${ }^{\text {IIa }}$.

The differences in proteolysis observed for FXIIIa ${ }^{\mathrm{Ca}}$ versus FXIIIa ${ }^{\mathrm{IIa}}$ are important to consider. With both chymotrypsin and pepsin, fewer cleavages are detected within the $\beta$ sandwich region of FXIIIa ${ }^{\text {IIa }}$. Cleavage of the activation peptide by IIa may thus protect certain regions within the $\beta$ sandwich. Alternatively, the potential presence of low affinity $\mathrm{Ca}^{2+}$ binding site(s) within this domain could aide in creating a more accessible environment for the proteases to cleave FXIIIa ${ }^{\mathrm{Ca}}$. An earlier study ascertained that IAA FXIIIa ${ }^{\text {IIa }}$ possessed a higher affinity for fibrin II than IAA FXIIIa ${ }^{\text {Ca }}(269)$. Another investigation found that FXIIIa ${ }^{\mathrm{Ca}}$ was unable to crosslink fibrin $\alpha$ chains, while retaining the ability to crosslink fibrin $\gamma$ chains (270). Perhaps several fibrin-binding sites are located in or influenced by the $\beta$ sandwich and only cleavage of the activation peptide is able to properly orient or expose residues related to fibrin $\alpha$ chain binding. 
FXIII Activation Peptide and the Dimer Interface. Located in close proximity to the activation peptide (AP) and the dimer interface, FXIIIa fragments 240-247 and 248264 became more exposed to deuterium when compared to FXIII (Figure 52B). In addition, acetylation of $\mathrm{K}^{113}, \mathrm{~K}^{156}$, and $\mathrm{K}^{257}$ only occurred when FXIII was activated. Several electrostatic and water mediated interactions are apparent between the AP residues $\mathrm{N}^{18}-\mathrm{A}^{21}$ and main chain residues $\mathrm{K}^{156}, \mathrm{D}^{243}$, and $\mathrm{R}^{252}$. The crystal structures of FXIII (1GGT) (119) and FXIIIa ${ }^{\text {Ila }}$ (1FIE) (118) display the AP in similar conformations except for one key stretch. The N-terminus of the zymogen $\left(\mathrm{F}^{8}-\mathrm{P}^{16}\right)$ is extended away from the dimer, while in FXIIIa ${ }^{\text {IIa }}$ these same residues are depicted as resting on the dimer's surface (Figure 52C and D). Perhaps this emphasizes the importance of $\mathrm{N}^{18}-\mathrm{A}^{21}$ in anchoring the AP to FXIII through interactions with $\mathrm{K}^{156}, \mathrm{D}^{243}$, and $\mathrm{R}^{252}$.

Alkylation of the adjacent residues $\mathrm{C}^{238}$ and $\mathrm{C}^{327}$ occurred only with FXIIIa ${ }^{\text {Ila }}$ (Figure 52B). $\mathrm{C}^{238}$ is involved in main chain H-bonding with $\mathrm{M}^{242}$, adjacent to $\mathrm{D}^{243}$. Cleavage of the activation peptide could conceivably weaken the aforementioned electrostatic interactions involving residues within 240-264 and the activation peptide, essentially perturbing the dynamics of this region. In fact, limited proteolysis of FXIIIa ${ }^{\text {IIa }}$ shows a large abundance of the fragment 9-31. As stated in the previous section, these observations could signal FXIIIa ${ }^{\text {IIa }}$ preparation for efficient interaction with the $\alpha$ chains of fibrin. The FXIII mutations $\mathrm{M}^{242} \mathrm{~T}, \mathrm{R}^{252} \mathrm{I}$, and $\mathrm{G}^{262} \mathrm{E}$ further highlight the structural and functional importance for this stretch of catalytic core residues (271-273).

Intersubunit H-bonding among $\mathrm{K}^{113}-\mathrm{D}^{367^{\prime}}, \mathrm{K}^{257}-\mathrm{E}^{401^{\prime}}$, and $\mathrm{R}^{260}-\mathrm{D}^{404^{\prime}}$ emphasize the role these residues play in maintaining dimer integrity (Figure 7B and 52B). Acetylation of $\mathrm{K}^{113}$ and $\mathrm{K}^{257}$ and the increased exposure of 248-264 insinuate an opening of the dimer 
space. Yee et al. predict a possible mode of the $\mathrm{K}$ substrate accessing the active site through the opening of the dimer interface (119). Our results suggest activation of FXIII weakens the interactions between residues at the dimer interface. These observations are consistent with K-containing substrates accessing the active site from the dimer interface, where, as previously suggested, the acyl acceptor is directed to the active site by residue $\mathrm{D}^{343}$ and the $\mathrm{H}^{342} / \mathrm{E}^{434}$ diad (119). Three reported mutations resulting in congenital FXIII deficiency, $\mathrm{R}^{260} \mathrm{~L}, \mathrm{R}^{260} \mathrm{C}$, and $\mathrm{R}^{260} \mathrm{H}$, signify the essential nature of the $\mathrm{R}^{260}-\mathrm{D}^{404}$ interaction for proper FXIII functioning (274-277).

Cis-trans Isomerization Within the Catalytic Core and the Secondary IIa Cleavage Site. NEM modification of $\mathrm{C}^{409}$ was severely impaired by the covalent linkage of $\mathrm{K} 9$ DON to $\mathrm{C}^{314}$ in FXIIIa ${ }^{\mathrm{Ca}}$ and FXIIIa ${ }^{\text {IIa }}$ (Figure 53B). Two non-prolyl cis bonds, $\mathrm{Q}^{425}-\mathrm{F}^{426}$ and $\mathrm{R}^{310}-\mathrm{Y}^{311}$, reside near $\mathrm{C}^{314}$ and $\mathrm{C}^{409}$. These unusual cis peptide bonds may possess ample potential energy that FXIIIa could use to fuel catalysis and/or open access for substrates within the active site (278). The Ahvazi group, through molecular modeling studies on the related species tissue TGase 1 and 3 , suggest that $\mathrm{Ca}^{2+}$ binding stabilizes the non-prolyl cis bonds in preparation for catalysis and that Q substrate binding results in the cis-trans isomerization $(279,280) . \mathrm{C}^{409}$ may no longer be available for alkylation due to $\mathrm{Q}^{425}-\mathrm{F}^{426}$ isomerization, which resides about $6.5 \AA$ from $\mathrm{C}^{409}$. Another explanation could be that K9 DON interacts near or with $C^{409}$. The distance from $C^{314}$ to $C^{409}$ is $\sim 13$ $\AA$ indicating that the 5 C-terminal residues (SLVIG) of K9 DON would have to exist in an extended conformation ( $20 \AA$ in length) toward the dimer interface. This hypothesis is supported by trNOESY data that suggest the K9 peptide and an $\alpha_{2}$-antiplasmin peptide adopted extended conformations within the active site of FXIIIa ${ }^{\text {IIa }}$ (137). 

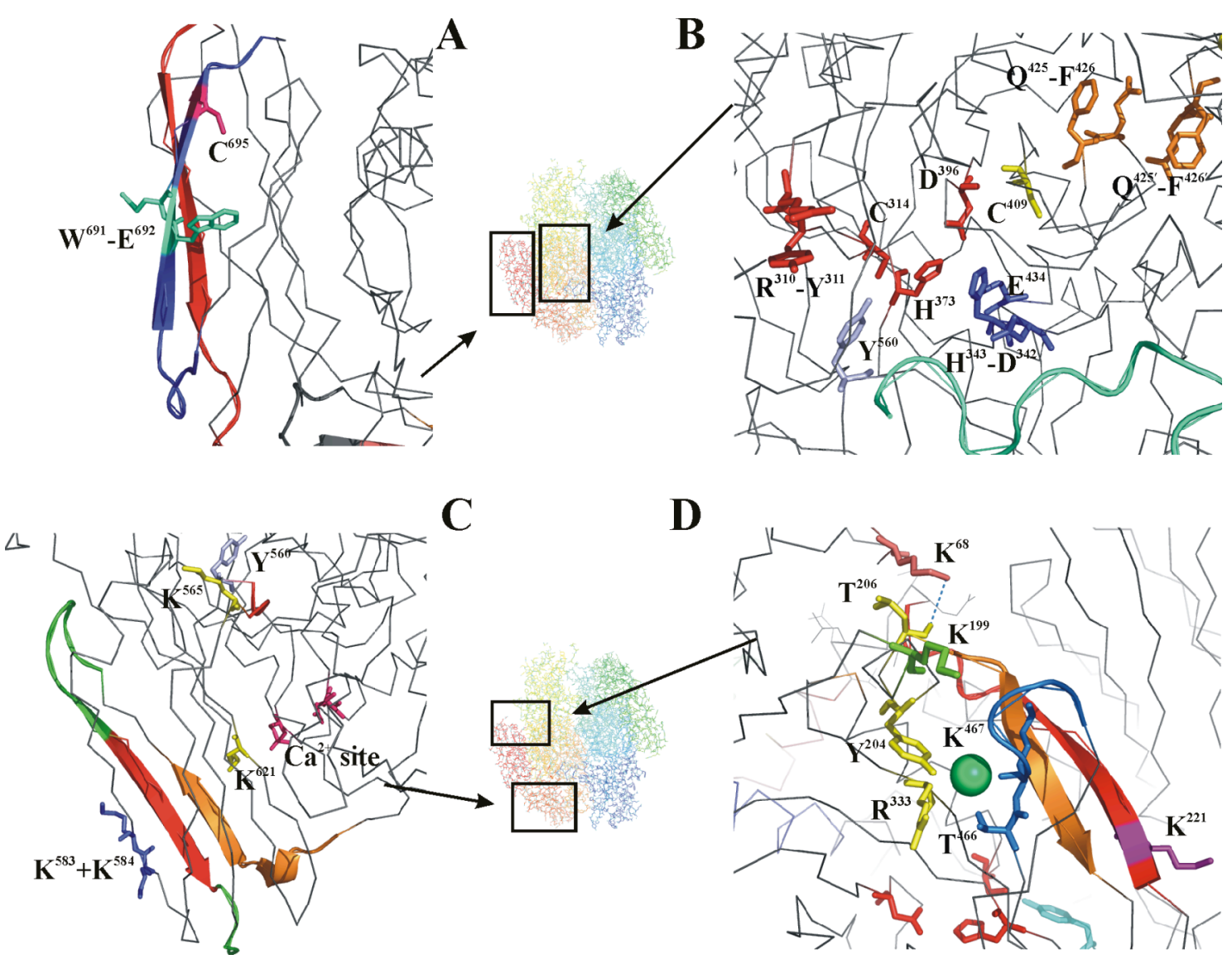

Figure 53: Correlating results with X-ray structure of Factor XIII (II) (1FIE). A. $\beta$ barrel 2: the $5 \mathrm{~A} 2$ binding epitope (red), the limited proteolysis fragment $681-698$ (blue), $\mathrm{W}^{691}-\mathrm{E}^{692}$ (cyan), and $\mathrm{C}^{695}$ (red). B. Catalytic Core I: the activation peptide (cyan), $\mathrm{D}^{342} / \mathrm{H}^{343} / \mathrm{E}^{434}$ (blue), $\mathrm{Y}^{560}$ (light blue), $\mathrm{C}^{314} / \mathrm{H}^{373} / \mathrm{D}^{396}$ (red), $\mathrm{C}^{409}$ (yellow), and the non-prolyl cis bonds $\mathrm{R}^{310}$ $\mathrm{Y}^{311}$ (red) and $\mathrm{Q}^{425}-\mathrm{F}^{426}$ (orange). C. $\beta$ barrel 1: The HDX fragments 526-534/542-546 (green), 535-541 (red), and 513-522 (orange), $\mathrm{K}^{583} / \mathrm{K}^{584}$ (blue), $\mathrm{K}^{565} / \mathrm{K}^{621}$ (yellow), $\mathrm{Y}^{560}$ (light blue), 561-564 (red), and the $\mathrm{Ca}^{2+}$ binding site (pink). D. Catalytic Core II: HDX fragment 220-230 (red), limited proteolysis fragment 207-214 (orange), and residues $\mathrm{K}^{199}$ (green), $\mathrm{K}^{221}$ (purple), $\mathrm{K}^{68}$ (red), $\mathrm{T}^{206}$ (yellow), $\mathrm{Y}^{204} / \mathrm{R}^{333}$ (yellow), and 466-472 (blue).

Another interesting observation was that $\mathrm{K}^{482}$ and $\mathrm{K}^{503}$ were not modified in $\mathrm{K} 9$ DON FXIIIa ${ }^{\mathrm{Ca}} \cdot \mathrm{K}^{482}$ is located near the $\mathrm{Ca}^{2+}$ binding site and $\mathrm{K}^{503}$ resides on a fragment leading to $\beta$ barrel 1 near the FXIIIa secondary cleavage site $\left(\mathrm{K}^{513}-\mathrm{S}^{514}\right)$. Unfortunately, fragments resulting from tryptic proteolysis of $\mathrm{K}^{513}-\mathrm{S}^{514}$ rarely appeared in the mass spectra after a thirty minute digest. Nevertheless, the acetylation results suggest that K9 DON inhibition may act in concert with $\mathrm{Ca}^{2+}$ binding to protect against further proteolysis at the secondary cleavage site by IIa. 
FXIIII ${ }^{\text {IIa }}$ : the Peptide 7 Region and Catalytic Core. The fragment 220-230 within the potential Q substrate recognition site known as peptide 7 (190-230) (145) experienced similar levels of deuterium incorporation upon inhibition, as also seen with activation. Furthermore, acetylation of both $\mathrm{K}^{221}$ and $\mathrm{K}^{199}$ only occurs in FXIIIa. Inhibition of FXIIIa did not result in hindering the modification of these residues. These findings are consistent with any of the following conclusions: residues $220-230, \mathrm{~K}^{199}$, and $\mathrm{K}^{221}$ are not involved in substrate recognition, ten residues are not long enough to reach peptide 7 , and/or Q containing substrates do not lead to allosteric effects within these regions.

Unique to FXIIIa ${ }^{\text {IIa }}$ was the increased proteolysis of the peptide 7 residues 207-214 by chymotrypsin and acetylation of $\mathrm{K}^{68}$ (Figure 53D). The distance from the $\mathrm{K}^{68}$ side chain to the backbone carbonyl of $\mathrm{L}^{206}$ is $3.8 \AA$ suggesting H-bond disruption concurrent with IIa activation. A highly conserved loop amongst all TGases exists between residues 467-472 which appear to shield a portion of peptide 7. The previous observation that $\mathrm{K}^{467}$ was acetylated upon activation (175) indicates a possible rearrangement of this loop. Curiously, Fox et al. noticed a highly conserved water at a fork between two $\beta$ strands (117), which is situated within a pocket formed by the conserved residues $\mathrm{Y}^{204}$, $\mathrm{R}^{333}, \mathrm{~T}^{466}$, and $\mathrm{K}^{467}$. The same group also observed that the strand containing $\mathrm{R}^{333}$ runs anti-parallel to the strand with $\mathrm{H}^{373}$ and that $\mathrm{T}^{466} / \mathrm{K}^{467}$ are downstream of $\mathrm{A}^{457}$ a $\mathrm{Ca}^{2+}$ binding site residue (117). This potential $\mathrm{K}^{467}$ loop repositioning could be associated with the increased exposure of peptide 7 and proper orientation of residues involved in catalysis. A study examining the consequences of TGase 1 mutations resulting in rare skin disorders commented on the significance of an $\mathrm{R}^{395} \mathrm{~L}$ ( $\mathrm{R}^{333}$ in FXIII) mutant on impairing TGase 1 activity (281). Also, the FXIII polymorphism $\mathrm{Y}^{204} \mathrm{~F}$ leads to higher 
occurrences of recurrent miscarriage, probably due to conformational changes resulting from the disruption of $\mathrm{Y}^{204}-\mathrm{R}^{333} \mathrm{H}$-bonding (282).

$\mathrm{Ca}^{2+}$ Concentration and FXIIIa Inhibition Affect Dynamics of $\beta$ barrel 1. The current HDX results confirm an earlier report of $\mathrm{Ca}^{2+}$ protecting the fragments 513-522 and 526-546 from deuterium exchange (174), however FXIIIa ${ }^{\mathrm{Ca}}$ displayed significantly more protection from solvent within this region than FXIIIa ${ }^{\text {IIa }}$. Fragment 535-541 remained at similar deuterium levels compared to the zymogen, which enabled the observed changes in dynamics to be localized to the two flanking loops, 526-534 and 542-546 (Figure 53C). The isotopic cluster for FXIII residues 526-546 exposed to $1 \mathrm{mM}$ $\mathrm{Ca}^{2+}$ appears to be an amalgam of the zymogen and FXIIIa ${ }^{\mathrm{Ca}}$ (Figure 50). This isotopic cluster possibly illustrates a concentration of $\mathrm{Ca}^{2+}$ where the cation binding site(s) is not fully occupied. Analysis of these results could be interpreted to signify allostery within the TGase. $\mathrm{Ca}^{2+}$ binding could potentially direct $\beta$ barrel 1 to "roll away" from the catalytic core to allow Q substrate access, as previously hypothesized (118).

Interestingly, the presence of K9 DON results in increased protection for 526-546 from solvent after ten minutes in both FXIIIa ${ }^{\mathrm{Ca}}$ and FXIIIa ${ }^{\text {Ila }}$. Even residues 513-522, though only in FXIIIa ${ }^{\mathrm{IIa}}$, were slightly more protected in the presence of either K9 DON or IAA. Both these inhibitors also blocked lysine acetylation of $\mathrm{K}^{621}$ in FXIIIa ${ }^{\text {IIa }}$. These results suggest that $\mathrm{Q}$ substrate binding further stabilizes $\beta$ barrel 1, perhaps restricting access to additional Q substrates in preparation for covalent crosslinking to the acyl acceptor.

Is the $\beta$ barrel 2 the Location of a Lysine Containing Substrate Recognition Site? Within $\beta$ barrel 2, chemical modification studies have provided evidence for the $\mathrm{C}^{695}$ 
exposure concurrent with activation (175). In the current work, only FXIIIa $^{\mathrm{Ca}}$ was always alkylated at $\mathrm{C}^{695}$ regardless of inhibition, while FXIIIa ${ }^{\text {IIa }}$ alkylation occurred when K9 DON was present at the active site. The discrepancy in our results is likely due to the amount of NEM used in our earlier report (200 mM versus $50 \mathrm{mM})$ (175). Further supporting this finding was the limited proteolysis studies demonstrating that FXIIIa $^{\mathrm{Ca}}$ resulted in increased chymotryptic proteolysis (chymotrypsin cleaves after W, F, and Y) of $\mathrm{W}^{691}-\mathrm{E}^{692}$, which may be related to $\mathrm{C}^{695}$ exposure (Figure 53A).

As illustrated in Mitkevich et al., the antibody 5A2 that targets the FXIIIa epitope 646-658 displays uncompetitive inhibition toward the Q substrate and competitive inhibition against the $\mathrm{K}$ substrate (146). In fact, 5A2 binding is enhanced when the acyl donor binds to the active site (146). The K9 DON FXIIIa ${ }^{\text {IIa }}$ results suggest that the Q substrate creates a more solvent accessible region within the $\beta$ barrel 2, potentially involved in K substrate recognition. This hypothesis is in line with competitive inhibition of the acyl acceptor reaction when 5A2 binds to the FXIIIa epitope 646-658 (146).

\section{$\underline{\text { Conclusions }}$}

The main objectives of this project were to demonstrate the applicability of DON based peptides in FXIIIa inhibition and to explore perturbations associated with activation and inhibition on FXIIIa dynamics. For the first time, our chemical modification and newly implemented limited proteolysis studies utilized the protease chymotrypsin in addition to trypsin. These results corroborate and further augment previous FXIII studies from our lab $(174,175)$.

New insight was obtained into the conformational variance amongst $\mathrm{FXIIIa}^{\mathrm{Ca}}$ and FXIIIa $^{\text {IIa }}$. Chymotrypsin proteolysis supplied evidence of additional peptide 7 exposure 
resulting from IIa activation: the fragment $207-214$ is cleaved more efficiently and $\mathrm{K}^{68}$ is acetylated in FXIIIa ${ }^{\text {IIa }}$. Also with FXIIII ${ }^{\text {IIa }}$, increased proteolysis of 9-31 and alkylation of the nearby residues $\mathrm{C}^{238} / \mathrm{C}^{327}$ suggest unique events associated with activation peptide cleavage. FXIIIa ${ }^{\mathrm{Ca}}$ revealed amplification in proteolysis of the peptide bond $\mathrm{L}^{125}-\mathrm{Q}^{126}$ and suppression of nearby $\mathrm{K}^{129}$ acetylation. The $\beta$ barrel 2 peptide bond $\mathrm{W}^{691}-\mathrm{E}^{692}$ and $\mathrm{C}^{695}$ in FXIIIa ${ }^{\mathrm{Ca}}$ were more solvent accessible than with FXIIIa ${ }^{\text {IIa }}$. These results perhaps indicate allostery associated with $\mathrm{Ca}^{2+}$ binding or the presence of additional $\mathrm{Ca}^{2+}$ binding sites within these domains. General activation of FXIII led to an increase in deuteration for $240-268$, as well as acetylation at $\mathrm{K}^{113}, \mathrm{~K}^{156}$, and $\mathrm{K}^{257}$. These events could indicate opening of the dimer interface for $\mathrm{K}$ substrate access to the active site.

With regard to FXIIIa inhibition, the results demonstrate that K9 DON is an effective inhibitor of FXIIIa ${ }^{\text {IIa }}$. Furthermore, the solution studies reveal potential conformational features of FXIIIa trapped in the active state by K9 DON and IAA. Specific to K9 DON inhibition was the cessation of $\mathrm{C}^{409}$ alkylation within the active site, unique perturbations in HDX for 98-104 within the $\beta$ sandwich of FXIIIa ${ }^{\mathrm{Ca}}$, increased accessibility of $\mathrm{C}^{695}$ with FXIIIa ${ }^{\mathrm{IIa}}$, and blocked $\mathrm{K}^{482} / \mathrm{K}^{503}$ acetylation in FXIIIa ${ }^{\mathrm{Ca}}$. General inhibition resulted in FXIIIa ${ }^{\text {IIa }}$ residue $\mathrm{K}^{621}$ no longer being available for acetylation and $\beta$ barrel 1 fragments 513-522 and 526-546 becoming more protected from deuteration. These observations provide subtle glimpses into how substrate bound FXIIIa prepares for the incoming lysine containing substrate. The current work focuses on the consequences of interactions at the active site of FXIIIa. Future endeavors into FXIII dynamics will examine larger more physiologically based substrates potentially evoking greater conformational changes and further targeting of putative binding exosites. 


\section{CHAPTER VII}

\section{RESEARCH SUMMARY AND FUTURE DIRECTIONS}

The primary focus of this dissertation has been ligand-protein interactions. For the research projects, two blood coagulation enzymes with very divergent catalytic activities were studied to enhance understanding of the consequences of ligand binding on protein dynamics. Chapters IV and V describe work performed on the serine protease thrombin and peptide interactions at the anion binding exosites. The peptides represent binding sites for thrombin found in fibrinogen and in the platelet receptor GpIba. Thrombin hydrolyzes peptide bonds, converting fibrinogen to fibrin and activating platelets. Another substrate for thrombin is Factor XIII. Instead of catalyzing proteolysis, the transglutaminase FXIIIa serves to crosslink adjacent proteins through the formation of a covalent bond between a glutamine and lysine residue. The studies in chapter VI illustrate the effects of an irreversible peptide inhibitor on the conformational dynamics of the transglutaminase.

Two types of instrumental approaches were employed as the primary methods for examining ligand binding to proteins in these projects: nuclear magnetic resonance (NMR) and matrix-assisted laser desorption-ionization mass spectrometry (MALDI-TOF MS). In the studies with thrombin, NMR experiments described details regarding which protons within the peptide interact with a protein and secondary structural elements the peptide assumes when bound to protein. MALDI-TOF MS proved to be an indispensable tool when investigating ligand binding from the perspective of thrombin and FXIIIa. 
Hydrogen deuterium exchange can yield information concerning sites of peptide-protein interactions. The technique can also probe the perturbations in the conformational dynamics of a protein resulting from peptide binding to a site unaffiliated with the binding interface. Two methods that provide similar information as the HDX experiments are differential chemical modification and limited proteolysis. These two techniques elucidated some of the subtle conformational transitions resulting from the activation and inhibition of FXIII.

The following sections in this chapter summarize the findings described in chapters IV, V, and VI. In addition, some preliminary work with isothermal calorimetry (ITC) has been performed to thermodynamically characterize the interactions of thrombin with the GpIb $\alpha$ peptide. Based on these results and conclusions, new avenues will be discussed for investigating ligand binding to thrombin and the mechanisms of FXIII activation and substrate recognition.

\section{$\gamma^{\prime}$ Peptide (410-427) Binding to Thrombin}

NMR and HDX coupled with MALDI-TOF MS were utilized to describe the structural and conformational features associated with ligand binding to IIa anion binding exosite-II (ABE-II) (167). In the absence of a crystal structure, the main impetus for these investigations was to gain insight into the mode and consequences of $\gamma^{\prime}$ peptide interactions at ABE-II. Many observations discussed in chapter IV correlate extremely well with the recently reported X-ray structure of the $\gamma^{\prime}$ peptide bound to thrombin (242).

The research employed 1D and 2D NMR to characterize the structural features of the bound $\gamma^{\prime}$ peptide (410-427) and to evaluate the requirement of sulfonation for effective thrombin interaction. The results indicate the $\gamma^{\prime}$ residues 414-427 make 
significant contact with thrombin; however, removing one phosphate from either tyrosine lessens the amount of observed line broadening. Interestingly, the unphosphorylated peptide does not show any $1 \mathrm{D}$ line broadening suggesting that at least one tyrosine must be phosphorylated to bind thrombin. The 2D trNOESY spectra illustrate the presence of a turn structure involving $\mathrm{Y}_{\mathrm{P}}^{422}-\mathrm{D}^{425}$ and a trans configuration for the $\mathrm{Y}_{\mathrm{P}}{ }^{422}-\mathrm{P}^{423}$ that are only evident when bound to thrombin. Once again, these structural elements require at least one phosphotyrosine. Further evidence for the turn structure at the C-terminus of the peptide is provided by the abundance of inter-residue long range NOEs, predominately centered at the aromatic rings of both phosphotyrosines.

The NMR data (167) predict many of the features depicted in the solved X-ray crystal structure (242). Only residues $413-426$ were modeled in the X-ray structure, supporting the NMR data suggesting that the first few N-terminal residues were not necessary for interacting with thrombin. Finally, the two structural elements predicted from the 2D trNOESY, the turn structure $\mathrm{Y}_{\mathrm{P}}^{422}-\mathrm{D}^{425}$ and the trans $\mathrm{Y}_{\mathrm{P}}{ }^{422}-\mathrm{P}^{423}$ bond are both present in the X-ray structure.

HDX coupled with MALDI-TOF MS was implemented to examine the location of the $\gamma^{\prime}$ peptide-thrombin interface and to screen for changes in solvent exposure at distant sites. The HDX results indicate that residues within the thrombin fragments 85-99 and 173-181 interact with the $\gamma^{\prime}$ peptide. The binding of the $\gamma^{\prime}$ peptide also protects other regions of thrombin from deuterium exchange. Affected regions include segments of ABE-I, the autolysis loop, the $\mathrm{W}^{215} / \mathrm{Na}^{+}$binding site fragment, and the A-chain. The investigation also determined that thrombin forms a ternary complex with the $\gamma^{\prime}$ peptide 
and the active site inhibitor PPACK. This interaction generates an enzyme whose solvent-exposed regions are even further stabilized from backbone amide HDX.

When comparing the data (167) to the reported crystal structure (242), the ABE-II regions 85-99 and 173-181 that were predicted to engage with $\gamma^{\prime}$ peptide contain two residues $\left(\mathrm{R}^{93}\right.$ and $\left.\mathrm{N}^{179}\right)$ that appear to bind the peptide. These observed interactions in the X-ray structure serve to further expand the interpretations of the HDX data. The crystal structure depicts electrostatic interactions involving $\mathrm{R}^{93 \mathrm{~T}}-\mathrm{S}^{420 \gamma^{\prime}}$ and $\mathrm{N}^{179 \mathrm{~T}}-\mathrm{D}^{419 \gamma^{\prime}}$. Furthermore, the $\gamma^{\prime}$ peptide appears to dimerize thrombin with many of the described secondary features observed in the NMR data forming as a result of this dimerization. In the X-ray structure, $\mathrm{R}^{93}$ of a symmetry related thrombin molecule interacts with two residues in the $\gamma^{\prime}$ peptide: $\mathrm{D}^{425}$ and $\mathrm{D}^{426}$. The $\mathrm{N}$-terminus of the $\gamma^{\prime}$ peptide fits into a hydrophobic pocket formed by several thrombin residues, one of which is $\mathrm{F}^{181}$ and several neighboring residues of the HDX protected fragment 173-181.

Another aspect of peptide interactions with thrombin's ABE-II revealed by the HDX studies concerns allostery connected to ligand binding. Quite a few residues that participate in substrate binding are contained in the coverage from a peptic digest of thrombin. In particular, residues $\mathrm{L}^{99}, \mathrm{I}^{174}$, and $\mathrm{W}^{215}$ are present in segments that are significantly protected from deuterium incorporation after one minute. These results support a connection from ABE-II to the active site that has been previously described from kinetic studies (113). The reduced rate of HDX for residues at ABE-I advocates the presence of an interexosite linkage. Similar conclusions were reached in HDX studies with ABE-I ligand binding $(172,173)$ and fluorescent work $(70,170)$. 


\section{GpIba Peptide (269-286) Binding to Thrombin}

Two crystal structures have been published illustrating the GpIbo-IIa interaction (96, 97). Whereas the $\gamma^{\prime}$ peptide-IIa interface has been localized to ABE-II, the binding site for GpIb $\alpha$ is more ambiguous and may be at ABE-II or ABE-I. The C-terminal tail of GpIb $\alpha$ (269-286) shares similar sequence elements with the $\gamma^{\prime}$ peptide. Both GpIb $\alpha$ X-ray structures $(96,97)$ display comparable ABE-II interactions for the C-terminal tail; however, the Celikel structure (1OOK) shows the C-terminal tail dimerizing thrombin through binding with ABE-I in a symmetry related thrombin molecule (97). Curiously, the $\gamma^{\prime}$ peptide-IIa structure (242) also illustrates ligand-induced dimerization of thrombin, but in this instance the $\gamma^{\prime}$ peptide binds to both thrombin molecules through ABE-II interactions.

The research performed in chapter $\mathrm{V}$ further characterizes the features of peptide binding to thrombin, building upon similar work in chapter IV on fibrin(ogen)'s $\gamma^{\prime}$ peptide. Peptides were synthesized based on the C-terminal GpIb $\alpha$ residues 269-286. Utilizing the same techniques as in chapter IV, perhaps the elements that distinguish $\gamma^{\prime}$ peptide versus GpIba peptide binding to thrombin can be discerned in more detail. Furthermore, an additional study employed analytical ultracentrifugation to determine the behavior of thrombin in the presence of both the GpIb $\alpha$ and $\gamma^{\prime}$ peptides.

1D line broadening and 2D trNOESY NMR spectra indicate that GpIbo (269-286) $\mathrm{Y}_{\mathrm{P}}{ }^{276} \mathrm{Y}_{\mathrm{P}}{ }^{278} \mathrm{Y}_{\mathrm{P}}{ }^{279}$ binds too tightly to thrombin for structural information to be obtained from these techniques. Removing phosphorylation from $\mathrm{Y}^{278}$ weakens the interaction to a certain extent and line broadening begins to be observed for the GpIb $\alpha \mathrm{Y}_{\mathrm{P}}{ }^{276} / \mathrm{Y}_{\mathrm{P}}{ }^{279}$ peptide. The largest degree of line broadening and of NOEs in the 2D trNOESY appears 
with the unphosphorylated GpIb $\alpha$ peptide. The NMR results indicate that the N-terminal residues 269-273 and the C-terminal residues 284-286 are not essential for binding thrombin. Furthermore, the GpIb $\alpha$ peptide interacts with thrombin in an extended conformation, without significant secondary structural features.

As with the $\gamma^{\prime}$ peptide, the NMR data compares very well to the X-ray structures. The residues 269-273 in the two reported GpIb $\alpha$ crystal structures are in two different conformations, which correlates to the lack of line broadening for these residues in the 1D spectra. Additionally, the extended conformation predicted by the 2D trNOESY data is also evident for the $\mathrm{C}$-terminal residues in both $\mathrm{X}$-ray structures. Interestingly, the line broadening observed for residues $\mathrm{Y}^{279}-\mathrm{D}^{283}$ and $\mathrm{E}^{285}$ may exist due to dimerization of thrombin and peptide interactions with ABE-I. The NMR data compared with the crystal structures of both GpIb $\alpha$ and the $\gamma^{\prime}$ peptide indicate that these thrombin ligands possess unique modes of binding to the serine protease.

For the HDX experiments, the GpIb $\alpha \mathrm{Y}_{\mathrm{P}}{ }^{276} \mathrm{Y}_{\mathrm{P}}{ }^{278} \mathrm{Y}_{\mathrm{P}}{ }^{279}$ peptide was used because the triply phosphorylated peptide binds tighter to thrombin. At a ratio of 20:1 peptide to enzyme, the results compare well with the data from the $\gamma^{\prime}$ peptide study, suggesting that the GpIbo binds primarily to ABE-II. More protection is observed for the ABE-I residues 65-84, perhaps stemming from ABE-I interactions with dimerized thrombin. Strikingly, after ten minutes of deuteration with the GpIba peptide, the appearance of a mixed isotopic cluster for ABE-II residues 85-99 is evident. Increasing the ratio of the GpIb $\alpha$ peptide to thrombin (40:1) during the HDX experiment leads to ABE-II fragment 85-99 experiencing a drastic reduction in protection, while ABE-I residues are now significantly protected from solvent. These results indicate that the GpIb $\alpha$ peptide is now 
binding exclusively to ABE-I. The new binding interface leads to different consequences on the dynamics of HDX for other fragments observed in the HDX experiments when compared to ABE-II binding.

The studies with analytical ultracentrifugation were performed to screen for peptide-induced dimerization of thrombin. The concentration of IIa was kept between 4.5-7 $\mu \mathrm{M}$ to avoid saturation of the uv-vis absorbance. Both the GpIb $\alpha$ peptide and the $\gamma^{\prime}$ peptide $(4.5-140 \mu \mathrm{M})$ did not exhibit dimerization of thrombin at the concentrations employed for the experiments. It should be noted that the NMR and HDX results were performed at higher concentrations of protein $(50-150 \mu \mathrm{M})$ and peptide $(1-2 \mathrm{mM})$.

These studies suggest a concentration dependent binding scheme for the GpIb $\alpha$ and IIa. This observation is not surprising given the low number of high affinity GpIb $\alpha$ binding sites on platelets $(105-1050)(101,262)$ compared to the amount GpIbo present on the surface of platelets $(25,000$ copies $)$ (263). In addition, low levels of IIa activate platelets in a GpIbo dependent manner, while high levels of IIa can perform this task without GpIb $\alpha(94,264)$. One possibility could be that GpIba binding to ABE-II serves the function of activating platelets at the initiation of blood coagulation. After the burst of IIa generation, GpIba may no longer be needed to recruit IIa to the surface of platelets to effectively interact with substrates like PAR-1. In the following sections, ongoing and future studies are described to better understand the consequences of ligand binding to ABE-II.

Preliminary Results from Isothermal Calorimetry of GpIba Binding to Thrombin

Isothermal calorimetry (ITC) was performed to thermodynamically analyze the GpIba-IIa interaction. A significant advantage of this technique rests in the ability to 
directly measure ligand-protein interactions without the need for extraneous labeling, such as fluorophores or chromophores (283). By titrating a ligand into a cell containing an interacting partner, i.e. protein, heat will be either released to the environment or absorbed from the surroundings $(283,284)$. The calorimeter measures the heat effect upon binding, which can be used to calculate the dissociation constant $\left(\mathrm{K}_{\mathrm{D}}\right)$, the enthalpy $(\Delta \mathrm{H})$ and the stoichiometry of interaction. The Gibbs free energy of an interaction determines the binding affinity: $\Delta \mathrm{G}=\mathrm{RT} \ln \mathrm{K}_{\mathrm{D}}=\Delta \mathrm{H}-\mathrm{T} \Delta \mathrm{S}$. For an optimal interaction, $\Delta \mathrm{H}$ must be negative and/or the entropy, $\Delta \mathrm{S}$, must be positive. Thus, these types of experiments can provide insight into the thermodynamic driving force for the interaction.

When analyzing the results from ITC experiments, careful consideration must be given to the interpretation of the thermodynamic parameters. Deviations in the value of $\Delta \mathrm{H}$ may not be directly correlated with changes in $\Delta \mathrm{G}$ (284). Enthalpy/entropy compensation (285) is evident in a study of glycocalicin binding to thrombin (256). Glycocalicin $(140 \mathrm{kDa})$ is the extracellular fragment of GpIba resulting from proteolysis of the receptor near the cell membrane by a protease, such as calpain $(286,287)$. By performing ITC on glycocalicin at a range of temperatures, the authors found that at lower temperatures $\left(4-10^{\circ} \mathrm{C}\right)$ the dominant driving force was entropic, while at higher temperatures $\left(20-37^{\circ} \mathrm{C}\right)$ the reverse was observed with enthalpy the major contributor to binding (256). All the while, the $\Delta \mathrm{G}$ remained relatively constant from -8.6 to -9.6 $\mathrm{kcal} / \mathrm{mol}(256)$. The authors concluded that binding was favored due to the removal of non-polar surface area from the solvent, termed the hydrophobic effect (288).

Another contribution to favorable ligand-protein interactions could be exclusively entropic in nature. In the case of thrombomodulin binding to thrombin, the interaction 
has a favorable $\Delta \mathrm{G}$ of $-11.9 \mathrm{kcal} / \mathrm{mol}$; however, ITC experiments demonstrated that $\Delta \mathrm{H}$ did not contribute to the favorable binding event (289). Thus, entropy was driving the interaction, through an increase in protein mobility and/or the release of solvent molecules from the binding interface. The authors found that ${ }^{1} \mathrm{H}-{ }^{15} \mathrm{~N}$ HSQC NMR work predicted a restriction in the configurational entropy once thrombomodulin bound to thrombin (290). The increase in entropy was primarily assigned to the displacement of water from the surface of the protein to the bulk solvent (289).

As mentioned previously, ITC has also characterized the interaction of glycocalicin and IIa (256). The authors demonstrated that the favorable binding affinity results from the burial of nonpolar surface area (256). The residues of GpIba and glycocalicin targeted by thrombin are located within the segment 269-286 (as discussed in Chapter V). In an attempt to understand the thermodynamic contribution of residues 269-286 when binding IIa, ITC was performed with the GpIb $\alpha\left(\mathrm{Y}_{\mathrm{P}}{ }^{276} \mathrm{Y}_{\mathrm{P}}{ }^{278} \mathrm{Y}_{\mathrm{P}}{ }^{279}\right)$ peptide and IIa.

The active site inhibitor PPACK was allowed to inhibit IIa for 30 min at a 2:1 ratio. An assay involving the chromogenic substrate S2238 was employed to ensure that IIa was inactive. PPACK-IIa was then subjected to dialysis in $150 \mathrm{mM} \mathrm{NaCl}, 25 \mathrm{mM}$ phosphate, $\mathrm{pH}$ 7.4. The GpIb $\alpha$ peptide was lyophilized and reconstituted in the excess buffer from dialysis. The ITC experiment attempted to characterize both the $\mathrm{K}_{\mathrm{D}}$ and $\Delta \mathrm{H}$, with the GpIb $\alpha$ peptide at $450 \mu \mathrm{M}$ and PPACK-IIa at $26.1 \mu \mathrm{M}$. PPACK-IIa was in the cell and the peptide was titrated in as $5 \mu \mathrm{L}$ aliquots every 5 minutes. The experiments were carried out at $25^{\circ} \mathrm{C}$ in a VP-ITC from Microcal. The following controls were also performed: buffer titrated into buffer, GpIb $\alpha$ peptide $(450 \mu \mathrm{M})$ titrated into buffer, and 
buffer titrated into IIa $(36.4 \mu \mathrm{M})$. The data was analyzed with the ORIGIN software supplied by MicroCal.

The data from the first ITC experiment in Figure 54A shows that the GpIb $\alpha$ $\left(\mathrm{Y}_{\mathrm{P}}^{276} \mathrm{Y}_{\mathrm{P}}^{278} \mathrm{Y}_{\mathrm{P}}{ }^{279}\right)$ peptide binding saturated thrombin too quickly to accurately observe the full sigmoidal titration curve. Thus, accurate modeling of the data by the program Origin was not possible due to the absence of the initial plateau that represents the enthalpy of binding. The experiment did indicate that GpIb $\alpha$ peptide binding to thrombin was endothermic.
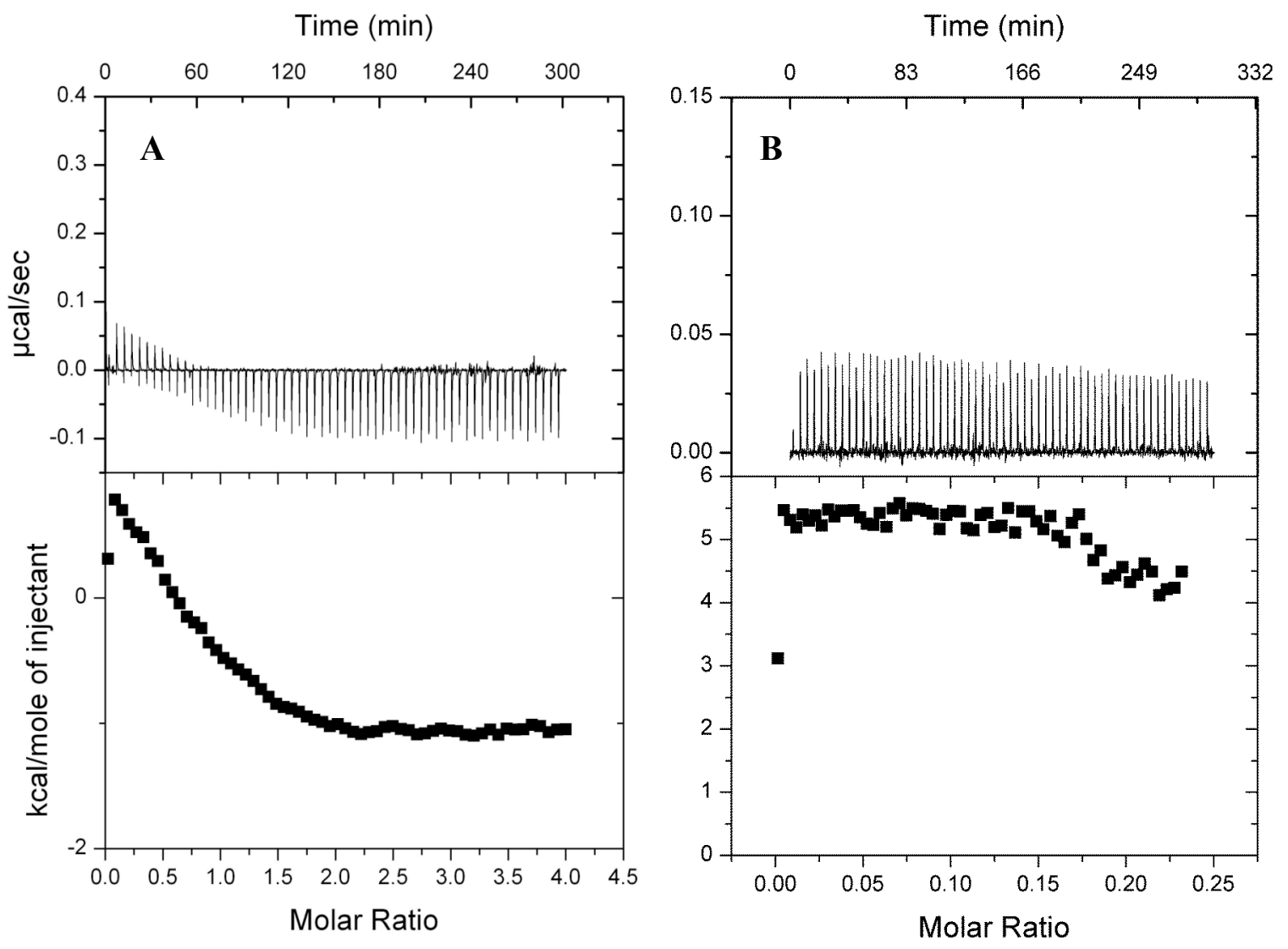

Figure 54: Isothermal calorimetry of the GpIbo peptide and thrombin. A. Top panel is the raw data showing the measured heat change of GpIb $\alpha$ peptide titrated into IIa. Bottom panel is the integrated heat of binding for GpIb $\alpha$ peptide-IIa without subtraction of the heat measured from the controls. B. Top panel is the raw data from the "model free" ITC experiment with GpIb $\alpha$ peptide titrated into thrombin. The bottom panel is the uncorrected integrated heat of binding for GpIb $\alpha$ peptide-IIa. The experiments were conducted at $298 \mathrm{~K}$ in $150 \mathrm{mM} \mathrm{NaCl}, 25 \mathrm{mM} \mathrm{H}_{3} \mathrm{PO}_{4}, \mathrm{pH}$ 7.4. 
The second type of ITC experiment was performed in order to assess the enthalpy of interaction in a "model free" manner where saturation of PPACK-IIa $(30.1 \mu \mathrm{M})$ by the GpIb $\alpha$ peptide $(30 \mu \mathrm{M})$ is not achieved (291). In order to calculate the $\Delta \mathrm{H}$, the GpIb $\alpha$ peptide was titrated into thrombin at much lower concentrations (Figure 54B), where each injection yields an independent "model-free" measurement of the $\Delta \mathrm{H}$. The results indicate that the average $\Delta \mathrm{H}$ for the interaction is about $+5.4 \mathrm{kcal} / \mathrm{mol}(291)$. The controls measuring buffer into buffer and buffer into thrombin did not display any heat evolution or absorption, discounting these possible contributors to the observed $\Delta \mathrm{H}$ (data not shown). The heat of dilution for the GpIb $\alpha$ peptide into IIa was around $-0.5 \mathrm{kcal} / \mathrm{mol}$, thus $\Delta \mathrm{H}$ for GpIbo peptide binding to IIa is closer to $+4.9 \mathrm{kcal} / \mathrm{mol}$ (data not shown).

The data from the ITC experiments indicate that the binding of the triply phosphorylated GpIba peptide is endothermic with $\Delta \mathrm{H}$ of $+4.9 \mathrm{kcal} / \mathrm{mol}$. Bothrojaracin is a thrombin inhibitor found in snake venom that binds to both ABE-I and ABE-II (292). ITC studies measuring the thermodynamics of bothrojaracin binding to ABE-I in prothrombin described a positive value for the enthalpy (13.5 kcal/mol) (293). It should be noted that ABE-II is not available in prothrombin due to binding by prothrombin fragment II (169). The report concluded that the interaction was entropically driven, but the reasons why were unclear. The authors suggested that either the release of water from a non-polar binding interface or an increase in conformational mobility of prothrombin leads to the observed positive enthalpy (293).

Both of these proposals from the bothrojaracin work seem to be unlikely explanations for the GpIbo peptide studies (293). First, the electrostatic nature of the glycocalicin interaction with thrombin was measured by Li et al. giving a $\Gamma_{\text {salt }}=-4.2(68)$. 
In addition, the GpIba peptide is highly anionic and, as demonstrated by the crystal structures, interacts with a complementary cationic binding exosite on the IIa surface (96, 97). Thus, these observations seem to indicate that the GpIb $\alpha$ peptide is not releasing water from a non-polar binding interface. Second, the HDX results at 20:1 GpIb $\alpha$ to thrombin indicate that the protein is quite protected from HDX when compared to thrombin alone (chapter V). These results suggest a restriction of the conformational ensembles sampled by IIa and a restriction of protein mobility, at least for the regions of HDX coverage.

Curiously, the $\Delta \mathrm{H}$ of glycocalicin binding to IIa at $25{ }^{\circ} \mathrm{C}$ was found to be -11.10 $\mathrm{kcal} / \mathrm{mol}$ (256). At the present time, the discrepancy between the thermodynamic behavior of glycocalicin and the GpIbo peptide is unknown. A possible explanation may be that the GpIba peptide, at the concentrations employed for ITC, dimerizes thrombin. This dimerization is observed in the Celikel crystal structure and may result in the burial of solvent accessible non-polar residues at the thrombin dimer interface (97).

Future ITC work will endeavor to further characterize the GpIb $\alpha$ peptide-IIa interaction thermodynamically. The data from Figure 54 suggests that the top and bottom of the sigmoidal binding isotherm has been observed during the course of the two experiments. By using the concentrations employed in these experiments, perhaps an experiment can be designed to generate the whole binding isotherm. If successful, these experiments could yield more detailed thermodynamic information, such as the stoichiometry of binding and the binding affinity. In addition, performing ITC experiments with the $\gamma^{\prime}$ peptide could provide important clues as to whether the positive 
enthalpies associated with the GpIb $\alpha$ peptide are a general feature of ABE-II association and possible dimerization of thrombin.

\section{Future Directions for the Study of Ligand Binding to Thrombin}

As with any endeavor, important discoveries and insights regarding the system under study generate more paths for investigation. In this section, new NMR, HDX, and AUC experiments will be suggested to help further elucidate the effects of ligand binding to thrombin. More than likely, these potential studies will lead to even more questions to be addressed in the future.

An unresolved issue from chapters IV and V (and further probed in the new ITC data of this chapter) has been whether the GpIb $\alpha$ or $\gamma^{\prime}$ peptides dimerize thrombin. The crystal structures of these ligands bound to IIa display dimerization through a symmetry related thrombin molecule. In addition, the NMR data appear to require thrombin dimerization in order to explain the observed effects. Unfortunately, peptide induced dimerization has not been independently confirmed by another solution-based method.

An NMR technique was developed in 1965 to measure the diffusion constants, termed NMR pulsed field-gradient (PFG) spin-echo technique (294). Briefly, as discussed by Stejskal and Tanner (294), during a 1D NMR spin-echo experiment, two additional gradient pulses are applied that surround the $180^{\circ}$ spin echo pulse. The first pulse dephases the transverse magnetization along the z-axis and the second pulse rephases the magnetization. While the nuclear spins are dephased, the molecules will diffuse along the z-axis. The speed of diffusion will determine the degree of attenuation required to refocus the resonances once the second pulse rephases the magnetization. 
Translational self-diffusion is related to measurable NMR parameters as described by Stejskal and Tanner (294).

The method has been used to distinguish between RNA in a duplex or RNA forming a hairpin turn and thus monomeric (295). Also, the aggregation state of the protein myosin light chain 2 has been determined employing this technique (296). By comparing the diffusion of an NMR sample of IIa alone with IIa in the presence of both peptides, the pulsed field-gradient (PFG) spin-echo technique (294) could help determine if peptide induced IIa dimerization is occurring in the NMR tube.

Another method for determining thrombin dimerization is analytical ultracentrifugation. AUC experiments were described in chapter V at significantly lower thrombin concentrations than required for NMR and crystallization. A less accurate AUC experiment could utilize the absorbance of proteins at $300-310 \mathrm{~nm}$, where saturation of the signal at $280 \mathrm{~nm}$ can be avoided when analyzing high concentrations of protein. In this case, an AUC experiment can be performed at $150 \mu \mathrm{M}$ IIa just for the express purpose of determining if two macromolecular species are dimerizing in solution.

Concerning the work with HDX, the leech derived inhibitor hirudin is known to bind to ABE-I of thrombin with a tight affinity. The 40:1 HDX results with GpIbo suggest that the peptide is binding presumably at ABE-I. By performing HDX with a peptide that targets ABE-I, perhaps the results from studies with hirudin will mirror the results with GpIb $\alpha$ at 40:1, further supporting the concept of ABE-I binding. At the same time, the allosteric consequences of ligand binding to ABE-I can also be observed and compared to work performed with fragments of the ABE-I binding protein thrombomodulin. The HDX results in chapter $\mathrm{V}$ imply that simultaneous occupation of 
both ABE-II and ABE-I by the GpIb $\alpha$ peptide is not possible. Negative allostery between the two exosites is supported by fluorescent work $(70,170)$. Thus, an HDX experiment could be designed at lower GpIb $\alpha$ peptide concentrations (i.e. 20:1) in the presence of hirudin to observe whether protection is observed at both exosites.

An additional HDX experiment would study full-length protein-thrombin interactions. The peptides GpIb $\alpha$ and $\gamma^{\prime}$ are derived from the larger parent proteins. By attaching fibrinogen with the $\gamma^{\prime}$ chain or GpIb $\alpha$ (1-290) to an agarose bead, HDX can be performed to examine the effects on thrombin dynamics resulting from protein-protein binding. An E.coli expression system has been recently developed for the $\gamma^{\prime}$ chain residues 148-427 (297). As for GpIba, residues 1-290 have been expressed in $D$. melanogaster (97) and residues 1-288 have been expressed in Chinese hamster ovary cells (96). Glycocalicin, the extracellular portion of GpIba, could also be studied by using a protease to cleave GpIb $\alpha$ at the platelet surface and then the extracellular portion could be purified $(287,298)$.

For fibrinogen, the HDX projects could examine the effects of the whole fibrinogen $\gamma^{\prime}$ homodimer $\left(\mathrm{A} \alpha \mathrm{B} \beta \gamma^{\prime}\right)_{2}$ versus the heterodimer $(\mathrm{A} \alpha)_{2}(\mathrm{~B} \beta)_{2}\left(\gamma_{\mathrm{A}} \gamma^{\prime}\right)$. Within the HDX protocol, one more step would be added to separate the thrombin from the protein attached to the agarose bead, perhaps involving an acidic quench (0.1\% TFA) in a solvent of lower polarity (i.e. propanol) to disrupt the interaction (299). This would be followed by centrifugation, then peptic digestion. Finally, the binding could be studied from the perspective of GpIb $\alpha$ (1-290), with IIa attached to the bead. Both of these proteins are of similar length. In this case, the fragments from a peptic digest of GpIb $\alpha$ would have to be identified by PSD and carboxypeptidase Y sequencing. HDX work has been 
performed with a similar leucine rich repeat protein $\mathrm{I} \kappa \mathrm{B} \alpha$, which is involved in transcriptional regulation of NF-אB (300).

\section{$\underline{\text { FXIII Activation and Inhibition Studies }}$}

Another important substrate for thrombin is the transglutaminase Factor XIII. To date, crystallography has been unable to accurately model the conformational transition that occurs once FXIII is activated by thrombin (117-119). Therefore, solution based techniques have been performed to characterize some of the subtle changes in FXIII dynamics that results from activation. MALDI-TOF MS was utilized to observe the effects of activation and inhibition on FXIII dynamics through HDX, differential chemical modification, and limited proteolysis (178).

The work described in chapter VI further builds upon research performed by Brian T. Turner, Jr. $(174,175)$ by increasing FXIII sequence coverage with the protease chymotrypsin and by binding the irreversible peptide inhibitor K9 DON to the active site. Previous HDX experiments demonstrated that FXIII activation protects regions within the $\beta$ sandwich (98-104) and the $\beta$ barrel 1 (526-546) from deuterium, while exposing the potential Q substrate recognition site (220-230) to deuteration (174). Differential chemical modification indicated the availability of several residues upon activation including $\mathrm{K}^{73}, \mathrm{~K}^{221}, \mathrm{C}^{314}$, and $\mathrm{C}^{409}$ (175). These initial forays into FXIII dynamics supported the notion that conformational changes occur after FXIII activation that are currently undetected in the crystal structures of the transglutaminase (117-119).

In the current work (178), activations of FXIII by thrombin and calcium were further examined. New findings unique to FXIIIa ${ }^{\text {IIa }}$ (thrombin activated FXIII) included additional evidence for the exposure of peptide 7 with the acetylation of $\mathrm{K}^{68}$ and 
increased proteolysis of 207-214. Peptide 7 is hypothesized to be a potential substrate recognition site accessible after FXIII activation (145). Thrombin cleavage of the activation peptide exposed resides $\mathrm{C}^{238}$ and $\mathrm{C}^{327}$ to alkylation by NEM, as well as enhanced proteolysis of 9-31. By contrast, FXIIIa $^{\mathrm{Ca}}$ (calcium activated FXIII) led to increased proteolysis of the $\beta$ sandwich fragments $75-83$ and 104-125 and a loss of $\mathrm{K}^{129}$ acetylation. In addition, several residues within the $\beta$ barrel 2 were more solvent exposed in FXIIIa ${ }^{\mathrm{Ca}}$ including the peptide bond $\mathrm{W}^{691}-\mathrm{E}^{692}$ and $\mathrm{C}^{695}$. These observations may be connected to the allosteric transmission of calcium binding at undiscovered sites proposed by two independent groups $(131,132)$. Finally, activating FXIII with either thrombin or calcium appeared to increase access within the dimer interface, as evidenced by the enhancement of deuterium incorporation for residues 240-268 and acetylation of $\mathrm{K}^{113}, \mathrm{~K}^{156}$, and $\mathrm{K}^{257}$.

The investigation also examined FXIIIa inhibition by the K9 DON peptide (with the glutamine isostere 6-diazo-5-oxo-norleucine) compared with the nonspecific alkylating agent iodoacetamide (IAA). The results illustrate, for the first time, irreversible inhibition of FXIIIa with a peptide-derived inhibitor. K9 DON prevented alkylation of $\mathrm{C}^{409}$ within the catalytic core perhaps a result of the inhibitor interacting near this residue within the active site. Other observations related to K9 DON inhibition may signal preparation for the incoming lysine containing substrate, including alkylation of $\mathrm{C}^{695}$ in FXIIIa ${ }^{\text {IIa }}$ and the cessation of $\mathrm{K}^{482}-\mathrm{K}^{503}$ acetylation in FXIIIa ${ }^{\mathrm{Ca}}$. Both inhibitors promoted even greater protection from deuteration for the $\beta$ sandwich (98-104) and the $\beta$ barrel 1 (513-522 and 526-546). The solution-based approaches reveal that activation and inhibition lead to local and long range perturbations in the conformational dynamics of 
the transglutaminase. Important glimpses are being provided on FXIIIa allostery and the presence of putative FXIIIa exosites.

Future Research for the Study of FXIII Activation and Substrate Recognition

The development of a peptide-based inhibitor of FXIIIa has created new possibilities for studying the conversion of FXIII to FXIIIa. The glutamine isostere 6diazo-5-oxo-norleucine may be incorporated into a better peptide substrate for FXIIIa $\alpha_{2}$ antiplasmin (1-15) (137). Since the reactive glutamine is at position 2 in this 15-residue peptide, inhibition of FXIIIa with $\alpha_{2}$-antiplasmin (1-15) DON will allow the C-terminal 13 residues to interact with a larger interface within the catalytic core than the four Cterminal residues of the K9 DON peptide. Similar HDX and chemical modification experiments as described in chapter VI could be performed to assess the effects of a different inhibitor on the dynamics of FXIIIa. Crystallization of DON peptide inhibited FXIIIa should also be attempted. As has been already hypothesized, the binding of an inhibitor to FXIIIa could favor crystallization of the transglutaminase in the active state, revealing aspects of the FXIIIa conformation previously unobserved.

Non-proteolytic activation of FXIII by metals is another area that lends itself to further exploration. An investigation could be conducted examining the activity of FXIII in high concentrations $(\sim 50 \mathrm{mM})$ of divalent metals such as $\mathrm{Mg}^{2+}, \mathrm{Mn}^{2+}$, and $\mathrm{Sr}^{2+} . \mathrm{A}$ question to be addressed is whether these metals can activate FXIII without the presence of calcium, in the presence of $1 \mathrm{mM}$ calcium (where the known $\mathrm{Ca}^{2+}$ binding site is occupied), or substitute for $1 \mathrm{mM} \mathrm{Ca}^{2+}$ in thrombin activated FXIII. Since the secondary reporter reactions typically used to study FXIIIa involve glutamate dehydrogenase, the presence of a variety of metals may interfere with the catalytic activity of the reporter 
enzyme. A new fluorometric assay has been recently developed to study the kinetics of the second part of the FXIII crosslinking reaction (lysine crosslinking to glutamine) (301). The reported method may be better suited to study the effects of divalent metals on FXIIIa activity since it does not rely on a secondary reporter enzyme (301).

Another aspect of FXIII that could be investigated by HDX and chemical modification is the interaction of the regulatory B-subunits of plasma FXIII with the Asubunits and with the fibrinogen $\gamma^{\prime}$ peptide. Research has shown that the B subunits of plasma FXIII binds to the $\gamma^{\prime}$ chain of fibrinogen (116). In addition, the binding interface for the B dimer has not been identified on the A dimer. The catalytic A-subunits could also be attached to agarose beads and HDX performed to study the effects of B-subunit binding to the A-subunits. As with GpIb $\alpha$ (1-290), the fragments from a peptic digest of the B subunits would have to be identified with PSD and carboxypeptidase Y sequencing.

Final Thoughts on the Investigations into Blood Coagulation Protein Interactions

The projects described in the preceding chapters have delved into further understanding the dynamics of ligand-protein interactions. The binding of two peptides with very similar sequences to thrombin leads to conformational differences in peptide structure and affects thrombin dynamics in subtly unique ways. Important insight regarding FXIIIa substrate recognition and activation has been reported with the advent of a peptide with a moiety that irreversibly inhibits FXIIIa. In the future, the specific details of these peptide interactions could perhaps be exploited to develop drugs that specifically target thrombin or Factor XIIIa in treatments for stroke, wound healing, and coronary artery disease. 


\section{REFERENCES}

1. Colman, R. W., Hirsch, J., Marder, V.J., Clowes, A.W., and George, J.N. (2001) Hemostasis and Thrombosis, Lippincott Williams and Wilkins.

2. Voet, D., and Voet, J. G. (1995) Biochemistry, John Wiley and Sons, Inc., New York, NY.

3. Schenone, M., Furie, B. C., and Furie, B. (2004) The blood coagulation cascade, Curr Opin Hematol 11, 272-277.

4. Butenas, S., and Mann, K. G. (2002) Blood coagulation, Biochemistry (Mosc) 67, 3-12.

5. Yarovaya, G. A., Blokhina, T. B., and Neshkova, E. A. (2002) Contact system. New concepts on activation mechanisms and bioregulatory functions, Biochemistry (Mosc) 67, 13-24.

6. Renne, T., and Gailani, D. (2007) Role of Factor XII in hemostasis and thrombosis: clinical implications, Expert Rev Cardiovasc Ther 5, 733-741.

7. Bolton-Maggs, P. H., and Pasi, K. J. (2003) Haemophilias A and B, Lancet 361, 1801-1809.

8. Girolami, A., Ruzzon, E., Tezza, F., Allemand, E., and Vettore, S. (2007) Congenital combined defects of factor VII: a critical review, Acta Haematol 117, 51-56.

9. Monroe, D. M., and Hoffman, M. (2006) What does it take to make the perfect clot?, Arterioscler Thromb Vasc Biol 26, 41-48.

10. Hoffman, M., and Monroe, D. M. (2007) Coagulation 2006: a modern view of hemostasis, Hematol Oncol Clin North Am 21, 1-11. 
11. Hoffman, M., and Monroe, D. M., 3rd. (2001) A cell-based model of hemostasis, Thromb Haemost 85, 958-965.

12. Nelsestuen, G. L., Shah, A. M., and Harvey, S. B. (2000) Vitamin K-dependent proteins, Vitam Horm 58, 355-389.

13. Huang, M., Rigby, A. C., Morelli, X., Grant, M. A., Huang, G., Furie, B., Seaton, B., and Furie, B. C. (2003) Structural basis of membrane binding by Gla domains of vitamin K-dependent proteins, Nat Struct Biol 10, 751-756.

14. Monroe, D. M., and Key, N. S. (2007) The tissue factor-factor VIIa complex: procoagulant activity, regulation, and multitasking, $J$ Thromb Haemost 5, 10971105 .

15. Steen, M. (2002) Factor Va-factor Xa interactions: molecular sites involved in enzyme:cofactor assembly, Scandinavian journal of clinical and laboratory investigation 237, 5-11.

16. Eigenbrot, C. (2002) Structure, function, and activation of coagulation factor VII, Current protein \& peptide science 3, 287-299.

17. Rezaie, A. R. (2006) Determinants of specificity of factor Xa interaction with its physiological inhibitors, Mini reviews in medicinal chemistry 6, 859-865.

18. Fay, P. J. (2004) Activation of factor VIII and mechanisms of cofactor action, Blood Rev 18, 1-15.

19. Ruggeri, Z. M., and Mendolicchio, G. L. (2007) Adhesion mechanisms in platelet function, Circulation research 100, 1673-1685.

20. Coughlin, S. R. (2001) Protease-activated receptors in vascular biology, Thromb Haemost 86, 298-307.

21. Fay, P. J. (2006) Factor VIII structure and function, International journal of hematology 83, 103-108.

22. Duga, S., Asselta, R., and Tenchini, M. L. (2004) Coagulation factor V, Int J Biochem Cell Biol 36, 1393-1399. 
23. Yun, T. H., Baglia, F. A., Myles, T., Navaneetham, D., Lopez, J. A., Walsh, P. N., and Leung, L. L. (2003) Thrombin activation of factor XI on activated platelets requires the interaction of factor XI and platelet glycoprotein Ib alpha with thrombin anion-binding exosites I and II, respectively, J Biol Chem 278, 4811248119.

24. Kurachi, K., Fujikawa, K., and Davie, E. W. (1980) Mechanism of activation of bovine factor XI by factor XII and factor XIIa, Biochemistry 19, 1330-1338.

25. Ahmad, S. S., Rawala, R., Cheung, W. F., Stafford, D. W., and Walsh, P. N. (1995) The role of the second growth-factor domain of human factor IXa in binding to platelets and in factor-X activation, Biochem J 310 ( Pt 2), 427-431.

26. Ahmad, S. S., Wong, M. Y., Rawala, R., Jameson, B. A., and Walsh, P. N. (1998) Coagulation factor IX residues G4-Q11 mediate its interaction with a shared factor IX/IXa binding site on activated platelets but not the assembly of the functional factor X activating complex, Biochemistry 37, 1671-1679.

27. Astermark, J., Hogg, P. J., and Stenflo, J. (1994) The gamma-carboxyglutamic acid and epidermal growth factor-like modules of factor IXa beta. Effects on the serine protease module and factor X activation, J Biol Chem 269, 3682-3689.

28. Osterud, B., Bouma, B. N., and Griffin, J. H. (1978) Human blood coagulation factor IX. Purification, properties, and mechanism of activation by activated factor XI, J Biol Chem 253, 5946-5951.

29. Sinha, D., Seaman, F. S., and Walsh, P. N. (1987) Role of calcium ions and the heavy chain of factor XIa in the activation of human coagulation factor IX, Biochemistry 26, 3768-3775.

30. Giangrande, P. (2005) Haemophilia B: Christmas disease, Expert Opin Pharmacother 6, 1517-1524.

31. Oldenburg, J., Ananyeva, N. M., and Saenko, E. L. (2004) Molecular basis of haemophilia A, Haemophilia 10 Suppl 4, 133-139.

32. Di Cera, E. (2003) Thrombin interactions, Chest 124, 11S-17S. 
33. Bode, W. (2005) The structure of thrombin, a chameleon-like proteinase, $J$ Thromb Haemost 3, 2379-2388.

34. Huntington, J. A. (2005) Molecular recognition mechanisms of thrombin, $J$ Thromb Haemost 3, 1861-1872.

35. Weisel, J. W. (2005) Fibrinogen and fibrin, Adv Protein Chem 70, 247-299.

36. Muszbek, L., Yee, V. C., and Hevessy, Z. (1999) Blood coagulation factor XIII: structure and function, Thromb Res 94, 271-305.

37. Lorand, L. (2001) Factor XIII: structure, activation, and interactions with fibrinogen and fibrin, Ann N Y Acad Sci 936, 291-311.

38. Bajzar, L. (2000) Thrombin activatable fibrinolysis inhibitor and an antifibrinolytic pathway, Arterioscler Thromb Vasc Biol 20, 2511-2518.

39. Walsh, P. N. (2003) Roles of factor XI, platelets and tissue factor-initiated blood coagulation, $J$ Thromb Haemost 1, 2081-2086.

40. Esmon, C. T. (2003) The protein C pathway, Chest 124, 26S-32S.

41. Dahlback, B., and Villoutreix, B. O. (2005) The anticoagulant protein C pathway, FEBS Lett 579, 3310-3316.

42. Roemisch, J., Gray, E., Hoffmann, J. N., and Wiedermann, C. J. (2002) Antithrombin: a new look at the actions of a serine protease inhibitor, Blood Coagul Fibrinolysis 13, 657-670.

43. Huntington, J. A. (2003) Mechanisms of glycosaminoglycan activation of the serpins in hemostasis, $J$ Thromb Haemost 1, 1535-1549.

44. Tollefsen, D. M. (2007) Heparin cofactor II modulates the response to vascular injury, Arterioscler Thromb Vasc Biol 27, 454-460.

45. Castellino, F. J., and Ploplis, V. A. (2005) Structure and function of the plasminogen/plasmin system, Thromb Haemost 93, 647-654. 
46. Coughlin, P. B. (2005) Antiplasmin: the forgotten serpin?, FEBS J 272, 48524857.

47. Gils, A., and Declerck, P. J. (2004) Plasminogen activator inhibitor-1, Curr Med Chem 11, 2323-2334.

48. Bode, W. (2006) The structure of thrombin: a janus-headed proteinase, Semin Thromb Hemost 32 Suppl 1, 16-31.

49. Davie, E. W., and Kulman, J. D. (2006) An overview of the structure and function of thrombin, Semin Thromb Hemost 32 Suppl 1, 3-15.

50. Bode, W., Mayr, I., Baumann, U., Huber, R., Stone, S. R., and Hofsteenge, J. (1989) The refined 1.9 A crystal structure of human alpha-thrombin: interaction with D-Phe-Pro-Arg chloromethylketone and significance of the Tyr-Pro-Pro-Trp insertion segment, Embo J 8, 3467-3475.

51. Bode, W., Turk, D., and Karshikov, A. (1992) The refined 1.9-A X-ray crystal structure of D-Phe-Pro-Arg chloromethylketone-inhibited human alpha-thrombin: structure analysis, overall structure, electrostatic properties, detailed active-site geometry, and structure-function relationships, Protein Sci 1, 426-471.

52. Kraut, J. (1977) Serine proteases: structure and mechanism of catalysis, Annu Rev Biochem 46, 331-358.

53. Hofsteenge, J., Braun, P. J., and Stone, S. R. (1988) Enzymatic properties of proteolytic derivatives of human alpha-thrombin, Biochemistry 27, 2144-2151.

54. Di Cera, E., Page, M. J., Bah, A., Bush-Pelc, L. A., and Garvey, L. C. (2007) Thrombin allostery, Phys Chem Chem Phys 9, 1291-1306.

55. Pineda, A. O., Carrell, C. J., Bush, L. A., Prasad, S., Caccia, S., Chen, Z. W., Mathews, F. S., and Di Cera, E. (2004) Molecular dissection of Na+ binding to thrombin, J Biol Chem 279, 31842-31853.

56. Wells, C. M., and Di Cera, E. (1992) Thrombin is a $\mathrm{Na}(+)$-activated enzyme, Biochemistry 31, 11721-11730. 
57. Ayala, Y. M., Cantwell, A. M., Rose, T., Bush, L. A., Arosio, D., and Di Cera, E. (2001) Molecular mapping of thrombin-receptor interactions, Proteins 45, 107116.

58. Di Cera, E., Dang, Q. D., and Ayala, Y. M. (1997) Molecular mechanisms of thrombin function, Cell Mol Life Sci 53, 701-730.

59. Adams, T. E., and Huntington, J. A. (2006) Thrombin-cofactor interactions: structural insights into regulatory mechanisms, Arterioscler Thromb Vasc Biol 26, 1738-1745.

60. Meh, D. A., Siebenlist, K. R., Brennan, S. O., Holyst, T., and Mosesson, M. W. (2001) The amino acid sequence in fibrin responsible for high affinity thrombin binding, Thromb Haemost 85, 470-474.

61. Wolfenstein-Todel, C., and Mosesson, M. W. (1981) Carboxy-terminal amino acid sequence of a human fibrinogen gamma-chain variant (gamma'), Biochemistry 20, 6146-6149.

62. Dong, J. F., Li, C. Q., and Lopez, J. A. (1994) Tyrosine sulfation of the glycoprotein Ib-IX complex: identification of sulfated residues and effect on ligand binding, Biochemistry 33, 13946-13953.

63. Michnick, D. A., Pittman, D. D., Wise, R. J., and Kaufman, R. J. (1994) Identification of individual tyrosine sulfation sites within factor VIII required for optimal activity and efficient thrombin cleavage, J Biol Chem 269, 20095-20102.

64. Olson, S. T., and Bjork, I. (1991) Predominant contribution of surface approximation to the mechanism of heparin acceleration of the antithrombinthrombin reaction. Elucidation from salt concentration effects, J Biol Chem 266, 6353-6364.

65. De Cristofaro, R., and De Filippis, V. (2003) Interaction of the 268-282 region of glycoprotein Ibalpha with the heparin-binding site of thrombin inhibits the enzyme activation of factor VIII, Biochem J 373, 593-601.

66. Richardson, J. L., Kroger, B., Hoeffken, W., Sadler, J. E., Pereira, P., Huber, R., Bode, W., and Fuentes-Prior, P. (2000) Crystal structure of the human alphathrombin-haemadin complex: an exosite II-binding inhibitor, Embo J 19, 56505660 . 
67. De Candia, E., Hall, S. W., Rutella, S., Landolfi, R., Andrews, R. K., and De Cristofaro, R. (2001) Binding of thrombin to glycoprotein Ib accelerates the hydrolysis of Par-1 on intact platelets, J Biol Chem 276, 4692-4698.

68. Li, C. Q., Vindigni, A., Sadler, J. E., and Wardell, M. R. (2001) Platelet glycoprotein $\mathrm{Ib}$ alpha binds to thrombin anion-binding exosite II inducing allosteric changes in the activity of thrombin, $J$ Biol Chem 276, 6161-6168.

69. Lovely, R. S., Moaddel, M., and Farrell, D. H. (2003) Fibrinogen gamma' chain binds thrombin exosite II, J Thromb Haemost 1, 124-131.

70. Pospisil, C. H., Stafford, A. R., Fredenburgh, J. C., and Weitz, J. I. (2003) Evidence that both exosites on thrombin participate in its high affinity interaction with fibrin, J Biol Chem 278, 21584-21591.

71. Bettelheim, F. R. (1954) Tyrosine-O-sulfate in a Peptide from Fibrinogen, J. Am. Chem. Soc. 76, 2838-2839.

72. Rydel, T. J., Tulinsky, A., Bode, W., and Huber, R. (1991) Refined structure of the hirudin-thrombin complex, J Mol Biol 221, 583-601.

73. Sheehan, J. P., and Sadler, J. E. (1994) Molecular mapping of the heparin-binding exosite of thrombin, Proc Natl Acad Sci U S A 91, 5518-5522.

74. Andrews, R. K., Gardiner, E. E., Shen, Y., Whisstock, J. C., and Berndt, M. C. (2003) Glycoprotein Ib-IX-V, Int J Biochem Cell Biol 35, 1170-1174.

75. Du, X. (2007) Signaling and regulation of the platelet glycoprotein Ib-IX-V complex, Curr Opin Hematol 14, 262-269.

76. Ozaki, Y., Asazuma, N., Suzuki-Inoue, K., and Berndt, M. C. (2005) Platelet GPIb-IX-V-dependent signaling, J Thromb Haemost 3, 1745-1751.

77. Clemetson, K. J. (2007) A short history of platelet glycoprotein Ib complex, Thromb Haemost 98, 63-68. 
78. Luo, S. Z., Mo, X., Afshar-Kharghan, V., Srinivasan, S., Lopez, J. A., and Li, R. (2007) Glycoprotein Ibalpha forms disulfide bonds with 2 glycoprotein Ibbeta subunits in the resting platelet, Blood 109, 603-609.

79. Huizinga, E. G., Tsuji, S., Romijn, R. A., Schiphorst, M. E., de Groot, P. G., Sixma, J. J., and Gros, P. (2002) Structures of glycoprotein Ibalpha and its complex with von Willebrand factor A1 domain, Science 297, 1176-1179.

80. Uff, S., Clemetson, J. M., Harrison, T., Clemetson, K. J., and Emsley, J. (2002) Crystal structure of the platelet glycoprotein Ib(alpha) N-terminal domain reveals an unmasking mechanism for receptor activation, J Biol Chem 277, 35657-35663.

81. Simon, D. I., Chen, Z., Xu, H., Li, C. Q., Dong, J., McIntire, L. V., Ballantyne, C. M., Zhang, L., Furman, M. I., Berndt, M. C., and Lopez, J. A. (2000) Platelet glycoprotein ibalpha is a counterreceptor for the leukocyte integrin Mac-1 (CD11b/CD18), J Exp Med 192, 193-204.

82. Romo, G. M., Dong, J. F., Schade, A. J., Gardiner, E. E., Kansas, G. S., Li, C. Q., McIntire, L. V., Berndt, M. C., and Lopez, J. A. (1999) The glycoprotein Ib-IX-V complex is a platelet counterreceptor for P-selectin, J Exp Med 190, 803-814.

83. Baglia, F. A., Shrimpton, C. N., Emsley, J., Kitagawa, K., Ruggeri, Z. M., Lopez, J. A., and Walsh, P. N. (2004) Factor XI interacts with the leucine-rich repeats of glycoprotein Ibalpha on the activated platelet, J Biol Chem 279, 49323-49329.

84. Bradford, H. N., Pixley, R. A., and Colman, R. W. (2000) Human factor XII binding to the glycoprotein Ib-IX-V complex inhibits thrombin-induced platelet aggregation, J Biol Chem 275, 22756-22763.

85. Bradford, H. N., Dela Cadena, R. A., Kunapuli, S. P., Dong, J. F., Lopez, J. A., and Colman, R. W. (1997) Human kininogens regulate thrombin binding to platelets through the glycoprotein Ib-IX-V complex, Blood 90, 1508-1515.

86. Chavakis, T., Santoso, S., Clemetson, K. J., Sachs, U. J., Isordia-Salas, I., Pixley, R. A., Nawroth, P. P., Colman, R. W., and Preissner, K. T. (2003) High molecular weight kininogen regulates platelet-leukocyte interactions by bridging Mac-1 and glycoprotein Ib, J Biol Chem 278, 45375-45381.

87. Ramakrishnan, V., DeGuzman, F., Bao, M., Hall, S. W., Leung, L. L., and Phillips, D. R. (2001) A thrombin receptor function for platelet glycoprotein Ib-IX 
unmasked by cleavage of glycoprotein V, Proc Natl Acad Sci U S A 98, 18231828 .

88. Ramakrishnan, V., Reeves, P. S., DeGuzman, F., Deshpande, U., MinistriMadrid, K., DuBridge, R. B., and Phillips, D. R. (1999) Increased thrombin responsiveness in platelets from mice lacking glycoprotein V, Proc Natl Acad Sci U S A 96, 13336-13341.

89. Moog, S., Mangin, P., Lenain, N., Strassel, C., Ravanat, C., Schuhler, S., Freund, M., Santer, M., Kahn, M., Nieswandt, B., Gachet, C., Cazenave, J. P., and Lanza, F. (2001) Platelet glycoprotein V binds to collagen and participates in platelet adhesion and aggregation, Blood 98, 1038-1046.

90. Vanhoorelbeke, K., Ulrichts, H., Romijn, R. A., Huizinga, E. G., and Deckmyn, H. (2004) The GPIbalpha-thrombin interaction: far from crystal clear, Trends Mol Med 10, 33-39.

91. Gralnick, H. R., Williams, S., McKeown, L. P., Hansmann, K., Fenton, J. W., 2nd, and Krutzsch, H. (1994) High-affinity alpha-thrombin binding to platelet glycoprotein Ib alpha: identification of two binding domains, Proc Natl Acad Sci U S A 91, 6334-6338.

92. De Cristofaro, R., De Candia, E., Landolfi, R., Rutella, S., and Hall, S. W. (2001) Structural and functional mapping of the thrombin domain involved in the binding to the platelet glycoprotein Ib, Biochemistry 40, 13268-13273.

93. Bouton, M. C., Thurieau, C., Guillin, M. C., and Jandrot-Perrus, M. (1998) Characteristics of the interaction between thrombin exosite 1 and the sequence 269-287 [correction of 269-297] of platelet glycoprotein Ibalpha, Thromb Haemost 80, 310-315.

94. Jandrot-Perrus, M., Bouton, M. C., Lanza, F., and Guillin, M. C. (1996) Thrombin interaction with platelet membrane glycoprotein Ib, Semin Thromb Hemost 22, $151-156$.

95. Jandrot-Perrus, M., Huisse, M. G., Krstenansky, J. L., Bezeaud, A., and Guillin, M. C. (1991) Effect of the hirudin carboxy-terminal peptide 54-65 on the interaction of thrombin with platelets, Thromb Haemost 66, 300-305. 
96. Dumas, J. J., Kumar, R., Seehra, J., Somers, W. S., and Mosyak, L. (2003) Crystal structure of the GpIbalpha-thrombin complex essential for platelet aggregation, Science 301, 222-226.

97. Celikel, R., McClintock, R. A., Roberts, J. R., Mendolicchio, G. L., Ware, J., Varughese, K. I., and Ruggeri, Z. M. (2003) Modulation of alpha-thrombin function by distinct interactions with platelet glycoprotein Ibalpha, Science 301, 218-221.

98. De Cristofaro, R., De Candia, E., Rutella, S., and Weitz, J. I. (2000) The Asp(272)-Glu(282) region of platelet glycoprotein Ibalpha interacts with the heparin-binding site of alpha-thrombin and protects the enzyme from the heparincatalyzed inhibition by antithrombin III, J Biol Chem 275, 3887-3895.

99. Olson, S. T., Halvorson, H. R., and Bjork, I. (1991) Quantitative characterization of the thrombin-heparin interaction. Discrimination between specific and nonspecific binding models, J Biol Chem 266, 6342-6352.

100. Jandrot-Perrus, M., Clemetson, K. J., Huisse, M. G., and Guillin, M. C. (1992) Thrombin interaction with platelet glycoprotein Ib: effect of glycocalicin on thrombin specificity, Blood 80, 2781-2786.

101. De Marco, L., Mazzucato, M., Masotti, A., and Ruggeri, Z. M. (1994) Localization and characterization of an alpha-thrombin-binding site on platelet glycoprotein Ib alpha, J Biol Chem 269, 6478-6484.

102. Mosesson, M. W., Siebenlist, K. R., and Meh, D. A. (2001) The structure and biological features of fibrinogen and fibrin, Ann N Y Acad Sci 936, 11-30.

103. Vali, Z., and Scheraga, H. A. (1988) Localization of the binding site on fibrin for the secondary binding site of thrombin, Biochemistry 27, 1956-1963.

104. Mosesson, M. W., Finlayson, J. S., and Umfleet, R. A. (1972) Human fibrinogen heterogeneities. 3. Identification of chain variants, J Biol Chem 247, 5223-5227.

105. Wolfenstein-Todel, C., and Mosesson, M. W. (1980) Human plasma fibrinogen heterogeneity: evidence for an extended carboxyl-terminal sequence in a normal gamma chain variant (gamma'), Proc Natl Acad Sci U S A 77, 5069-5073. 
106. Kloczewiak, M., Timmons, S., Lukas, T. J., and Hawiger, J. (1984) Platelet receptor recognition site on human fibrinogen. Synthesis and structure-function relationship of peptides corresponding to the carboxy-terminal segment of the gamma chain, Biochemistry 23, 1767-1774.

107. Chung, D. W., and Davie, E. W. (1984) gamma and gamma' chains of human fibrinogen are produced by alternative mRNA processing, Biochemistry 23, 42324236.

108. Fornace, A. J., Jr., Cummings, D. E., Comeau, C. M., Kant, J. A., and Crabtree, G. R. (1984) Structure of the human gamma-fibrinogen gene. Alternate mRNA splicing near the 3' end of the gene produces gamma A and gamma B forms of gamma-fibrinogen, $J$ Biol Chem 259, 12826-12830.

109. Mosesson, M. W., and Finlayson, J. S. (1963) Biochemical and chromatographic studies of certain activities associated with human fibrinogen preparations, $J$ Clin Invest 42, 747-755.

110. Lovely, R. S., Falls, L. A., Al-Mondhiry, H. A., Chambers, C. E., Sexton, G. J., Ni, H., and Farrell, D. H. (2002) Association of gammaA/gamma' fibrinogen levels and coronary artery disease, Thromb Haemost 88, 26-31.

111. Meh, D. A., Siebenlist, K. R., and Mosesson, M. W. (1996) Identification and characterization of the thrombin binding sites on fibrin, J Biol Chem 271, 2312123125 .

112. Nimjee, S. M., Rusconi, C. P., and Sullenger, B. A. (2005) Aptamers: an emerging class of therapeutics, Annual review of medicine 56, 555-583.

113. Siebenlist, K. R., Mosesson, M. W., Hernandez, I., Bush, L. A., Di Cera, E., Shainoff, J. R., Di Orio, J. P., and Stojanovic, L. (2005) Studies on the basis for the properties of fibrin produced from fibrinogen-containing gamma' chains, Blood 106, 2730-2736.

114. Cooper, A. V., Standeven, K. F., and Ariens, R. A. (2003) Fibrinogen gammachain splice variant gamma' alters fibrin formation and structure, Blood 102, 535540 .

115. Mosesson, M. W. (2007) Update on antithrombin I (fibrin), Thromb Haemost 98, $105-108$ 
116. Siebenlist, K. R., Meh, D. A., and Mosesson, M. W. (1996) Plasma factor XIII binds specifically to fibrinogen molecules containing gamma chains, Biochemistry 35, 10448-10453.

117. Fox, B. A., Yee, V. C., Pedersen, L. C., Le Trong, I., Bishop, P. D., Stenkamp, R. E., and Teller, D. C. (1999) Identification of the calcium binding site and a novel ytterbium site in blood coagulation factor XIII by x-ray crystallography, $J$ Biol Chem 274, 4917-4923.

118. Yee, V. C., Pedersen, L. C., Bishop, P. D., Stenkamp, R. E., and Teller, D. C. (1995) Structural evidence that the activation peptide is not released upon thrombin cleavage of factor XIII, Thromb Res 78, 389-397.

119. Yee, V. C., Pedersen, L. C., Le Trong, I., Bishop, P. D., Stenkamp, R. E., and Teller, D. C. (1994) Three-dimensional structure of a transglutaminase: human blood coagulation factor XIII, Proc Natl Acad Sci U S A 91, 7296-7300.

120. Pedersen, L. C., Yee, V. C., Bishop, P. D., Le Trong, I., Teller, D. C., and Stenkamp, R. E. (1994) Transglutaminase factor XIII uses proteinase-like catalytic triad to crosslink macromolecules, Protein Sci 3, 1131-1135.

121. Lorand, L., Gray, A. J., Brown, K., Credo, R. B., Curtis, C. G., Domanik, R. A., and Stenberg, P. (1974) Dissociation of the subunit structure of fibrin stabilizing factor during activation of the zymogen, Biochem Biophys Res Commun 56, 914922.

122. Schwartz, M. L., Pizzo, S. V., Hill, R. L., and McKee, P. A. (1973) Human Factor XIII from plasma and platelets. Molecular weights, subunit structures, proteolytic activation, and cross-linking of fibrinogen and fibrin, J Biol Chem 248, 13951407.

123. Lorand, L., and Konishi, K. (1964) Activation of the Fibrin Stabilizing Factor of Plasma by Thrombin, Arch Biochem Biophys 105, 58-67.

124. Hornyak, T. J., and Shafer, J. A. (1991) Role of calcium ion in the generation of factor XIII activity, Biochemistry 30, 6175-6182.

125. Lorand, L., and Graham, R. M. (2003) Transglutaminases: crosslinking enzymes with pleiotropic functions, Nat Rev Mol Cell Biol 4, 140-156. 
126. Ritchie, H., Lawrie, L. C., Crombie, P. W., Mosesson, M. W., and Booth, N. A. (2000) Cross-linking of plasminogen activator inhibitor 2 and alpha 2-antiplasmin to fibrin(ogen), J Biol Chem 275, 24915-24920.

127. Greenberg, C. S., Enghild, J. J., Mary, A., Dobson, J. V., and Achyuthan, K. E. (1988) Isolation of a fibrin-binding fragment from blood coagulation factor XIII capable of cross-linking fibrin(ogen), Biochem J 256, 1013-1019.

128. Lai, T. S., Achyuthan, K. E., Santiago, M. A., and Greenberg, G. S. (1994) Carboxyl-terminal truncation of recombinant factor XIII A-chains. Characterization of minimum structural requirement for transglutaminase activity, J Biol Chem 269, 24596-24601.

129. Polgar, J., Hidasi, V., and Muszbek, L. (1990) Non-proteolytic activation of cellular protransglutaminase (placenta macrophage factor XIII), Biochem $J$ 267, 557-560.

130. Credo, R. B., Curtis, C. G., and Lorand, L. (1978) Ca2+-related regulatory function of fibrinogen, Proc Natl Acad Sci U S A 75, 4234-4237.

131. Lewis, B. A., Freyssinet, J. M., and Holbrook, J. J. (1978) An equilibrium study of metal ion binding to human plasma coagulation factor XIII, Biochem J 169 , 397-402.

132. Ambrus, A., Banyai, I., Weiss, M. S., Hilgenfeld, R., Keresztessy, Z., Muszbek, L., and Fesus, L. (2001) Calcium binding of transglutaminases: a 43Ca NMR study combined with surface polarity analysis, J Biomol Struct Dyn 19, 59-74.

133. Mary, A., Achyuthan, K. E., and Greenberg, C. S. (1988) The binding of divalent metal ions to platelet factor XIII modulates its proteolysis by trypsin and thrombin, Arch Biochem Biophys 261, 112-121.

134. Achyuthan, K. E., Mary, A., and Greenberg, C. S. (1989) Tb(III)-ion-bindinginduced conformational changes in platelet factor XIII, Biochem J 257, 331-338.

135. Gorman, J. J., and Folk, J. E. (1984) Structural features of glutamine substrates for transglutaminases. Role of extended interactions in the specificity of human plasma factor XIIIa and of the guinea pig liver enzyme, J Biol Chem 259, 90079010. 
136. Sugimura, Y., Hosono, M., Wada, F., Yoshimura, T., Maki, M., and Hitomi, K. (2006) Screening for the preferred substrate sequence of transglutaminase using a phage-displayed peptide library: identification of peptide substrates for TGASE 2 and Factor XIIIA, J Biol Chem 281, 17699-17706.

137. Cleary, D. B., and Maurer, M. C. (2006) Characterizing the specificity of activated Factor XIII for glutamine-containing substrate peptides, Biochim Biophys Acta 1764, 1207-1217.

138. Coussons, P. J., Kelly, S. M., Price, N. C., Johnson, C. M., Smith, B., and Sawyer, L. (1991) Selective modification by transglutaminase of a glutamine side chain in the hinge region of the histidine-388----glutamine mutant of yeast phosphoglycerate kinase, Biochem J 273(Pt 1), 73-78.

139. Groenen, P. J., Smulders, R. H., Peters, R. F., Grootjans, J. J., van den Ijssel, P. R., Bloemendal, H., and de Jong, W. W. (1994) The amine-donor substrate specificity of tissue-type transglutaminase. Influence of amino acid residues flanking the amine-donor lysine residue, Eur J Biochem 220, 795-799.

140. Grootjans, J. J., Groenen, P. J., and de Jong, W. W. (1995) Substrate requirements for transglutaminases. Influence of the amino acid residue preceding the amine donor lysine in a native protein, $J$ Biol Chem 270, 22855-22858.

141. McDonagh, J., and Fukue, H. (1996) Determinants of substrate specificity for factor XIII, Semin Thromb Hemost 22, 369-376.

142. Folk, J. E. (1983) Mechanism and basis for specificity of transglutaminasecatalyzed epsilon-(gamma-glutamyl) lysine bond formation, Advances in enzymology and related areas of molecular biology 54, 1-56.

143. Lorand, L., and Conrad, S. M. (1984) Transglutaminases, Molecular and cellular biochemistry 58, 9-35.

144. Coussons, P. J., Price, N. C., Kelly, S. M., Smith, B., and Sawyer, L. (1992) Factors that govern the specificity of transglutaminase-catalysed modification of proteins and peptides, Biochem J 282 ( Pt 3), 929-930.

145. Achyuthan, K. E., Slaughter, T. F., Santiago, M. A., Enghild, J. J., and Greenberg, C. S. (1993) Factor XIIIa-derived peptides inhibit transglutaminase activity. Localization of substrate recognition sites, J Biol Chem 268, 21284-21292. 
146. Mitkevich, O. V., Shainoff, J. R., DiBello, P. M., Yee, V. C., Teller, D. C., Smejkal, G. B., Bishop, P. D., Kolotushkina, I. S., Fickenscher, K., and Samokhin, G. P. (1998) Coagulation factor XIIIa undergoes a conformational change evoked by glutamine substrate. Studies on kinetics of inhibition and binding of XIIIA by a cross-reacting antifibrinogen antibody, J Biol Chem 273, 14387-14391.

147. Hornyak, T. J., Bishop, P. D., and Shafer, J. A. (1989) Alpha-thrombin-catalyzed activation of human platelet factor XIII: relationship between proteolysis and factor XIIIa activity, Biochemistry 28, 7326-7332.

148. Curtis, C. G., Brown, K. L., Credo, R. B., Domanik, R. A., Gray, A., Stenberg, P., and Lorand, L. (1974) Calcium-dependent unmasking of active center cysteine during activation of fibrin stabilizing factor, Biochemistry 13, 3774-3780.

149. Curtis, C. G., Stenberg, P., Chou, C. H., Gray, A., Brown, K. L., and Lorand, L. (1973) Titration and subunit localization of active center cysteine in fibrinoligase (thrombin-activated fibrin stabilizing fector), Biochem Biophys Res Commun 52, 51-56.

150. Gan, Z. R., Li, Y., Chen, Z., Lewis, S. D., and Shafer, J. A. (1994) Identification of basic amino acid residues in thrombin essential for heparin-catalyzed inactivation by antithrombin III, J Biol Chem 269, 1301-1305.

151. Tsiang, M., Jain, A. K., and Gibbs, C. S. (1997) Functional requirements for inhibition of thrombin by antithrombin III in the presence and absence of heparin, J Biol Chem 272, 12024-12029.

152. Ye, J., Rezaie, A. R., and Esmon, C. T. (1994) Glycosaminoglycan contributions to both protein $\mathrm{C}$ activation and thrombin inhibition involve a common argininerich site in thrombin that includes residues arginine 93, 97, and 101, J Biol Chem 269, 17965-17970.

153. Tollefsen, D. M., Majerus, D. W., and Blank, M. K. (1982) Heparin cofactor II. Purification and properties of a heparin-dependent inhibitor of thrombin in human plasma, J Biol Chem 257, 2162-2169.

154. Rezaie, A. R. (2007) Heparin chain-length dependence of factor Xa inhibition by antithrombin in plasma, Thromb Res 119, 481-488. 
155. Yang, L., Manithody, C., and Rezaie, A. R. (2002) Localization of the heparin binding exosite of factor IXa, J Biol Chem 277, 50756-50760.

156. Zhao, M., Abdel-Razek, T., Sun, M. F., and Gailani, D. (1998) Characterization of a heparin binding site on the heavy chain of factor XI, J Biol Chem 273, 3115331159.

157. Sanchez, J., Elgue, G., Riesenfeld, J., and Olsson, P. (1998) Studies of adsorption, activation, and inhibition of factor XII on immobilized heparin, Thromb Res 89, 41-50.

158. Sobel, M., McNeill, P. M., Carlson, P. L., Kermode, J. C., Adelman, B., Conroy, R., and Marques, D. (1991) Heparin inhibition of von Willebrand factordependent platelet function in vitro and in vivo, J Clin Invest 87, 1787-1793.

159. Young, E., Prins, M., Levine, M. N., and Hirsh, J. (1992) Heparin binding to plasma proteins, an important mechanism for heparin resistance, Thromb Haemost 67, 639-643.

160. Barzu, T., Molho, P., Tobelem, G., Petitou, M., and Caen, J. (1985) Binding and endocytosis of heparin by human endothelial cells in culture, Biochim Biophys Acta 845, 196-203.

161. Bhandari, M., Hirsh, J., Weitz, J. I., Young, E., Venner, T. J., and Shaughnessy, S. G. (1998) The effects of standard and low molecular weight heparin on bone nodule formation in vitro, Thromb Haemost 80, 413-417.

162. Greinacher, A., and Warkentin, T. E. (2006) Recognition, treatment, and prevention of heparin-induced thrombocytopenia: review and update, Thromb Res $118,165-176$.

163. Hirsh, J., and Raschke, R. (2004) Heparin and low-molecular-weight heparin: the Seventh ACCP Conference on Antithrombotic and Thrombolytic Therapy, Chest $126,188 \mathrm{~S}-203 \mathrm{~S}$.

164. Bates, S. M., and Weitz, J. I. (2006) The status of new anticoagulants, $B r J$ Haematol 134, 3-19. 
165. Warkentin, T. E. (2004) Bivalent direct thrombin inhibitors: hirudin and bivalirudin, Best practice \& research 17, 105-125.

166. Warkentin, T. E., and Koster, A. (2005) Bivalirudin: a review, Expert Opin Pharmacother 6, 1349-1371.

167. Sabo, T. M., Farrell, D. H., and Maurer, M. C. (2006) Conformational analysis of gamma' peptide (410-427) interactions with thrombin anion binding exosite II, Biochemistry 45, 7434-7445.

168. Carter, W. J., Cama, E., and Huntington, J. A. (2005) Crystal structure of thrombin bound to heparin, $J$ Biol Chem 280, 2745-2749.

169. Arni, R. K., Padmanabhan, K., Padmanabhan, K. P., Wu, T. P., and Tulinsky, A. (1994) Structure of the non-covalent complex of prothrombin kringle 2 with PPACK-thrombin, Chem Phys Lipids 67-68, 59-66.

170. Fredenburgh, J. C., Stafford, A. R., and Weitz, J. I. (1997) Evidence for allosteric linkage between exosites 1 and 2 of thrombin, J Biol Chem 272, 25493-25499.

171. Verhamme, I. M., Olson, S. T., Tollefsen, D. M., and Bock, P. E. (2002) Binding of exosite ligands to human thrombin. Re-evaluation of allosteric linkage between thrombin exosites I and II, J Biol Chem 277, 6788-6798.

172. Koeppe, J. R., Seitova, A., Mather, T., and Komives, E. A. (2005) Thrombomodulin tightens the thrombin active site loops to promote protein $\mathrm{C}$ activation, Biochemistry 44, 14784-14791.

173. Mandell, J. G., Baerga-Ortiz, A., Akashi, S., Takio, K., and Komives, E. A. (2001) Solvent accessibility of the thrombin-thrombomodulin interface, $J \mathrm{Mol}$ Biol 306, 575-589.

174. Turner, B. T., Jr., and Maurer, M. C. (2002) Evaluating the roles of thrombin and calcium in the activation of coagulation factor XIII using H/D exchange and MALDI-TOF MS, Biochemistry 41, 7947-7954.

175. Turner, B. T., Jr., Sabo, T. M., Wilding, D., and Maurer, M. C. (2004) Mapping of factor XIII solvent accessibility as a function of activation state using chemical modification methods, Biochemistry 43, 9755-9765. 
176. Hausch, F., Halttunen, T., Maki, M., and Khosla, C. (2003) Design, synthesis, and evaluation of gluten peptide analogs as selective inhibitors of human tissue transglutaminase, Chem Biol 10, 225-231.

177. Fickenscher, K., Aab, A., and Stuber, W. (1991) A photometric assay for blood coagulation factor XIII, Thromb Haemost 65, 535-540.

178. Sabo, T. M., Brasher, P. B., and Maurer, M. C. (2007) Perturbations in Factor XIII Resulting from Activation and Inhibition Examined by Solution Based Methods and Detected by MALDI-TOF MS, Biochemistry 46, 10089 - 10101.

179. Rule, S. M., and Hitchens, T. K. (2006) Fundamentals of Protein NMR Spectroscopy, Vol. 5, Springer, Dodrecht, The Netherlands.

180. Ni, F. (1994) Recent Developments in Transferred NOE Methods, Prog. NMR Spectrosc. 26, 517-606.

181. Ni, F. (1995) 2-Dimensional Transferred Nuclear-Overhauser-Effects with Incomplete Averaging of Free-Ligand and Bound-Ligand Resonances, Journal of Magnetic Resonance Series B 106, 147-155.

182. Ni, F., and Scheraga, H. A. (1994) Use of the Transferred Nuclear Overhauser Effect to Determine the Conformations of Ligands Bound to Proteins, Accounts of Chemical Research 27, 257-264.

183. Ni, F., and Zhu, Y. (1994) Accounting for ligand-protein interactions in the relaxation-matrix analysis of transferred nuclear Overhauser effects, $J$ Magn Reson B 103, 180-184.

184. Campbell, A. P., and Sykes, B. D. (1993) The two-dimensional transferred nuclear Overhauser effect: theory and practice, Annu Rev Biophys Biomol Struct $22,99-122$.

185. Ni, F., Ripoll, D. R., Martin, P. D., and Edwards, B. F. (1992) Solution structure of a platelet receptor peptide bound to bovine alpha-thrombin, Biochemistry 31, 11551-11557. 
186. Rosevear, P. R., and Mildvan, A. S. (1989) Ligand conformations and ligandenzyme interactions as studied by the nuclear Overhauser effect, Methods Enzymol 177, 333-358.

187. Isetti, G., and Maurer, M. C. (2004) Thrombin activity is unaltered by N-terminal truncation of factor XIII activation peptides, Biochemistry 43, 4150-4159.

188. Isetti, G., and Maurer, M. C. (2004) Probing thrombin's ability to accommodate a V34F substitution within the factor XIII activation peptide segment (28-41), $J$ Pept Res 63, 241-252.

189. Maurer, M. C., Peng, J. L., An, S. S., Trosset, J. Y., Henschen-Edman, A., and Scheraga, H. A. (1998) Structural examination of the influence of phosphorylation on the binding of fibrinopeptide A to bovine thrombin, Biochemistry 37, 5888-5902.

190. Trumbo, T. A., and Maurer, M. C. (2002) Thrombin hydrolysis of V29F and V34L mutants of factor XIII (28-41) reveals roles of the $\mathrm{P}(9)$ and $\mathrm{P}(4)$ positions in factor XIII activation, Biochemistry 41, 2859-2868.

191. Trumbo, T. A., and Maurer, M. C. (2003) V34I and V34A substitutions within the factor XIII activation peptide segment (28-41) affect interactions with the thrombin active site, Thromb Haemost 89, 647-653.

192. Ni, F., Konishi, Y., and Scheraga, H. A. (1990) Thrombin-bound conformation of the $\mathrm{C}$-terminal fragments of hirudin determined by transferred nuclear Overhauser effects, Biochemistry 29, 4479-4489.

193. Ni, F., Meinwald, Y. C., Vasquez, M., and Scheraga, H. A. (1989) Highresolution NMR studies of fibrinogen-like peptides in solution: structure of a thrombin-bound peptide corresponding to residues 7-16 of the A alpha chain of human fibrinogen, Biochemistry 28, 3094-3105.

194. Ni, F., Ning, Q., Jackson, C. M., and Fenton, J. W., 2nd. (1993) Thrombin exosite for fibrinogen recognition is partially accessible in prothrombin, $J$ Biol Chem 268 , 16899-16902.

195. Ni, F., Ripoll, D. R., and Purisima, E. O. (1992) Conformational stability of a thrombin-binding peptide derived from the hirudin C-terminus, Biochemistry 31, 2545-2554. 
196. Ni, F., Zhu, Y., and Scheraga, H. A. (1995) Thrombin-Bound Structures of Designed Analogs of Human Fibrinopeptide-a Determined by Quantitative Transferred Noe Spectroscopy - a New Structural Basis for Thrombin Specificity, Journal of Molecular Biology 252, 656-671.

197. Tolkatchev, D., Ng, A., Zhu, B., and Ni, F. (2000) Identification of a thrombinbinding region in the sixth epidermal growth factor-like repeat of human thrombomodulin, Biochemistry 39, 10365-10372.

198. Song, J., Xu, P., Koutychenko, A., and Ni, F. (2002) Stability of protein-bound conformations of bioactive peptides: the folded conformation of an epidermal growth factor-like thrombomodulin fragment is similar to that recognized by thrombin, Biopolymers 65, 373-386.

199. Hrabal, R., Komives, E. A., and Ni, F. (1996) Structural resiliency of an EGF-like subdomain bound to its target protein, thrombin, Protein Sci 5, 195-203.

200. Marinescu, A., Cleary, D. B., Littlefield, T. R., and Maurer, M. C. (2002) Structural features associated with the binding of glutamine-containing peptides to Factor XIII, Arch Biochem Biophys 406, 9-20.

201. Bakhtiar, R., and Tse, F. L. (2000) Biological mass spectrometry: a primer, Mutagenesis 15, 415-430.

202. Hillenkamp, F., Karas, M., Beavis, R. C., and Chait, B. T. (1991) Matrix-assisted laser desorption/ionization mass spectrometry of biopolymers, Anal Chem 63, 1193A-1203A.

203. Zenobi, R., and Knochenmuss, R. (1998) Ion formation in MALDI mass spectrometry, Mass Spectrometry Reviews 17, 337-366.

204. Knochenmuss, R., and Zenobi, R. (2003) MALDI ionization: the role of in-plume processes, Chemical reviews 103, 441-452.

205. Dreisewerd, K. (2003) The desorption process in MALDI, Chemical reviews 103, $395-426$.

206. Park, M. and Callahan, J. (1994) An inductive detector for time-of-flight mass spectrometry, Rapid Commun. Mass Spectrom. 8, 317-322. 
207. Fuerstenau, S., and Benner, W. (1995) Molecular weight determination of megaDalton DNA electrospray ions using charge detection time-of-flight mass spectrometry, Rapid Commun. Mass Spectrom. 9, 1528-1538.

208. Glückmann, M., and Karas, M. (1999) The initial ion velocity and its dependence on matrix, analyte and preparation method in ultraviolet matrix-assisted laser desorption/ionization, Journal of Mass Spectrometry 34, 467-477.

209. Berkenkamp, S., Menzel, C., Hillenkamp, F., and Dreisewerd, K. (2002) Measurements of mean initial velocities of analyte and matrix ions in infrared matrix-assisted laser desorption ionization mass spectrometry, Journal of the American Society for Mass Spectrometry 13, 209-220.

210. Vestal, M., Juhasz, P., and Martin, S. (1995) Delayed extraction matrix-assisted laser desorption time-of-flight mass spectrometry, Rapid Commun. Mass Spectrom. 9, 1044-1050.

211. Takach, E. J., Hines, W. M., Patterson, D. H., Juhasz, P., Falick, A. M., Vestal, M. L., and Martin, S. A. (1997) Accurate mass measurements using MALDI-TOF with delayed extraction, Journal of protein chemistry 16, 363-369.

212. Cornish, T., and Cotter, R. (1993) A curved-field reflectron for improved energy focusing of product ions in time-of-flight mass spectrometry, Rapid Commun. Mass Spectrom. 7, 1037-1040.

213. Mamyrin, B., Karataev, V., Shmikk, D., and Zagulin, V. (1973) The massreflectron, a new nonmagnetic time-of-flight mass spectrometer with high resolution Sov. Phys. JETP 37, 45.

214. Spengler, B., Kirsch, D., Kaufmann, R., and Cotter, R. (1991) Metastable decay of peptides and proteins in matrix-assisted laser-desorption mass spectrometry, Rapid Commun. Mass Spectrom. 5, 198-202.

215. Apuy, J. L., Park, Z. Y., Swartz, P. D., Dangott, L. J., Russell, D. H., and Baldwin, T. O. (2001) Pulsed-alkylation mass spectrometry for the study of protein folding and dynamics: development and application to the study of a folding/unfolding intermediate of bacterial luciferase, Biochemistry 40, 1515315163. 
216. Glocker, M. O., Borchers, C., Fiedler, W., Suckau, D., and Przybylski, M. (1994) Molecular characterization of surface topology in protein tertiary structures by amino-acylation and mass spectrometric peptide mapping, Bioconjug Chem 5, 583-590.

217. D'Ambrosio, C., Talamo, F., Vitale, R. M., Amodeo, P., Tell, G., Ferrara, L., and Scaloni, A. (2003) Probing the dimeric structure of porcine aminoacylase 1 by mass spectrometric and modeling procedures, Biochemistry 42, 4430-4443.

218. Ansong, C., Miles, S. M., and Fay, P. J. (2006) Factor VIII A1 domain residues 97-105 represent a light chain-interactive site, Biochemistry 45, 13140-13149.

219. Hvidt, A., and Linderstrom-Lang, K. (1954) Exchange of hydrogen atoms in insulin with deuterium atoms in aqueous solutions, Biochim Biophys Acta 14, 574-575.

220. Barksdale, A. D., and Rosenberg, A. (1982) Acquisition and interpretation of hydrogen exchange data from peptides, polymers, and proteins, Methods of biochemical analysis 28, 1-113.

221. Englander, S. W., Downer, N. W., and Teitelbaum, H. (1972) Hydrogen exchange, Annu Rev Biochem 41, 903-924.

222. Hvidt, A., Johansen, G., and Linderstrom-Lang, K. (1960) Deuterium and 180 exchange, Pergamon, New York.

223. Resing, K. A., Hoofnagle, A. N., and Ahn, N. G. (1999) Modeling deuterium exchange behavior of ERK2 using pepsin mapping to probe secondary structure, $J$ Am Soc Mass Spectrom 10, 685-702.

224. Bai, Y., Milne, J. S., Mayne, L., and Englander, S. W. (1993) Primary structure effects on peptide group hydrogen exchange, Proteins 17, 75-86.

225. Englander, J. J., Rogero, J. R., and Englander, S. W. (1985) Protein hydrogen exchange studied by the fragment separation method, Analytical biochemistry 147, 234-244. 
226. Arrington, C. B., and Robertson, A. D. (2000) Correlated motions in native proteins from MS analysis of $\mathrm{NH}$ exchange: evidence for a manifold of unfolding reactions in ovomucoid third domain, $J$ Mol Biol 300, 221-232.

227. Swint-Kruse, L., and Robertson, A. D. (1996) Temperature and $\mathrm{pH}$ dependences of hydrogen exchange and global stability for ovomucoid third domain, Biochemistry 35, 171-180.

228. Clarke, J., and Itzhaki, L. S. (1998) Hydrogen exchange and protein folding, Current opinion in structural biology 8, 112-118.

229. Hoofnagle, A. N., Resing, K. A., and Ahn, N. G. (2003) Protein analysis by hydrogen exchange mass spectrometry, Annu Rev Biophys Biomol Struct 32, 1-25.

230. Mandell, J. G., Falick, A. M., and Komives, E. A. (1998) Measurement of amide hydrogen exchange by MALDI-TOF mass spectrometry, Anal Chem 70, 39873995.

231. Mandell, J. G., Falick, A. M., and Komives, E. A. (1998) Identification of proteinprotein interfaces by decreased amide proton solvent accessibility, Proc Natl Acad Sci U S A 95, 14705-14710.

232. Dunn, B. M. (2002) Structure and mechanism of the pepsin-like family of aspartic peptidases, Chemical reviews 102, 4431-4458.

233. Croy, C. H., Koeppe, J. R., Bergqvist, S., and Komives, E. A. (2004) Allosteric changes in solvent accessibility observed in thrombin upon active site occupation, Biochemistry 43, 5246-5255.

234. Koeppe, J. R., and Komives, E. A. (2006) Amide H/2H exchange reveals a mechanism of thrombin activation, Biochemistry 45, 7724-7732.

235. Getz, E. B., Xiao, M., Chakrabarty, T., Cooke, R., and Selvin, P. R. (1999) A comparison between the sulfhydryl reductants tris(2-carboxyethyl)phosphine and dithiothreitol for use in protein biochemistry, Analytical biochemistry 273, 73-80.

236. Hunt, D. F., Henderson, R. A., Shabanowitz, J., Sakaguchi, K., Michel, H., Sevilir, N., Cox, A. L., Appella, E., and Engelhard, V. H. (1992) Characterization 
of peptides bound to the class I MHC molecule HLA-A2.1 by mass spectrometry, Science 255, 1261-1263.

237. Coplen, T. B., Bohlke, J. K., De Bievre, P., Ding, T., Holden, N. E., Hopple, J. A., Krouse, H. R., Lamberty, A., Peiser, H. S., Revesz, K., Rieder, S. E., Rosmans, K. J. R., Roth, E., Taylor, P. D. P., Vocke, R. D., and Xiao, Y. K. (2002) Isotopeabundance variations of selected elements, Pure Appl. Chem. 74, 1987-2017.

238. Chen, J., and Smith, D. L. (2001) Amide hydrogen exchange shows that malate dehydrogenase is a folded monomer at pH 5, Protein Sci 10, 1079-1083.

239. Wang, L., Lane, L. C., and Smith, D. L. (2001) Detecting structural changes in viral capsids by hydrogen exchange and mass spectrometry, Protein Sci 10, 12341243.

240. Lane, D. A., Philippou, H., and Huntington, J. A. (2005) Directing thrombin, Blood 106, 2605-2612.

241. Stone, S. R., and Hofsteenge, J. (1986) Kinetics of the inhibition of thrombin by hirudin, Biochemistry 25, 4622-4628.

242. Pineda, A. O., Chen, Z. W., Marino, F., Mathews, F. S., Mosesson, M. W., and Di Cera, E. (2006) Crystal structure of thrombin in complex with fibrinogen gamma' peptide, Biophys Chem 125, 556-559.

243. Trumbo, T. A., and Maurer, M. C. (2000) Examining thrombin hydrolysis of the factor XIII activation peptide segment leads to a proposal for explaining the cardioprotective effects observed with the factor XIII V34L mutation, $J$ Biol Chem 275, 20627-20631.

244. Winzor, D. J., and Scheraga, H. A. (1964) Titration Behavior of Bovine Thrombin, Arch Biochem Biophys 104, 202-207.

245. Ni, F., Konishi, Y., Bullock, L. D., Rivetna, M. N., and Scheraga, H. A. (1989) High-resolution NMR studies of fibrinogen-like peptides in solution: structural basis for the bleeding disorder caused by a single mutation of $\operatorname{Gly}(12)$ to $\operatorname{Val}(12)$ in the A alpha chain of human fibrinogen Rouen, Biochemistry 28, 3106-3119. 
246. Ni, F., Konishi, Y., Frazier, R. B., Scheraga, H. A., and Lord, S. T. (1989) Highresolution NMR studies of fibrinogen-like peptides in solution: interaction of thrombin with residues 1-23 of the A alpha chain of human fibrinogen, Biochemistry 28, 3082-3094.

247. Mathews, II, Padmanabhan, K. P., Ganesh, V., Tulinsky, A., Ishii, M., Chen, J., Turck, C. W., Coughlin, S. R., and Fenton, J. W., 2nd. (1994) Crystallographic structures of thrombin complexed with thrombin receptor peptides: existence of expected and novel binding modes, Biochemistry 33, 3266-3279.

248. Martin, P. D., Robertson, W., Turk, D., Huber, R., Bode, W., and Edwards, B. F. (1992) The structure of residues 7-16 of the A alpha-chain of human fibrinogen bound to bovine thrombin at 2.3-A resolution, J Biol Chem 267, 7911-7920.

249. Wüthrich, K. (1986) NMR of Proteins and Nucleic Acids, Wiley, New York, NY.

250. He, X., Ye, J., Esmon, C. T., and Rezaie, A. R. (1997) Influence of Arginines 93, 97, and 101 of thrombin to its functional specificity, Biochemistry 36, 8969-8976.

251. Cristofaro, R., Carotti, A., Akhavan, S., Palla, R., Peyvandi, F., Altomare, C., and Mannucci, P. M. (2006) The natural mutation by deletion of Lys9 in the thrombin A-chain affects the pK value of catalytic residues, the overall enzyme's stability and conformational transitions linked to Na binding, Febs J 273, 159-169.

252. Marchese, P., Murata, M., Mazzucato, M., Pradella, P., De Marco, L., Ware, J., and Ruggeri, Z. M. (1995) Identification of three tyrosine residues of glycoprotein $\mathrm{Ib}$ alpha with distinct roles in von Willebrand factor and alpha-thrombin binding, J Biol Chem 270, 9571-9578.

253. Olsen, P. H., Esmon, N. L., Esmon, C. T., and Laue, T. M. (1992) Ca2+ dependence of the interactions between protein $\mathrm{C}$, thrombin, and the elastase fragment of thrombomodulin. Analysis by ultracentrifugation, Biochemistry 31, 746-754.

254. McGowan, E. B., Ding, A., and Detwiler, T. C. (1983) Correlation of thrombininduced glycoprotein $\mathrm{V}$ hydrolysis and platelet activation, $J$ Biol Chem 258, 11243-11248.

255. Vindigni, A., White, C. E., Komives, E. A., and Di Cera, E. (1997) Energetics of thrombin-thrombomodulin interaction, Biochemistry 36, 6674-6681. 
256. de Cristofaro, R., de Candia, E., Croce, G., Morosetti, R., and Landolfi, R. (1998) Binding of human alpha-thrombin to platelet GpIb: energetics and functional effects, Biochem J 332 ( Pt 3), 643-650.

257. Bouton, M. C., Jandrot-Perrus, M., Moog, S., Cazenave, J. P., Guillin, M. C., and Lanza, F. (1995) Thrombin interaction with a recombinant N-terminal extracellular domain of the thrombin receptor in an acellular system, Biochem $J$ 305 ( Pt 2), 635-641.

258. Martin, P. D., Malkowski, M. G., DiMaio, J., Konishi, Y., Ni, F., and Edwards, B. F. (1996) Bovine thrombin complexed with an uncleavable analog of residues 719 of fibrinogen A alpha: geometry of the catalytic triad and interactions of the P1', P2', and P3' substrate residues, Biochemistry 35, 13030-13039.

259. Vitali, J., Martin, P. D., Malkowski, M. G., Robertson, W. D., Lazar, J. B., Winant, R. C., Johnson, P. H., and Edwards, B. F. (1992) The structure of a complex of bovine alpha-thrombin and recombinant hirudin at 2.8-A resolution, $J$ Biol Chem 267, 17670-17678.

260. Fuentes-Prior, P., Iwanaga, Y., Huber, R., Pagila, R., Rumennik, G., Seto, M., Morser, J., Light, D. R., and Bode, W. (2000) Structural basis for the anticoagulant activity of the thrombin-thrombomodulin complex, Nature 404, 518-525.

261. Martin, B. M., Wasiewski, W. W., Fenton, J. W., 2nd, and Detwiler, T. C. (1976) Equilibrium binding of thrombin to platelets, Biochemistry 15, 4886-4893.

262. Workman, E. F., Jr., White, G. C., 2nd, and Lundblad, R. L. (1977) Structurefunction relationships in the interaction of alpha-thrombin with blood platelets, $J$ Biol Chem 252, 7118-7123.

263. Berndt, M. C., Gregory, C., Kabral, A., Zola, H., Fournier, D., and Castaldi, P. A. (1985) Purification and preliminary characterization of the glycoprotein Ib complex in the human platelet membrane, Eur J Biochem 151, 637-649.

264. De Marco, L., Mazzucato, M., Masotti, A., Fenton, J. W., 2nd, and Ruggeri, Z. M. (1991) Function of glycoprotein Ib alpha in platelet activation induced by alphathrombin, $J$ Biol Chem 266, 23776-23783. 
265. Adam, F., Bouton, M. C., Huisse, M. G., and Jandrot-Perrus, M. (2003) Thrombin interaction with platelet membrane glycoprotein Ib alpha, Trends Mol Med 9, 461-464.

266. Brocklehurst, K., and Malthouse, J. P. (1978) Mechanism of the reaction of papain with substrate-derived diazomethyl ketones. Implications for the difference in site specificity of halomethyl ketones for serine proteinases and cysteine proteinases and for stereoelectronic requirements in the papain catalytic mechanism, Biochem J 175, 761-764.

267. Bishop, P. D., Teller, D. C., Smith, R. A., Lasser, G. W., Gilbert, T., and Seale, R. L. (1990) Expression, purification, and characterization of human factor XIII in Saccharomyces cerevisiae, Biochemistry 29, 1861-1869.

268. Rajagopal, S., Meyer, S. C., Goldman, A., Zhou, M., and Ghosh, I. (2006) A minimalist approach toward protein recognition by epitope transfer from functionally evolved beta-sheet surfaces, $J$ Am Chem Soc 128, 14356-14363.

269. Hornyak, T. J., and Shafer, J. A. (1992) Interactions of factor XIII with fibrin as substrate and cofactor, Biochemistry 31, 423-429.

270. Okada, M., Blomback, B., Chang, M. D., and Horowitz, B. (1985) Fibronectin and fibrin gel structure, $J$ Biol Chem 260, 1811-1820.

271. Mikkola, H., Yee, V. C., Syrjala, M., Seitz, R., Egbring, R., Petrini, P., Ljung, R., Ingerslev, J., Teller, D. C., Peltonen, L., and Palotie, A. (1996) Four novel mutations in deficiency of coagulation factor XIII: consequences to expression and structure of the A-subunit, Blood 87, 141-151.

272. Onland, W., Boing, A. N., Meijer, A. B., Schaap, M. C., Nieuwland, R., Haasnoot, K., Sturk, A., and Peters, M. (2005) Congenital deficiency of factor XIII caused by two missense mutations in a Dutch family, Haemophilia 11, 539547.

273. Mikkola, H., Syrjala, M., Rasi, V., Vahtera, E., Hamalainen, E., Peltonen, L., and Palotie, A. (1994) Deficiency in the A-subunit of coagulation factor XIII: two novel point mutations demonstrate different effects on transcript levels, Blood 84, 517-525. 
274. Vysokovsky, A., Saxena, R., Landau, M., Zivelin, A., Eskaraev, R., Rosenberg, N., Seligsohn, U., and Inbal, A. (2004) Seven novel mutations in the factor XIII A-subunit gene causing hereditary factor XIII deficiency in 10 unrelated families, J Thromb Haemost 2, 1790-1797.

275. Peyvandi, F., Tagliabue, L., Menegatti, M., Karimi, M., Komaromi, I., Katona, E., Muszbek, L., and Mannucci, P. M. (2004) Phenotype-genotype characterization of 10 families with severe a subunit factor XIII deficiency, Hum Mutat 23, 98.

276. Ichinose, A., Tsukamoto, H., Izumi, T., Yamazaki, T., Togashi, M., Takamatsu, J., Saito, H., and Umeyama, H. (1998) Arg260-Cys mutation in severe factor XIII deficiency: conformational change of the A subunit is predicted by molecular modelling and mechanics, Br J Haematol 101, 264-272.

277. Kangsadalampai, S., Chelvanayagam, G., Baker, R., Tiedemann, K., Kuperan, P., and Board, P. G. (1999) Identification and characterization of two missense mutations causing factor XIIIA deficiency, Br J Haematol 104, 37-43.

278. Weiss, M. S., Metzner, H. J., and Hilgenfeld, R. (1998) Two non-proline cis peptide bonds may be important for factor XIII function, FEBS Lett 423, 291-296.

279. Boeshans, K. M., Mueser, T. C., and Ahvazi, B. (2007) A three-dimensional model of the human transglutaminase 1: insights into the understanding of lamellar ichthyosis, J Mol Model (Online) 13, 233-246.

280. Ahvazi, B., and Steinert, P. M. (2003) A model for the reaction mechanism of the transglutaminase 3 enzyme, Exp Mol Med 35, 228-242.

281. Laiho, E., Ignatius, J., Mikkola, H., Yee, V. C., Teller, D. C., Niemi, K. M., Saarialho-Kere, U., Kere, J., and Palotie, A. (1997) Transglutaminase 1 mutations in autosomal recessive congenital ichthyosis: private and recurrent mutations in an isolated population, Am J Hum Genet 61, 529-538.

282. Anwar, R., Gallivan, L., Edmonds, S. D., and Markham, A. F. (1999) Genotype/phenotype correlations for coagulation factor XIII: specific normal polymorphisms are associated with high or low factor XIII specific activity, Blood 93, 897-905.

283. Lewis, E. A., and Murphy, K. P. (2005) Isothermal titration calorimetry, Methods Mol Biol 305, 1-16. 
284. Jelesarov, I., and Bosshard, H. R. (1999) Isothermal titration calorimetry and differential scanning calorimetry as complementary tools to investigate the energetics of biomolecular recognition, J Mol Recognit 12, 3-18.

285. Dunitz, J. D. (1995) Win some, lose some: enthalpy-entropy compensation in weak intermolecular interactions, Chem Biol 2, 709-712.

286. Clemetson, K. J., and Clemetson, J. M. (1995) Platelet GPIb-V-IX complex. Structure, function, physiology, and pathology, Semin Thromb Hemost 21, 130136.

287. Beer, J. H., Buchi, L., and Steiner, B. (1994) Glycocalicin: a new assay--the normal plasma levels and its potential usefulness in selected diseases, Blood 83, 691-702.

288. Sturtevant, J. M. (1977) Heat capacity and entropy changes in processes involving proteins, Proc Natl Acad Sci US A 74, 2236-2240.

289. Baerga-Ortiz, A., Bergqvist, S., Mandell, J. G., and Komives, E. A. (2004) Two different proteins that compete for binding to thrombin have opposite kinetic and thermodynamic profiles, Protein Sci 13, 166-176.

290. Wood, M. J., Sampoli Benitez, B. A., and Komives, E. A. (2000) Solution structure of the smallest cofactor-active fragment of thrombomodulin, Nat Struct Biol 7, 200-204.

291. Ren, J., Jenkins, T. C., and Chaires, J. B. (2000) Energetics of DNA intercalation reactions, Biochemistry 39, 8439-8447.

292. Zingali, R. B., Jandrot-Perrus, M., Guillin, M. C., and Bon, C. (1993) Bothrojaracin, a new thrombin inhibitor isolated from Bothrops jararaca venom: characterization and mechanism of thrombin inhibition, Biochemistry 32, 1079410802 .

293. Monteiro, R. Q., Bock, P. E., Bianconi, M. L., and Zingali, R. B. (2001) Characterization of bothrojaracin interaction with human prothrombin, Protein Sci 10, 1897-1904. 
294. Stejskal, E. O. a. T., J.E. (1965) Spin diffusion measurements: spin echoes in the presence of a time-dependent field gradient, J Chem Phys 42, 288-292.

295. Lapham, J., Rife, J. P., Moore, P. B., and Crothers, D. M. (1997) Measurement of diffusion constants for nucleic acids by NMR, Journal of biomolecular NMR 10, 255-262.

296. Dingley, A. J., Mackay, J. P., Chapman, B. E., Morris, M. B., Kuchel, P. W., Hambly, B. D., and King, G. F. (1995) Measuring protein self-association using pulsed-field-gradient NMR spectroscopy: application to myosin light chain 2, Journal of biomolecular NMR 6, 321-328.

297. Mosesson, M. W., Hernandez, I., Raife, T. J., Medved, L., Yakovlev, S., Simpson-Haidaris, P. J., Uitte, D. E. W. S., and Bertina, R. M. (2007) Plasma fibrinogen gamma' chain content in the thrombotic microangiopathy syndrome, $J$ Thromb Haemost 5, 62-69.

298. Harmon, J. T., and Jamieson, G. A. (1986) The glycocalicin portion of platelet glycoprotein $\mathrm{Ib}$ expresses both high and moderate affinity receptor sites for thrombin. A soluble radioreceptor assay for the interaction of thrombin with platelets, J Biol Chem 261, 13224-13229.

299. Baerga-Ortiz, A., Hughes, C. A., Mandell, J. G., and Komives, E. A. (2002) Epitope mapping of a monoclonal antibody against human thrombin by H/Dexchange mass spectrometry reveals selection of a diverse sequence in a highly conserved protein, Protein Sci 11, 1300-1308.

300. Truhlar, S. M., Torpey, J. W., and Komives, E. A. (2006) Regions of IkappaBalpha that are critical for its inhibition of NF-kappaB.DNA interaction fold upon binding to NF-kappaB, Proc Natl Acad Sci U S A 103, 18951-18956.

301. Kusch, M., Grundmann, C., Keitel, S., Seitz, R., and Konig, H. (2006) A novel assay for factor XIII based on cross-linking of synthetic peptides: analysis of different substrates, Blood Coagul Fibrinolysis 17, 575-580. 


\section{APPENDIX A CHEMICAL SHIFT TABLES}

\section{(All Samples in Aqueous Solution and Referenced to EDTA)}

Table A: ${ }^{1} \mathrm{H}$ Resonance Assignments (ppm) of the $\gamma^{\prime}$ peptide $\left(\mathrm{Y}_{\mathrm{P}}^{418} \mathrm{Y}_{\mathrm{P}}{ }^{422}\right)$ at $15^{\circ} \mathrm{C}$.

\begin{tabular}{|c|c|c|c|c|c|c|}
\hline Residue & $\mathrm{NH}$ & $\mathrm{C}^{\alpha} \mathrm{H}$ & $\mathrm{C}^{\beta} \mathrm{H}$ & $\mathrm{C}^{\gamma} \mathrm{H}$ & $\mathrm{C}^{\delta} \mathrm{H}$ & $\mathrm{C}^{\varepsilon} \mathrm{H}$ \\
\hline $\mathrm{P}^{410}$ & & 4.40 & 2.45 & $2.07,2.01$ & $3.42,3.39$ & \\
\hline$E^{411}$ & 8.86 & 4.25 & $1.95,1.88$ & $2.26,2.20$ & & \\
\hline $\mathrm{H}^{412}$ & 8.64 & 5.00 & $3.22,3.13$ & & 7.31 & \\
\hline $\mathrm{P}^{413}$ & & 4.43 & 2.32 & $2.02,1.98$ & $3.76,3.58$ & \\
\hline$A^{414}$ & 8.61 & 4.31 & 1.43 & & & \\
\hline$E^{415}$ & 8.57 & 4.35 & $2.11,1.99$ & 2.32 & & \\
\hline $\mathrm{T}^{416}$ & 8.18 & 4.33 & 4.17 & 1.13 & & \\
\hline$E^{417}$ & 8.49 & 4.28 & $1.96,1.86$ & $2.20,2.15$ & & \\
\hline$Y_{P}^{418}$ & 8.26 & 4.59 & $3.13,2.94$ & & 7.19 & 7.14 \\
\hline$D^{419}$ & 8.35 & 4.60 & $2.70,2.60$ & & & \\
\hline$S^{420}$ & 8.28 & 4.36 & $3.84,3.79$ & & & \\
\hline $\mathrm{L}^{421}$ & 8.29 & 4.25 & 1.52 & 1.38 & $0.88,0.81$ & \\
\hline $\mathrm{Y}_{\mathrm{P}}^{422}$ & 8.26 & $?$ & $3.13,2.86$ & & 7.23 & 7.13 \\
\hline $\mathrm{P}^{423}$ & & 4.42 & 2.29 & $2.03,1.99$ & $3.72,3.62$ & \\
\hline$E^{424}$ & $8.72,8.65$ & 4.31 & $2.10,1.96$ & 2.36 & & \\
\hline$D^{425}$ & 8.38 & 4.63 & $2.71,2.64$ & & & \\
\hline$D^{426}$ & 8.36 & 4.67 & $2.76,2.62$ & & & \\
\hline $\mathrm{L}^{427}$ & 7.80 & 4.17 & 1.62 & 1.58 & $0.92,0.86$ & \\
\hline
\end{tabular}

Table B: ${ }^{1} \mathrm{H}$ Resonance Assignments (ppm) of the $\gamma^{\prime}$ peptide $\left(\mathrm{Y}_{\mathrm{P}}{ }^{418} \mathrm{Y}_{\mathrm{P}}{ }^{422}\right.$ ) bound to IIa at $15^{\circ} \mathrm{C}$. The assignments for this peptide bound to $\gamma$-IIa are not significantly different.

\begin{tabular}{ccccccc} 
Residue & $\mathrm{NH}$ & $\mathrm{C}^{\alpha} \mathrm{H}$ & $\mathrm{C}^{\beta} \mathrm{H}$ & $\mathrm{C}^{\gamma} \mathrm{H}$ & $\mathrm{C}^{\delta} \mathrm{H}$ & $\mathrm{C}^{\varepsilon} \mathrm{H}$ \\
\hline $\mathrm{P}^{410}$ & & 4.40 & 2.45 & $2.07,2.00$ & $3.42,3.38$ & \\
$\mathrm{E}^{411}$ & 8.86 & 4.25 & $1.96,1.88$ & $2.25,2.20$ & \\
$\mathrm{H}^{412}$ & 8.66 & 4.99 & $3.23,3.13$ & & 7.31 & \\
$\mathrm{P}^{413}$ & & 4.43 & 2.31 & $2.02,1.98$ & $3.76,3.58$ \\
$\mathrm{~A}^{414}$ & 8.61 & 4.32 & 1.42 & & \\
$\mathrm{E}^{415}$ & 8.57 & 4.34 & $2.11,1.98$ & 2.32 & \\
$\mathrm{~T}^{416}$ & 8.19 & 4.32 & 4.17 & 1.13 & \\
$\mathrm{E}^{417}$ & 8.49 & 4.28 & $1.96,1.86$ & $2.21,2.13$ & & 7.08 \\
$\mathrm{Y}_{\mathrm{P}}^{418}$ & 8.25 & 4.60 & $3.14,2.93$ & & & \\
$\mathrm{D}^{419}$ & 8.36 & 4.59 & $2.70,2.61$ & & & \\
$\mathrm{~S}^{420}$ & 8.26 & 4.35 & $3.83,3.79$ & & & \\
$\mathrm{~L}^{421}$ & 8.29 & 4.28 & 1.52 & 1.38 & $0.88,0.81$ & \\
$\mathrm{Y}_{\mathrm{P}}^{422}$ & 8.26 & 4.86 & $3.14,2.85$ & & $3.72,3.62$ & \\
$\mathrm{P}^{423}$ & & 4.42 & 2.29 & $2.03,1.99$ & & \\
$\mathrm{E}^{424}$ & $8.73,8.70$ & 4.32 & $2.11,1.97$ & 2.35 & & \\
$\mathrm{D}^{425}$ & 8.35 & 4.63 & $2.71,2.65$ & & & \\
$\mathrm{D}^{426}$ & 8.34 & 4.67 & $2.76,2.61$ & & & \\
$\mathrm{~L}^{427}$ & 7.79 & 4.17 & 1.64 & 1.57 & $0.91,0.86$ &
\end{tabular}


Table C: ${ }^{1} \mathrm{H}$ Resonance Assignments (ppm) of the $\gamma^{\prime}$ peptide $\left(\mathrm{Y}_{\mathrm{P}}{ }^{418}\right)$ at $15^{\circ} \mathrm{C}$.

\begin{tabular}{ccccccc} 
Residue & $\mathrm{NH}$ & $\mathrm{C}^{\alpha} \mathrm{H}$ & $\mathrm{C}^{\beta} \mathrm{H}$ & $\mathrm{C}^{\gamma} \mathrm{H}$ & $\mathrm{C}^{\delta} \mathrm{H}$ & $\mathrm{C}^{\varepsilon} \mathrm{H}$ \\
\hline $\mathrm{P}^{410}$ & & 4.40 & 2.47 & $2.10,2.03$ & $3.43,3.39$ & \\
$\mathrm{E}^{411}$ & 8.86 & 4.25 & $1.97,1.88$ & $2.24,2.18$ & & \\
$\mathrm{H}^{412}$ & 8.64 & 5.02 & $3.23,3.13$ & & 7.30 & \\
$\mathrm{P}^{413}$ & & 4.43 & 2.34 & 2.02 & $3.76,3.57$ & \\
$\mathrm{~A}^{414}$ & 8.61 & 4.31 & 1.43 & & & \\
$\mathrm{E}^{415}$ & 8.57 & 4.35 & 2.12 & $2.32,2.00$ & & \\
$\mathrm{~T}^{416}$ & 8.19 & 4.33 & 4.17 & 1.14 & & \\
$\mathrm{E}^{417}$ & 8.50 & 4.28 & $1.99,1.88$ & $2.20,2.15$ & & \\
$\mathrm{Y}_{\mathrm{P}}^{418}$ & 8.27 & 4.59 & $3.13,2.96$ & & 7.18 & 7.11 \\
$\mathrm{D}^{419}$ & 8.34 & 4.60 & $2.70,2.60$ & & & \\
$\mathrm{~S}^{420}$ & 8.21 & 4.36 & $3.84,3.79$ & & & \\
$\mathrm{~L}^{421}$ & 8.20 & 4.24 & 1.48 & 1.31 & $0.87,0.79$ & \\
$\mathrm{Y}^{422}$ & 8.18 & 4.86 & $3.08,2.83$ & & 7.15 & 6.82 \\
$\mathrm{P}^{423}$ & & 4.42 & 2.28 & 2.00 & $3.69,3.57$ & \\
$\mathrm{E}^{424}$ & 8.61 & 4.31 & $2.12,1.96$ & 2.36 & & \\
$\mathrm{D}^{425}$ & 8.36 & 4.63 & $2.74,2.65$ & & & \\
$\mathrm{D}^{426}$ & 8.34 & 4.66 & $2.76,2.61$ & & & \\
$\mathrm{~L}^{427}$ & 7.79 & 4.16 & 1.57 & $1.63,1.60$ & $0.92,0.86$ &
\end{tabular}

Table D: ${ }^{1} \mathrm{H}$ Resonance Assignments (ppm) of the $\gamma^{\prime}$ peptide $\left(\mathrm{Y}_{\mathrm{P}}{ }^{418}\right)$ bound to IIa at $15^{\circ} \mathrm{C}$.

\begin{tabular}{ccccccc} 
Residue & $\mathrm{NH}$ & $\mathrm{C}^{\alpha} \mathrm{H}$ & $\mathrm{C}^{\beta} \mathrm{H}$ & $\mathrm{C}^{\gamma} \mathrm{H}$ & $\mathrm{C}^{\delta} \mathrm{H}$ & $\mathrm{C}^{\varepsilon} \mathrm{H}$ \\
\hline $\mathrm{P}^{410}$ & & 4.40 & 2.45 & $2.08,2.03$ & $3.42,3.38$ & \\
$\mathrm{E}^{411}$ & 8.86 & 4.25 & $1.96,1.89$ & $2.21,2.26$ & & \\
$\mathrm{H}^{412}$ & 8.66 & 4.99 & $3.22,3.13$ & & 7.30 & \\
$\mathrm{P}^{413}$ & & 4.43 & 2.32 & $2.03,1.99$ & $3.77,3.58$ & \\
$\mathrm{~A}^{414}$ & 8.61 & 4.33 & 1.43 & & & \\
$\mathrm{E}^{415}$ & 8.58 & 4.36 & $2.11,1.99$ & 2.32 & & \\
$\mathrm{~T}^{416}$ & 8.20 & 4.36 & 4.17 & 1.14 & & \\
$\mathrm{E}^{417}$ & 8.50 & 4.29 & $1.97,1.87$ & $2.21,2.14$ & & \\
$\mathrm{Y}_{\mathrm{P}}^{418}$ & 8.25 & 4.60 & $3.13,2.95$ & & 7.18 & 7.09 \\
$\mathrm{D}^{419}$ & 8.34 & 4.60 & $2.70,2.62$ & & & \\
$\mathrm{~S}^{420}$ & 8.21 & 4.36 & $3.85,3.79$ & & & \\
$\mathrm{~L}^{421}$ & 8.20 & 4.24 & 1.47 & 1.32 & $0.87,0.79$ & \\
$\mathrm{Y}^{422}$ & 8.19 & 4.86 & $3.09,2.81$ & & 7.14 & 6.80 \\
$\mathrm{P}^{423}$ & & 4.42 & 2.28 & $2.01,1.98$ & $3.69,3.57$ & \\
$\mathrm{E}^{424}$ & 8.63 & 4.31 & $2.10,1.96$ & 2.36 & & \\
$\mathrm{D}^{425}$ & 8.35 & 4.64 & $2.72,2.67$ & & & \\
$\mathrm{D}^{426}$ & 8.33 & 4.68 & $2.75,2.62$ & & & \\
$\mathrm{~L}^{427}$ & 7.79 & 4.17 & 1.62 & 1.57 & $0.92,0.86$ &
\end{tabular}


Table E: ${ }^{1} \mathrm{H}$ Resonance Assignments (ppm) of the $\gamma^{\prime}$ peptide $\left(\mathrm{Y}_{\mathrm{P}}{ }^{422}\right)$ at $15^{\circ} \mathrm{C}$.

\begin{tabular}{ccccccc} 
Residue & $\mathrm{NH}$ & $\mathrm{C}^{\alpha} \mathrm{H}$ & $\mathrm{C}^{\beta} \mathrm{H}$ & $\mathrm{C}^{\gamma} \mathrm{H}$ & $\mathrm{C}^{\delta} \mathrm{H}$ & $\mathrm{C}^{\varepsilon} \mathrm{H}$ \\
\hline $\mathrm{P}^{410}$ & & 4.40 & 2.45 & $2.07,2.00$ & $3.42,3.39$ & \\
$\mathrm{E}^{411}$ & 8.85 & 4.25 & $1.96,1.88$ & $2.25,2.20$ & & \\
$\mathrm{H}^{412}$ & 8.65 & 5.01 & $3.24,3.14$ & & 7.32 & \\
$\mathrm{P}^{413}$ & & 4.43 & 2.32 & $2.02,1.95$ & $3.76,3.58$ & \\
$\mathrm{~A}^{414}$ & 8.61 & 4.31 & 1.43 & & & \\
$\mathrm{E}^{415}$ & 8.57 & 4.36 & $2.11,1.99$ & 2.33 & & \\
$\mathrm{~T}^{416}$ & 8.18 & 4.32 & 4.16 & 1.13 & & \\
$\mathrm{E}^{417}$ & 8.47 & 4.28 & $1.96,1.87$ & $2.20,2.15$ & & \\
$\mathrm{Y}^{418}$ & 8.21 & 4.56 & $3.09,2.92$ & & 7.11 & 6.82 \\
$\mathrm{D}^{419}$ & 8.32 & 4.59 & $2.71,2.62$ & & & \\
$\mathrm{~S}^{420}$ & 8.17 & 4.34 & $3.85,3.79$ & & & \\
$\mathrm{~L}^{421}$ & 8.20 & 4.23 & 1.51 & 1.34 & $0.87,0.80$ & \\
$\mathrm{Y}^{4} 22$ & 8.18 & 4.85 & $3.13,2.84$ & & 7.21 & 7.12 \\
$\mathrm{P}^{423}$ & & 4.42 & 2.27 & $2.01,1.96$ & $3.67,3.59$ & \\
$\mathrm{E}^{424}$ & 8.64 & 4.32 & $2.10,1.96$ & 2.36 & & \\
$\mathrm{D}^{425}$ & 8.38 & 4.63 & $2.74,2.66$ & & & \\
$\mathrm{D}^{426}$ & 8.36 & 4.66 & $2.77,2.63$ & & & \\
$\mathrm{~L}^{427}$ & 7.80 & 4.18 & 1.64 & 1.57 & $0.91,0.87$ &
\end{tabular}

Table F: ${ }^{1} \mathrm{H}$ Resonance Assignments (ppm) of the $\gamma^{\prime}$ peptide $\left(\mathrm{Y}_{\mathrm{P}}{ }^{422}\right)$ bound to IIa at $15^{\circ} \mathrm{C}$.

\begin{tabular}{ccccccc} 
Residue & $\mathrm{NH}$ & $\mathrm{C}^{\alpha} \mathrm{H}$ & $\mathrm{C}^{\beta} \mathrm{H}$ & $\mathrm{C}^{\gamma} \mathrm{H}$ & $\mathrm{C}^{\delta} \mathrm{H}$ & $\mathrm{C}^{\varepsilon} \mathrm{H}$ \\
\hline $\mathrm{P}^{410}$ & & 4.40 & 2.45 & $2.08,2.00$ & $3.43,3.39$ & \\
$\mathrm{E}^{411}$ & 8.86 & 4.25 & $1.95,1.88$ & $2.24,2.19$ & & \\
$\mathrm{H}^{412}$ & 8.66 & 5.00 & $3.23,3.14$ & & 7.32 & \\
$\mathrm{P}^{413}$ & & 4.43 & 2.32 & $2.02,1.95$ & $3.76,3.58$ & \\
$\mathrm{~A}^{414}$ & 8.61 & 4.32 & 1.42 & & & \\
$\mathrm{E}^{415}$ & 8.57 & 4.35 & $2.11,1.98$ & 2.32 & & \\
$\mathrm{~T}^{416}$ & 8.20 & 4.33 & 4.18 & 1.14 & & \\
$\mathrm{E}^{417}$ & 8.47 & 4.26 & $1.97,1.88$ & $2.19,2.13$ & & \\
$\mathrm{Y}^{418}$ & 8.20 & 4.58 & $3.08,2.90$ & & 7.11 & 6.78 \\
$\mathrm{D}^{419}$ & 8.30 & 4.58 & $2.72,2.62$ & & & \\
$\mathrm{~S}^{420}$ & 8.20 & 4.32 & $3.84,3.79$ & & & \\
$\mathrm{~L}^{421}$ & 8.22 & 4.21 & 1.50 & 1.31 & $0.84,0.74$ & \\
$\mathrm{Y}_{\mathrm{P}}^{422}$ & 8.15 & 4.86 & $3.12,2.82$ & & 7.20 & 7.11 \\
$\mathrm{P}^{423}$ & & 4.42 & 2.27 & $2.01,1.95$ & $3.68,3.61$ & \\
$\mathrm{E}^{424}$ & 8.68 & 4.31 & $2.11,1.96$ & 2.36 & & \\
$\mathrm{D}^{425}$ & 8.36 & 4.64 & $2.73,2.67$ & & & \\
$\mathrm{D}^{426}$ & 8.33 & 4.68 & $2.75,2.61$ & & & \\
$\mathrm{~L}^{427}$ & 7.80 & 4.18 & 1.64 & 1.59 & $0.90,0.86$ &
\end{tabular}


Table G: ${ }^{1} \mathrm{H}$ Resonance Assignments (ppm) of the $\gamma^{\prime}$ peptide (No Phosphorylation) at $17^{\circ} \mathrm{C}$. In the presence of IIa, the peptide does not experience significant deviations in chemical shift.

\begin{tabular}{ccccccc} 
Residue & $\mathrm{NH}$ & $\mathrm{C}^{\alpha} \mathrm{H}$ & $\mathrm{C}^{\beta} \mathrm{H}$ & $\mathrm{C}^{\gamma} \mathrm{H}$ & $\mathrm{C}^{\delta} \mathrm{H}$ & $\mathrm{C}^{\varepsilon} \mathrm{H}$ \\
\hline $\mathrm{P}^{410}$ & & 4.41 & 2.46 & $2.08,2.00$ & $3.42,3.39$ & \\
$\mathrm{E}^{411}$ & 8.84 & 4.25 & $1.94,1.88$ & $2.25,2.19$ & & \\
$\mathrm{H}^{412}$ & 8.64 & 5.00 & $3.23,3.13$ & & 7.32 & \\
$\mathrm{P}^{413}$ & & 4.42 & 2.32 & $2.02,1.95$ & $3.74,3.57$ & \\
$\mathrm{~A}^{414}$ & 8.60 & 4.32 & 1.42 & & & \\
$\mathrm{E}^{415}$ & 8.55 & 4.34 & $2.08,1.98$ & 2.31 & & \\
$\mathrm{~T}^{416}$ & 8.19 & 4.31 & 4.16 & 1.13 & & \\
$\mathrm{E}^{417}$ & 8.46 & 4.26 & $1.96,1.87$ & $2.19,2.14$ & & \\
$\mathrm{Y}^{418}$ & 8.19 & 4.53 & $3.08,2.92$ & & 7.13 & 6.81 \\
$\mathrm{D}^{419}$ & 8.30 & 4.57 & $2.68,2.61$ & & & \\
$\mathrm{~S}^{420}$ & 8.15 & 4.34 & $3.85,3.80$ & & & \\
$\mathrm{~L}^{421}$ & 8.15 & 4.19 & 1.45 & 1.27 & $0.87,0.78$ & \\
$\mathrm{Y}_{\mathrm{P}}^{422}$ & 8.10 & $?$ & $3.07,2.81$ & & 7.14 & 6.81 \\
$\mathrm{P}^{423}$ & & 4.42 & 2.26 & $2.00,1.97$ & $3.65,3.55$ & \\
$\mathrm{E}^{424}$ & 8.59 & 4.28 & $2.08,1.94$ & 2.33 & & \\
$\mathrm{D}^{425}$ & 8.32 & 4.62 & $2.70,2.64$ & & & \\
$\mathrm{D}^{426}$ & 8.32 & 4.66 & $2.74,2.60$ & & & \\
$\mathrm{~L}^{427}$ & 7.78 & 4.15 & 1.61 & 1.58 & $0.91,0.86$ &
\end{tabular}

Table H: ${ }^{1} \mathrm{H}$ Resonance Assignments (ppm) of the GpIb $\alpha$ peptide $\left(\mathrm{Y}_{\mathrm{P}}{ }^{276} \mathrm{Y}_{\mathrm{P}}{ }^{279}\right)$ bound to IIa at $17^{\circ} \mathrm{C}$.

\begin{tabular}{|c|c|c|c|c|c|c|}
\hline Residue & $\mathrm{NH}$ & $\mathrm{C}^{\alpha} \mathrm{H}$ & $\mathrm{C}^{\beta} \mathrm{H}$ & $\mathrm{C}^{\gamma} \mathrm{H}$ & $\mathrm{C}^{\delta} \mathrm{H}$ & $\mathrm{C}^{\varepsilon} \mathrm{H}$ \\
\hline $\mathrm{D}^{269}$ & $?$ & $?$ & $?$ & & & \\
\hline$E^{270}$ & 8.95 & 4.27 & 2.30 & $2.06,1.99$ & & \\
\hline $\mathrm{G}^{271}$ & 8.67 & $4.03,3.96$ & & & & \\
\hline $\mathrm{D}^{272}$ & 8.14 & 4.69 & $2.75,2.73$ & & & \\
\hline $\mathrm{T}^{273}$ & 8.22 & 4.27 & 4.24 & 1.17 & & \\
\hline $\mathrm{D}^{274}$ & 8.44 & 4.61 & $2.71,2.63$ & & & \\
\hline $\mathrm{L}^{275}$ & 8.13 & 4.25 & 1.52 & 1.44 & $0.87,0.80$ & \\
\hline$Y_{P}{ }^{276}$ & 8.14 & 4.50 & $2.99,2.89$ & & 7.19 & 7.11 \\
\hline $\mathrm{D}^{277}$ & 8.23 & 4.55 & $2.60,2.54$ & & & \\
\hline $\mathrm{Y}^{278}$ & 7.97 & 4.49 & 2.86 & & 7.06 & 6.78 \\
\hline $\mathrm{Y}_{\mathrm{P}}^{279}$ & 8.20 & 4.75 & $3.05,2.83$ & & 7.20 & 7.18 \\
\hline $\mathrm{P}^{280}$ & & 4.34 & 2.26 & 1.95 & 3.47 & \\
\hline$E^{281}$ & 8.56 & 4.29 & 2.35 & $2.07,1.97$ & & \\
\hline $\mathrm{E}^{282}$ & 8.49 & 4.31 & 2.29 & $2.05,1.95$ & & \\
\hline $\mathrm{D}^{283}$ & 8.55 & 4.72 & $2.79,2.66$ & & & \\
\hline $\mathrm{T}^{284}$ & 8.22 & 4.37 & 4.29 & 1.22 & & \\
\hline $\mathrm{E}^{285}$ & 8.46 & 4.36 & 2.31 & $2.13,1.98$ & & \\
\hline $\mathrm{G}^{286}$ & 8.11 & 3.78 & & & & \\
\hline
\end{tabular}


Table I: ${ }^{1} \mathrm{H}$ Resonance Assignments (ppm) of the GpIb $\alpha$ peptide (No Phosphorylation) at $17^{\circ} \mathrm{C}$.

\begin{tabular}{ccccccc} 
Residue & $\mathrm{NH}$ & $\mathrm{C}^{\alpha} \mathrm{H}$ & $\mathrm{C}^{\beta} \mathrm{H}$ & $\mathrm{C}^{\gamma} \mathrm{H}$ & $\mathrm{C}^{\delta} \mathrm{H}$ & $\mathrm{C}^{\varepsilon} \mathrm{H}$ \\
\hline $\mathrm{D}^{269}$ & $?$ & $?$ & $?$ & & & \\
$\mathrm{E}^{270}$ & 8.94 & 4.28 & 2.31 & $2.07,1.98$ & \\
$\mathrm{G}^{271}$ & 8.65 & $4.02,3.96$ & & & \\
$\mathrm{D}^{272}$ & 8.13 & 4.68 & $2.75,2.73$ & & \\
$\mathrm{~T}^{273}$ & 8.21 & 4.30 & 4.25 & 1.20 & \\
$\mathrm{D}^{274}$ & 8.39 & 4.60 & $2.67,2.64$ & & & \\
$\mathrm{~L}^{275}$ & 8.04 & 4.25 & 1.47 & 1.40 & $0.87,0.78$ & $?$ \\
$\mathrm{Y}^{276}$ & 8.05 & 4.52 & $3.00,2.83$ & & $?$ \\
$\mathrm{D}^{277}$ & 8.15 & 4.54 & $2.57,2.53$ & & $?$ \\
$\mathrm{Y}^{278}$ & 7.82 & 4.47 & $2.88,2.81$ & & $?$ \\
$\mathrm{Y}^{279}$ & 8.09 & 4.72 & $3.00,2.77$ & & $?$ \\
$\mathrm{P}^{280}$ & & 4.32 & 2.25 & 1.94 & $?$ \\
$\mathrm{E}^{281}$ & 8.48 & 4.27 & 2.34 & $2.07,1.95$ & & $?$ \\
$\mathrm{E}^{282}$ & 8.44 & 4.31 & 2.28 & $2.06,1.93$ & & $?$ \\
$\mathrm{D}^{283}$ & 8.51 & 4.72 & $2.79,2.66$ & & \\
$\mathrm{~T}^{284}$ & 8.20 & 4.37 & 4.28 & 1.21 & \\
$\mathrm{E}^{285}$ & 8.44 & 4.36 & 2.30 & $2.11,1.97$ & & $?$ \\
$\mathrm{G}^{286}$ & 8.08 & 3.77 & & &
\end{tabular}

Table I: ${ }^{1} \mathrm{H}$ Resonance Assignments (ppm) of the GpIb $\alpha$ peptide (No Phosphorylation) bound to IIa at $17^{\circ} \mathrm{C}$.

\begin{tabular}{ccccccc} 
Residue & $\mathrm{NH}$ & $\mathrm{C}^{\alpha} \mathrm{H}$ & $\mathrm{C}^{\beta} \mathrm{H}$ & $\mathrm{C}^{\gamma} \mathrm{H}$ & $\mathrm{C}^{\delta} \mathrm{H}$ & $\mathrm{C}^{\varepsilon} \mathrm{H}$ \\
\hline $\mathrm{D}^{269}$ & $?$ & $?$ & $?$ & & & \\
$\mathrm{E}^{270}$ & 8.94 & 4.28 & 2.31 & $2.07,1.98$ & & \\
$\mathrm{G}^{271}$ & 8.64 & $4.04,3.96$ & & & \\
$\mathrm{D}^{272}$ & 8.13 & 4.68 & $2.75,2.73$ & & & \\
$\mathrm{~T}^{273}$ & 8.19 & 4.30 & 4.25 & 1.20 & & \\
$\mathrm{D}^{274}$ & 8.39 & 4.60 & $2.67,2.64$ & & & \\
$\mathrm{~L}^{275}$ & 8.01 & 4.25 & 1.47 & 1.40 & $0.87,0.78$ & \\
$\mathrm{Y}^{276}$ & 8.01 & 4.52 & $3.00,2.83$ & & & \\
$\mathrm{D}^{277}$ & 8.15 & 4.54 & $2.57,2.53$ & & 7.04 & 6.77 \\
$\mathrm{Y}^{278}$ & 7.82 & 4.47 & $2.88,2.81$ & & \\
$\mathrm{Y}^{279}$ & 8.02 & 4.72 & $3.00,2.77$ & & \\
$\mathrm{P}^{280}$ & & 4.32 & 2.25 & 1.94 & $3.48,3.43$ & \\
$\mathrm{E}^{281}$ & 8.47 & 4.27 & 2.34 & $2.07,1.95$ & & \\
$\mathrm{E}^{282}$ & 8.42 & 4.31 & 2.28 & $2.06,1.93$ & & \\
$\mathrm{D}^{283}$ & 8.48 & 4.72 & $2.79,2.66$ & & & \\
$\mathrm{~T}^{284}$ & 8.17 & 4.37 & 4.28 & 1.21 & & \\
$\mathrm{E}^{285}$ & 8.38 & 4.36 & 2.30 & $2.11,1.97$ & & \\
$\mathrm{G}^{286}$ & 8.07 & 3.77 & & & &
\end{tabular}


APPENDIX B INTER-RESIDUE NOE TABLE

Table J: Inter-residue NOEs for the $\gamma^{\prime}$ peptide $\left(\mathrm{Y}_{\mathrm{P}}{ }^{418} \mathrm{Y}_{\mathrm{P}}{ }^{422}\right)$ bound to IIa at a mixing time of $400 \mathrm{~ms}+15^{\circ} \mathrm{C}$.

\begin{tabular}{|c|c|c|c|c|c|c|c|}
\hline \multirow{2}{*}{\multicolumn{2}{|c|}{ Residue Proton }} & \multicolumn{6}{|c|}{ Inter-Residue NOE } \\
\hline & & $\mathrm{NH}$ & $\mathrm{C}^{\alpha} \mathrm{H}$ & $\mathrm{C}^{\beta} \mathrm{H}$ & $\mathrm{C}^{\gamma} \mathrm{H}$ & $\mathrm{C}^{\delta} \mathrm{H}$ & $\mathrm{C}^{\varepsilon} \mathrm{H}$ \\
\hline $\mathrm{P}^{410}$ & $\mathrm{C}^{\alpha} \mathrm{H}$ & $\mathrm{E}^{411}$ & & & & & \\
\hline \multirow{3}{*}{$E^{411}$} & $\mathrm{NH}$ & & $\mathrm{P}^{410}$ & & & & \\
\hline & $\mathrm{C}^{\alpha} \mathrm{H}$ & $\mathrm{H}^{412}$ & & & & & \\
\hline & $\mathrm{C}^{\beta} \mathrm{H}$ & $\mathrm{H}^{412}$ & & & & & \\
\hline \multirow{4}{*}{$\mathrm{H}^{412}$} & $\mathrm{NH}$ & & $E^{411} P^{413}$ & $\mathrm{E}^{411}$ & & $\mathrm{P}^{413}$ & \\
\hline & $\mathrm{C}^{\alpha} \mathrm{H}$ & & & & & $\mathrm{P}^{413}$ & \\
\hline & $\mathrm{C}^{\beta} \mathrm{H}$ & $A^{414}$ & & & & $\mathrm{P}^{413}$ & \\
\hline & $\mathrm{C}^{\delta} \mathrm{H}$ & & & & & $\mathrm{P}^{413}$ & \\
\hline \multirow{3}{*}{$\mathrm{P}^{413}$} & $\mathrm{C}^{\alpha} \mathrm{H}$ & $\mathrm{H}^{412} \mathrm{~A}^{414} \mathrm{E}^{415}$ & & $\mathrm{~A}^{414}$ & & & \\
\hline & $\mathrm{C}^{\gamma} \mathrm{H}$ & $\mathrm{A}^{414}$ & & & & & \\
\hline & $\mathrm{C}^{\delta} \mathrm{H}$ & $\mathrm{H}^{412} \mathrm{~A}^{414}$ & $\mathrm{H}^{412}$ & $\mathrm{H}^{412}$ & & $\mathrm{H}^{412}$ & \\
\hline \multirow{3}{*}{$\mathrm{A}^{414}$} & $\mathrm{NH}$ & & $\mathrm{P}^{413}$ & $\mathrm{H}^{412} \mathrm{E}^{415}$ & $P^{413} E^{415}$ & $\mathrm{P}^{413}$ & \\
\hline & $\mathrm{C}^{\alpha} \mathrm{H}$ & $E^{415}$ & & & & & \\
\hline & $\mathrm{C}^{\beta} \mathrm{H}$ & $E^{415}$ & $\mathrm{P}^{413}$ & $E^{415}$ & $E^{415}$ & & \\
\hline \multirow{3}{*}{$E^{415}$} & $\mathrm{NH}$ & $\mathrm{T}^{416}$ & $\mathrm{P}^{413} \mathrm{~A}^{414}$ & $\mathrm{~A}^{414}$ & & & \\
\hline & $\mathrm{C}^{\beta} \mathrm{H}$ & $A^{414} T^{416}$ & & $A^{414}$ & $T^{416}$ & & \\
\hline & $\mathrm{C}^{\gamma} \mathrm{H}$ & $A^{414} T^{416}$ & & $A^{414}$ & $T^{416}$ & & \\
\hline \multirow{4}{*}{$\mathrm{T}^{416}$} & $\mathrm{NH}$ & $\mathrm{E}^{415} \mathrm{E}^{417}$ & & $\mathrm{E}^{415}$ & $\mathrm{E}^{415}$ & & \\
\hline & $\mathrm{C}^{\alpha} \mathrm{H}$ & $E^{417}$ & & & & $\mathrm{Y}_{\mathrm{P}}^{418}$ & \\
\hline & $\mathrm{C}^{\beta} \mathrm{H}$ & $\mathrm{E}^{417}$ & & & & & \\
\hline & $\mathrm{C}^{\gamma} \mathrm{H}$ & $E^{417} Y_{P}^{418}$ & & $E^{415}$ & $E^{415}$ & $\mathrm{Y}_{\mathrm{P}}^{418}$ & \\
\hline \multirow{4}{*}{$E^{417}$} & $\mathrm{NH}$ & $\mathrm{T}^{416} \mathrm{Y}_{\mathrm{P}}^{418}$ & $\mathrm{~T}^{416}$ & $\mathrm{~T}^{416}$ & $\mathrm{~T}^{416}$ & & \\
\hline & $\mathrm{C}^{\alpha} \mathrm{H}$ & $\mathrm{Y}_{\mathrm{P}}^{418}$ & & $\mathrm{Y}_{\mathrm{P}}^{418}$ & & $\mathrm{Y}_{\mathrm{P}}^{418}$ & \\
\hline & $\mathrm{C}^{\beta} \mathrm{H}$ & $\mathrm{Y}_{\mathrm{P}}^{418}$ & & & & $\mathrm{Y}_{\mathrm{P}}^{418}$ & \\
\hline & $\mathrm{C}^{\gamma} \mathrm{H}$ & $\mathrm{Y}_{\mathrm{P}}^{418}$ & & & & $\mathrm{Y}_{\mathrm{P}}^{418}$ & \\
\hline \multirow{3}{*}{$\mathrm{Y}_{\mathrm{P}}^{418}$} & $\mathrm{NH}$ & $E^{417} D^{419}$ & $\mathrm{E}^{417}$ & $\mathrm{E}^{417}$ & $T^{416} E^{417}$ & & \\
\hline & $\mathrm{C}^{\beta} \mathrm{H}$ & $D^{419}$ & $E^{417}$ & & & & \\
\hline & $\mathrm{C}^{\delta} \mathrm{H}$ & $\mathrm{D}^{419}$ & $T^{416} E^{417}$ & $E^{417} D^{419}$ & $T^{416} E^{417}$ & $\mathrm{~L}^{421}$ & \\
\hline $\mathrm{D}^{419}$ & $\begin{array}{l}\mathrm{NH} \\
\mathrm{C}^{\beta} \mathrm{H}\end{array}$ & $\begin{array}{l}Y_{P}^{418} \\
S^{420}\end{array}$ & & $\mathrm{Y}_{\mathrm{P}}^{418}$ & & $\begin{array}{l}Y_{\mathrm{P}}^{418} \\
\mathrm{Y}_{\mathrm{P}}{ }^{418}\end{array}$ & \\
\hline \multirow{3}{*}{$S^{420}$} & $\mathrm{NH}$ & & & $\mathrm{D}^{419}$ & & & \\
\hline & $\mathrm{C}^{\alpha} \mathrm{H}$ & $\mathrm{L}^{421}$ & & $\mathrm{~L}^{421} \mathrm{Y}_{\mathrm{P}}^{422}$ & & $\mathrm{~L}^{421} \mathrm{Y}_{\mathrm{P}}^{422}$ & $\mathrm{Y}_{\mathrm{P}}^{422}$ \\
\hline & $\mathrm{C}^{\beta} \mathrm{H}$ & $\mathrm{L}^{421}$ & & & & $\mathrm{Y}_{\mathrm{P}}^{422}$ & $Y_{P}^{422}$ \\
\hline \multirow{5}{*}{$\mathrm{L}^{421}$} & $\mathrm{NH}$ & & $S^{420}$ & $\mathrm{~S}^{420} \mathrm{Y}_{\mathrm{P}}^{422}$ & & $\mathrm{Y}_{\mathrm{P}}^{422}$ & $Y_{P}^{422}$ \\
\hline & $\mathrm{C}^{\alpha} \mathrm{H}$ & $\mathrm{Y}_{\mathrm{P}}^{422}$ & & & & $Y_{P}^{422}$ & $Y_{P}^{422}$ \\
\hline & $\mathrm{C}^{\beta} \mathrm{H}$ & & $S^{420}$ & & & $\mathrm{Y}_{\mathrm{P}}^{422}$ & $Y_{P}^{422}$ \\
\hline & $\mathrm{C}^{\gamma} \mathrm{H}$ & & & & & $\mathrm{Y}_{\mathrm{P}}^{422}$ & $Y_{P}^{422}$ \\
\hline & $\mathrm{C}^{\delta} \mathrm{H}$ & & $S^{420}$ & & & $\mathrm{Y}_{\mathrm{P}}^{418} \mathrm{Y}_{\mathrm{P}}^{422}$ & $\mathrm{Y}_{\mathrm{P}}^{422}$ \\
\hline \multirow{4}{*}{$\mathrm{Y}_{\mathrm{P}}^{422}$} & $\mathrm{NH}$ & & $\mathrm{L}^{421}$ & & & & \\
\hline & $\mathrm{C}^{\beta} \mathrm{H}$ & $\mathrm{L}^{421} \mathrm{D}^{425}$ & $\begin{array}{c}\mathrm{S}^{420} \mathrm{P}^{423} \\
\mathrm{E}^{424}\end{array}$ & & $\mathrm{P}^{423}$ & $\mathrm{P}^{423}$ & \\
\hline & $\mathrm{C}^{\delta} \mathrm{H}$ & $\mathrm{L}^{421}$ & $\begin{array}{l}S^{420} L^{421} \\
P^{423} D^{425}\end{array}$ & $\begin{array}{l}S^{420} L^{421} \\
E^{424}\end{array}$ & $\begin{array}{l}\mathrm{L}^{421} \mathrm{P}^{423} \\
\mathrm{E}^{424}\end{array}$ & $\mathrm{~L}^{421} \mathrm{P}^{423}$ & \\
\hline & $\mathrm{C}^{\varepsilon} \mathrm{H}$ & $\mathrm{L}^{421}$ & $\begin{array}{l}\mathrm{S}^{420} \mathrm{~L}^{421} \\
\mathrm{P}^{423} \mathrm{D}^{425}\end{array}$ & $\mathrm{~S}^{420} \mathrm{~L}^{421}$ & $\mathrm{~L}^{421}$ & $\mathrm{~L}^{421} \mathrm{P}^{423}$ & \\
\hline
\end{tabular}


Table J: Inter-residue NOEs for the $\gamma^{\prime}$ peptide $\left(\mathrm{Y}_{\mathrm{P}}^{418} \mathrm{Y}_{\mathrm{P}}^{422}\right)$ bound to IIa at a mixing time of $400 \mathrm{~ms}+15^{\circ} \mathrm{C}$ (continued).

\begin{tabular}{|c|c|c|c|c|c|c|c|}
\hline \multicolumn{2}{|c|}{ Residue Proton } & NH & $\mathrm{C}^{\alpha} \mathrm{H}$ & $\begin{array}{c}\text { Inter-Residue } \mathrm{N} \\
\qquad \mathrm{C}^{\beta} \mathrm{H}\end{array}$ & $\begin{array}{l}\mathrm{C}^{\gamma} \\
\mathrm{H}\end{array}$ & $\mathrm{C}^{\delta} \mathrm{H}$ & $\mathrm{C}^{\varepsilon} \mathrm{H}$ \\
\hline \multirow{4}{*}{$\mathrm{P}^{423}$} & $\mathrm{C}^{\alpha} \mathrm{H}$ & $\mathrm{E}^{424} \mathrm{D}^{425}$ & & $\mathrm{Y}_{\mathrm{P}}^{422} \mathrm{E}^{424}$ & & $\mathrm{Y}_{\mathrm{P}}^{422}$ & $\mathrm{Y}_{\mathrm{P}}^{422}$ \\
\hline & $\mathrm{C}^{\beta} \mathrm{H}$ & $E^{424} D^{425}$ & & & & & \\
\hline & $\mathrm{C}^{\gamma} \mathrm{H}$ & & & $Y_{P}^{422}$ & & $Y_{P}^{422}$ & \\
\hline & $\mathrm{C}^{\delta} \mathrm{H}$ & & & $Y_{P}^{422}$ & & $\mathrm{Y}_{\mathrm{P}}^{422}$ & $\mathrm{Y}_{\mathrm{P}}^{422}$ \\
\hline \multirow{4}{*}{$E^{424}$} & $\mathrm{NH}$ & $\mathrm{D}^{425}$ & $\mathrm{P}^{423}$ & $\mathrm{P}^{423}$ & & & \\
\hline & $\mathrm{C}^{\alpha} \mathrm{H}$ & $D^{425}$ & & $\mathrm{Y}_{\mathrm{P}}^{422} \mathrm{D}^{425} \mathrm{~L}^{427}$ & $\mathrm{~L}_{7}^{42}$ & $\mathrm{~L}^{427}$ & \\
\hline & $\mathrm{C}^{\beta} \mathrm{H}$ & $D^{425}$ & $\mathrm{P}^{423}$ & & & $Y_{\mathrm{P}}^{422}$ & \\
\hline & $\mathrm{C}^{\gamma} \mathrm{H}$ & $D^{425}$ & & & & $\mathrm{Y}_{\mathrm{P}}^{422}$ & \\
\hline \multirow{3}{*}{$D^{425}$} & $\mathrm{NH}$ & $E^{424}$ & $\mathrm{P}^{423} \mathrm{E}^{424}$ & $\mathrm{Y}_{\mathrm{P}}^{422} \mathrm{P}^{423} \mathrm{E}^{424}$ & $\begin{array}{c}\mathrm{E}_{4}^{42} \\
\end{array}$ & & \\
\hline & $\mathrm{C}^{\alpha} \mathrm{H}$ & & & & & $\mathrm{Y}_{\mathrm{P}}^{422}$ & $\mathrm{Y}_{\mathrm{P}}^{422}$ \\
\hline & $\mathrm{C}^{\beta} \mathrm{H}$ & & $E^{424}$ & & & & \\
\hline \multirow{3}{*}{$D^{426}$} & $\mathrm{NH}$ & $\mathrm{L}^{427}$ & & & & & \\
\hline & $\mathrm{C}^{\alpha} \mathrm{H}$ & $L^{427}$ & & $\mathrm{~L}^{427}$ & $\mathrm{~L}_{7}^{42}$ & & \\
\hline & $\mathrm{C}^{\beta} \mathrm{H}$ & $\mathrm{L}^{427}$ & & & & & \\
\hline \multirow{4}{*}{$\mathrm{L}^{427}$} & $\mathrm{NH}$ & $\mathrm{D}^{426}$ & $\mathrm{D}^{426}$ & $D^{426}$ & & & \\
\hline & $\mathrm{C}^{\beta} \mathrm{H}$ & & $E^{424} D^{426}$ & & & & \\
\hline & $\mathrm{C}^{\gamma} \mathrm{H}$ & & $E^{424} D^{426}$ & & & & \\
\hline & $\mathrm{C}^{\delta} \mathrm{H}$ & & $E^{424}$ & & & & \\
\hline
\end{tabular}


Table K: Inter-residue NOEs for the $\gamma^{\prime}$ peptide $\left(\mathrm{Y}_{\mathrm{P}}^{418}\right)$ bound to IIa at a mixing time of $400 \mathrm{~ms}+15^{\circ} \mathrm{C}$.

\begin{tabular}{|c|c|c|c|c|c|c|c|}
\hline \multicolumn{2}{|c|}{ Residue Proton } & $\mathrm{NH}$ & \multicolumn{4}{|c|}{ Inter-Residue NOE } & $\mathrm{C}^{\varepsilon} \mathrm{H}$ \\
\hline $\mathrm{P}^{410}$ & $\mathrm{C}^{\alpha} \mathrm{H}$ & $\mathrm{E}^{411}$ & & & & & \\
\hline \multirow{3}{*}{$\mathrm{E}^{411}$} & $\mathrm{NH}$ & & $\mathrm{P}^{410}$ & & & & \\
\hline & $\mathrm{C}^{\alpha} \mathrm{H}$ & $\mathrm{H}^{412}$ & & & & & \\
\hline & $\mathrm{C}^{\beta} \mathrm{H}$ & & & & & & \\
\hline \multirow{4}{*}{$\mathrm{H}^{412}$} & $\mathrm{NH}$ & & $\mathrm{E}^{411} \mathrm{P}^{413}$ & $E^{41 \Gamma}$ & & $\mathrm{P}^{413}$ & \\
\hline & $\mathrm{C}^{\alpha} \mathrm{H}$ & & & & & $\mathrm{P}^{413}$ & \\
\hline & $\mathrm{C}^{\beta} \mathrm{H}$ & $A^{414}$ & & & & $\mathrm{P}^{413}$ & \\
\hline & $\mathrm{C}^{\delta} \mathrm{H}$ & & & & & $\mathrm{P}^{413}$ & \\
\hline \multirow{2}{*}{$\mathrm{P}^{413}$} & $\mathrm{C}^{\alpha} \mathrm{H}$ & $\mathrm{H}^{412} \mathrm{~A}^{414} \mathrm{E}^{415}$ & & $\mathrm{~A}^{414}$ & & & \\
\hline & $\mathrm{C}^{\delta} \mathrm{H}$ & $\mathrm{H}^{412} \mathrm{~A}^{414}$ & $\mathrm{H}^{412}$ & $\mathrm{H}^{412}$ & & $\mathrm{H}^{412}$ & \\
\hline \multirow{3}{*}{$A^{414}$} & $\mathrm{NH}$ & & $\mathrm{P}^{413}$ & $\mathrm{H}^{412}$ & & $\mathrm{P}^{413}$ & \\
\hline & $\mathrm{C}^{\alpha} \mathrm{H}$ & $E^{415}$ & & & & & \\
\hline & $\mathrm{C}^{\beta} \mathrm{H}$ & $E^{415}$ & $\mathrm{P}^{413}$ & & & & \\
\hline \multirow{3}{*}{$E^{415}$} & $\mathrm{NH}$ & $\mathrm{T}^{416}$ & $\mathrm{P}^{413} \mathrm{~A}^{414}$ & $\mathrm{~A}^{414}$ & $\mathrm{~T}^{416}$ & & \\
\hline & $\mathrm{C}^{\beta} \mathrm{H}$ & $\mathrm{T}^{416}$ & & & & & \\
\hline & $\mathrm{C}^{\gamma} \mathrm{H}$ & $\mathrm{T}^{416}$ & & & & & \\
\hline \multirow{4}{*}{$\mathrm{T}^{416}$} & $\mathrm{NH}$ & $E^{415} E^{417}$ & & $\mathrm{E}^{415}$ & $E^{415}$ & & \\
\hline & $\mathrm{C}^{\alpha} \mathrm{H}$ & $\mathrm{E}^{417}$ & & & & $\mathrm{Y}_{\mathrm{P}}^{418}$ & $\mathrm{Y}_{\mathrm{P}}^{418}$ \\
\hline & $\mathrm{C}^{\beta} \mathrm{H}$ & $E^{417}$ & & & & $Y_{P}^{418}$ & $Y_{P}^{418}$ \\
\hline & $\mathrm{C}^{\gamma} \mathrm{H}$ & $\mathrm{E}^{415} \mathrm{E}^{417} \mathrm{Y}_{\mathrm{P}}^{418}$ & & & & $\mathrm{Y}_{\mathrm{P}}^{418}$ & \\
\hline \multirow{4}{*}{$E^{417}$} & $\mathrm{NH}$ & $\mathrm{T}^{416} \mathrm{Y}_{\mathrm{P}}^{418}$ & $T^{416}$ & $\mathrm{~T}^{416}$ & $\mathrm{~T}^{416}$ & $\mathrm{Y}_{\mathrm{P}}^{418}$ & \\
\hline & $\mathrm{C}^{\alpha} \mathrm{H}$ & $\mathrm{Y}_{\mathrm{P}}^{418}$ & & $\mathrm{Y}_{\mathrm{P}}^{418}$ & & $\mathrm{Y}_{\mathrm{P}}^{418}$ & $Y_{P}^{418}$ \\
\hline & $\mathrm{C}^{\beta} \mathrm{H}$ & $\mathrm{Y}_{\mathrm{P}}^{418}$ & & & & $Y_{P}^{418}$ & \\
\hline & $\mathrm{C}^{\gamma} \mathrm{H}$ & $\mathrm{Y}_{\mathrm{P}}^{418}$ & & & & $\mathrm{Y}_{\mathrm{P}}^{418}$ & \\
\hline \multirow{4}{*}{$\mathrm{Y}_{\mathrm{P}}^{418}$} & $\mathrm{NH}$ & $\mathrm{E}^{417} \mathrm{D}^{419}$ & $\mathrm{E}^{417}$ & $\mathrm{E}^{417}$ & $T^{416} E^{417}$ & & \\
\hline & $\mathrm{C}^{\beta} \mathrm{H}$ & $D^{419} S^{420}$ & $E^{417}$ & & & & \\
\hline & $\mathrm{C}^{\delta} \mathrm{H}$ & $\mathrm{E}^{417} \mathrm{D}^{419} \mathrm{~L}^{421}$ & $T^{416} E^{417}$ & $\mathrm{~T}^{416} \mathrm{E}_{\mathrm{S}^{420}}^{417} \mathrm{D}^{419}$ & $\mathrm{~T}^{416} \mathrm{E}^{417}$ & $\mathrm{~L}^{421}$ & \\
\hline & $\mathrm{C}^{\varepsilon} \mathrm{H}$ & $\mathrm{D}^{419}$ & $T^{416} E^{417}$ & $\mathrm{~T}^{416} \mathrm{~S}^{420}$ & & & \\
\hline \multirow{3}{*}{$D^{419}$} & $\mathrm{NH}$ & $Y_{P}^{418} S^{420}$ & & $\mathrm{Y}_{\mathrm{P}}^{418}$ & & $\mathrm{Y}_{\mathrm{P}}^{418}$ & $\mathrm{Y}_{\mathrm{P}}^{418}$ \\
\hline & $\mathrm{C}^{\alpha} \mathrm{H}$ & $\mathrm{S}^{420}$ & & & & & \\
\hline & $\mathrm{C}^{\beta} \mathrm{H}$ & $S^{420}$ & & & & $\mathrm{Y}_{\mathrm{P}}^{418}$ & \\
\hline \multirow{3}{*}{$S^{420}$} & $\mathrm{NH}$ & $\mathrm{D}^{419}$ & $\mathrm{D}^{419}$ & $\mathrm{Y}_{\mathrm{P}}^{418} \mathrm{D}^{419}$ & & & \\
\hline & $\mathrm{C}^{\alpha} \mathrm{H}$ & & & $L^{421}$ & $\mathrm{~L}^{421}$ & $\mathrm{~L}^{421} \mathrm{Y}^{422}$ & $\mathrm{Y}^{422}$ \\
\hline & $\mathrm{C}^{\beta} \mathrm{H}$ & $\mathrm{L}^{421}$ & & & & $\mathrm{Y}_{\mathrm{P}}^{418} \mathrm{Y}^{422}$ & $\begin{array}{l}\mathrm{Y}_{\mathrm{P}}^{418} \\
\mathrm{Y}^{422}\end{array}$ \\
\hline \multirow{5}{*}{$\mathrm{L}^{421}$} & $\mathrm{NH}$ & & & $S^{420}$ & & $\mathrm{Y}_{\mathrm{P}}^{418} \mathrm{Y}^{422}$ & $\mathrm{Y}^{422}$ \\
\hline & $\mathrm{C}^{\alpha} \mathrm{H}$ & $\mathrm{Y}^{422}$ & & & & $Y^{422}$ & $\mathrm{Y}^{422}$ \\
\hline & $\mathrm{C}^{\beta} \mathrm{H}$ & & $S^{420} Y^{422}$ & & & $\mathrm{Y}^{422}$ & $\mathrm{Y}^{422}$ \\
\hline & $\mathrm{C}^{\gamma} \mathrm{H}$ & & $S^{420}$ & & & $\mathrm{Y}_{\mathrm{P}}^{418} \mathrm{Y}^{422}$ & $\mathrm{Y}^{422}$ \\
\hline & $\mathrm{C}^{\delta} \mathrm{H}$ & & $S^{420}$ & & & $\mathrm{Y}_{\mathrm{P}}^{418} \mathrm{Y}^{422}$ & $\mathrm{Y}^{422}$ \\
\hline \multirow{4}{*}{$\mathrm{Y}^{422}$} & $\mathrm{NH}$ & & $\mathrm{L}^{421}$ & & & $\mathrm{P}^{423}$ & \\
\hline & $\mathrm{C}^{\beta} \mathrm{H}$ & $E^{424} D^{425}$ & $\mathrm{~L}^{421} \mathrm{P}^{423}$ & & & $\mathrm{P}^{423}$ & \\
\hline & $\mathrm{C}^{\delta} \mathrm{H}$ & $\mathrm{L}^{421} \mathrm{E}^{424} \mathrm{D}^{425}$ & $\begin{array}{c}\mathrm{S}^{420} \mathrm{~L}^{421} \\
\mathrm{P}^{423} \mathrm{E}^{424} \mathrm{D}^{425}\end{array}$ & $\begin{array}{l}\mathrm{S}^{420} \mathrm{~L}^{421} \\
\mathrm{P}^{423} \mathrm{~F}^{424}\end{array}$ & $\mathrm{~L}^{421} \mathrm{P}^{423}$ & $\mathrm{~L}^{421} \mathrm{P}^{423}$ & \\
\hline & $\mathrm{C}^{\varepsilon} \mathrm{H}$ & $\mathrm{L}^{421} \mathrm{D}^{425}$ & $\mathrm{~S}^{420} \mathrm{~L}^{421} \mathrm{E}^{424}$ & $\mathrm{~S}^{420} \mathrm{~L}^{421}$ & $\mathrm{~L}^{421}$ & $\mathrm{~L}^{421} \mathrm{P}^{423}$ & \\
\hline
\end{tabular}


Table K: Inter-residue NOEs for the $\gamma^{\prime}$ peptide $\left(\mathrm{Y}_{\mathrm{P}}{ }^{418}\right)$ bound to IIa at a mixing time of $400 \mathrm{~ms}+15^{\circ} \mathrm{C}$ (continued).

\begin{tabular}{|c|c|c|c|c|c|c|c|}
\hline \multirow{2}{*}{\multicolumn{2}{|c|}{ Residue Proton }} & \multicolumn{6}{|c|}{ Inter-Residue NOE } \\
\hline & & $\mathrm{NH}$ & $\mathrm{C}^{\alpha} \mathrm{H}$ & $\mathrm{C}^{\beta} \mathrm{H}$ & $\mathrm{C}^{\gamma} \mathrm{H}$ & $\mathrm{C}^{\delta} \mathrm{H}$ & $\mathrm{C}^{\varepsilon} \mathrm{H}$ \\
\hline \multirow{4}{*}{$\mathrm{P}^{423}$} & $\mathrm{C}^{\alpha} \mathrm{H}$ & $\mathrm{E}^{424} \mathrm{D}^{425}$ & & $Y^{422} E^{424}$ & & $\mathrm{Y}^{422}$ & \\
\hline & $\mathrm{C}^{\beta} \mathrm{H}$ & $\mathrm{E}^{424} \mathrm{D}^{425}$ & & & & $\mathrm{Y}^{422}$ & \\
\hline & $\mathrm{C}^{\gamma} \mathrm{H}$ & & & & & $\mathrm{Y}^{422}$ & \\
\hline & $\mathrm{C}^{\delta} \mathrm{H}$ & $E^{424} Y^{422}$ & & $Y^{422}$ & & $\mathrm{Y}^{422}$ & $\mathrm{Y}^{422}$ \\
\hline \multirow{4}{*}{$\mathrm{E}^{424}$} & $\mathrm{NH}$ & $\mathrm{D}^{425}$ & $\mathrm{P}^{423}$ & $\mathrm{Y}^{422} \mathrm{P}^{423}$ & & $\mathrm{Y}^{422} \mathrm{P}^{423}$ & \\
\hline & $\mathrm{C}^{\alpha} \mathrm{H}$ & $\mathrm{D}^{425}$ & & $\mathrm{D}^{425} \mathrm{~L}^{427}$ & $\mathrm{~L}^{427}$ & $\mathrm{Y}^{422} \mathrm{~L}^{427}$ & \\
\hline & $\mathrm{C}^{\beta} \mathrm{H}$ & $\mathrm{D}^{425}$ & $\mathrm{P}^{423}$ & & & $\mathrm{Y}^{422}$ & \\
\hline & $\mathrm{C}^{\gamma} \mathrm{H}$ & $\mathrm{D}^{425}$ & & & & & \\
\hline \multirow{2}{*}{$\mathrm{D}^{425}$} & $\mathrm{NH}$ & $E^{424}$ & $\mathrm{P}^{423} \mathrm{E}^{424}$ & $\mathrm{Y}^{422} \mathrm{P}^{423} \mathrm{E}^{424}$ & $E^{424}$ & $\mathrm{Y}^{422}$ & $\mathrm{Y}^{422}$ \\
\hline & $\mathrm{C}^{\beta} \mathrm{H}$ & $\mathrm{L}^{427}$ & $E^{424}$ & & & $\mathrm{Y}^{422}$ & \\
\hline \multirow{3}{*}{$\mathrm{D}^{426}$} & $\mathrm{NH}$ & $\mathrm{L}^{427}$ & & & & & \\
\hline & $\mathrm{C}^{\alpha} \mathrm{H}$ & $\mathrm{L}^{427}$ & & $\mathrm{~L}^{427}$ & $\mathrm{~L}^{427}$ & & \\
\hline & $\mathrm{C}^{\beta} \mathrm{H}$ & $\mathrm{L}^{427}$ & & & & & \\
\hline \multirow{4}{*}{$\mathrm{L}^{427}$} & $\mathrm{NH}$ & $\mathrm{D}^{426}$ & $\mathrm{D}^{426}$ & $\mathrm{D}^{425} \mathrm{D}^{426}$ & & & \\
\hline & $\mathrm{C}^{\beta} \mathrm{H}$ & & $\mathrm{E}^{424} \mathrm{D}^{426}$ & & & & \\
\hline & $\mathrm{C}^{\gamma} \mathrm{H}$ & & $\mathrm{E}^{424} \mathrm{D}^{426}$ & & & & \\
\hline & $\mathrm{C}^{\delta} \mathrm{H}$ & & $E^{424}$ & & & & \\
\hline
\end{tabular}


Table L: Inter-residue NOEs for the $\gamma^{\prime}$ peptide $\left(\mathrm{Y}_{\mathrm{P}}{ }^{422}\right)$ bound to IIa at a mixing time of $400 \mathrm{~ms}+15^{\circ} \mathrm{C}$.

\begin{tabular}{|c|c|c|c|c|c|c|c|}
\hline \multirow{2}{*}{\multicolumn{2}{|c|}{ Residue Proton }} & \multicolumn{6}{|c|}{ Inter-Residue NOE } \\
\hline & & $\mathrm{NH}$ & $\mathrm{C}^{\alpha} \mathrm{H}$ & $\mathrm{C}^{\beta} \mathrm{H}$ & $\mathrm{C}^{\gamma} \mathrm{H}$ & $\mathrm{C}^{\delta} \mathrm{H}$ & $\mathrm{C}^{\varepsilon} \mathrm{H}$ \\
\hline $\mathrm{P}^{410}$ & $\mathrm{C}^{\alpha} \mathrm{H}$ & $E^{411}$ & & & & & \\
\hline \multirow{3}{*}{$E^{411}$} & $\mathrm{NH}$ & & $\mathrm{P}^{410}$ & & & & \\
\hline & $\mathrm{C}^{\alpha} \mathrm{H}$ & $\mathrm{H}^{412}$ & & & & & \\
\hline & $\mathrm{C}^{\beta} \mathrm{H}$ & $\mathrm{H}^{412}$ & & & & & \\
\hline \multirow{4}{*}{$\mathrm{H}^{412}$} & $\mathrm{NH}$ & & $E^{411} P^{413}$ & $\mathrm{E}^{411}$ & & $\mathrm{P}^{413}$ & \\
\hline & $\mathrm{C}^{\alpha} \mathrm{H}$ & & & & & $\mathrm{P}^{413}$ & \\
\hline & $\mathrm{C}^{\beta} \mathrm{H}$ & $A^{414}$ & & & & $\mathrm{P}^{413}$ & \\
\hline & $\mathrm{C}^{\delta} \mathrm{H}$ & & & & & $\mathrm{P}^{413}$ & \\
\hline \multirow{3}{*}{$\mathrm{P}^{413}$} & $\mathrm{C}^{\alpha} \mathrm{H}$ & $\mathrm{H}^{412} \mathrm{~A}^{414} \mathrm{E}^{415}$ & & $A^{414}$ & & & \\
\hline & $\mathrm{C}^{\gamma} \mathrm{H}$ & $\mathrm{A}^{414}$ & & & & & \\
\hline & $\mathrm{C}^{\delta} \mathrm{H}$ & $\mathrm{H}^{412} \mathrm{~A}^{414}$ & $\mathrm{H}^{412}$ & $\mathrm{H}^{412}$ & & $\mathrm{H}^{412}$ & \\
\hline \multirow{3}{*}{$\mathrm{A}^{414}$} & $\mathrm{NH}$ & & $\mathrm{P}^{413}$ & $\mathrm{H}^{412} \mathrm{E}^{415}$ & $\mathrm{P}^{413} \mathrm{E}^{415}$ & $\mathrm{P}^{413}$ & \\
\hline & $\mathrm{C}^{\alpha} \mathrm{H}$ & $\mathrm{E}^{415}$ & & & & & \\
\hline & $\mathrm{C}^{\beta} \mathrm{H}$ & $E^{415}$ & $\mathrm{P}^{413}$ & & $E^{415}$ & & \\
\hline \multirow{3}{*}{$E^{415}$} & $\mathrm{NH}$ & $\mathrm{T}^{416}$ & $\mathrm{P}^{413} \mathrm{~A}^{414}$ & $\mathrm{~A}^{414}$ & $\mathrm{~T}^{416}$ & & \\
\hline & $\mathrm{C}^{\beta} \mathrm{H}$ & $A^{414} T^{416}$ & & & $\mathrm{~T}^{416}$ & & \\
\hline & $\mathrm{C}^{\gamma} \mathrm{H}$ & $\mathrm{A}^{414} \mathrm{~T}^{416}$ & & $\mathrm{~A}^{414}$ & $T^{416}$ & & \\
\hline \multirow{4}{*}{$\mathrm{T}^{416}$} & $\mathrm{NH}$ & $E^{415} E^{417}$ & & $\mathrm{E}^{415}$ & $\mathrm{E}^{415}$ & & \\
\hline & $\mathrm{C}^{\alpha} \mathrm{H}$ & $E^{417}$ & & & & $\mathrm{Y}^{418}$ & $\mathrm{Y}^{418}$ \\
\hline & $\mathrm{C}^{\beta} \mathrm{H}$ & $\mathrm{E}^{417}$ & & & & & \\
\hline & $\mathrm{C}^{\gamma} \mathrm{H}$ & $E^{415} E^{417}$ & $E^{417}$ & $E^{415}$ & $E^{415}$ & $\mathrm{Y}^{418}$ & $\mathrm{Y}^{418}$ \\
\hline \multirow{4}{*}{$E^{417}$} & $\mathrm{NH}$ & $\mathrm{T}^{416}$ & $T^{416}$ & $\mathrm{~T}^{416}$ & $\mathrm{~T}^{416}$ & $\mathrm{Y}^{418}$ & \\
\hline & $\mathrm{C}^{\alpha} \mathrm{H}$ & $\mathrm{Y}^{418}$ & & & $\mathrm{~T}^{416}$ & $\mathrm{Y}^{418}$ & $\mathrm{Y}^{418}$ \\
\hline & $\mathrm{C}^{\beta} \mathrm{H}$ & $\mathrm{Y}^{418}$ & & & & $\mathrm{Y}^{418}$ & $\mathrm{Y}^{418}$ \\
\hline & $\mathrm{C}^{\gamma} \mathrm{H}$ & $\mathrm{Y}^{418}$ & & & & $\mathrm{Y}^{418}$ & $\mathrm{Y}^{418}$ \\
\hline \multirow{4}{*}{$\mathrm{Y}^{418}$} & $\mathrm{NH}$ & $\mathrm{D}^{419}$ & $\mathrm{E}^{417}$ & $\mathrm{E}^{417}$ & $\mathrm{E}^{417}$ & & \\
\hline & $\mathrm{C}^{\beta} \mathrm{H}$ & $\mathrm{D}^{419}$ & & & & & \\
\hline & $\mathrm{C}^{\delta} \mathrm{H}$ & $E^{417} D^{419}$ & $T^{416} E^{417}$ & $E^{417} D^{419}$ & $\mathrm{~T}^{416} \mathrm{E}^{417}$ & $\mathrm{~L}^{421}$ & \\
\hline & $\mathrm{C}^{\varepsilon} \mathrm{H}$ & $\mathrm{D}^{419}$ & $\mathrm{~T}^{416} \mathrm{E}^{417}$ & $E^{417}$ & $\mathrm{~T}^{416} \mathrm{E}^{417}$ & $\mathrm{~L}^{421}$ & \\
\hline \multirow{3}{*}{$\mathrm{D}^{419}$} & $\mathrm{NH}$ & $\mathrm{Y}^{418}$ & & $\mathrm{Y}^{418}$ & & $\mathrm{Y}^{418}$ & $\mathrm{Y}^{418}$ \\
\hline & $\mathrm{C}^{\alpha} \mathrm{H}$ & $S^{420}$ & & & & $v^{418}$ & \\
\hline & $\mathrm{C}^{\beta} \mathrm{H}$ & $S^{420}$ & & & & $\mathrm{Y}^{418}$ & \\
\hline \multirow{3}{*}{$S^{420}$} & $\mathrm{NH}$ & & $\mathrm{D}^{419}$ & $\mathrm{D}^{419}$ & & & \\
\hline & $\mathrm{C}^{\alpha} \mathrm{H}$ & & & $\mathrm{L}^{421}$ & $\mathrm{~L}^{421}$ & $\mathrm{~L}^{421} \mathrm{Y}_{\mathrm{P}}^{422}$ & \\
\hline & $\mathrm{C}^{\beta} \mathrm{H}$ & $\mathrm{L}^{421}$ & & & & $\mathrm{Y}_{\mathrm{P}}^{422}$ & $Y_{P}^{422}$ \\
\hline \multirow{5}{*}{$\mathrm{L}^{421}$} & $\mathrm{NH}$ & & & $S^{420}$ & & $Y_{P}^{422}$ & $\mathrm{Y}_{\mathrm{P}}^{422}$ \\
\hline & $\mathrm{C}^{\alpha} \mathrm{H}$ & & & & & $Y_{P}^{422}$ & \\
\hline & $\mathrm{C}^{\beta} \mathrm{H}$ & & $S^{420}$ & & & $Y_{P}^{422}$ & $Y_{P}^{422}$ \\
\hline & $\mathrm{C}^{\gamma} \mathrm{H}$ & & $S^{420}$ & & & $\mathrm{Y}_{\mathrm{P}}^{422}$ & $\mathrm{Y}_{\mathrm{P}}^{422}$ \\
\hline & $\mathrm{C}^{\delta} \mathrm{H}$ & & $S^{420}$ & & & $\mathrm{Y}^{418} \mathrm{Y}_{\mathrm{P}}^{422}$ & $\begin{array}{l}\mathrm{Y}^{418} \\
\mathrm{Y}_{\mathrm{P}}^{422}\end{array}$ \\
\hline \multirow{3}{*}{$\mathrm{Y}_{\mathrm{P}}^{422}$} & $\mathrm{C}^{\beta} \mathrm{H}$ & $\mathrm{E}^{424} \mathrm{D}^{425}$ & $\mathrm{P}^{423}$ & & $\mathrm{P}^{423}$ & $\mathrm{P}^{423}$ & \\
\hline & $\mathrm{C}^{\delta} \mathrm{H}$ & $\mathrm{L}^{421} \mathrm{E}^{424} \mathrm{D}^{425}$ & $\begin{array}{l}\mathrm{S}^{420} \mathrm{~L}^{421} \\
\mathrm{P}^{423} \mathrm{D}^{425}\end{array}$ & $\begin{array}{c}S^{420} L^{421} \\
E^{424}\end{array}$ & $\begin{array}{c}\mathrm{L}^{421} \mathrm{P}^{423} \\
\mathrm{E}^{424}\end{array}$ & $\mathrm{~L}^{421} \mathrm{P}^{423}$ & \\
\hline & $\mathrm{C}^{\varepsilon} \mathrm{H}$ & $\mathrm{L}^{421} \mathrm{E}^{424} \mathrm{D}^{425}$ & $\mathrm{P}^{423}$ & $\mathrm{~S}^{420} \mathrm{~L}^{421}$ & $\mathrm{~L}^{421}$ & $\mathrm{~L}^{421} \mathrm{P}^{423}$ & \\
\hline
\end{tabular}


Table L: Inter-residue NOEs for the $\gamma^{\prime}$ peptide $\left(\mathrm{Y}_{\mathrm{P}}^{422}\right)$ bound to IIa at a mixing time of $400 \mathrm{~ms}+15^{\circ} \mathrm{C}$ (continued).

\begin{tabular}{|c|c|c|c|c|c|c|c|}
\hline \multirow{2}{*}{\multicolumn{2}{|c|}{ Residue Proton }} & \multicolumn{6}{|c|}{ Inter-Residue NOE } \\
\hline & & $\mathrm{NH}$ & $\mathrm{C}^{\alpha} \mathrm{H}$ & $\mathrm{C}^{\beta} \mathrm{H}$ & $\mathrm{C}^{\gamma} \mathrm{H}$ & $\mathrm{C}^{\delta} \mathrm{H}$ & $\mathrm{C}^{\varepsilon} \mathrm{H}$ \\
\hline \multirow{4}{*}{$\mathrm{P}^{423}$} & $\mathrm{C}^{\alpha} \mathrm{H}$ & $\mathrm{E}^{424} \mathrm{D}^{425}$ & & $Y_{P}^{422} E^{424}$ & & $\mathrm{Y}_{\mathrm{P}}^{422}$ & $\mathrm{Y}_{\mathrm{P}}^{422}$ \\
\hline & $\mathrm{C}^{\beta} \mathrm{H}$ & $E^{424} D^{425}$ & & & & & \\
\hline & $\mathrm{C}^{\gamma} \mathrm{H}$ & $E^{424}$ & & $\mathrm{Y}_{\mathrm{P}}^{422}$ & & $\mathrm{Y}_{\mathrm{P}}^{422}$ & \\
\hline & $\mathrm{C}^{\delta} \mathrm{H}$ & & & $Y_{P}^{422}$ & & $Y_{P}^{422}$ & $Y_{P}^{422}$ \\
\hline \multirow{4}{*}{$E^{424}$} & $\mathrm{NH}$ & $D^{425}$ & $\mathrm{P}^{423}$ & $\mathrm{Y}_{\mathrm{P}}^{422} \mathrm{P}^{423}$ & $\mathrm{P}^{423}$ & $\mathrm{Y}_{\mathrm{P}}^{422}$ & $\mathrm{Y}_{\mathrm{P}}^{422}$ \\
\hline & $\mathrm{C}^{\alpha} \mathrm{H}$ & $D^{425}$ & & $\mathrm{D}^{425} \mathrm{~L}^{427}$ & $\mathrm{~L}^{427}$ & $\mathrm{~L}^{427}$ & \\
\hline & $\mathrm{C}^{\beta} \mathrm{H}$ & $D^{425}$ & $\mathrm{P}^{423}$ & & & $Y_{P}^{422}$ & \\
\hline & $\mathrm{C}^{\gamma} \mathrm{H}$ & $\mathrm{D}^{425}$ & & & & $\mathrm{Y}_{\mathrm{P}}^{422}$ & \\
\hline \multirow{3}{*}{$D^{425}$} & $\mathrm{NH}$ & $E^{424}$ & $\mathrm{P}^{423} \mathrm{E}^{424}$ & $\mathrm{Y}_{\mathrm{P}}^{422} \mathrm{P}^{423} \mathrm{E}^{424}$ & $E^{424}$ & $Y_{P}^{422}$ & $Y_{P}^{422}$ \\
\hline & $\mathrm{C}^{\alpha} \mathrm{H}$ & & & & & $Y_{P}^{422}$ & \\
\hline & $\mathrm{C}^{\beta} \mathrm{H}$ & $\mathrm{L}^{427}$ & $E^{424}$ & & & & \\
\hline \multirow{3}{*}{$D^{426}$} & $\mathrm{NH}$ & $\mathrm{L}^{427}$ & $\mathrm{D}^{425}$ & $\mathrm{D}^{425}$ & & & $\mathrm{Y}_{\mathrm{P}}^{422}$ \\
\hline & $\mathrm{C}^{\alpha} \mathrm{H}$ & $\mathrm{L}^{427}$ & & $\mathrm{~L}^{427}$ & $\mathrm{~L}^{427}$ & & \\
\hline & $\mathrm{C}^{\beta} \mathrm{H}$ & $\mathrm{L}^{427}$ & & & & & \\
\hline \multirow{4}{*}{$\mathrm{L}^{427}$} & $\mathrm{NH}$ & $\mathrm{D}^{426}$ & $\mathrm{D}^{426}$ & $\mathrm{D}^{425} \mathrm{D}^{426}$ & & & \\
\hline & $\mathrm{C}^{\beta} \mathrm{H}$ & & $E^{424} D^{426}$ & & & & \\
\hline & $\mathrm{C}^{\gamma} \mathrm{H}$ & & $E^{424} D^{426}$ & & & & \\
\hline & $\mathrm{C}^{\delta} \mathrm{H}$ & & $E^{424}$ & & & & \\
\hline
\end{tabular}

Table M: Inter-residue NOEs for 1). free $\gamma^{\prime}$ peptides and 2.) unphosphorylated $\gamma^{\prime}$ peptide in the presence of IIa at a mixing time of $400 \mathrm{~ms}+17^{\circ} \mathrm{C}$. There are not many significant differences in NOE assignments across this series of NMR experiments.

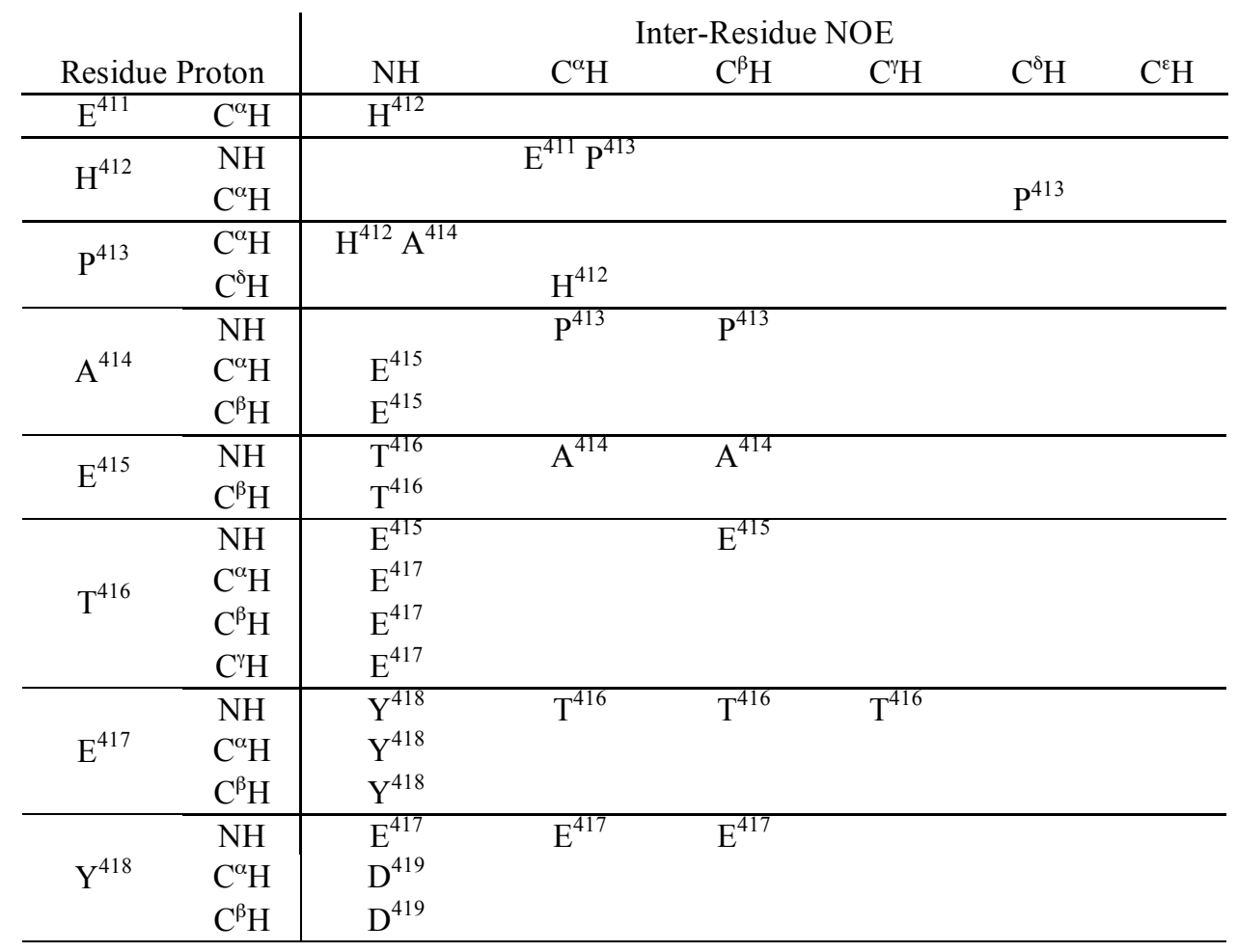


Table M: Inter-residue NOEs for 1). free $\gamma^{\prime}$ peptides and 2.) unphosphorylated $\gamma^{\prime}$ peptide in the presence of IIa at a mixing time of $400 \mathrm{~ms}+17^{\circ} \mathrm{C}$. There are not many significant differences in NOE assignments across this series of NMR experiments (continued).

\begin{tabular}{|c|c|c|c|c|c|c|c|}
\hline \multirow{2}{*}{\multicolumn{2}{|c|}{ Residue Proton }} & \multicolumn{6}{|c|}{ Inter-Residue NOE } \\
\hline & & $\mathrm{NH}$ & $\mathrm{C}^{\alpha} \mathrm{H}$ & $\mathrm{C}^{\beta} \mathrm{H}$ & $\mathrm{C}^{\gamma} \mathrm{H}$ & $\mathrm{C}^{\delta} \mathrm{H}$ & $\mathrm{C}^{\varepsilon} \mathrm{H}$ \\
\hline \multirow{3}{*}{$\mathrm{D}^{419}$} & $\mathrm{NH}$ & & $\mathrm{Y}^{418}$ & $\mathrm{Y}^{418}$ & & & \\
\hline & $\mathrm{C}^{\alpha} \mathrm{H}$ & $S^{420}$ & & & & & \\
\hline & $\mathrm{C}^{\beta} \mathrm{H}$ & $S^{420}$ & & & & & \\
\hline \multirow{3}{*}{$S^{420}$} & $\mathrm{NH}$ & & $\mathrm{D}^{419}$ & $\mathrm{D}^{419}$ & & & \\
\hline & $\mathrm{C}^{\alpha} \mathrm{H}$ & $\mathrm{L}^{421}$ & & & & & \\
\hline & $\mathrm{C}^{\beta} \mathrm{H}$ & $\mathrm{L}^{421}$ & & & & & \\
\hline \multirow{4}{*}{$\mathrm{L}^{421}$} & $\mathrm{NH}$ & & $\mathrm{S}^{420}$ & $\mathrm{~S}^{420}$ & & & \\
\hline & $\mathrm{C}^{\alpha} \mathrm{H}$ & $\mathrm{Y}^{422}$ & & & & & \\
\hline & $\mathrm{C}^{\beta} \mathrm{H}$ & $\mathrm{Y}^{422}$ & & & & & \\
\hline & $\mathrm{C}^{\gamma} \mathrm{H}$ & $\mathrm{Y}^{422}$ & & & & & \\
\hline $\mathrm{Y}^{422}$ & $\mathrm{NH}$ & & $\mathrm{L}^{421}$ & $\mathrm{~L}^{421}$ & $\mathrm{~L}^{421}$ & & \\
\hline \multirow{2}{*}{$\mathrm{P}^{423}$} & $\mathrm{C}^{\alpha} \mathrm{H}$ & $\mathrm{E}^{424}$ & & & & & \\
\hline & $\mathrm{C}^{\delta} \mathrm{H}$ & $E^{424}$ & & & & & \\
\hline \multirow{3}{*}{$E^{424}$} & $\mathrm{NH}$ & $\mathrm{D}^{425}$ & $\mathrm{P}^{423}$ & & $\mathrm{P}^{423}$ & & \\
\hline & $\mathrm{C}^{\alpha} \mathrm{H}$ & $D^{425}$ & & & & & \\
\hline & $\mathrm{C}^{\beta} \mathrm{H}$ & $D^{425}$ & & & & & \\
\hline \multirow{2}{*}{$D^{425}$} & $\mathrm{NH}$ & $\mathrm{E}^{424}$ & $\mathrm{E}^{424}$ & $\mathrm{E}^{424}$ & & & \\
\hline & $\mathrm{C}^{\alpha} \mathrm{H}$ & $D^{426}$ & & & & & \\
\hline \multirow{2}{*}{$\mathrm{D}^{426}$} & $\mathrm{NH}$ & $L^{427}$ & $\mathrm{D}^{425}$ & & & & \\
\hline & $\mathrm{C}^{\alpha} \mathrm{H}$ & $\mathrm{L}^{427}$ & & & & & \\
\hline $\mathrm{L}^{427}$ & $\mathrm{NH}$ & $\mathrm{D}^{426}$ & $\mathrm{D}^{426}$ & & & & \\
\hline
\end{tabular}




\section{APPENDIX C LIST OF FRAGMENTS FROM PEPTIC DIGESTS OF FXIII(a) and IIa}

Table N: Peak listing of fragments from a peptic digest of human FXIII(a).

\begin{tabular}{|c|c|c|c|}
\hline Residues & Theo. $m / z$ & Sequence & Theo. $\mathrm{D}_{\max }{ }^{a}$ \\
\hline $4-20$ & 1800.92 & SRTAFGGRRAVPPNNSN & 17.1 \\
\hline $19-33$ & 1558.77 & SNAAEDDLPTVELQG & 13.6 \\
\hline $27-36$ & 1038.58 & PTVELQGVVP & 7.4 \\
\hline $31-41$ & 1151.69 & LQGVVPRGVNL & 9.5 \\
\hline $73-90$ & 2212.24 & KLIVRRGQSFYVQIDFSR & 18.2 \\
\hline $75-84$ & 962.55 & IVRRGQSK & 7.5 \\
\hline $88-99$ & 1568.80 & FSRPYDPRRDLF & 11.9 \\
\hline $89-99$ & 1421.73 & SRPYDPRRDLF & 10.9 \\
\hline $98-104$ & 925.51 & LFRVEYV & 6.5 \\
\hline $100-111$ & 1508.79 & RVEYVIGRYPQE & 11.8 \\
\hline $117-132$ & 1680.93 & IPVPIVSELQSGKWGA & 13.5 \\
\hline $125-136$ & 1317.74 & LQSGKWGAKIVM & 11.6 \\
\hline $133-145$ & 1588.90 & KIVMREDRSVRLS & 13.0 \\
\hline $137-144$ & 1030.58 & REDRSVRL & 7.9 \\
\hline $138-156$ & 2101.13 & EDRSVRLSIQSSPKCIVGK & 18.1 \\
\hline $171-180$ & 1176.56 & RTSRNPETDT & 8.9 \\
\hline $178-184$ & 872.44 & TDTYILF & 6.4 \\
\hline $207-218$ & 1339.65 & NDIGVIFYGEVN & 11.5 \\
\hline $220-230$ & 1372.70 & IKTRSWSYGQF & 10.8 \\
\hline $240-247$ & 1013.45 & YVMDRAQM & 7.5 \\
\hline $248-264$ & 1712.94 & DLSGRGNPIKVSRVGSA & 15.9 \\
\hline $298-305$ & 980.54 & ILLEYRSS & 7.5 \\
\hline $328-338$ & 1216.70 & LGIPARIVTNY & 9.5 \\
\hline $364-372$ & 1125.62 & PVPIVSELQSG & 8.4 \\
\hline $380-389$ & 1132.58 & MTRPDLPVGF & 7.5 \\
\hline $380-391$ & 1246.68 & MTRPDLPVGFGG & 9.5 \\
\hline $511-521$ & 1281.59 & VMKSRSNVDMD & 10.7 \\
\hline $513-522$ & 1198.55 & KSRSNVDMDF & 9.7 \\
\hline $526-546$ & 2431.30 & NAVLGKDFKLSITFRNNSHN & 21.2 \\
\hline $535-541$ & 850.48 & LSITFRN & 6.5 \\
\hline $576-592$ & 1844.05 & TLEPLSFKKEAVLIQAG & 15.6 \\
\hline $607-614$ & 949.51 & FVTARINE & 7.5 \\
\hline $607-619$ & 1533.84 & FVTARINETRDVL & 12.8 \\
\hline $617-624$ & 888.52 & DVLAKQKS & 7.5 \\
\hline $632-645$ & 1484.88 & IIIKVRGTQVVGSD & 13.5 \\
\hline $632-646$ & 1615.92 & IIIKVRGTQVVGSDM & 14.7 \\
\hline
\end{tabular}

${ }^{a}$ The maximum number of exchangeable protons within the indicated peptide, assuming $100 \%$ deuteration. This value accounts for all exchangeable backbone amide protons and a slight fraction of N-terminal, C-terminal, and side chain exchangeable protons that are dependent on the final percentage of $\mathrm{D}_{2} \mathrm{O}$ in solution under quench conditions (approximately $4.5 \%$ ). A fully deuterated peptide would theoretically have acquired this amount of deuterons. All peaks were previously identified by Turner and Maurer (174). 
Table O: Peak listing of fragments from a peptic digest of bovine IIa.

\begin{tabular}{ccccc} 
Residues $^{a}$ & Theo. $\mathrm{m} / \mathrm{z}$ & Sequence & Theo. $\mathrm{D}_{\max }{ }^{b}$ & $\mathrm{TCEP}^{c}$ \\
\hline-13 to -4 & 1254.54 & TSEDHFQPFF & 8.5 & $\mathrm{n}$ \\
-3 to 7 & 1924.92 & NEKTFGAGEADCGLRPLF & 16.9 & $\mathrm{y}$ \\
$46-52$ & 888.49 & (L)ISDRNV(L) & 6.6 & $\mathrm{n}$ \\
$65-84$ & 2586.48 & LVRIGKHSRTRYERKVEKISM & 21.8 & $\mathrm{n}$ \\
$85-94$ & 1317.78 & LDKIYIHPRY & 8.8 & $\mathrm{y}$ \\
$85-96$ & 1617.87 & LDKIYIHPRYNW & 10.9 & $\mathrm{y}$ \\
$85-99$ & 2102.12 & LDKIYIHPRYNWKENL & 15.2 & $\mathrm{n}$ \\
$106-113$ & 996.66 & (L)KLKRPIE(L) & 6.7 & $\mathrm{n}$ \\
$106-116$ & 1311.80 & LKLKRPIELSD & 9.8 & $\mathrm{n}$ \\
$135-149 D$ & 2162.12 & KGRVTGWGNRRETWTTSVA & 19.4 & $\mathrm{n}$ \\
$173-180$ & 1018.55 & RIRITDNM & 7.8 & $\mathrm{n}$ \\
$173-181$ & 1165.62 & RIRITDNMF & 8.8 & $\mathrm{n}$ \\
$202-207$ & 1064.53 & KSPYNNRW & 6.8 & $\mathrm{n}$ \\
$212-227$ & 1788.80 & IVSWGEGCDRDGKYGF & 15.9 & $\mathrm{y}$ \\
\hline
\end{tabular}

${ }^{a}$ Residues are numbered according the chymotrypsin numbering scheme described in Chapter I.

${ }^{b}$ The maximum number of exchangeable protons within the indicated peptide, assuming $100 \%$ deuteration. This value accounts for all exchangeable backbone amide protons and a slight fraction of N-terminal, C-terminal, and side chain exchangeable protons that are dependent on the final percentage of $\mathrm{D}_{2} \mathrm{O}$ in solution under quench conditions (approximately $4.5 \%$ ). A fully deuterated peptide would theoretically have acquired this amount of deuterons. ${ }^{c}$ Indicates whether IIa reduction by TCEP is required to observe fragment in mass spectrum. Non-TCEP fragments were identified by Croy et al. (233) and TCEP fragments were identified by both Sabo et al. (167) and Koeppe and Komives (234). 


\section{APPENDIX D ABBREVIATIONS}

(In order of appearance)

Shorthand notation for blood coagulation proteins: FXIII = Factor XIII, etc.

Shorthand notation for activated blood coagulation proteins: FXIIIa=Factor XIIIa, etc

\begin{tabular}{|c|c|}
\hline $\mathrm{TF}$ & Tissue Factor \\
\hline Gla & $\gamma$-carboxyglutamic acid \\
\hline IIa & Thrombin \\
\hline TFPI & Tissue Factor Pathway Inhibitor \\
\hline vWF & von Willebrand Factor \\
\hline PAR & Protease Activated Receptor \\
\hline $\mathrm{HK}$ & High Molecular Weight Kininogen \\
\hline PK & Prekallikrein \\
\hline TAFI & Thrombin Activatable Fibrinolysis Inhibitor \\
\hline $\mathrm{PC}$ & Protein C \\
\hline GAG & Glycosaminoglycan \\
\hline tPA & Tissue-type Plasminogen Activator \\
\hline Gp & Glycoprotein \\
\hline$K_{D}$ & Dissociation Equilibrium Constant \\
\hline $\mathrm{ABE}$ & Anion Binding Exosite \\
\hline LRR & Leucine Rich Repeat \\
\hline Mac-I & Macrophage Antigen-I \\
\hline $\mathrm{FpA}$ & Fibrinopeptide A \\
\hline $\mathrm{Y}_{\mathrm{S}}$ & Sulfotyrosine \\
\hline TGase & Transglutaminase \\
\hline NMR & Nuclear Magnetic Resonance \\
\hline FXIIIa $^{\text {IIa }}$ & Thrombin Activated FXIII \\
\hline $\mathrm{FXIIIa}^{\mathrm{Ca}}$ & Calcium Activated FXIII \\
\hline AT & Antithrombin \\
\hline MALDI & Matrix-Assisted Laser Desorption-Ionization \\
\hline TOF & Time-of-Flight \\
\hline MS & Mass Spectrometry \\
\hline $1 \mathrm{D}$ & One Dimensional \\
\hline $2 \mathrm{D}$ & Two Dimensional \\
\hline $\operatorname{trNOESY}$ & Transferred Nuclear Overhauser Effect Spectroscopy \\
\hline HDX & Hydrogen Deuterium Exchange \\
\hline $\mathrm{DON}$ & 6-diazo-5-oxo-norleucine \\
\hline IAA & Iodoacetamide \\
\hline FID & Free Induction Decay \\
\hline $\mathrm{T}_{1}$ & Spin-lattice Relaxation \\
\hline $\mathrm{T}_{2}$ & Transverse Relaxation \\
\hline$\omega$ & Resonance Frequency \\
\hline$\tau_{\mathrm{c}}$ & Rotational Correlation Time \\
\hline $\mathrm{k}_{\text {off }}$ & Dissociation Rate Constant \\
\hline TOCSY & Total Correlation Spectroscopy \\
\hline
\end{tabular}




\begin{tabular}{|c|c|}
\hline $\mathrm{DE}$ & Delayed Extraction \\
\hline$m / z$ & Mass to Charge ratio \\
\hline$\alpha-\mathrm{CHCA}$ & $\alpha$-cyano-4-hydroxycinnamic acid \\
\hline NEM & N-ethylmaleimide \\
\hline $\mathrm{AA}$ & Acetic Anhydride \\
\hline $\mathrm{k}_{\mathrm{u}}$ & Unfolding Rate Constant \\
\hline $\mathrm{k}_{\mathrm{f}}$ & Folding Rate Constant \\
\hline $\mathrm{k}_{\mathrm{op}}$ & Local Unfolding Rate Constant \\
\hline $\mathrm{k}_{\mathrm{cl}}$ & Local Folding Rate Constant \\
\hline $\mathrm{k}_{\mathrm{obs}}$ & Observed Rate Constant \\
\hline TCEP & Tris (2-carboxyethyl) phosphine \\
\hline PSD & Post Source Decay \\
\hline$Y_{P}$ & Phosphotyrosine \\
\hline BSE & Barium sulfate eluate \\
\hline EDTA & Ethylenediaminetetraacetate \\
\hline MWCO & Molecular Weight Cut-Off \\
\hline PPACK & D-Phe-Arg-Pro-chloromethyl ketone \\
\hline TFA & Trifluoroacetic acid \\
\hline AUC & Analytical Ultracentrifugation \\
\hline $\mathrm{K}_{\mathrm{I}}$ & Inhibition Equilibrium Constant \\
\hline $\mathrm{IC}_{50}$ & Concentration Resulting in $50 \%$ Inhibition \\
\hline SV & Sedimentation Velocity \\
\hline $\mathrm{S}$ & Svedberg Constant \\
\hline DMSO & Dimethylsulfoxide \\
\hline GDH & Glutamate Dehydrogenase \\
\hline GEE & Glycine ethyl ester \\
\hline $\mathrm{NADH}$ & Nicotinamide adenine dinucleotide \\
\hline FXIII(a) & Unactivated, Activated and Inhibited Factor XIII \\
\hline $\mathrm{DMF}$ & Dimethyl formamide \\
\hline$\alpha_{2} \mathrm{AP}$ & $\alpha_{2}$-antiplasmin \\
\hline $\mathrm{AP}$ & Activation Peptide \\
\hline ITC & Isothermal Calorimetry \\
\hline$\Delta \mathrm{H}$ & Enthalpy \\
\hline$\Delta \mathrm{G}$ & Gibbs Free Energy \\
\hline$\Delta \mathrm{S}$ & Entropy \\
\hline $\mathrm{R}$ & Gas Constant \\
\hline $\mathrm{T}$ & Absolute Temperature \\
\hline HSQC & Heteronuclear Single Quantum Coherence \\
\hline PFG & Pulsed Field-Gradient \\
\hline
\end{tabular}




\section{CURRICULUM VITAE}

\section{Thomas Michael Sabo, II}

Department of Chemistry

University of Louisville

Louisville, KY 40292

(502) 852-3578

e-mail: mike.sabo@louisville.edu

\section{EDUCATION}

B.S. Chemistry with a concentration in Biochemistry, May 2002, University of Louisville, Louisville, KY

M.S. Chemistry, December 2006, University of Louisville, Louisville, KY

Ph.D. Chemistry, December 2007, University of Louisville, Louisville, KY

(Advisor: Dr. Muriel C. Maurer)

\section{AWARDS AND HONORS}

- Graduated in May 2002 with Magna Cum Laude honors

- American Chemical Society Award in Chemistry 2002, Award for the year's outstanding graduate in chemistry

- Chemistry Research Scholars Fellowship 2003-2004

- IMD3 Travel Award 2004 and 2007

\section{RESEARCH EXPERIENCE}

\section{Graduate Research}

Department of Chemistry, University of Louisville, Louisville, KY (2003-present) Advisor: Dr. Muriel C. Maurer

- Research focus is centered on the serine protease thrombin and the transglutaminase Factor XIII, two enzymes involved in the latter stages of blood coagulation.

- Activation and purification of bovine thrombin from bovine sulfate eluate employing HPLC, size exclusion and cation exchange chromatography, SDSPAGE, and MALDI-TOF MS 
- Enhancement of the Hydrogen/Deuterium Exchange coupled with MALDI-TOF MS protocol through addition of the reducing agent TCEP which is effective at the acidic $\mathrm{pH}$ quench conditions

- Characterization of conformational features associated with the $\gamma^{\prime}$-peptide (410427) from fibrin(ogen) and a C-terminal fragment of GpIbo (269-286) binding to thrombin's anion binding exosite II

○ $1 \mathrm{D}{ }^{1} \mathrm{H}$ and ${ }^{31} \mathrm{P}$ line broadening NMR, 2D TOCSY ${ }^{1} \mathrm{H}$ NMR, and 2D transferred NOESY ${ }^{1} \mathrm{H}$ NMR methods were used to identify key peptide residues involved in binding to ABE-II and some of the secondary structural features adopted by the peptides upon interaction with thrombin

- HDX coupled with MALDI-TOF MS was implemented to determine where within thrombin's ABE-II these peptides are interacting, as well as any long range, allosteric consequences to thrombin resulting from peptide binding to ABE-II

- Isothermal calorimetry and analytical ultracentrifugation were applied to thermodynamically describe the interaction

- Investigation into the efficacy of a novel peptide inhibitor of FXIII using a coupled uv-vis assay

- Studied the conformational consequences of activation and inhibition of FXIII with MALDI-TOF MS utilizing:

$\circ$ chemical modification of cysteine and lysine residues

- limited proteolysis with chymotrypsin and trypsin

$\circ$ hydrogen/deuterium exchange

\section{Undergraduate Research}

Department of Pediatric Nephrology, University of Georgetown Medical Center, Washington, DC (June-August 2001) Advisor: Dr. Pedro A. Jose

- The focus of the research centered on understanding the molecular mechanisms of hypertension through techniques in molecular biology

- Contributed to testing the hypothesis that the increase in urinary sodium excretion following feeding was due to excess gastrin inhibiting tubular $\mathrm{Na}^{+} / \mathrm{K}^{+}$ATPase activity

- Implemented antisense oligonucleotides to study the consequences of ligand binding to the dopamine receptors D1 and D5

- Utilized human cell cultures, transgenic mice, $\mathrm{Na}^{+} / \mathrm{K}^{+}$ATPase kinetic assays, and western blotting

Department of Chemistry, University of Louisville, Louisville, KY (May 2000-April 2002) Advisor: Dr. K.G. Taylor

- Organic Synthesis

- Synthesized derivatives of mannose for eventual linkage to proteins.

- Synthesized thiol based compounds for flavor enhancement of grape juice. 


\section{TEACHING EXPERIENCE}

Teaching Assistant, Chemistry, University of Louisville (Fall 2002-Spring 2004)

- Biochemistry Laboratory (Chem 546)

- Organic Chemistry Recitation (Chem 342)

- Honors Organic Chemistry Laboratory (Chem 343)

- Organic Chemistry Laboratory (Chem 343)

\section{PUBLICATIONS}

- Sabo, T. M., Brasher, P. B., and Maurer, M. C. (2007) Perturbations in Factor XIII Resulting from Activation and Inhibition Examined by Solution Based Methods and Detected by MALDI-TOF MS, Biochemistry. 46, 10089 - 10101.

- Sabo, T. M., Farrell, D. H., and Maurer, M. C. (2006) Conformational analysis of $\gamma^{\prime}$ peptide (410-427) interactions with thrombin anion binding exosite II. Biochemistry $45,7434-45$.

- Turner, B. T., Jr., Sabo, T. M., Wilding, D., and Maurer, M. C. (2004) Mapping of factor XIII solvent accessibility as a function of activation state using chemical modification methods. Biochemistry 43, 9755-65.

\section{ORAL PRESENTATION}

- Sabo, T. M. and Maurer, M. C. "Biophysical Analysis of GpIba (269-286) Peptide Interactions with Thrombin's Anion Binding Exosite II." FASEB Summer Research Conference: Proteases in Hemostasis and Vascular Biology, Indian Wells, CA. June 2-7 2007

\section{POSTER ABSTRACTS}

- Sabo, T. M. and Maurer, M. C. "Biophysical Analysis of GpIba (269-286) Peptide Interactions with Thrombin's Anion Binding Exosite II." Twenty-First Annual Symposium of the Protein Society, Boston, MA. July 21-25, 2007

- Sabo, T. M., Brasher, P. B., and Maurer, M. C. "Differences in Solvent Accessibility that Occur to FXIIIa in the Presence of Active Site Directed Ligands." Twentieth Annual Symposium of the Protein Society, San Diego, CA. August 5-9, 2006

- Sabo, T. M. and Maurer, M. C. "Evaluating the Binding of the $\gamma^{\prime}$ Peptide (410-427) and the Effects of $[\mathrm{NaCl}]$ on Thrombin Solvent Accessibility Using HDX MALDITOF MS." Nineteenth Annual Symposium of the Protein Society, Boston, MA. July 30-August 3, 2005

- Sabo, T. M., Farrell, D. H., and Maurer, M. C. "Characterizing the Binding of Fibrinogen $\gamma^{\prime}$ (410-427) to Thrombin Anion Binding Exosite II by Solution NMR Methods" Eighteenth Annual Symposium of the Protein Society, San Diego, CA. August 14-18, 2004 
- $\quad$ Turner, B. T., Jr., Sabo, T. M., Wilding, D. and Maurer, M. C. "Mapping the Solvent Accessibility of Factor XIII as a Function of Activation State Using Chemical Modification Methods" Eighteenth Annual Symposium of the Protein Society, San Diego, CA. August 14-18, 2004

\section{PROFESSIONAL ACTIVITIES}

- Treasurer, Chemistry Graduate Student Association, 2003-2004

- Poster Judge, Kentuckiana Undergraduate Research Symposium, 2004

\section{PROFESSIONAL SOCIETIES}

- American Chemical Society 2004-

- Protein Society 2004-

\section{OTHER INTERESTS}

- Guitarist/Pianist with two records

- J Kessinger and the Verlaines, La Musique de Jacques Brel, 2003 (Debauchery)

- Helgeson Story, Stories, 1999 (Landmark Recordings)

- Currently a member of Follow the Train (Darla) 